WSRC-TR-2000-00347

\title{
Electromagnetic Borehole Flowmeter (EBF) Testing at the Southwest Plume Test Pad (U)
}

Westinghouse Savannah River Company Savannah River Site Aiken, SC 29808

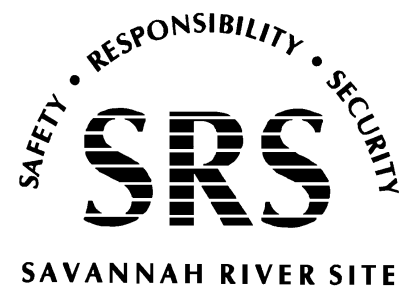


This document was prepared in conjunction with work accomplished under Contract No.

DE-AC09-96SR18500 with the U.S. Department of Energy.

\section{DISCLAIMER}

This report was prepared as an account of work sponsored by an agency of the United States Government. Neither the United States Government nor any agency thereof, nor any of their employees, makes any warranty, express or implied, or assumes any legal liability or responsibility for the accuracy, completeness, or usefulness of any information, apparatus, product or process disclosed, or represents that its use would not infringe privately owned rights. Reference herein to any specific commercial product, process or service by trade name, trademark, manufacturer, or otherwise does not necessarily constitute or imply its endorsement, recommendation, or favoring by the United States Government or any agency

thereof. The views and opinions of authors expressed herein do not necessarily state or reflect those of the United States Government or any agency thereof.

This report has been reproduced directly from the best available copy.

Available for sale to the public, in paper, from: U.S. Department of Commerce, National Technical Information Service, 5285 Port Royal Road, Springfield, VA 22161, phone: (800)

553-6847, fax: (703) 605-6900, email: orders@ntis.fedworld.gov online ordering: http://www.ntis.gov/ordering.htm

Available electronically at http://www.doe.gov/bridge

Available for a processing fee to U.S. Department of Energy and its contractors, in paper, from: U.S. Department of Energy, Office of Scientific and Technical Information, P.O. Box 62, Oak Ridge, TN 37831-0062, phone: (865 ) 576-8401, fax: (865) 576-5728, email: reports@ adonis.osti.gov 
WSRC-TR-2000-00347

Publication Date: September 2000

\section{Electromagnetic Borehole Flowmeter (EBF) Testing at the Southwest Plume Test Pad (U)}

Gregory P. Flach, Frank C. Sappington, Fatina A. Washburn and Robert A. Hiergesell

Westinghouse Savannah River Company Savannah River Site Aiken, SC 29808

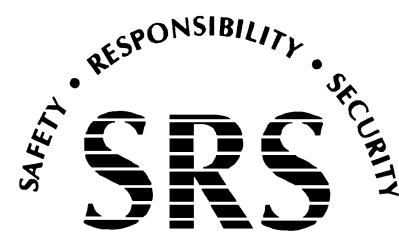


ii

WSRC-TR-2000-00347, Rev. 0, Electromagnetic Borehole Flowmeter Testing at the SPTP (U)

Electromagnetic Borehole Flowmeter Testing at the Southwest Plume Test Pad (U)

Authentication and Approvals:
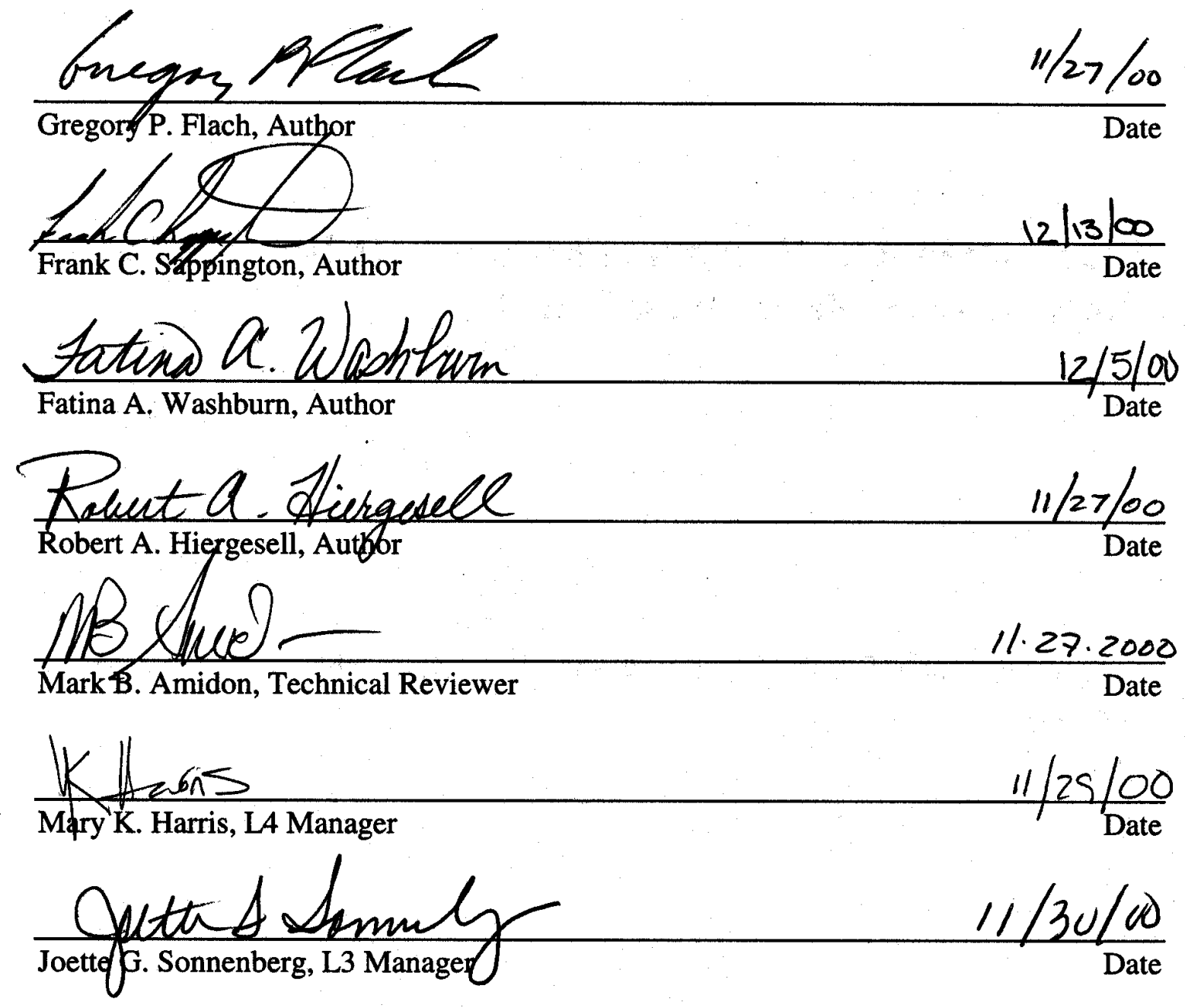


\section{EXECUTIVE SUMMARY}

Multiple-well aquifer tests were recently conducted at the Southwest Plume Test Pad near the Burial Ground Complex (BGC) in accordance with the BGC Field Investigation Plan (WSRC, 1999). The pumping tests were performed in the Upper Three Runs and Gordon aquifers in February and March of 1999. The tests provide reliable estimates of horizontal conductivity averaged over aquifer thickness, and a relatively large horizontal zone of influence. Table 1 summarizes the results of WSRC (1999). To complement these results, Electromagnetic Borehole Flowmeter (EBF) testing using the 1" ID EBF was performed to determine the vertical variation of horizontal conductivity for SWP-300A, SWP-200C and SWP-100D.

EBF testing indicates significant heterogeneity over the tested screen zones (Figures 8, 10 and 12). At SWP-300A in the Gordon aquifer, two high conductivity zones separated by a low conductivity interval are observed in the lower half of the screen. Conductivity is relatively low in the upper half of the screen. At SWP-200C in the lower Upper Three Runs Aquifer (UTRA), two high conductivity zones separated by a low conductivity interval are observed in the middle of the screen zone. At SWP-100D in the upper UTRA, conductivity generally increases from top to bottom of the screen.

Regarding future deployments of the technology, experience at the Southwest Plume Test Pad (SPTP) confirms that the 1" ID EBF is preferred over the 1/2" ID EBF for dynamic testing. The 1" EBF has more than adequate sensitivity and practically eliminates bypass flow through the filter pack. Testing at the SPTP also revealed that the EBF packer cannot achieve a good seal against a wire wrap screen, which leads to flow bypassing the flowmeter between the casing and packer bellows. The raw EBF flow data were corrected for internal bypass flow by comparing the flow measured at screen joints, where a good seal was achieved, to adjacent flows. Other considerations being equal, slotted screen is preferred over wire wrap screen because a good seal can be achieved and bypass flow corrections avoided. Equipment failures and design deficiencies with the current EBF system continue to be a problem. A more reliable EBF system is needed for future deployments to be efficient.

Single-well aquifer testing performed concurrently with EBF testing was investigated as an possible alternative to reliance on prior multiple-well estimates of average horizontal conductivity or well efficiency. Single-well testing refers to conventional estimates of screen-average conductivity and estimates of subinterval conductivity using CooperJacob analysis. As with all single-well testing, either approach depends on an uncertain estimate of well efficiency in the absence of prior multiple-well testing. Concurrent single-well aquifer testing was determined to be feasible in the field. Based on EBF testing in R-area and at the Southwest Plume Test Pad, conductivity estimates based on single-well testing alone have an uncertainty of roughly plus or minus a factor of 2 . A prior multiple-well estimate of average conductivity is technically preferred, but concurrent single-well testing may be preferred overall considering the cost of multiplewell aquifer testing. 
This page intentionally left blank 


\section{Contents}

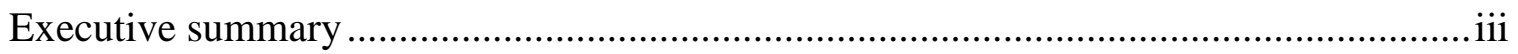

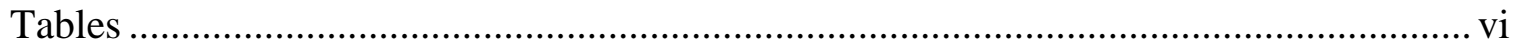

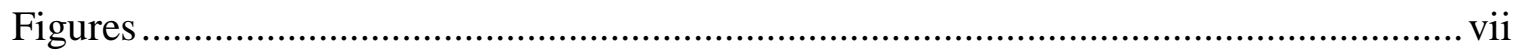

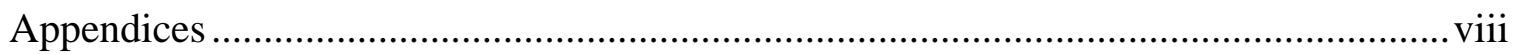

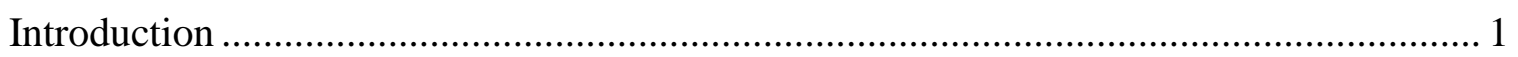

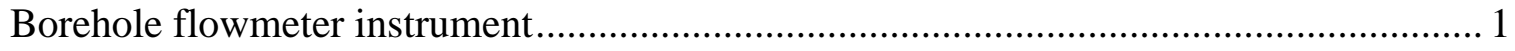

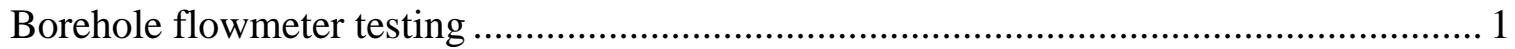

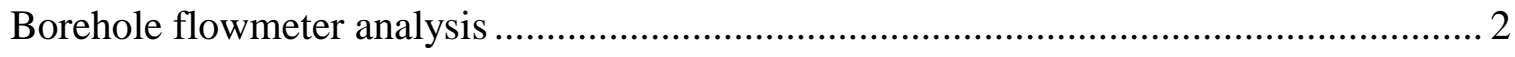

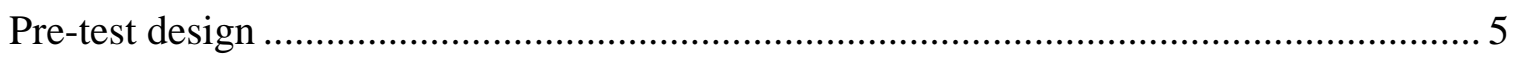

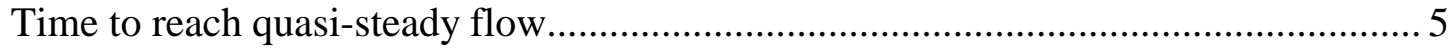

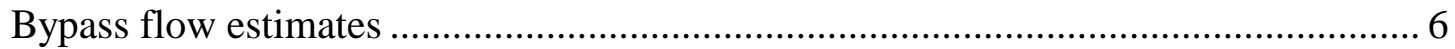

Field experience at the Southwest Plume Test Pad ..................................................... 7

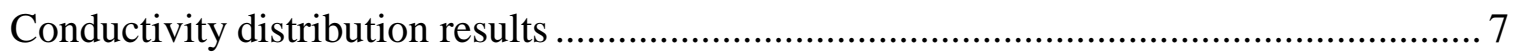

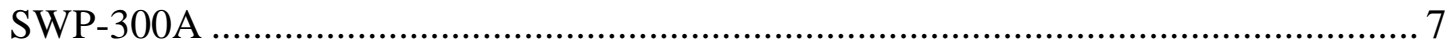

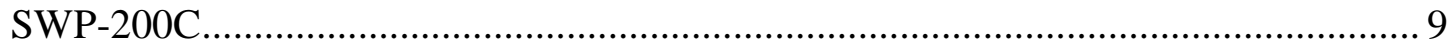

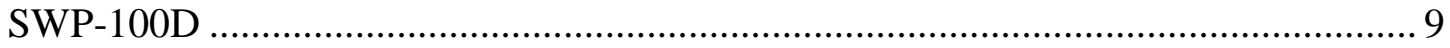

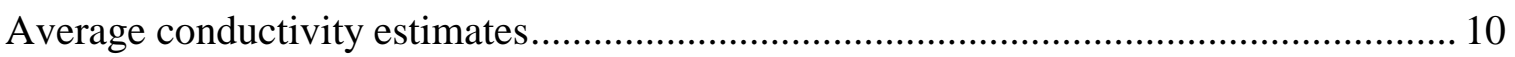

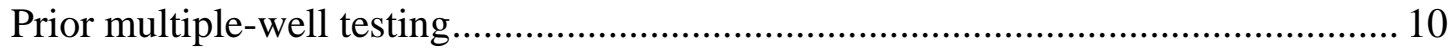

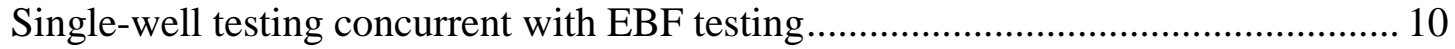

Alternative conductivity estimates using Cooper-Jacob analysis.................................. 11

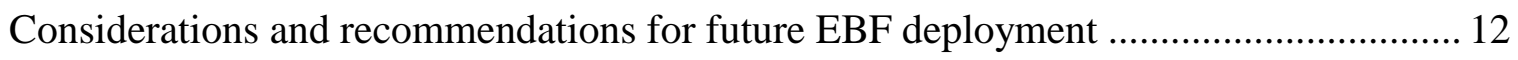

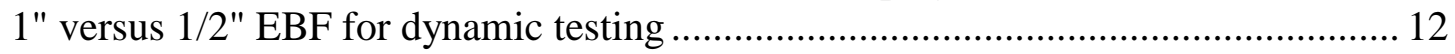

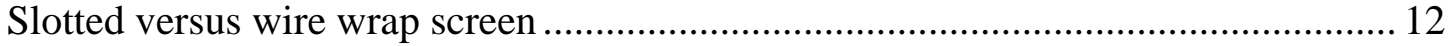

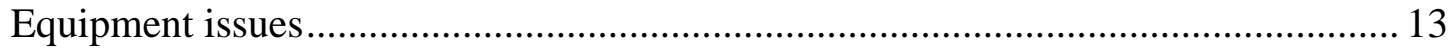

Feasibility of concurrent single-well aquifer testing ........................................... 13

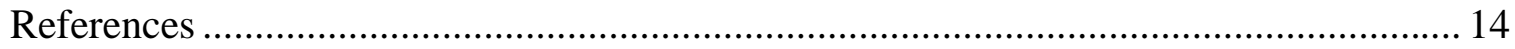




\section{Tables}

Table $1 \quad$ SPTP well information ................................................................ 16

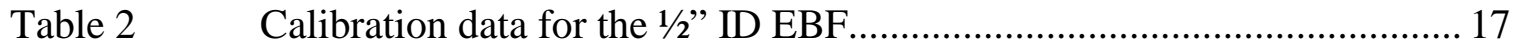

Table 3 Calibration data for the 1" ID EBF....................................................... 18

Table 4 Pre-test design calculations for SWP-300A ........................................ 19

Table 5 Pre-test design calculations for SWP-200C ......................................... 20

Table 6 Pre-test design calculations for SWP-100D ......................................... 21

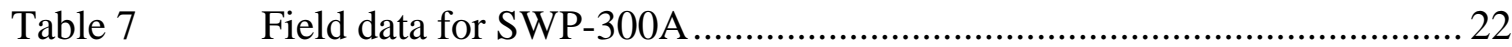

Table $8 \quad$ Hydraulic conductivity calculations for SWP-300A at $1 \mathrm{ft}$

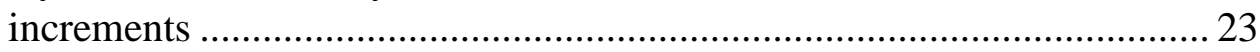

Table 9 Hydraulic conductivity calculations for SWP-300A at $2 \mathrm{ft}$ increments ..................................................................................... 24

Table $10 \quad$ Hydraulic conductivity calculations for SWP-300A at $5 \mathrm{ft}$

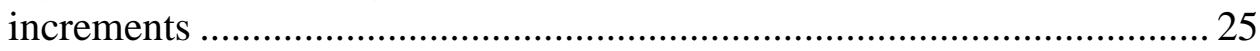

Table 11 Hydraulic conductivity calculations for SWP-300A at $10 \mathrm{ft}$

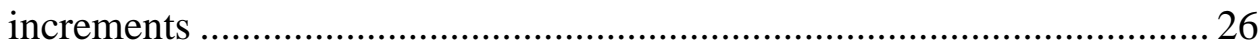

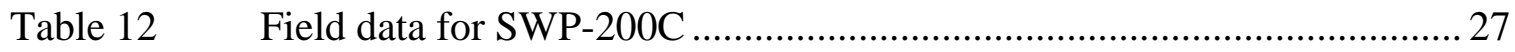

Table 13 Hydraulic conductivity calculations for SWP-200C at $2 \mathrm{ft}$

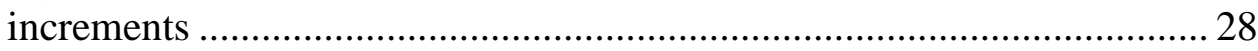

Table 14 Hydraulic conductivity calculations for SWP-200C at $6 \mathrm{ft}$

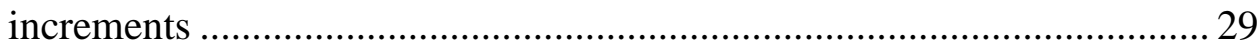

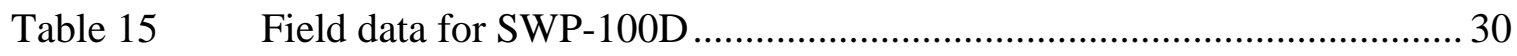

Table 16 Hydraulic conductivity calculations for SWP-100D at $1 \mathrm{ft}$

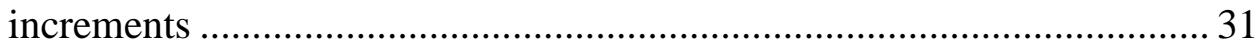

Table 17 Hydraulic conductivity calculations for SWP-100D at $3 \mathrm{ft}$

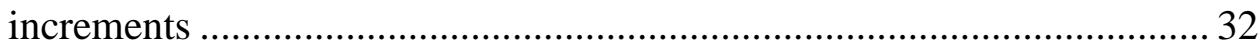

Table 18 Hydraulic conductivity calculations for SWP-100D at $7 \mathrm{ft}$

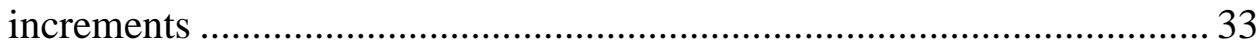

Table 19 Comparison of average conductivity estimates .................................... 34 


\section{Figures}

Figure 1 Schematic diagram of the Electromagnetic Borehole Flowmeter; reproduced from Molz and Young (1993) 35

Figure 2 Electromagnetic Borehole Flowmeter (EBF) application of Faraday's Law of Induction; reproduced from Molz and Young (1993) 36

Figure 3 Calibration data and curve for the $1 / 2$ " ID EBF 37

Figure 4 Calibration data and curve for the 1" ID EBF. 37

Figure 5 Schematic illustration of borehole flowmeter testing; reproduced from Molz and Young (1993)

Figure 6 Basic geometry and analysis of borehole flowmeter data; reproduced from Molz and Young (1993) .... 39

Figure 7 Ambient flow measurements for SWP-300A.

Figure 8 Estimated relative hydraulic conductivity profile for SWP-300A at $1,2,5$ and $10 \mathrm{ft}$ resolutions 40

Figure 9 Ambient flow measurements for SWP-200C

Figure 10 Estimated relative hydraulic conductivity profile for SWP-200C at 2 and $6 \mathrm{ft}$ resolutions 41

Figure 11 Ambient flow measurements for SWP-100D. 42

Figure 12 Estimated relative hydraulic conductivity profile for SWP-100D at 1,3 and $7 \mathrm{ft}$ resolutions

Figure 13 Comparison of EBF relative conductivity profiles to natural gamma $\log$.....

Figure 14 Time-drawdown data and single-well conductivity estimates for SWP-300A

Figure 15 Time-drawdown data and single-well conductivity estimates for SWP-200C.

Figure 16 Time-drawdown data and single-well conductivity estimates for SWP-100D 46

Figure 17 Unaltered and smoothed water level transducer data for SWP-300A....... 47

Figure 18 Unaltered and smoothed water level transducer data for SWP-200C ....... 47

Figure 19 Unaltered and smoothed water level transducer data for SWP-100D ....... 48 


\section{Appendices}

Appendix A Time-drawdown data for SWP-300A …......................................... A-1

Appendix B Time-drawdown data for SWP-200C ................................................... B-1

Appendix C Time-drawdown data for SWP-100D …............................................. C-1 


\section{Introduction}

Multiple-well aquifer tests were recently conducted at the Southwest Plume Test Pad (SPTP) near the Burial Ground Complex (BGC) in accordance with the BGC Field Investigation Plan (WSRC, 1999). The pumping tests were performed in the Upper Three Runs and Gordon aquifers in February and March of 1999. The tests provide reliable estimates of horizontal conductivity averaged over aquifer thickness, and a relatively large horizontal zone of influence. Table 1 summarizes the results of WSRC (1999). To complement these results, Electromagnetic Borehole Flowmeter (EBF) testing was performed to determine the vertical variation of horizontal conductivity about the average determined from the conventional aquifer testing. This report introduces the borehole flowmeter concept and EBF instrument, summarizes field and calculation procedures, presents EBF test results for 3 SPTP wells, and provides recommendations for future application of the technology.

\section{Borehole flowmeter instrument}

The term "borehole flowmeter" in this report refers to any instrument that measures the vertical flow inside a well casing. Various types of borehole flowmeters have been used in field applications, including heat pulse, tracer release and impeller (spinner) designs. Researchers at the Tennessee Valley Authority (TVA) developed, patented and commercialized a robust, highly-sensitive, borehole flowmeter based on electromagnetic principles. The Electromagnetic Borehole Flowmeter operates according to Faraday's Law of Induction, which states that the voltage induced by a conductor moving at right angles through a magnetic field is directly proportional to the velocity of the moving conductor (Waldrop, 1995). Schematic diagrams of the EBF are shown in Figures 1 and 2 (Molz and Young, 1993). Groundwater acts as the moving conductor, an electromagnet generates the magnetic field, and electrodes measure the induced voltage. The $1 / 2$ " ID $\mathrm{EBF}$ has a threshold flowrate of about $5 \mathrm{~mL} / \mathrm{min}$, which is 1000 times more sensitive than the typical impeller flowmeter. Flowrates up about $10 \mathrm{~L} / \mathrm{min}$ can be measured, giving the $1 / 2$ " ID EBF outstanding range. The 1" ID EBF can measure flows from about $40 \mathrm{~mL} / \mathrm{min}$ to $40 \mathrm{~L} / \mathrm{min}$ (Waldrop, 1995). Both the $1 / 2$ " and 1 " EBF instruments were used for aquifer testing at the SPTP site. Calibration data for both EBF instruments are provided in Tables 2 and 3 and Figures 3 and 4. The calibration curves are developed in Flach and others (2000).

\section{Borehole flowmeter testing}

The idea behind borehole flowmeter testing is to relate horizontal conductivity as a function of elevation, $K(z)$, to borehole discharge as a function of elevation $Q(z)$. The field procedure is schematically illustrated in Figure 5 (Molz and Young, 1993). Under quasi-steady pumping conditions, borehole discharge $(\mathrm{Q})$ from the bottom of the screen up to the current flowmeter position is measured as a function of elevation (z). As shown in Figure 6, the difference $(\Delta Q)$ in borehole discharge $Q(z)$ between at any two locations is the flowrate of groundwater entering the well casing over that interval. This differential flowrate, minus any ambient flow effects, is proportional to the horizontal 
conductivity of the aquifer over that interval. Ambient flow refers to horizontal flow through the well screen and vertical flow in the casing under natural, undisturbed conditions. In order to rigorously account for potential ambient flow effects, the standard borehole flowmeter test procedure actually involves two series of measurements:

1) under ambient conditions, measure the vertical flowrate inside the well screen at 1 to $2 \mathrm{ft}$ intervals,

2) pump (or inject) at a constant rate above the screen zone and borehole flowmeter,

3) pause until the drawdown becomes quasi-steady-state,

4) under these quasi-steady-state pumping conditions, again measure the vertical flowrate inside the well screen at 1 to $2 \mathrm{ft}$ intervals.

The quasi-steady conditions referred to in step 3) typically occur within 15 to 30 minutes in confined aquifers, and a couple of hours in unconfined aquifers (Flach and others, 2000). The ambient flow data is also useful by itself for determining the direction(s) of vertical head gradients in the surrounding aquifer, which has contaminant monitoring implications discussed by Flach and others (2000).

\section{Borehole flowmeter analysis}

Molz and others (1989) and Molz and Young (1993) present two methods for estimating the vertical variation of horizontal conductivity from field measurements of borehole discharge as a function of depth, such as those provided by the EBF (see also Rehfeldt and others (1989)). Both methods assume a fully-penetrating well in a confined aquifer. The first method applies the Cooper-Jacob (1946) method to individual sub-intervals of the well screen to estimate conductivity as a function of vertical position. Alternatively, the relative variation of horizontal conductivity about vertically-averaged $\mathrm{K}$ can be directly related to the EBF measurements of borehole discharge as a function of depth. If the vertical average is known or can be estimated by other means (e.g. pumping test), then the conductivity profile can be determined. The first method produces conductivity estimates from EBF test data alone, but involves a number of uncertain parameters. The second method is simpler and more precise, but requires an independent estimate of average conductivity. At the Southwest Plume Test Pad, a conventional pumping test result is available for each EBF tested well. Therefore, the second approach presented by Molz and Young (1993) is preferred for the present application. Nevertheless, both methods are presented in order to later compare methods.

As discussed previously, the field procedure involves measuring the vertical flowrate within the well casing at regular intervals, typically every 1 to $2 \mathrm{ft}$, first under ambient conditions and then under pumping conditions. The difference between any two readings is the flow entering the well between the two corresponding elevations. A negative value implies that flow is leaving the well. To develop the first method, the Cooper-Jacob equation is applied to each layer as 


$$
\Delta \mathrm{h}_{\mathrm{i}}\left(\mathrm{r}_{\mathrm{w}}, \mathrm{t}\right)=\frac{\left(\Delta \mathrm{Q}_{\mathrm{i}}-\Delta \mathrm{q}_{\mathrm{i}}\right)}{2 \pi \mathrm{K}_{\mathrm{i}} \Delta \mathrm{z}_{\mathrm{i}}} \ln \left(\frac{1.5}{\mathrm{r}_{\mathrm{w}}} \sqrt{\frac{\mathrm{K}_{\mathrm{i}} \Delta \mathrm{z}_{\mathrm{i}} \mathrm{t}}{\mathrm{S}_{\mathrm{si}} \Delta \mathrm{z}_{\mathrm{i}}}}\right)
$$

where

$$
\begin{aligned}
& \Delta \mathrm{h}_{\mathrm{i}} \equiv \text { drawdown } \\
& \Delta \mathrm{Q}_{\mathrm{i}}-\Delta \mathrm{q}_{\mathrm{i}} \equiv \text { net differential flow } \\
& \Delta \mathrm{Q}_{\mathrm{i}} \equiv \text { difference in EBF flow at the top and bottom of the } \mathrm{i}^{\text {th }} \text { interval under } \\
& \text { pumping conditions } \\
& \Delta \mathrm{q}_{\mathrm{i}} \equiv \text { difference in EBF flow at the top and bottom of the } \mathrm{i}^{\text {th }} \text { interval under } \\
& \text { ambient conditions } \\
& \mathrm{K}_{\mathrm{i}} \equiv \text { horizontal conductivity of } \mathrm{i}^{\mathrm{th}} \text { layer } \\
& \Delta \mathrm{z}_{\mathrm{i}} \equiv \text { thickness of } \mathrm{i}^{\text {th }} \text { layer } \\
& \mathrm{r}_{\mathrm{w}} \equiv \text { well bore radius } \\
& \mathrm{t} \equiv \text { elapsed time } \\
& \mathrm{S}_{\mathrm{si}} \equiv \text { specific storage of } \mathrm{i}^{\text {th }} \text { layer }
\end{aligned}
$$

Assuming negligible borehole flowmeter losses under low flow conditions, each layer experiences the same measured drawdown, $\Delta \mathrm{h}$. The numerical simulations of Javendel and Witherspoon (1969) indicate that the transient drawdown responses of layers in a heterogeneous aquifer quickly merge into a common response described by the Theis solution using averaged aquifer properties. This observation suggests that each layer effectively behaves as if it has the hydraulic diffusivity of the entire aquifer:

$$
\frac{\mathrm{K}_{\mathrm{i}} \Delta \mathrm{z}_{\mathrm{i}}}{\mathrm{S}_{\mathrm{si}} \Delta \mathrm{z}_{\mathrm{i}}}=\frac{\mathrm{T}}{\mathrm{S}}=\mathrm{v}
$$

In this expression

$$
\begin{aligned}
& v \equiv \text { hydraulic diffusivity of the entire aquifer } \\
& T \equiv \text { transmissivity of the entire aquifer } \\
& \mathrm{S} \equiv \text { storage coefficient (storativity) of the entire aquifer }
\end{aligned}
$$

With this assumption, one of two proposed by Molz and others (1989), equation (1) becomes after solving for $\mathrm{K}_{\mathrm{i}}$ 


$$
\mathrm{K}_{\mathrm{i}}=\frac{\left(\Delta \mathrm{Q}_{\mathrm{i}}-\Delta \mathrm{q}_{\mathrm{i}}\right)}{2 \pi \Delta \mathrm{z}_{\mathrm{i}} \Delta \mathrm{h}} \ln \left(\frac{1.5}{\mathrm{r}_{\mathrm{w}}} \sqrt{\frac{\mathrm{Tt}}{\mathrm{S}}}\right)
$$

With a prior estimate of aquifer diffusivity (T/S), equation (3) provides a direct estimate of horizontal conductivity for the $i^{\text {th }}$ layer. Fortunately, the Cooper-Jacob estimate is not highly sensitive to the assumed value of T/S for large times, because diffusivity appears within a square-root and logarithm. However, well inefficiencies introduce significant uncertainty in the drawdown experienced by the formation, $\Delta \mathrm{h}$.

The data analysis procedure for the second method presented by Molz and Young (1993) is summarized by

$$
\frac{\mathrm{K}_{\mathrm{i}}}{\overline{\mathrm{K}}}=\frac{\left(\Delta \mathrm{Q}_{\mathrm{i}}-\Delta \mathrm{q}_{\mathrm{i}}\right) / \Delta \mathrm{z}_{\mathrm{i}}}{\sum_{\mathrm{i}}\left(\Delta \mathrm{Q}_{\mathrm{i}}-\Delta \mathrm{q}_{\mathrm{i}}\right) / \sum_{\mathrm{i}} \Delta \mathrm{z}_{\mathrm{i}}}
$$

where

$$
\begin{aligned}
\mathrm{K}_{\mathrm{i}} \equiv & \text { horizontal conductivity of the } \mathrm{i}^{\text {th }} \text { interval } \\
\overline{\mathrm{K}} \equiv & \text { vertically-averaged conductivity } \\
\Delta \mathrm{Q}_{\mathrm{i}} \equiv & \begin{array}{l}
\text { difference in EBF flow at the top and bottom of the } \mathrm{i}^{\text {th }} \text { interval under } \\
\quad \text { pumping conditions }
\end{array} \\
\Delta \mathrm{q}_{\mathrm{i}} \equiv & \begin{array}{l}
\text { difference in EBF flow at the top and bottom of the } \mathrm{i}^{\text {th }} \text { interval under } \\
\text { ambient conditions }
\end{array} \\
\Delta \mathrm{z}_{\mathrm{i}} \equiv & \text { height of the } \mathrm{i}^{\text {th }} \text { interval. }
\end{aligned}
$$

In equation (4), $\left(\Delta \mathrm{Q}_{\mathrm{i}}-\Delta \mathrm{q}_{\mathrm{i}}\right)$ is the net flowrate induced by pumping and accounts for ambient flow effects. Note that the relative conductivity distribution is equal to the relative distribution of net flow entering the well, which is assumed to occur after the initial transient passes and quasi-steady conditions develop. The basis for this assumption is considered in detail by Flach and others (2000).

Both methods, summarized by equations (3) and (4), assume that flow approaching the well is horizontal and driven by a vertically-uniform radial head gradient. Any deviations from this assumption in the flow field will introduce systematic biases in the relative conductivity profile computed from either equation. Therefore, pre-testing consideration should be given to identifying, and minimizing if possible, any conditions that will violate the above assumption. For example, non-horizontal flow will occur in an unconfined aquifer if the drawdown is significant, but can be minimized by pumping at a small rate. A partially-penetrating well screen would also create vertical flows. More subtle sources of vertical flow leading to systematic errors are 1) transient effects in the 
presence of aquifer heterogeneity, 2) a high conductivity filter pack, and 3) head losses across the EBF. Flach and others (2000) further discuss each type of systematic error.

\section{Pre-test design}

Pre-test design calculations were performed to ensure quasi-steady flow conditions would be achieved before EBF testing started, and to minimize systematic errors related to head losses across the EBF, such as bypass flow.

Time to reach quasi-steady flow: Conductivity profiles estimated from equations (3) or (4) depend on the flow having reached quasi-steady conditions. Four possible criteria for defining the elapsed time required after pumping to achieve quasi-steady conditions have been identified by Flach and others (2000, eqns. (10), (13), (14) and (15)). They are summarized by

$$
\begin{gathered}
\mathrm{t}_{\mathrm{D}}=\frac{4 \mathrm{Tt}}{\mathrm{Sr}_{\mathrm{w}}^{2}}=\frac{4 \mathrm{vt}}{\mathrm{r}_{\mathrm{w}}^{2}}>4 \times 10^{2} \text { to } 4 \times 10^{3} \\
\mathrm{t}_{\mathrm{D}}>1000 \frac{1+\mathrm{v}_{2} / \mathrm{v}_{1}}{2} \\
\mathrm{t}_{\mathrm{D}}>10^{2}\left(\frac{\mathrm{r}_{\mathrm{i}}}{\mathrm{r}_{\mathrm{w}}}\right)^{2} \\
\mathrm{t}_{\mathrm{D}}>10^{6}
\end{gathered}
$$

where

$$
\begin{aligned}
& \mathrm{t} \equiv \text { elapsed time } \\
& \mathrm{T} \equiv \text { transmissivity of the entire aquifer } \\
& \mathrm{S} \equiv \text { storage coefficient (storativity) of the entire aquifer } \\
& \mathrm{v} \equiv \text { hydraulic diffusivity }(\mathrm{T} / \mathrm{S}) \\
& \mathrm{r}_{\mathrm{w}} \equiv \text { well bore radius } \\
& \mathrm{r}_{\mathrm{i}} \equiv \text { influence radius }
\end{aligned}
$$

and the subscripts 1 and 2 refer to the least and most hydraulically diffusive intervals $\left(v_{2} / v_{1}>1\right)$. The latter three estimates tend to be more conservative. The safest strategy to minimize systematic errors due to transient effects is to wait as long as practical after pumping starts before taking borehole flow measurements. Dr. Fred Molz recommends waiting at least 30 minutes after pumping before starting borehole flowmeter testing (Molz, 2000). 
Tables 4 through 6 evaluate the four criteria for SWP-300A, SWP-200C and SWP-100D. In these tables, the "Ruud and Kabala (1996)" criterion refers to equation (6), "Molz and others (1989)" to equation (7), "Rehfeldt and others (1989)" to equation (5), and "Flach" refers to equation (8). Among these, criterion (6) based on Ruud and Kabala (1996) is preferred because it has a more rigorous basis than equations (5) and (7) and avoids the probable over-conservatism of equation (8). A reasonable assumption for diffusivity contrast is $100: 1$, considering that flows associated with intervals with conductivity lower than $1 / 100$ of the most permeable layer cannot be accurately measured. In evaluating hydraulic diffusivity (T/S), the transmissivity (T) and storage coefficient (S) estimates are based on the pumping tests of WSRC (1999, Table 5-1). Storage coefficients from pumping tests are more uncertain than transmissivity estimates for a heterogeneous aquifer (Meier and others, 1998; Sanchez-Vila and others, 1999). Therefore, the diffusivity estimates have similar uncertainty as the storage coefficient estimates.

The pre-test analysis indicates that quasi-steady flow conditions will conservatively occur within a minute or two for SWP-300A (Table 4), and within 30 minutes for SWP-200C (Table 5). These wells are in confined aquifer zones that have a high hydraulic diffusivity compared to unconfined aquifers (Table 1). SWP-100D is in the unconfined upper UTRA, which has low hydraulic diffusivity. Therefore, significantly longer times are required to reach quasi-steady conditions, as shown in Table 6. Assuming less conservative values for diffusivity contrast (e.g. 10:1), influence radius (e.g. $2 \mathrm{ft}$ ) and/or non-dimensional time $\left(t_{D}=4000\right)$ than for SWP-300A and SWP-200C produces an elapsed time under 2 hours for SWP-100D. This elapsed time is feasible for a single day test.

Bypass flow estimates: Head losses across the EBF during pumping can introduce significant errors in the hydraulic conductivity profile calculated from equations (3) or (4). Head loss causes some flow to bypass the EBF by through the formation and filter pack, and reduces the drawdown experienced by the formation below the EBF.

Bypass flow is undesirable because the EBF measures only a fraction of the flow leaving the aquifer below the instrument position, generally leading to an under-estimate of the actual conductivity. The exception occurs at the uppermost screen interval, when the EBF passes the top of the screen. Lacking screen above the EBF, bypass flow suddenly ceases and all flow must now pass through the meter. The sudden increase in differential flow for the top interval produces an anomalous high $\mathrm{K}$ estimate.

Reduced drawdown below the EBF are undesirable because equations (3) and (4) assume that the aquifer is exposed to the same drawdown over the entire well screen length. When the head loss caused by flow through the EBF is significant compared to the imposed drawdown, significant biases are introduced in the calculated relative conductivity profile. Dinwiddie and others (1999) demonstrated for a well without a filter pack that the lower three-quarters of the computed $\mathrm{K}$ profile is biased low while the upper quarter is biased high by a greater amount. They termed the phenomenon "head-lossinduced flow redistribution" and showed that the bias increases with increasing aquifer conductivity for the same pumping rate. 
Bypass flow calculations for the 1/2" and 1" EBF and various flowrates are included in the pre-test calculations shown in Tables 4 through 6 . The basis for the calculations is discussed in Flach and others (2000). Head losses can be practically eliminated by using the 1" ID EBF, or the 1/2" ID EBF at much lower flowrates. The estimated bypass flowrate using the 1" EBF is $1 \%$ of total flow at the top of the screen for pumping rates of 20, 15 and $15 \mathrm{~L} / \mathrm{min}$ for SWP-300A, SWP-200C and SWP-100D respectively. Head losses for the $1 / 2$ " EBF can be made small at practical pumping rates, but remain significant. For example, bypass flow is $5 \%$ of total flow using the $1 / 2$ " EBF and a pumping rate of $4 \mathrm{~L} / \mathrm{min}$ for SWP-200C. These pre-test calculations suggest that EBF testing be conducted using the 1" EBF and at pumping rates below 15-20 L/min (4-5 gpm).

\section{Field experience at the Southwest Plume Test Pad}

In addition to deploying the EBF, a conventional single-well pumping test was concurrently performed by using a pressure transducer and data logger to monitor drawdown before and during EBF testing. The time-drawdown data from pumping wells can be used to estimate average hydraulic conductivity independent of the prior multiplewell testing (WSRC, 1999). The purpose was to explore the feasibility of concurrent EBF and single-well pumping tests as a way to avoid the need for a prior estimate of average conductivity.

Prior EBF testing in D-area and R-area involved slotted well screens. The SWP wells have wire wrap screens with longitudinal ribs inside the screen. These internal ribs prevented a tight seal with the EBF packer and resulted in unanticipated and significant bypass flow inside the well casing. This bypass flow is in addition to flow bypassing the EBF outside the screen as discussed previously. Modifications to the normal calculation procedure were necessary to compensate, as discussed later in the report. Differential pressure between the packer and groundwater was held constant to ensure that bypass flow would be proportionally the same at all elevations.

Ambient flow testing was performed using the 1/2" EBF for greater sensitivity, and the 1" EBF was generally used for dynamic testing to minimize head loss. An electrical failure occurred with the 1" EBF during dynamic testing at SWP-200C. Testing with the 1" EBF was abandoned and restarted the next day with the 1/2" EBF at a lower flowrate. Unfortunately, head losses across the 1/2" EBF were significantly larger than for the 1" EBF, even at the lower pumping rate. The increased head loss caused large bypass flow between the packer bellows and the wire wrap screen.

\section{Conductivity distribution results}

Relative hydraulic conductivity profiles can be estimated from EBF ambient and dynamic flow data using equation (4). These results and interpretation of the ambient flow data are presented for each SWP well below.

SWP-300A: SWP-300A is a 4 inch well with a fully-penetrating $40 \mathrm{ft}$ screen in the Gordon aquifer. The EBF field data for SWP-300A are presented in Table 7. Ambient 
and dynamic testing were performed at $1 \mathrm{ft}$ intervals. Dynamic testing began 32 minutes after pumping, which easily exceeds the pre-test criteria for quasi-steady flow of 2 minutes (Table 4). The occurrence of bypass flow between the packer bellows and wire wrap screen is evident in both the ambient and dynamic testing results from measurements taken near joints between screen sections. At the joint between two screen sections, a good seal was apparently achieved with the EBF packer. Significantly higher flows were recorded during dynamic testing at $10 \mathrm{ft}$ intervals corresponding to screen section joints. Comparing measured flow at a joint to the average of flows just above and below the joint indicate that the EBF measured approximately $70 \%$ of total discharge and $30 \%$ bypassed the EBF.

The field data are analyzed at 1, 2, 5 and $10 \mathrm{ft}$ resolutions in Tables 8 through 11 . Analysis of the data at intervals greater than $1 \mathrm{ft}$ reduces noise/uncertainty in the relative conductivity profile, at the expense of resolution. The calculations at a $10 \mathrm{ft}$ interval have the added benefit of eliminating the need to correct for internal bypass flow, because only measurements at screen joints are involved.

Column (4) in Tables 8 through 11 lists flow inside the well casing under ambient conditions, measured as a function position with the EBF. A positive value indicates upward flow, and a negative value corresponds to downward flow. The ambient flow data have not been adjusted to account for internal bypass flow because conductivity characterization is the main objective of this study and the ambient flows are insignificant compared to dynamic flows. Fortunately, the data at $10 \mathrm{ft}$ intervals require no correction and can be interpreted at face value. Overall the ambient data at $10 \mathrm{ft}$ increments indicate downward flow through the casing due to a downward head gradient in the aquifer (column (4) of Table 11). Column (5) lists differential ambient flow, computed as the difference between adjacent EBF measurements. In this column, a positive value means groundwater is entering the casing (leaving the aquifer) while a negative value means water is flowing out the screen (entering the aquifer). These data are plotted in Figure 7. For SWP-300A, groundwater is observed to enter the casing over the upper three-quarters of the screen and leave over the bottom quarter.

The non-dimensional or relative hydraulic conductivity distribution, $\mathrm{K}_{\mathrm{i}} / \mathrm{K}$, based on EBF data alone is shown in column (15) (column (13) in Table 11). The negative values observed at 1 and $2 \mathrm{ft}$ analysis resolution are not physically possible of course. Negative values of $\mathrm{K}_{\mathrm{i}} / \mathrm{K}$, are generally the result of measurement errors and non-ideal test conditions. Expanding on the latter, any artificial reduction in EBF flow when the instrument is passing by a low permeability interval can cause a negative differential net flow in column (11) (column (9) in Table 11). Examples include a reduction in pump flowrate, which is assumed to be constant in the analysis, and increased flow bypassing the EBF internally or through the filter pack, which also reduces EBF measured flow. These reductions in EBF measured flow are typically small, but can overwhelm the true differential net flow from the aquifer over an interval if the permeability is low. Therefore, negative values should simply be interpreted as a low permeability zone, and/or an artifact of varying internal bypass flow. At the coarser 5 and $10 \mathrm{ft}$ resolutions, 
conductivities are all positive. The amount of flow bypassing the EBF through the filter pack is estimated to be negligible.

The relative conductivity profile for SWP-300A is plotted in Figure 8 for 1, 2, 5 and $10 \mathrm{ft}$ intervals. Values greater than 1 correspond to greater than average conductivity, and vice versa. Two high conductivity zones separated by a low conductivity interval are observed in the lower half of the screen. Conductivity is relatively low in the upper half of the screen.

SWP-200C: SWP-200C is a 6 inch well with a fully-penetrating $60 \mathrm{ft}$ screen in the lower UTRA. The EBF field data for SWP-200C are presented in Table 12. Ambient testing was performed at $1 \mathrm{ft}$ intervals using the 1/2" EBF. Subsequent dynamic testing was performed at $2 \mathrm{ft}$ intervals to save time, also using the 1/2" EBF due to equipment failure with the 1" EBF. Dynamic testing began 37 minutes after pumping, which exceeds the pre-test criteria for quasi-steady flow of 30 minutes (Table 5). Apparently none of the test elevations aligned with a joint between screens, as intermediate high flows were not observed as with SWP-300A. However, measured flow increased abruptly as the EBF passed the top of screen in going from a depth of 92.5 to $90.5 \mathrm{ft}$. At this point the measured EBF flow was only $11 \%$ of the total, compared to about $70 \%$ for SWP-300A. The increased internal bypass flow is a result of greater head loss associated with the 1/2" EBF.

The field data are analyzed at 2 and $6 \mathrm{ft}$ resolutions in Tables 13 and 14. Analysis of the data at $6 \mathrm{ft}$ intervals reduces noise/uncertainty in the relative conductivity profile, at the expense of resolution. The ambient flow data have not been adjusted to account for internal bypass flow because conductivity characterization is the main objective of this study and the ambient flows are insignificant compared to dynamic flows. Nevertheless, differential ambient flow at a $6 \mathrm{ft}$ resolution is plotted in Figure 9. Roughly speaking, flow enters the upper portion of the screen and exits the lower portion, consistent with a downward head gradient. The non-dimensional or relative hydraulic conductivity distribution, $\mathrm{K}_{\mathrm{i}} / \mathrm{K}$, based on $\mathrm{EBF}$ data alone is shown in column (15) of both tables. The presence of significant negative conductivities in the upper portion of the screen suggests significant measurement errors along that portion of the screen. The relative conductivity profile for SWP-200C is plotted in Figure 10 for 2 and $6 \mathrm{ft}$ intervals. Values greater than 1 correspond to greater than average conductivity, and vice versa. Two high conductivity zones separated by a low conductivity interval are observed in the middle of the screen zone. Bypass flow through the filter pack is non-negligible, as shown by column (20) in the tables.

SWP-100D: SWP-100D is a 6 inch well with a fully-penetrating $30 \mathrm{ft}$ screen in the upper UTRA. The EBF field data for SWP-100D are presented in Table 15. Ambient testing was performed at $1 \mathrm{ft}$ intervals using the 1/2" EBF. Dynamic testing was performed at 1 ft intervals using the 1" EBF. Dynamic testing was performed between 76 and 145 minutes after pumping, which is generally shorter than the pre-test objective 2 hours (Table 6). Therefore some bias due to transient effects is probable. At a TOC depth of $67.8 \mathrm{ft}$, the EBF apparently aligned with a joint between screens, as measured flow 
increased abruptly compared to the test stations immediately above and below. At this point the measured EBF flow was estimated to be $48 \%$ of the total.

The field data are analyzed at 1, 3 and $7 \mathrm{ft}$ resolutions in Tables 16 through 18 . As before the ambient flow data have not been adjusted to account for internal bypass flow. Nevertheless, differential ambient flow at a $7 \mathrm{ft}$ resolution is plotted in Figure 11. The result appears to be non-physical in that flow is only shown entering the screen. Under steady conditions, this is impossible. Measurement error, or possibly a rising water table, are probably the cause. The non-dimensional or relative hydraulic conductivity distribution, $\mathrm{K}_{\mathrm{i}} / \mathrm{K}$, based on $\mathrm{EBF}$ data alone is shown in column (15) of the tables. Some negative values of conductivity are observed at a $1 \mathrm{ft}$ resolution, but not the coarser resolutions. The relative conductivity profile for SWP-100D is plotted in Figure 12 for 1, 3 and $7 \mathrm{ft}$ intervals. Values greater than 1 correspond to greater than average conductivity, and vice versa. Conductivity generally increases from top to bottom of the screen. Bypass flow through the filter pack is negligible, as shown by column (20) in the tables.

Comparison to natural gamma log: Figure 13 compares the relative hydraulic conductivity profiles derived from EBF testing to a natural gamma log from nearby borehole SWP01SB. Intervals of low gamma response tend to correlate with intervals of high hydraulic conductivity, consistent with the presence of sandy sediment. However, reproducing in detail the conductivity variation inferred from EBF testing is not feasible. The comparison illustrates the value of EBF testing when accurate knowledge of conductivity variations is needed.

\section{Average conductivity estimates}

Dimensional conductivity profiles can be obtained by multiplying the relative conductivity profile from EBF testing (e.g. Figures 8, 10 and 12) by an independent estimate of average conductivity over the screen zone. The SWP wells are fullypenetrating, so the screen-average conductivity is synonymous with formation-average conductivity. Conductivity averaged over formation thickness can be estimated from multiple- or single-well aquifer testing.

Prior multiple-well testing: The results of prior multiple-well aquifer testing at the Southwest Plume Test Pad are presented by WSRC (1999) and summarized in Tables 1 and 19. These conductivity estimates are more reliable than single-well aquifer test results because well losses in the pumping well do not affect drawdowns in the observation wells. Tables 8-11, 13-14 and 16-18 incorporate these average conductivity estimates in the dimensional conductivity profiles.

Single-well testing concurrent with EBF testing: Single-well aquifer testing was performed concurrently with EBF testing to assess the feasibility of estimating average conductivity in this manner at sites where prior estimates are not available. Appendices $\mathrm{A}, \mathrm{B}$ and $\mathrm{C}$ contain the water level transducer and barometric pressure data and plots for SWP-300A, 200C and 100D, respectively. Although well efficiencies are known from prior multiple-well testing (Table 1), these estimates are ignored in the present analysis so 
that estimates independent of prior testing can be developed. Instead, the efficiency of each well is assumed to be $50 \%$, a value judged to be representative of average conditions. Figures 14 through 16 illustrate time-drawdown data for each well, after applying the assumed well efficiency of 50\%. The average transmissivity values listed in these figures were estimated using AQTESOLV using the type curve denoted in each figures. Only the early time data before EBF testing began are analyzed in these figures. Hence these single-well pumping tests were of short duration. Emphasis was placed on accurately fitting the longer times in each set of drawdown data to reduce the effects of well bore storage. The corresponding conductivity estimates, based on saturated formation thickness from Table 1, are compared to the prior multiple-well results in Table 19. Significant differences are observed between the multiple- and single-well test results, largely because the assumed well efficiency of $50 \%$ is inaccurate. This exercise illustrates the well-known difficulty in predicting conductivity from single-well aquifer tests.

\section{Alternative conductivity estimates using Cooper-Jacob analysis}

Horizontal conductivity can be estimated directly for individual screen intervals, provided the pumping rate is held constant (or is the superposition of constant pumping rates) and time-drawdown data are recorded in addition to EBF flowrates. Under these conditions, a Cooper-Jacob analysis can be applied to each screen interval as summarized by equation (3). Well losses can be introduced by modifying equation (3) as

$$
\mathrm{K}_{\mathrm{i}}=\frac{\left(\Delta \mathrm{Q}_{\mathrm{i}}-\Delta \mathrm{q}_{\mathrm{i}}\right)}{2 \pi \Delta \mathrm{z}_{\mathrm{i}} \Delta \mathrm{hE}} \ln \left(\frac{1.5}{\mathrm{r}_{\mathrm{w}}} \sqrt{\frac{\mathrm{Tt}}{\mathrm{S}}}\right)
$$

where $\mathrm{E}$ is well efficiency, defined to be the theoretical drawdown divided by actual drawdown $(\Delta \mathrm{h})$. If barometric pressure variations are significant, further adjustments to measured drawdown are needed. This approach replaces the need for a prior screenaverage conductivity estimate, ideally from a separate multiple-well pumping test, with single-well pump testing conducted concurrently with EBF flow measurements. Being a form of single-well aquifer testing, the Cooper-Jacob analysis requires prior estimates for hydraulic diffusivity (T/S) and well efficiency (E). Transmissivity (T) can be determined iteratively from the layer estimates of conductivity $\left(\mathrm{K}_{\mathrm{i}}\right)$. Therefore, storage coefficient $(\mathrm{S})$ and well efficiency (E) are fundamentally required. Both are difficult to estimate with accuracy. However, the Cooper-Jacob estimate is not highly sensitive to the assumed value of storage coefficient for large times, because $S$ appears within a square-root and logarithm in equation (9). Unfortunately, the same cannot be said for well efficiency because conductivity is inversely proportional to E. Therefore, EBF conductivity estimates from equation (9) have a level of uncertainty similar to conventional single-well pumping test estimates.

The conditions required for Cooper-Jacob analysis were satisfied during EBF testing at all 3 SWP wells. Barometric pressure was monitored, but did not vary substantially over the course of a 2 hour EBF test. Therefore, no barometric corrections to the time-drawdown data were made. Prior to EBF testing, water level changed smoothly with time. During 
EBF testing, packer inflation and deflation, and movement of the EBF, caused artificial fluctuations in water level. Unlike the conventional single-well analysis discussed above, the Cooper-Jacob analysis is applied during EBF testing. Therefore the water level transducer data must be smoothed to remove these artifacts from the time-drawdown data. Figures 17 through 19 plot the water level transducer response in the unaltered and smoothed forms. At SWP-200C the flowrate was increased from about $4 \mathrm{~L} / \mathrm{min}$ to about $7 \mathrm{~L} / \mathrm{min}$ after 2 hours of pumping, creating an abrupt change in transducer pressure at that time. Borehole flowrates near the bottom of SWP-200C at a pumping rate of $4 \mathrm{~L} / \mathrm{min}$ were too small to be resolved by the 1" $\mathrm{EBF}$, so the pumping rate was increased. At SWP-100D the transducer was raised $2 \mathrm{ft}$ near the end of EBF testing so that the pump and EBF could be raised as close as possible to the water level. The offset is accounted for by raising the smoothed tail of the unaltered transducer response by $2 \mathrm{ft}$. Tables $8-11$, 13-14 and 16-18 use the smoothed drawdown data to compute dimensional conductivity profiles using the Cooper-Jacob analysis. For these calculations, well efficiency was assumed to be $50 \%$ as with conventional single-well testing. Specific storage coefficient were assumed to be $10^{-5} \mathrm{ft}^{-1}$ for confined aquifers and $10^{-2} \mathrm{ft}^{-1}$ for unconfined aquifers.

The Cooper-Jacob conductivity estimates are shown in the second half of the calculation tables. The ratio of the Cooper-Jacob estimate to the estimate based on prior multiplewell aquifer testing is shown in each table. The ratio varies little, which means that the relative conductivity variation is the practically the same between the two calculation methods. Similarly, the Cooper-Jacob estimates are averaged over the screen zone and compared to the prior multiple-well test result. The average Cooper-Jacob estimates are included in Table 19 for comparison to the prior multiple-well and conventional singlewell aquifer test results. Significant differences are observed between the multiple-well and average Cooper-Jacob results, largely because the assumed well efficiency of $50 \%$ is inaccurate. When well efficiency and specific storage are based on the prior multiplewell aquifer tests (WSRC, 1999), the agreement is much improved.

\section{Considerations and recommendations for future EBF deployment}

Based on the experience gained at the Southwest Plume Test Pad, the following recommendations and considerations for future $\mathrm{EBF}$ deployments are stated.

1 " versus 1/2" EBF for dynamic testing: As suggested by Flach and others (2000), the 1" EBF is clearly preferred over the 1/2" EBF for dynamic testing. The 1" EBF has more than adequate sensitivity and practically eliminates bypass flow through the filter pack.

Slotted versus wire wrap screen: The EBF packer cannot achieve a good seal against a wire wrap screen, which leads to undesirable internal bypass flow, even with the 1" EBF. The measured EBF flow can be corrected if measurements are taken at screen section joints and above the top of screen. At these locations a good seal is achieved and the measured flow provides a good reference for corrections to the surrounding data. Because of variations in the packer seal against a wire wrap screen, the corrections are imperfect and introduce noticeable noise in the relative conductivity profiles. The noise can be reduced by analyzing the data at a coarser resolution, but at the expense of also 
smoothing out real conductivity variations. Other considerations being equal, slotted screen is preferred over wire wrap screen for the purpose of measuring flowrates with the EBF. If single-well aquifer testing is to be performed concurrently with EBF testing, a wire wrap screen might be more desirable from the standpoint of increased well efficiency however.

Equipment issues: The present EBF system has a number of shortcomings that should ideally be remedied before extensive, routine application of the technology at the SRS. Several deficiencies have been identified in Flach and others (2000) in reference to R-area EBF testing. At the Southwest Plume Test Pad, the 1" EBF failed electrically causing lost time and poorer quality results as a result of subsequently having to use the $1 / 2$ " EBF. A more reliable EBF system is needed.

Feasibility of concurrent single-well aquifer testing: Single-well aquifer testing performed concurrently with EBF testing was investigated as an possible alternative to reliance on prior multiple-well estimates of average horizontal conductivity or well efficiency. Single-well testing refers to conventional estimates of screen-average conductivity and estimates of subinterval conductivity using Cooper-Jacob analysis. As with all single-well testing, either approach depends on an uncertain estimate of well efficiency in the absence of prior multiple-well testing. Concurrent single-well aquifer testing was determined to be feasible in the field. Based on EBF testing in R-area and at the Southwest Plume Test Pad, conductivity estimates based on single-well testing alone have an uncertainty of roughly plus or minus a factor of 2 . A prior multiple-well estimate of average conductivity is technically preferred, but concurrent single-well testing may be preferred overall considering the cost of multiple-well aquifer testing. 


\section{References}

Cooper, H. H. and C. E. Jacob, 1946, A generalized graphical method for evaluating formation constants and summarizing well-field history, Transactions of the American Geophysical Union, v27, 526-534.

Dinwiddie, C. L., N. A. Foley and F. J. Molz, 1999, In-well hydraulics of the Electromagnetic Borehole Flowmeter, Ground Water, v37 n2, 305-315.

Flach, G. P., F. C. Sappington, W. Pernell Johnson and R. A. Hiergesell, 2000, Electromagnetic Borehole Flowmeter (EBF) testing in R-area (U), WSRC-TR-2000-00170.

Javendel, I., and P. A. Witherspoon, 1969, A method for analyzing transient flow in multilayered aquifers, Water Resources Research, v5 n4, 856-869.

Meier, P. M., J. Carrera and X. Sanchez-Vila, 1998, An evaluation of Jacob's method for the interpretation of pumping tests in heterogeneous formations, Water Resources Research, v34 n5, 1101-1025.

Molz, F. J., 2000, personal communication, e-mail dated 7/12.

Molz, F. J., R. H. Morin, A. E. Hess, J. G. Melville and O. Guven, 1989, The impeller meter for measuring aquifer permeability variations: evaluation and comparison with other tests, Water Resources Research, v25 n7, 1677-1683.

Molz, F. J. and S. C. Young, 1993, Development and application of borehole flowmeters for environmental assessment, The Log Analyst, v3, Jan.-Feb., 13-23.

Rehfeldt, K. R., P. Hufschmied, L. W. Gelhar and M. E. Schaefer, 1989, The borehole flowmeter technique for measuring hydraulic conductivity variability, report, Electric Power Research Institute, Palo Alto CA.

Ruud, N. C., and Z. J. Kabala, 1996, Numerical evaluation of flowmeter test interpretation methodologies, Water Resources Research, v32 n4, 845-852.

Sanchez-Vila, X., P. M. Meier and J. Carrera, 1999, Pumping tests in heterogeneous aquifers: An analytical study of what can be obtained from their interpretation using Jacob's method, Water Resources Research, v35 n4, 943-952.

Waldrop, W. R., 1995, A summary of hydrogeologic studies with the Electromagnetic Borehole Flowmeter, report QEC T-102, Quantum Engineering Corporation, 112 Tigitsi Lane, Loudon, Tennessee, 37774, 615-458-0506.

WSRC, 1999, Aquifer testing results from the Burial Ground Complex (U); Book 1 of 2; Southwest Plume Test Pad, WSRC-RP-99-4069. 
Young, S. C. and W. R. Waldrop, 1989, An electromagnetic borehole flowmeter for measuring hydraulic conductivity variability, in Molz, F. J., O. Guven and J. G. Melville eds., Proceedings of the conference on new field techniques for quantifying the physical and chemical properties of heterogeneous aquifers: Water Resources Research Institute, Auburn University, Alabama. 


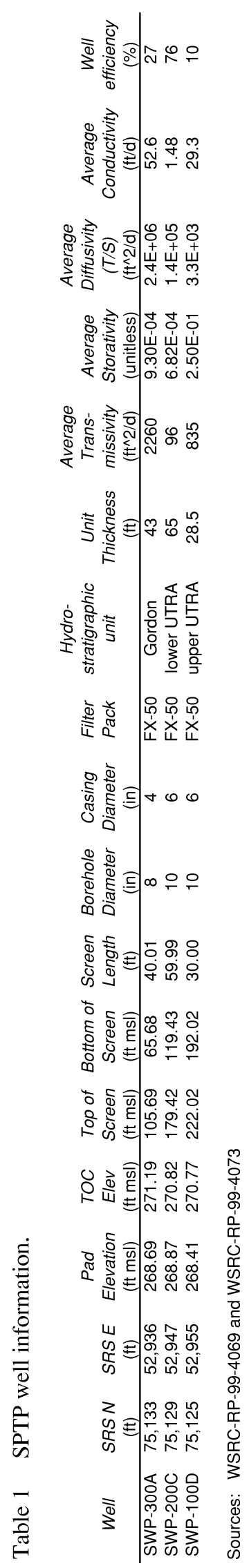




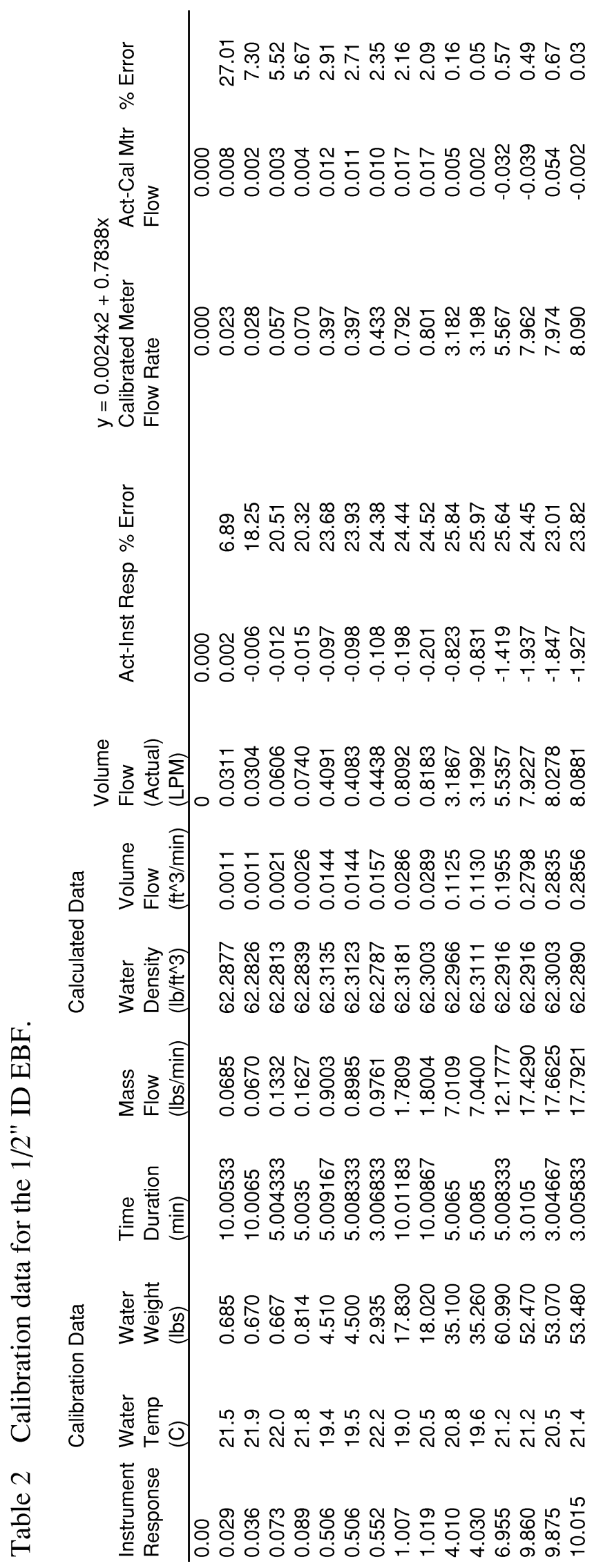




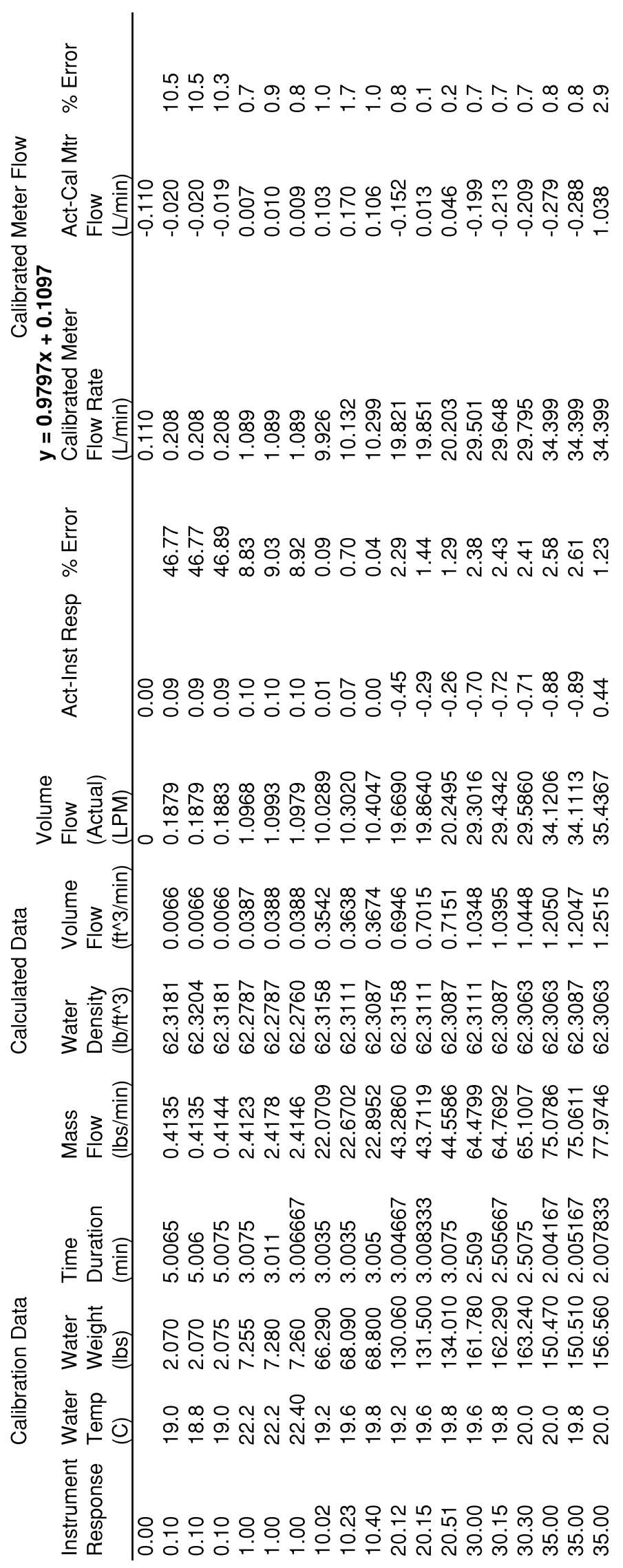


Table 4 Pre-test design calculations for SWP-300A.

Pretest criteria for valid EBF application (pseudo-steady state \& minimal bypass flow)

Site

Formation properties estimated $\mathrm{K}(\mathrm{ft} / \mathrm{d}) \quad 52.6$

estimated $\mathrm{S}_{\mathrm{S}}(1 / \mathrm{ft}) \quad 2.2 \mathrm{E}-05$

thickness $b(\mathrm{ft}) \quad 43$

estimated $\mathrm{T}\left(\mathrm{ft}^{2} / \mathrm{d}\right) \quad 2261.8$ estimated $S$ 9.3E-04

diffusivity $v=\mathrm{T} / \mathrm{S}\left(\mathrm{ft}^{2} / \mathrm{d}\right) \quad 2.4 \mathrm{E}+06$

Well properties

borehole diameter (in) 8

Casing diameter (in) 4

Borehole radius (in) 4

Casing radius (in) 2

Borehole radius $(\mathrm{m}) \quad 0.1016$

Casing radius $(\mathrm{m}) \quad 0.0508$

Annulus area $\left(\mathrm{m}^{2}\right) \quad 0.024$

Annulus thickness $(\mathrm{cm}) \quad 5.08$

Filter pack conductivity (m/d) 100

\section{EBF properties}

$$
\mathrm{L}_{\mathrm{EBF}}(\mathrm{m}) \quad 0.178
$$

Bypass properties

$$
\begin{array}{rr}
\Delta \mathrm{L}(\mathrm{m}) & 0.079 \\
\mathrm{~L}(\mathrm{~m}) & 0.257
\end{array}
$$

\begin{tabular}{|c|c|c|c|c|}
\hline \multicolumn{5}{|c|}{ Ruud and Kabala (1996) pseudo-steady criterion } \\
\hline \multicolumn{5}{|c|}{$1.0 \mathrm{E}+03$ Non-dimensional time, $t_{0}$} \\
\hline $\begin{array}{c}\text { Diffusivity } \\
\text { contrast }\end{array}$ & $\begin{array}{l}\text { Time, } t \\
\text { (d) }\end{array}$ & $\begin{array}{c}\text { Time, t } \\
\text { (hr) }\end{array}$ & $\begin{array}{l}\text { Time, } \mathrm{t} \\
\text { (min) }\end{array}$ & $\begin{array}{c}\text { Time, } t \\
(s)\end{array}$ \\
\hline $1: 1$ & 0.0 & 0.0 & 0.0 & 1.0 \\
\hline $10: 1$ & 0.0 & 0.0 & 0.1 & 5.4 \\
\hline $100: 1$ & 0.0 & 0.0 & 0.8 & 50.0 \\
\hline $1000: 1$ & 0.0 & 0.1 & 8.3 & 495.6 \\
\hline
\end{tabular}

Molz and others (1989) pseudo-steady criterion (but using assumed influence radius per personal comm.) 1.0E+02 Non-dimensional time, $t_{D}$ Influence Time, $\mathrm{t}$ Time, $\mathrm{t}$ Time, $\mathrm{t}$ Time, $\mathrm{t}$

\begin{tabular}{ccccr} 
radius, $r(\mathrm{ft})$ & $(\mathrm{d})$ & $(\mathrm{hr})$ & $(\mathrm{min})$ & \multicolumn{1}{c}{$(\mathrm{s})$} \\
\hline 1 & 0.0 & 0.0 & 0.0 & 0.9 \\
2 & 0.0 & 0.0 & 0.1 & 3.6 \\
5 & 0.0 & 0.0 & 0.4 & 22.3 \\
10 & 0.0 & 0.0 & 1.5 & 89.1 \\
20 & 0.0 & 0.1 & 5.9 & 356.4
\end{tabular}

\section{Rehfeldt and others (1989) and Flach criteria} Non-

dimensional Time, $\mathrm{t}$ Time, $\mathrm{t}$ Time, $\mathrm{t}$ Time, $\mathrm{t}$

\begin{tabular}{ccccrc} 
time, $\mathrm{t}_{\mathrm{D}}$ & $(\mathrm{d})$ & $(\mathrm{hr})$ & $(\mathrm{min})$ & $(\mathrm{s})$ & \\
\cline { 1 - 5 } $4.0 \mathrm{E}+02$ & 0.0 & 0.0 & 0.0 & 0.4 & $10: 1$ \\
$4.0 \mathrm{E}+03$ & 0.0 & 0.0 & 0.1 & 4.0 & $100: 1$ \\
$4.0 \mathrm{E}+04$ & 0.0 & 0.0 & 0.7 & 39.6 & $\mathrm{r} / \mathrm{b}<0.125$ \\
$1.0 \mathrm{E}+06$ & 0.0 & 0.3 & 16.5 & 990.1 & long time
\end{tabular}

\begin{tabular}{cccccc}
\multicolumn{2}{c}{ Bypass flow estimate } & & & \\
1/2" EBF & & 1" EBF & \\
$\begin{array}{c}\text { Pumping } \\
\text { rate, QP } \\
\text { (L/min) }\end{array}$ & $\begin{array}{c}\text { Pumping } \\
\text { rate, QP } \\
\text { (gal/min) }\end{array}$ & $\begin{array}{c}\text { Bypass } \\
(\mathrm{L} / \mathrm{min})\end{array}$ & Ratio & $\begin{array}{c}\text { Bypass } \\
(\mathrm{L} / \mathrm{min})\end{array}$ & Ratio \\
\hline 0.2 & 0.05 & 0.00 & $0.8 \%$ & 0.00 & $0.1 \%$ \\
0.5 & 0.13 & 0.01 & $1.1 \%$ & 0.00 & $0.1 \%$ \\
1 & 0.26 & 0.01 & $1.4 \%$ & 0.00 & $0.1 \%$ \\
2 & 0.53 & 0.04 & $2.2 \%$ & 0.00 & $0.1 \%$ \\
5 & 1.32 & 0.23 & $4.6 \%$ & 0.01 & $0.3 \%$ \\
10 & 2.64 & 0.86 & $8.6 \%$ & 0.05 & $0.5 \%$ \\
20 & 5.28 & 3.29 & $16.4 \%$ & 0.21 & $1.0 \%$ \\
\hline 20 & 5.28 & 3.29 & $16.4 \%$ & 0.21 & $1.0 \%$
\end{tabular}


Table 5 Pre-test design calculations for SWP-200C.

Pretest criteria for valid EBF application (pseudo-steady state \& minimal bypass flow)

Site

SWP-200C

Formation properties

estimated $\mathrm{K}(\mathrm{ft} / \mathrm{d}) \quad 1.48$

estimated $S_{\mathrm{S}}(1 / \mathrm{ft}) \quad 1.0 \mathrm{E}-05$

thickness $\mathrm{b}(\mathrm{ft}) \quad 65$

estimated $\mathrm{T}\left(\mathrm{ft}^{2} / \mathrm{d}\right) \quad 96.2$ estimated $\mathrm{S} \quad 6.8 \mathrm{E}-04$

diffusivity $v=\mathrm{T} / \mathrm{S}\left(\mathrm{ft}^{2} / \mathrm{d}\right) \quad 1.4 \mathrm{E}+05$

\section{Well properties}

borehole diameter (in) 10

Casing diameter (in) 6

Borehole radius (in) 5

Casing radius (in) 3

Borehole radius $(\mathrm{m}) \quad 0.1270$

Casing radius $(\mathrm{m}) \quad 0.0762$

Annulus area $\left(\mathrm{m}^{2}\right) \quad 0.032$

Annulus thickness $(\mathrm{cm}) \quad 5.08$

Filter pack conductivity (m/d) 100

\section{EBF properties}

$$
L_{E B F}(m) \quad 0.178
$$

Bypass properties

$$
\begin{array}{rr}
\Delta \mathrm{L}(\mathrm{m}) & 0.079 \\
\mathrm{~L}(\mathrm{~m}) & 0.257
\end{array}
$$

\begin{tabular}{ccccr} 
Ruud and Kabala (1996) & pseudo-steady criterion \\
1.0E+03 & Non-dimensional time, $\mathrm{t}_{\mathrm{D}}$ & \\
Diffusivity & Time, $\mathrm{t}$ & Time, $\mathrm{t}$ & Time, $\mathrm{t}$ & Time, $\mathrm{t}$ \\
contrast & $(\mathrm{d})$ & $(\mathrm{hr})$ & $(\mathrm{min})$ & \multicolumn{1}{c}{$(\mathrm{s})$} \\
\hline $1: 1$ & 0.0 & 0.0 & 0.4 & 26.4 \\
$10: 1$ & 0.0 & 0.0 & 2.4 & 144.9 \\
$100: 1$ & 0.0 & 0.4 & 22.2 & 1330.7 \\
$1000: 1$ & 0.2 & 3.7 & 219.8 & 13188.9
\end{tabular}

Molz and others (1989) pseudo-steady criterion

(but using assumed influence radius per personal comm.)

1.0E+02 Non-dimensional time, $t_{D}$

Influence Time, $\mathrm{t}$ Time, $\mathrm{t}$ Time, $\mathrm{t}$ Time, $\mathrm{t}$

\begin{tabular}{ccccr} 
radius, $r(\mathrm{ft})$ & $(\mathrm{d})$ & $(\mathrm{hr})$ & $(\mathrm{min})$ & \multicolumn{1}{c}{$(\mathrm{s})$} \\
\hline 1 & 0.0 & 0.0 & 0.3 & 15.2 \\
2 & 0.0 & 0.0 & 1.0 & 60.7 \\
5 & 0.0 & 0.1 & 6.3 & 379.5 \\
10 & 0.0 & 0.4 & 25.3 & 1517.8 \\
20 & 0.1 & 1.7 & 101.2 & 6071.4
\end{tabular}

\section{Rehfeldt and others (1989) and Flach criteria} Non-

dimensional Time, $\mathrm{t}$ Time, $\mathrm{t}$ Time, $\mathrm{t}$ Time, $\mathrm{t}$

\begin{tabular}{ccccrcc} 
time, $\mathrm{t}_{\mathrm{D}}$ & $(\mathrm{d})$ & $(\mathrm{hr})$ & $(\mathrm{min})$ & \multicolumn{1}{c}{$(\mathrm{s})$} & \\
\cline { 1 - 5 } $4.0 \mathrm{E}+02$ & 0.0 & 0.0 & 0.2 & 10.5 & $10: 1$ \\
$4.0 \mathrm{E}+03$ & 0.0 & 0.0 & 1.8 & 105.4 & $100: 1$ \\
$4.0 \mathrm{E}+04$ & 0.0 & 0.3 & 17.6 & 1054.1 & $\mathrm{r} / \mathrm{b}<0.125$ \\
$1.0 \mathrm{E}+06$ & 0.3 & 7.3 & 439.2 & 26351.4 & long time
\end{tabular}

\begin{tabular}{cccccc}
$\begin{array}{c}\text { Bypass flow estimate } \\
\text { 1/2" EBF }\end{array}$ & \multicolumn{3}{c}{ 1" EBF } \\
$\begin{array}{c}\text { Pumping } \\
\text { rate, QP } \\
\text { (L/min) }\end{array}$ & $\begin{array}{c}\text { Pumping } \\
\text { rate, QP }\end{array}$ & $\begin{array}{c}\text { Bypass } \\
\text { (gal/min) }\end{array}$ & $\begin{array}{c}\text { B } / \text { min) } \\
\text { Bypass }\end{array}$ & \\
\hline 0.2 & 0.05 & 0.00 & $1.1 \%$ & 0.00 & $0.1 \%$ \\
0.5 & 0.13 & 0.01 & $1.4 \%$ & 0.00 & $0.1 \%$ \\
1 & 0.26 & 0.02 & $1.9 \%$ & 0.00 & $0.1 \%$ \\
2 & 0.53 & 0.06 & $3.0 \%$ & 0.00 & $0.2 \%$ \\
5 & 1.32 & 0.31 & $6.1 \%$ & 0.02 & $0.4 \%$ \\
10 & 2.64 & 1.14 & $11.4 \%$ & 0.07 & $0.7 \%$ \\
20 & 5.28 & 4.38 & $21.9 \%$ & 0.27 & $1.4 \%$ \\
\hline 15 & 3.96 & 2.50 & $16.7 \%$ & 0.16 & $1.0 \%$ \\
4 & 1.06 & 0.20 & $5.1 \%$ & 0.01 & $0.3 \%$
\end{tabular}


Table 6 Pre-test design calculations for SWP-100D.

Pretest criteria for valid EBF application (pseudo-steady state \& minimal bypass flow)

Site

SWP-100D

Formation properties estimated K (ft/d) 29.3

estimated $S_{s}(1 / f t)$ thickness $b(\mathrm{ft})$

8.8E-03 estimated $\mathrm{T}\left(\mathrm{ft}^{2} / \mathrm{d}\right) \quad 835$ estimated $S \quad 2.5 \mathrm{E}-01$ diffusivity $v=\mathrm{T} / \mathrm{S}\left(\mathrm{ft}^{2} / \mathrm{d}\right) \quad 3.3 \mathrm{E}+03$

Well properties

borehole diameter (in) 10 Casing diameter (in) 6

Borehole radius (in) 5

Casing radius (in) 3

Borehole radius $(\mathrm{m}) \quad 0.1270$

Casing radius $(\mathrm{m}) \quad 0.0762$

Annulus area $\left(\mathrm{m}^{2}\right) \quad 0.032$

Annulus thickness $(\mathrm{cm}) \quad 5.08$

Filter pack conductivity (m/d) 100

\section{EBF properties}

$$
L_{E B F}(m) \quad 0.178
$$

Bypass properties

$$
\begin{array}{rr}
\Delta \mathrm{L}(\mathrm{m}) & 0.079 \\
\mathrm{~L}(\mathrm{~m}) & 0.257
\end{array}
$$

\begin{tabular}{ccccc} 
Ruud and Kabala (1996) & pseudo-steady criterion \\
1.0E +03 & Non-dimensional time, $\mathrm{t}_{\mathrm{D}}$ & \\
Diffusivity & Time, $\mathrm{t}$ & Time, $\mathrm{t}$ & Time, $\mathrm{t}$ & Time, $\mathrm{t}$ \\
contrast & (d) & (hr) & (min) & (s) \\
\hline $1: 1$ & 0.0 & 0.3 & 18.7 & 1119.9 \\
$10: 1$ & 0.1 & 1.7 & 102.7 & 6159.3 \\
$100: 1$ & 0.7 & 15.7 & 942.6 & 56554.0 \\
$1000: 1$ & 6.5 & 155.7 & 9341.7 & \#\#\#\#\#\#
\end{tabular}

\section{Molz and others (1989) pseudo-steady criterion}

(but using assumed influence radius per personal comm.)

\begin{tabular}{|c|c|c|c|c|}
\hline radius, $r(\mathrm{ft})$ & (d) & $(\mathrm{hr})$ & (min) & (s) \\
\hline 1 & 0.0 & 0.2 & 10.8 & 645.1 \\
\hline 2 & 0.0 & 0.7 & 43.0 & 2580.2 \\
\hline 5 & 0.2 & 4.5 & 268.8 & 16126.3 \\
\hline 10 & 0.7 & 17.9 & 1075.1 & 64505.1 \\
\hline 20 & 3.0 & 71.7 & 4300.3 & \#\#\#\#\#\#\# \\
\hline
\end{tabular}

1.0E+02 Non-dimensional time, $t_{D}$

Influence Time, $\mathrm{t}$ Time, $\mathrm{t}$ Time, $\mathrm{t}$ Time, $\mathrm{t}$

\section{Rehfeldt and others (1989) and Flach criteria} Non-

\begin{tabular}{|c|c|c|c|c|c|}
\hline \multicolumn{2}{|c|}{ Bypass flow estimate } & \multicolumn{2}{|l|}{$1 / 2 " E B F$} & \multicolumn{2}{|l|}{$1 " E B F$} \\
\hline $\begin{array}{c}\text { Pumping } \\
\text { rate, QP } \\
\text { (L/min) }\end{array}$ & $\begin{array}{l}\text { Pumping } \\
\text { rate, QP } \\
\text { (gal/min) }\end{array}$ & $\begin{array}{l}\text { Bypass } \\
\text { (L/min) }\end{array}$ & Ratio & $\begin{array}{l}\text { Bypass } \\
\text { (L/min) }\end{array}$ & Ratio \\
\hline 0.2 & 0.05 & 0.00 & $1.1 \%$ & 0.00 & $0.1 \%$ \\
\hline 0.5 & 0.13 & 0.01 & $1.4 \%$ & 0.00 & $0.1 \%$ \\
\hline 1 & 0.26 & 0.02 & $1.9 \%$ & 0.00 & $0.1 \%$ \\
\hline 2 & 0.53 & 0.06 & $3.0 \%$ & 0.00 & $0.2 \%$ \\
\hline 5 & 1.32 & 0.31 & $6.1 \%$ & 0.02 & $0.4 \%$ \\
\hline 10 & 2.64 & 1.14 & $11.4 \%$ & 0.07 & $0.7 \%$ \\
\hline 20 & 5.28 & 4.38 & $21.9 \%$ & 0.27 & $1.4 \%$ \\
\hline 15 & 3.96 & 2.50 & $16.7 \%$ & 0.16 & $1.0 \%$ \\
\hline
\end{tabular}

dimensional Time, $\mathrm{t}$ Time, $\mathrm{t}$ Time, $\mathrm{t}$ Time, $\mathrm{t}$

\begin{tabular}{cccccc} 
time, $\mathrm{t}_{\mathrm{D}}$ & $(\mathrm{d})$ & $(\mathrm{hr})$ & $(\mathrm{min})$ & $(\mathrm{s})$ & \\
\cline { 1 - 5 } $4.0 \mathrm{E}+02$ & 0.0 & 0.1 & 7.5 & 448.0 & $10: 1$ \\
4.0E+03 & 0.1 & 1.2 & 74.7 & 4479.5 & $100: 1$ \\
4.0E+04 & 0.5 & 12.4 & 746.6 & 44795.2 & $\mathrm{r} / \mathrm{b}<0.125$ \\
$1.0 \mathrm{E}+06$ & 13.0 & 311.1 & 18664.7 & $\# \# \# \# \# \#$ & long time
\end{tabular}


Table $7 \quad$ Field data for SWP-300A.

\begin{tabular}{|c|c|c|c|c|c|c|c|c|c|c|c|c|c|c|c|c|}
\hline $\begin{array}{c}\text { Well ID } \\
\text { SWP-300A }\end{array}$ & $\begin{array}{c}\text { Top of } \\
\text { Screen } \\
\text { From } \\
\text { Grade (tt) } \\
\\
162.19 \\
\end{array}$ & 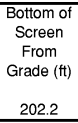 & $\begin{array}{c}\begin{array}{c}\text { Screen } \\
\text { Length (tt) }\end{array} \\
40.01 \\
\end{array}$ & $\begin{array}{c}\begin{array}{c}\text { Casing } \\
\text { Diameter } \\
\text { (inch) }\end{array} \\
4 \\
\end{array}$ & $\begin{array}{c}\text { Approx. } \\
\text { Water Level } \\
\text { From TOC } \\
\text { (tt) } \\
114.75\end{array}$ & 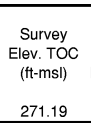 & $\begin{array}{c}\text { Survey } \\
\text { Elev. Conc } \\
\text { Pad (tt-msl) } \\
268.69\end{array}$ & $\begin{array}{l}\text { TOC to Conc } \\
\text { Pad (ft) } \\
2.5\end{array}$ & $\begin{array}{c}\text { Pad } \\
\text { Thickness } \\
\text { (ft) } \\
0.33\end{array}$ & $\begin{array}{l}\text { Top of } \\
\text { Screen From } \\
\text { TOC (ft) } \\
165.02\end{array}$ & 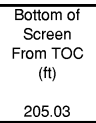 & $\begin{array}{c}\text { Top of } \\
\text { Screen } \\
\text { Elevation } \\
\text { (tt-msl) } \\
106.17 \\
\end{array}$ & 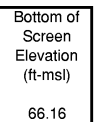 & & & \\
\hline & \begin{tabular}{|l|} 
Probe \\
Date
\end{tabular} & \multicolumn{2}{|c|}{$\begin{array}{c}.5^{\prime \prime} \\
7 / 24 / 00\end{array}$} & \multicolumn{2}{|c|}{$\begin{array}{c}1.0 " \\
7 / 25 / 00\end{array}$} & \multicolumn{3}{|c|}{$\begin{array}{c}1.0 " \\
7 / 25 / 00\end{array}$} & $\begin{array}{c}5^{n} \\
3 / 29 / 00 \\
\end{array}$ & $\begin{array}{c}1.0 " 10 \\
3 / 31 / 00 \\
\end{array}$ & & \multicolumn{5}{|c|}{$7 / 24 / 00$} \\
\hline Station & $\begin{array}{c}\text { Depth } \\
\text { Below } \\
\text { TOC (ft) } \\
\end{array}$ & Time & $\begin{array}{c}\text { Ambient } \\
\text { Instrument } \\
\text { Response } \\
\text { (L/min) } \\
\end{array}$ & Time & $\begin{array}{c}\text { Dynamic } \\
\text { Flow } \\
\text { Instrument } \\
\text { Response } \\
\text { (L/min) } \\
\end{array}$ & \begin{tabular}{|c|}
$\begin{array}{c}\text { Delta Time } \\
\min \end{array}$ \\
\end{tabular} & Delta (ml) & \begin{tabular}{|c|} 
Dynamic \\
Instrument \\
Response \\
Corrected for \\
"0" \\
\end{tabular} & $\begin{array}{c}y=0.0024 \times 2 \\
+0.7838 x \\
\text { Ambient } \\
\text { Calibrated } \\
\text { Flow (L/min) }\end{array}$ & \begin{tabular}{|c|}
$y=0.9797 x$ \\
+0.1097 \\
Dynamic \\
Calibrated \\
Flow $(\mathrm{L} / \mathrm{min})$ \\
\end{tabular} & & Time & $\begin{array}{c}\text { Water Level } \\
\text { (ft) }\end{array}$ & $\begin{array}{l}\text { Time to } \\
\text { Fill } \\
\text { Bucket } \\
16.8 \mathrm{~L} \\
(\mathrm{sec}) \\
\end{array}$ & $\begin{array}{c}\text { Field } \\
\text { Measured } \\
\text { Flow } \\
\text { (L/min) } \\
\end{array}$ & $\begin{array}{c}\text { Panametric } \\
\text { Flow } \\
\text { Reading Ref } \\
\text { Only (L/min) } \\
\end{array}$ \\
\hline 48 & 157.0 & 14:10 & -0.002 & 11.52 & 1357 & 105 & 0000 & 1359 & -0.0016 & 1340 & & 14:15 & 53.2 & $N / A$ & $N / A$ & $N / A$ \\
\hline 41 & $\begin{array}{l}164.0 \\
164.0\end{array}$ & $\begin{array}{l}14.06 \\
14: 02\end{array}$ & $\begin{array}{l}-0.004 \\
-0.004\end{array}$ & $\begin{array}{l}11.52 \\
11: 49\end{array}$ & $\begin{array}{l}13.57 \\
13.57\end{array}$ & 102 & 0.0194 & $\begin{array}{l}13.59 \\
13.59\end{array}$ & $\begin{array}{l}-0.0031 \\
-0.0031\end{array}$ & $\begin{array}{l}13.42 \\
13.42\end{array}$ & bypass ratio & & & & & \\
\hline 40 & 165.0 & $14: 00$ & -0.004 & $11: 46$ & 13.48 & 99 & 0.0189 & 13.50 & -0.0031 & 13.33 & 0.681 & & & & & \\
\hline 39 & 166.0 & 13:58 & 0.025 & $11: 42$ & 9.14 & 95 & 0.0181 & 9.16 & 0.0196 & 9.08 & & & & $7 / 25 / 00$ & & \\
\hline 38 & 167.0 & 13:55 & 0.021 & 11:39 & 9.21 & 92 & 0.0175 & 9.23 & 0.0165 & 9.15 & & & & & & \\
\hline 37 & 168.0 & 13:53 & 0.021 & 11:36 & 9.24 & 89 & 0.0170 & 9.26 & 0.0165 & 9.18 & & 9:37 & See Data & 76 & 13.26 & \\
\hline 36 & 169.0 & $13: 50$ & 0.022 & $11: 33$ & 9.20 & 86 & 0.0164 & 9.22 & 0.0172 & 9.14 & & 9:42 & Logger & 76 & 13.26 & \\
\hline 35 & 170.0 & 13:48 & 0.023 & 11:31 & 9.22 & 84 & 0.0160 & 9.24 & 0.0180 & 9.16 & & 9:59 & & 76 & 13.26 & \\
\hline 34 & 171.0 & 13:46 & 0.016 & $11: 28$ & 9.22 & 81 & 0.0154 & 9.24 & 0.0125 & 9.16 & & $10: 02$ & & & & 13.21 \\
\hline 33 & 172.0 & 13:44 & 0.016 & $11: 24$ & 9.11 & 77 & 0.0147 & 9.12 & 0.0125 & 9.05 & & 10:17 & & & & 13.29 \\
\hline 32 & 173.0 & 13:42 & 0.015 & $11: 22$ & 9.12 & 75 & 0.0143 & 9.13 & 0.0118 & 9.06 & & $10: 18$ & & 76 & 13.26 & \\
\hline 31 & 1740 & $13 \cdot 38$ & 0.009 & $11 \cdot 19$ & 920 & 72 & 0.0137 & 921 & 0.0071 & 9.14 & bypass ratio & $10 \cdot 23$ & & & & 13.10 \\
\hline 30 & 175.0 & 13:35 & -0.093 & 11:17 & 13.10 & 70 & 0.0133 & 13.11 & -0.0729 & 12.96 & 0.698 & $10: 25$ & & & & 13.21 \\
\hline 29 & 176.0 & $13: 33$ & 0.011 & $11: 13$ & 9.02 & 66 & 0.0126 & 9.03 & 0.0086 & 8.96 & & $10: 27$ & & & & 13.25 \\
\hline 28 & 177.0 & $13: 29$ & 0.006 & 11:11 & 8.87 & 64 & 0.0122 & 8.88 & 0.0047 & 8.81 & & 10:29 & & & & 13.28 \\
\hline 27 & 178.0 & 13:22 & 0.000 & $11: 08$ & 8.84 & 61 & 0.0116 & 8.85 & 0.0000 & 8.78 & & $10: 32$ & & 76 & 13.26 & 13.22 \\
\hline 26 & 179.0 & 13:15 & -0.100 & 11:06 & 8.74 & 59 & 0.0112 & 8.75 & -0.0784 & 8.68 & & $10: 39$ & & & & 13.29 \\
\hline 25 & 180.0 & $13: 13$ & -0.115 & $11: 04$ & 8.78 & 57 & 0.0109 & 8.79 & -0.0901 & 8.72 & & $10: 44$ & & & & 13.34 \\
\hline 24 & 181.0 & 13:11 & -0.125 & $11: 02$ & 8.71 & 55 & 0.0105 & 8.72 & $\begin{array}{l}-0.0979 \\
\end{array}$ & 8.65 & & $10: 54$ & & & & 13.28 \\
\hline 23 & 182.0 & 13:09 & -0.130 & $10: 59$ & 8.72 & 52 & 0.0099 & 8.73 & -0.1019 & 8.66 & & $10: 56$ & & & & 13.34 \\
\hline 22 & 183.0 & 13:07 & -0.136 & $10: 57$ & 8.75 & 50 & 0.0095 & 8.76 & -0.1066 & 8.69 & & $11: 01$ & & 75 & 13.44 & 13.36 \\
\hline $\begin{array}{l}21 \\
21\end{array}$ & $\begin{array}{l}184.0 \\
184.0\end{array}$ & $\begin{array}{l}13: 05 \\
1305\end{array}$ & $\begin{array}{l}-0.140 \\
-0.142\end{array}$ & $10: 55$ & 8.66 & 48 & 0.0091 & 8.67 & $\begin{array}{l}-0.1113 \\
-0.11300\end{array}$ & $\begin{array}{l}8.09 \\
8.60\end{array}$ & bypass ratio & $\begin{array}{l}11: 07 \\
\end{array}$ & & & & $\begin{array}{l}13.30 \\
13.30\end{array}$ \\
\hline 20 & 185.0 & 13:03 & -0.262 & $10: 53$ & 12.08 & 46 & 0.0088 & 12.09 & -0.2052 & 11.95 & 0.719 & 11:10 & & & & 13.42 \\
\hline 19 & 186.0 & 13:00 & -0.178 & $10: 50$ & 8.64 & 43 & 0.0082 & 8.65 & -0.1394 & 8.58 & & 11:19 & & 75 & 13.44 & \\
\hline 18 & 187.0 & 12:58 & -0.179 & $10: 48$ & 8.52 & 41 & 0.0078 & 8.53 & -0.1402 & 8.46 & & $11: 28$ & & & & 13.34 \\
\hline 17 & 188.0 & $12: 55$ & -0.195 & $10: 46$ & 8.43 & 39 & 0.0074 & 8.44 & -0.1527 & 8.38 & & $11: 32$ & & & & 13.51 \\
\hline 16 & 189.0 & 12:50 & -0.195 & $10: 44$ & 8.32 & 37 & 0.0070 & 8.33 & -0.1527 & 8.27 & & $11: 35$ & & & & 13.51 \\
\hline 15 & 190.0 & $12: 48$ & -0.210 & 10:42 & 8.34 & 35 & 0.0067 & 8.35 & -0.1645 & 8.29 & & $11: 37$ & & 75 & 13.44 & \\
\hline 14 & 191.0 & $12: 47$ & -0.210 & $10: 40$ & 8.11 & 33 & 0.0063 & 8.12 & -0.1645 & 8.0 & & $11: 40$ & & & & 13.44 \\
\hline 13 & 192.0 & 12:45 & -0.243 & $10: 38$ & 7.09 & 31 & 0.0059 & 7.10 & -0.1903 & 7.06 & & $11: 47$ & & & & 13.43 \\
\hline 12 & 193.0 & 12:44 & -0.265 & $10: 33$ & 6.01 & 26 & 0.0050 & 6.01 & -0.2075 & 6.00 & & $11: 50$ & & & & 13.46 \\
\hline 11 & 194.0 & 12:42 & -0.262 & $10: 31$ & 5.85 & 24 & 0.0046 & 5.85 & -0.2052 & 5.85 & bypass ratio & $11: 53$ & & 73 & 13.81 & \\
\hline 10 & 195.0 & $12: 40$ & -0.444 & $10: 28$ & 7.78 & 21 & 0.0040 & 7.78 & -0.3475 & 7.74 & 0.756 & & & & & \\
\hline 9 & 196.0 & 12:39 & -0.280 & $10: 26$ & 5.85 & 19 & 0.0036 & 5.85 & -0.2193 & 5.84 & & & & & & \\
\hline 8 & 197.0 & $12: 37$ & -0.287 & $10: 24$ & 5.74 & 17 & 0.0032 & 5.74 & -0.2248 & 5.74 & & & & & & \\
\hline 7 & 198.0 & $12: 35$ & -0.266 & $10: 22$ & 5.44 & 15 & 0.0029 & 5.44 & -0.2083 & 5.4 & & & & & & \\
\hline 6 & 199.0 & $12: 33$ & -0.255 & $10: 20$ & 5.15 & 13 & 0.0025 & 5.15 & -0.1997 & 5.16 & & & & & & \\
\hline 5 & 200.0 & $12: 32$ & -0.237 & 10:16 & 5.00 & 9 & 0.0017 & 5.00 & -0.1856 & 5.01 & & & & & & \\
\hline 4 & 201.0 & $12: 30$ & -0.176 & 10:11 & 3.73 & 4 & 0.0008 & 3.73 & -0.1379 & 3.76 & & & & & & \\
\hline $\begin{array}{l}3 \\
2\end{array}$ & 202.3 & $12: 28$ & 0.000 & $10: 07$ & $\begin{array}{l}1.02 \\
\text { S }\end{array}$ & 0 & 0.0000 & 1.02 & 0.0000 & 1.11 & & & & & & \\
\hline 1 & & & & & & & & & & & & & & & & \\
\hline
\end{tabular}

Note: Meter at bottom of well at 202.3'

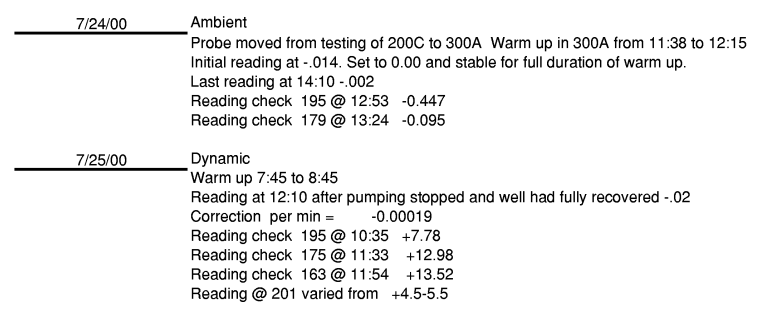




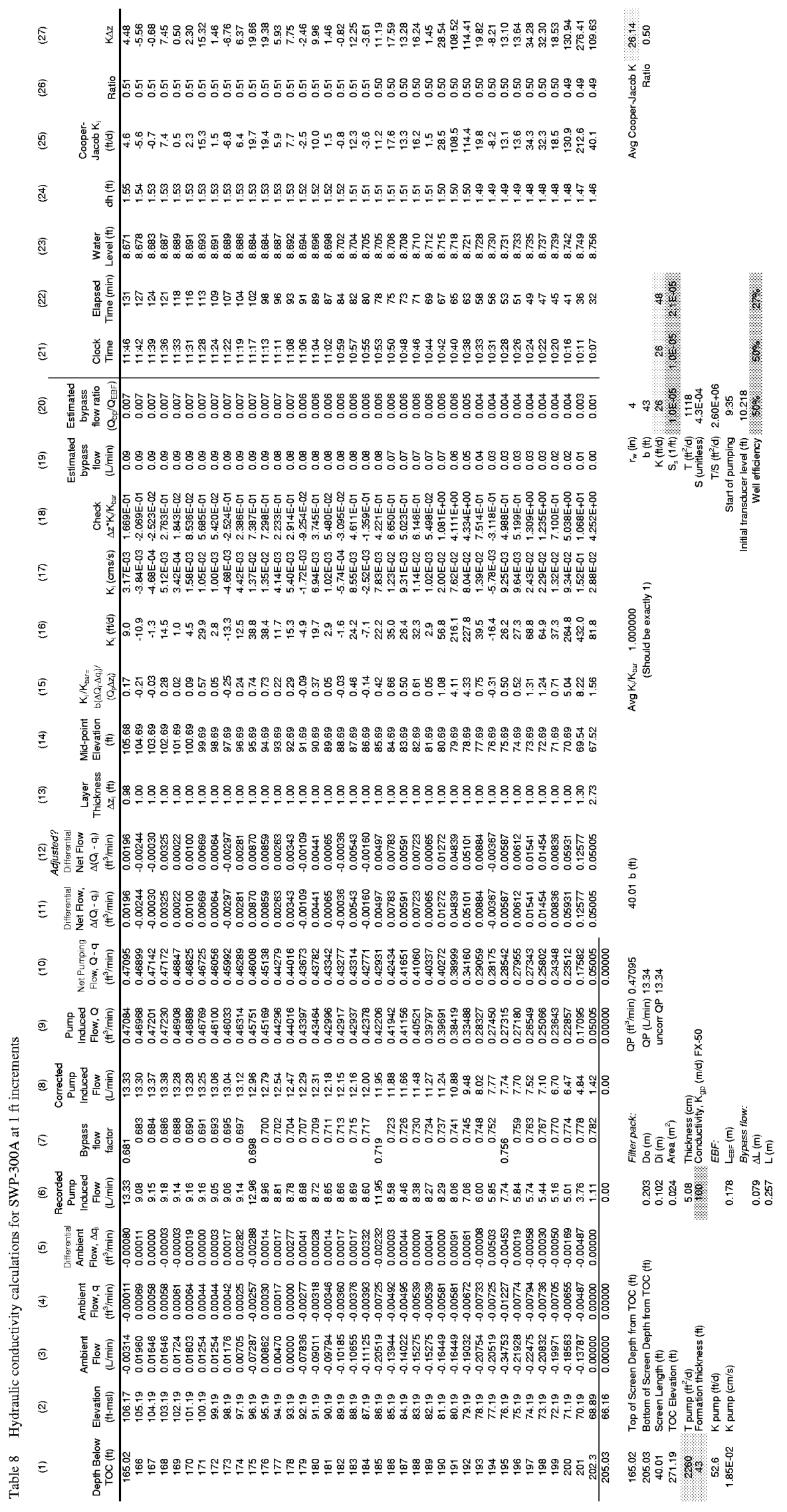




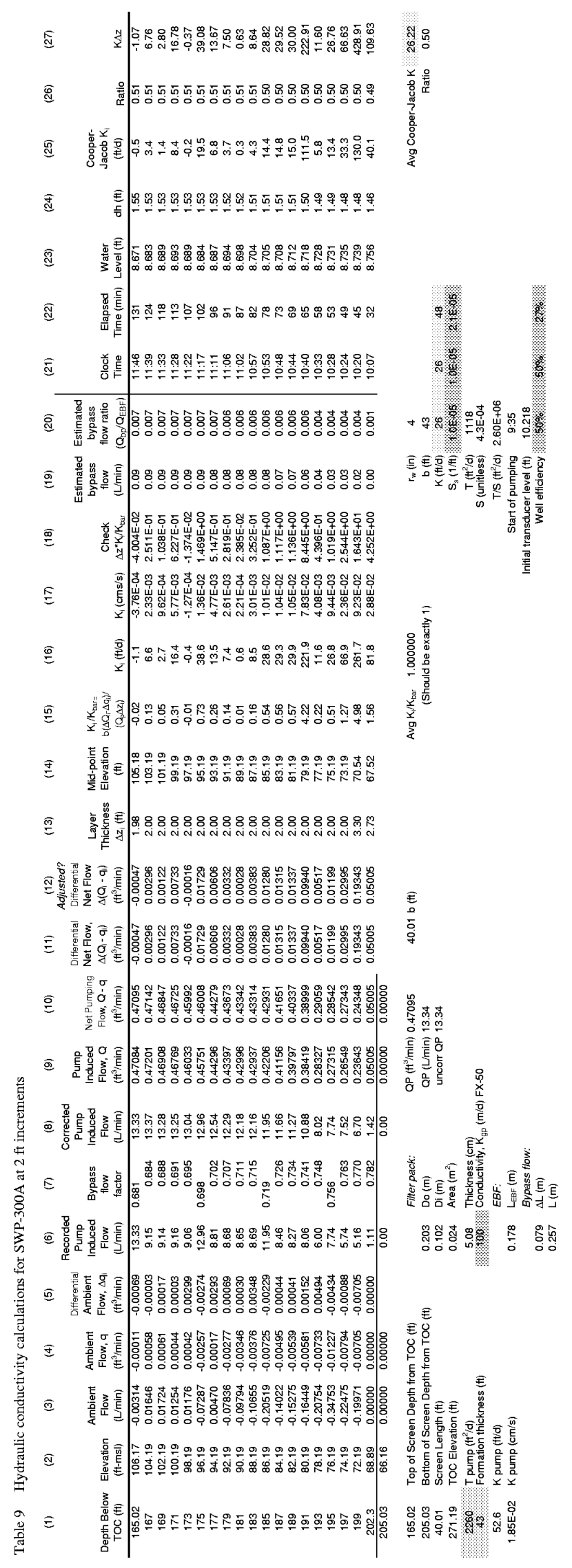




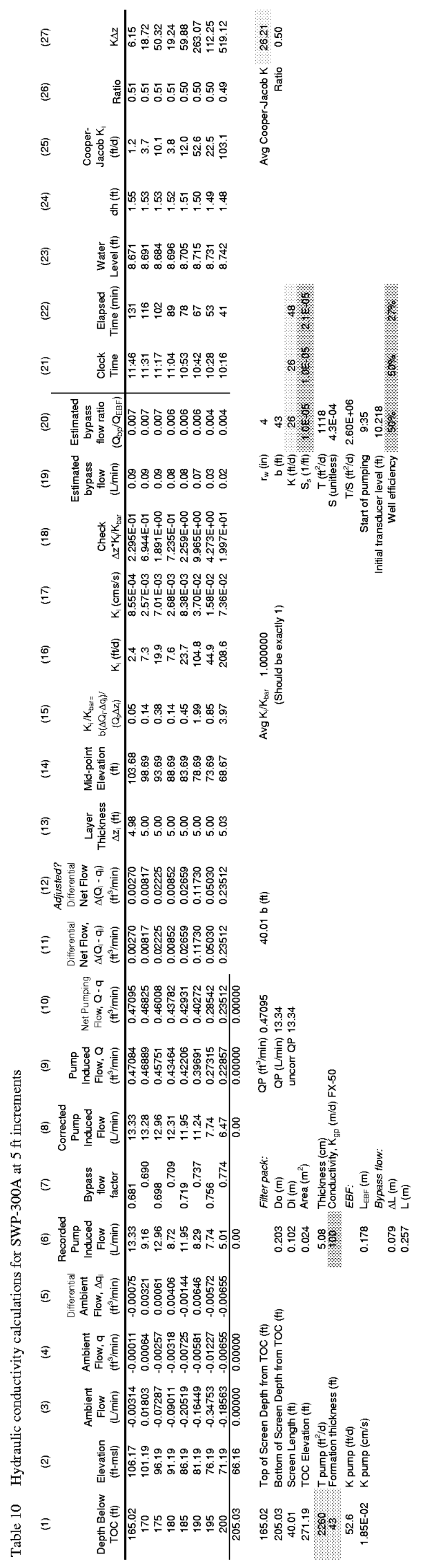




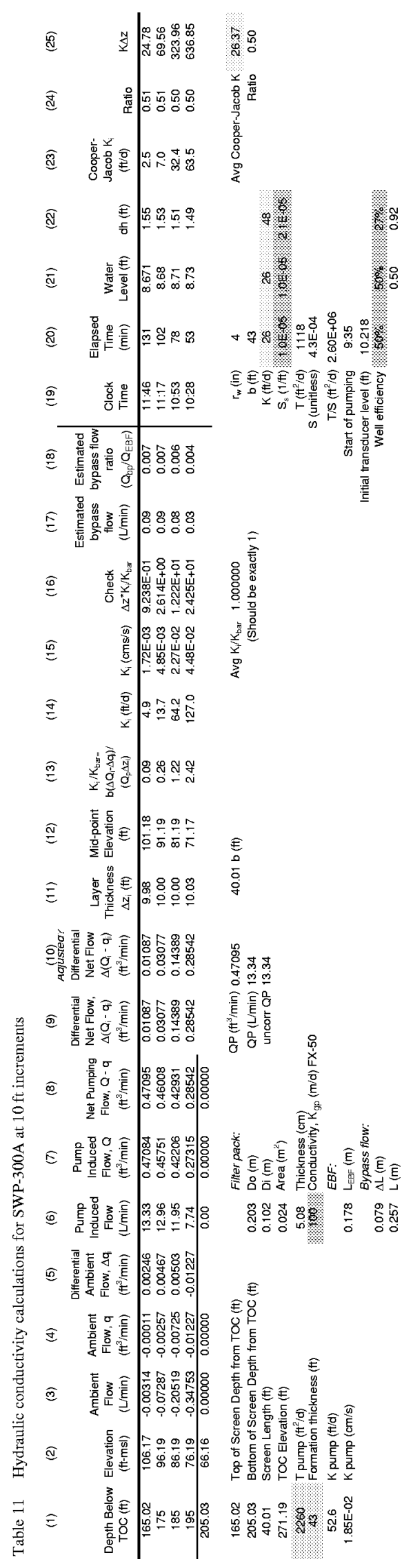


Table 12 Field data for SWP-200C.

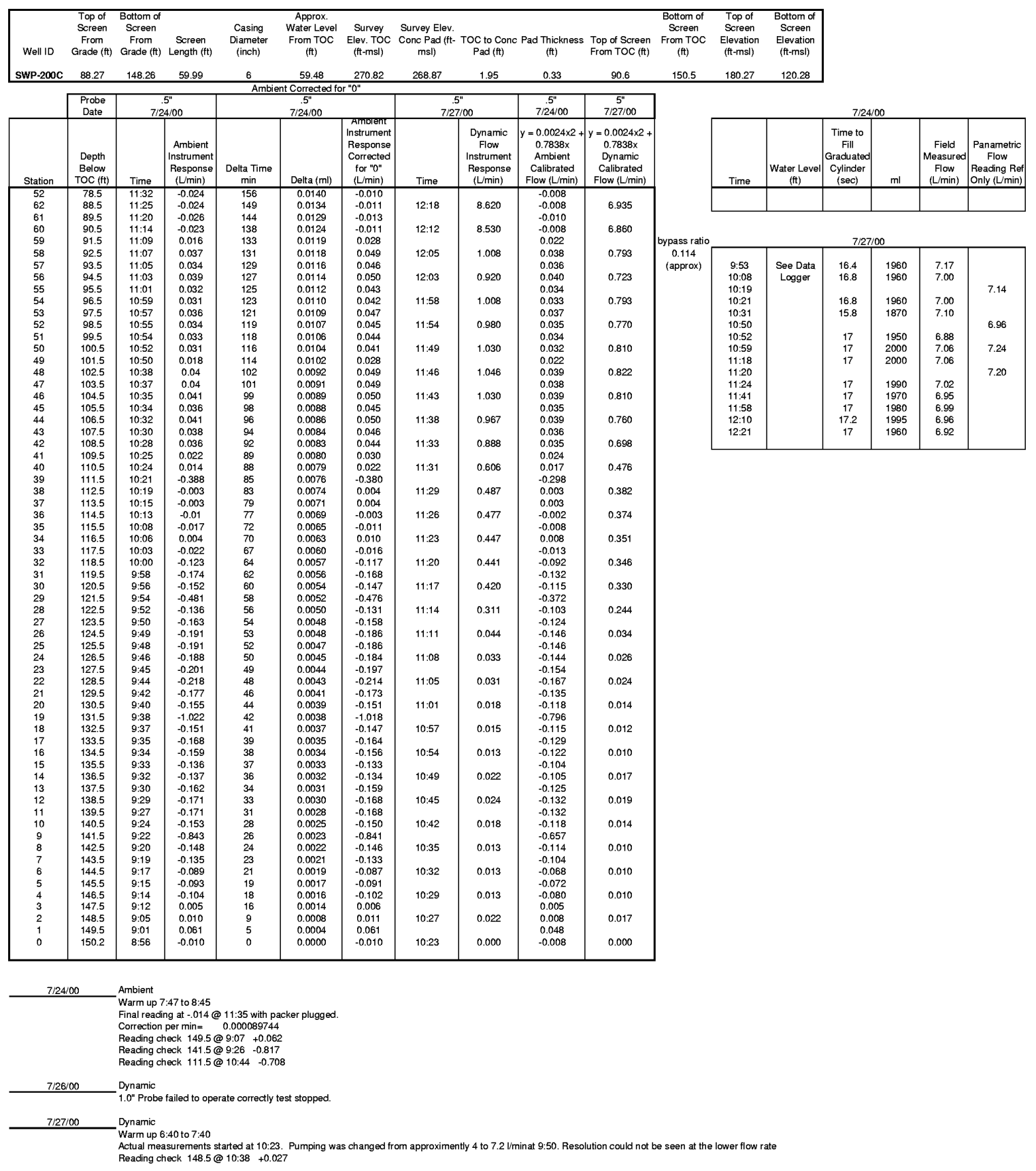




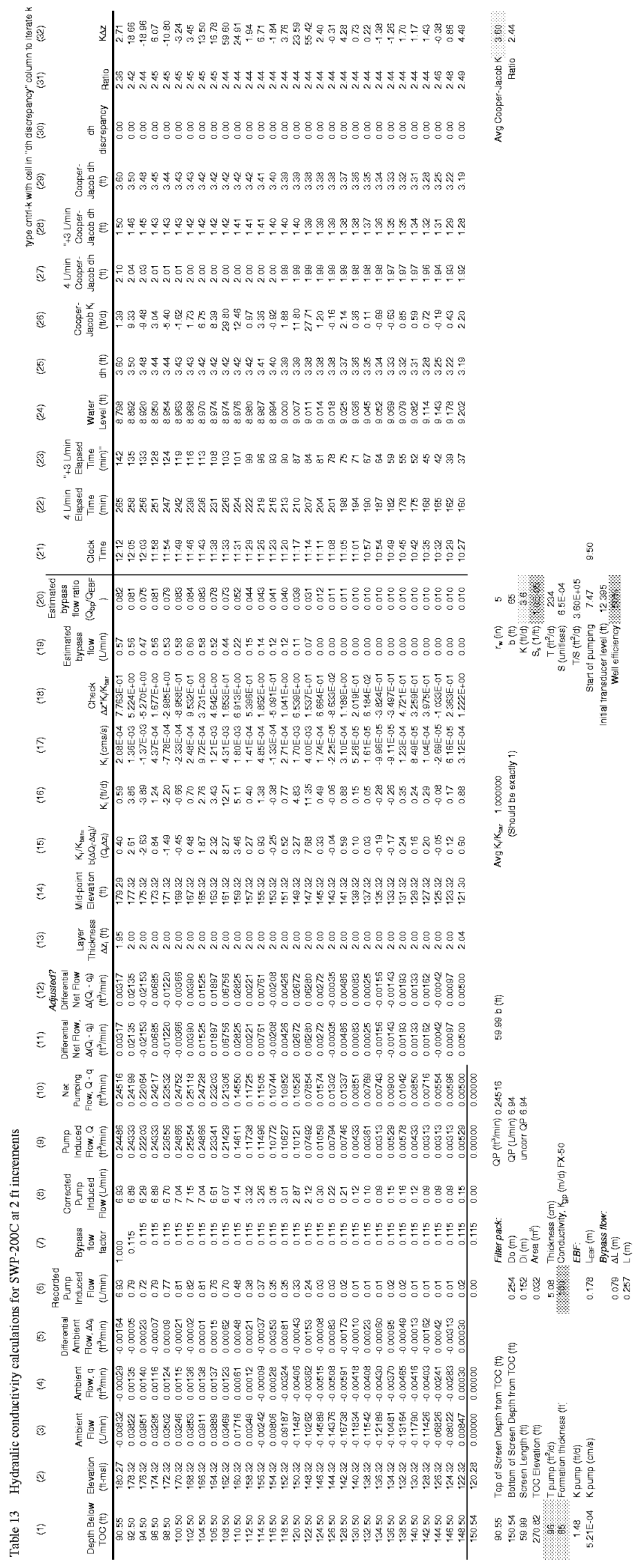




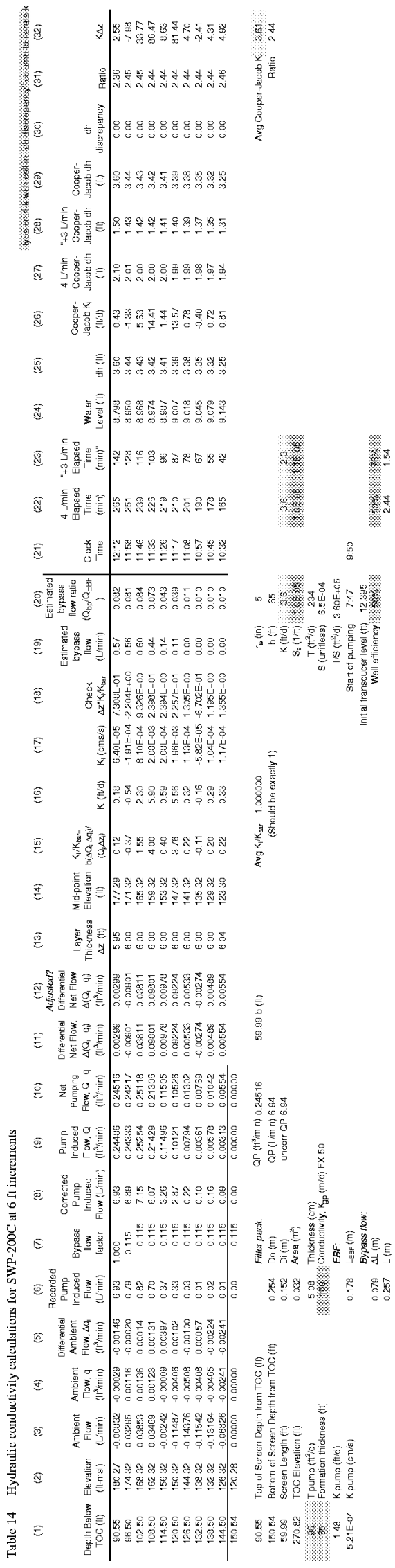


Table 15 Field data for SWP-100D.

\begin{tabular}{|c|c|c|c|c|c|c|c|c|c|c|c|c|c|}
\hline Well ID & $\begin{array}{c}\text { Top of } \\
\text { Screen } \\
\text { From } \\
\text { Grade (ft) }\end{array}$ & $\begin{array}{c}\text { Bottom of } \\
\text { Screen } \\
\text { From } \\
\text { Grade (ft) }\end{array}$ & $\begin{array}{c}\text { Screen } \\
\text { Length (ft) }\end{array}$ & $\begin{array}{l}\text { Casing } \\
\text { Diameter } \\
\text { (inch) }\end{array}$ & $\begin{array}{c}\text { Approx. } \\
\text { Water Level } \\
\text { From TOC } \\
\text { (ft) }\end{array}$ & $\begin{array}{l}\text { Survey Elev. } \\
\text { TOC (ft-msl) }\end{array}$ & $\begin{array}{l}\text { Survey } \\
\text { Elev. Conc } \\
\text { Pad (ft-msl) }\end{array}$ & $\begin{array}{l}\text { TOC to } \\
\text { Conc Pad } \\
\text { (ft) }\end{array}$ & $\begin{array}{c}\text { Pad } \\
\text { Thickness } \\
\text { (ft) }\end{array}$ & $\begin{array}{c}\text { Top of } \\
\text { Screen } \\
\text { From TOC } \\
\text { (ft) }\end{array}$ & $\begin{array}{c}\text { Bottom of } \\
\text { Screen } \\
\text { From TOC } \\
\text { (ft) }\end{array}$ & $\begin{array}{c}\text { Top of } \\
\text { Screen } \\
\text { Elevation } \\
\text { (ft) }\end{array}$ & $\begin{array}{c}\text { Bottom of } \\
\text { Screen } \\
\text { Elevation } \\
(\mathrm{ft})\end{array}$ \\
\hline WP-100D & 45.18 & 75.14 & 29.96 & 6 & 53.45 & 270.77 & 268.41 & 2.36 & 0.33 & 47.87 & 77.8 & 222.90 & 192.94 \\
\hline
\end{tabular}

\begin{tabular}{|c|c|c|c|c|c|c|c|c|c|c|c|c|c|}
\hline \multirow[b]{2}{*}{ Station } & \begin{tabular}{|l} 
Probe \\
Date \\
\end{tabular} & \multicolumn{2}{|c|}{$\begin{array}{c}.5 " \\
7 / 24 / 00 \\
\end{array}$} & \multicolumn{2}{|c|}{$\begin{array}{c}1.0^{\prime \prime} \\
7 / 25 / 00 \\
\end{array}$} & $\begin{array}{c}.5 " \\
7 / 24 / 00 \\
\end{array}$ & $\begin{array}{c}1.0^{\prime \prime} \\
7 / 25 / 00 \\
\end{array}$ & & \multicolumn{5}{|c|}{$7 / 24 / 00$} \\
\hline & $\begin{array}{c}\text { Depth } \\
\text { Below } \\
\text { TOC (ft) } \\
\end{array}$ & Time & $\begin{array}{c}\text { Ambient } \\
\text { Instrument } \\
\text { Response } \\
(\mathrm{L} / \mathrm{min})\end{array}$ & Time & $\begin{array}{c}\text { Dynamic } \\
\text { Instrument } \\
\text { Response } \\
\text { (L/min) }\end{array}$ & $\begin{array}{c}\mathrm{y}=0.0024 \times 2+ \\
0.7838 \mathrm{x} \\
\text { Ambient } \\
\text { Calibrated } \\
\text { Flow }(\Delta \mathrm{min}) \\
\end{array}$ & $\begin{array}{c}y=0.9797 x \\
+0.1097 \\
\text { Dynamic } \\
\text { Calibrated } \\
\text { Flow (L/min) }\end{array}$ & & Time & $\begin{array}{c}\text { Water } \\
\text { Level (tt) }\end{array}$ & $\begin{array}{c}\text { Time to } \\
\text { Fill } \\
\text { Bucket } \\
16.8 \mathrm{~L} \\
\text { (sec) } \\
\end{array}$ & $\begin{array}{c}\text { Field } \\
\text { Measured } \\
\text { Flow } \\
(\llcorner/ \mathrm{min}) \\
\end{array}$ & \begin{tabular}{|c|} 
Panametri \\
c Flow \\
Reading \\
Ref Only \\
$(\llcorner/$ min $)$ \\
\end{tabular} \\
\hline $\begin{array}{l}31 \\
30 \\
29\end{array}$ & $\begin{array}{l}46.8 \\
47.8 \\
48.8\end{array}$ & & & & & & & & $14: 15$ & 53.2 & N/A & $\mathrm{N} / \mathrm{A}$ & $\mathrm{N} / \mathrm{A}$ \\
\hline $\begin{array}{l}28 \\
27 \\
26\end{array}$ & $\begin{array}{l}49.8 \\
50.8 \\
51.8\end{array}$ & & & & & & & & & & $7 / 25 / 00$ & & \\
\hline 25 & 52.8 & & & & & & & & & & & & \\
\hline 24 & 53.8 & $15: 54$ & 0.056 & & & 0.044 & & & 14:05 & See Data & 134 & 7.52 & \\
\hline 23 & 54.8 & $15: 52$ & 0.056 & & & 0.044 & & & $14: 06$ & Logger & & & 7.57 \\
\hline 22 & 55.8 & $15: 50$ & 0.052 & & & 0.041 & & & $14: 20$ & & & & 7.54 \\
\hline 21 & 56.8 & $15: 48$ & 0.056 & & & 0.044 & & & $14: 25$ & & 136 & 7.41 & 7.57 \\
\hline 20 & 57.8 & $15: 46$ & -0.005 & & & -0.004 & & & $14: 45$ & & 136 & 7.41 & 7.49 \\
\hline 19 & 58.8 & $15: 40$ & 0.052 & & & 0.041 & & & 15:07 & & & & 7.53 \\
\hline 18 & 59.8 & $15: 38$ & 0.053 & $16: 27$ & 3.20 & 0.042 & 3.24 & & $15: 11$ & & 140 & 7.20 & \\
\hline 17 & 60.8 & $15: 36$ & 0.050 & $16: 15$ & 3.42 & 0.039 & 3.46 & & $15: 28$ & & & & 7.47 \\
\hline 16 & 61.8 & $15: 35$ & 0.054 & $16: 06$ & 3.14 & 0.042 & 3.19 & & $15: 42$ & & & & 7.5 \\
\hline 15 & 62.8 & $15: 33$ & 0.054 & $16: 04$ & 2.97 & 0.042 & 3.02 & & $15: 45$ & & 137 & 7.36 & \\
\hline 14 & 63.8 & $15: 31$ & 0.052 & $16: 01$ & 3.10 & 0.041 & 3.15 & & $15: 57$ & & & & 7.45 \\
\hline 13 & 64.8 & $15: 24$ & 0.047 & $15: 58$ & 3.12 & 0.037 & 3.17 & & $16: 04$ & & & & 7.43 \\
\hline 12 & 65.8 & $15: 21$ & 0.052 & $15: 52$ & 2.94 & 0.041 & 2.99 & & $16: 07$ & & 137 & 7.36 & \\
\hline 11 & 66.8 & $15: 19$ & 0.047 & $15: 49$ & 2.66 & 0.037 & 2.72 & bypass ratio & $16: 30$ & & & & 7.45 \\
\hline 10 & 67.8 & $15: 16$ & -0.018 & $15: 47$ & 5.42 & -0.014 & 5.42 & 0.483 & & & & average & 7.50 \\
\hline 9 & 68.8 & $15: 12$ & 0.050 & $15: 40$ & 2.46 & 0.039 & 2.52 & & & & & & \\
\hline 8 & 69.8 & $15: 10$ & 0.046 & $15: 36$ & 2.24 & 0.036 & 2.30 & & & & & & \\
\hline 7 & 70.8 & $15: 09$ & 0.044 & $15: 33$ & 2.13 & 0.034 & 2.20 & & & & & & \\
\hline 6 & 71.8 & $15: 07$ & 0.044 & $15: 31$ & 2.09 & 0.034 & 2.16 & & & & & & \\
\hline 5 & 72.8 & 15:06 & 0.043 & $15: 29$ & 1.90 & 0.034 & 1.97 & & & & & & \\
\hline 4 & 73.8 & 15:05 & 0.047 & $15: 26$ & 1.56 & 0.037 & 1.64 & & & & & & \\
\hline 3 & 74.8 & $15: 04$ & 0.045 & $15: 24$ & 1.39 & 0.035 & 1.47 & & & & & & \\
\hline 2 & 75.8 & $15: 02$ & 0.042 & $15: 21$ & 0.95 & 0.033 & 1.04 & & & & & & \\
\hline 1 & 76.8 & $15: 00$ & 0.017 & $15: 18$ & 0.66 & 0.013 & 0.76 & & & & & & \\
\hline 0 & 77.8 & $14: 57$ & 0.000 & 15:09 & 0.00 & 0.000 & 0.11 & & & & & & \\
\hline
\end{tabular}

Correction tor " 0 "

Start Tirne - Stop Time $-180 \mathrm{~min}$

otal Detta $0^{\prime \prime}=032$

Delta/min $=0321180=\quad 0,000178$

7/24/00 Ambient

Probe moved from testing of 300A to 100D Warm up in 100D from 14:17 to 14:47.

Initial reading at -.005 . Set to 0.00 and stable for full duration of warm up.

Reading check $67.8 @ 15: 29-0.010$

Reading check $77.8 @ 15: 57 \quad 0.000$

7/25/00 Dynamic

Warm up $14: 00$ to $14: 58$

16:17 Tranducer raised up 2

Reading check 67.8@ 15:55 +5.36

Reading check 62.8@ 16:11+3.20 


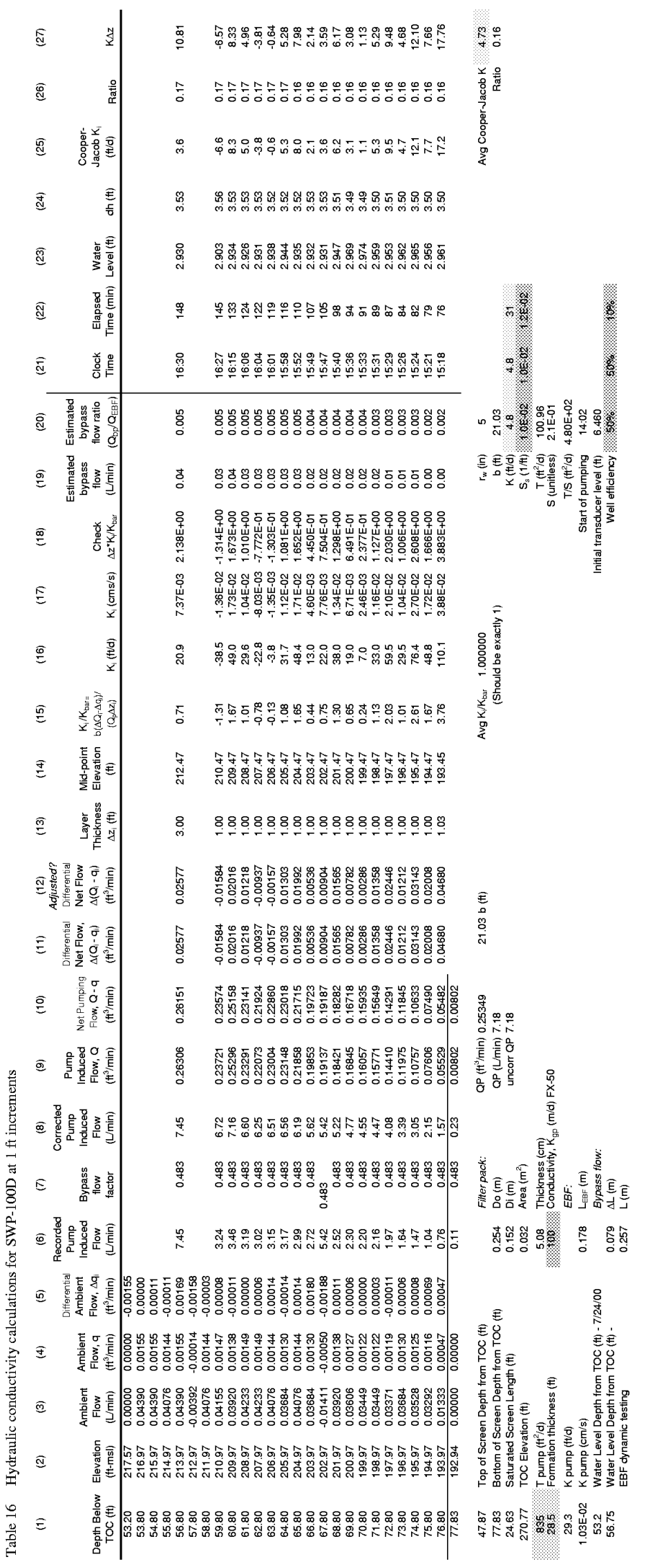




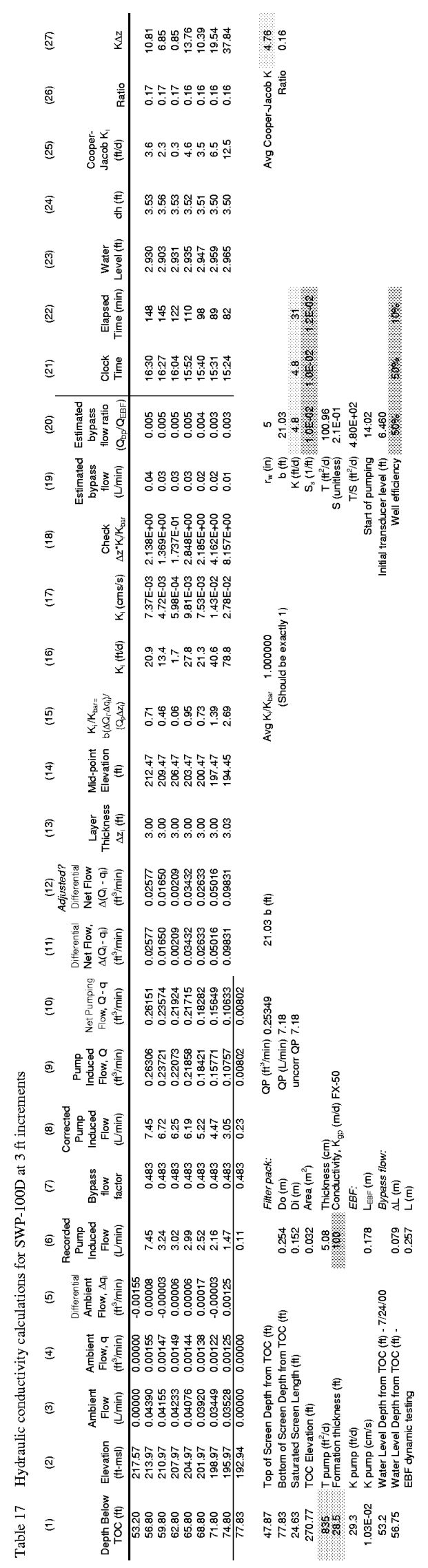




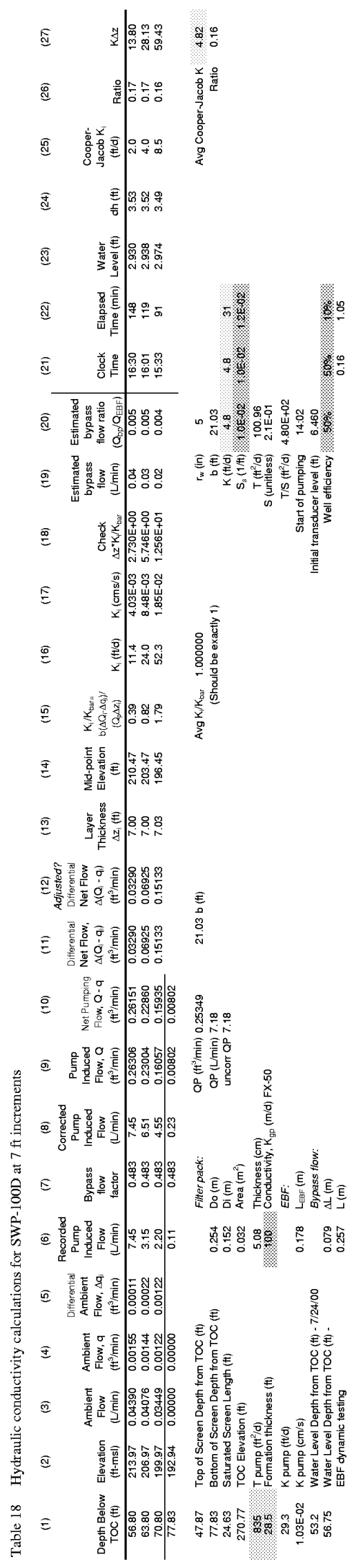


Table 19 Comparison of average conductivity estimates.

\begin{tabular}{|l|c|c|c|}
\hline \multirow{2}{*}{ Aquifer test } & \multicolumn{2}{c|}{ Horizontal conductivity (ft/d) } \\
\cline { 2 - 4 } & $\begin{array}{c}\text { Gordon } \\
\text { SWP-300A }\end{array}$ & $\begin{array}{c}\text { lower UTRA } \\
\text { SWP-200C }\end{array}$ & $\begin{array}{c}\text { upper UTRA } \\
\text { SWP-100D }\end{array}$ \\
\hline $\begin{array}{l}\text { Multiple-well } \\
\text { (WSRC, 1999) }\end{array}$ & 52.6 & 1.48 & 29.3 \\
\hline Single-well concurrent with EBF testing & 23 & 2.3 & 12 \\
\hline $\begin{array}{l}\text { Cooper-Jacob analysis applied to } \\
\text { individual screen intervals - } \\
\text { assumed well efficiency and specific } \\
\text { storage }\end{array}$ & 26 & 3.6 & 4.8 \\
\hline $\begin{array}{l}\text { Cooper-Jacob analysis applied to } \\
\text { individual screen intervals - } \\
\text { well efficiency and specific storage from } \\
\text { WSRC (1999) }\end{array}$ & 48 & 2.3 & 31 \\
\hline
\end{tabular}




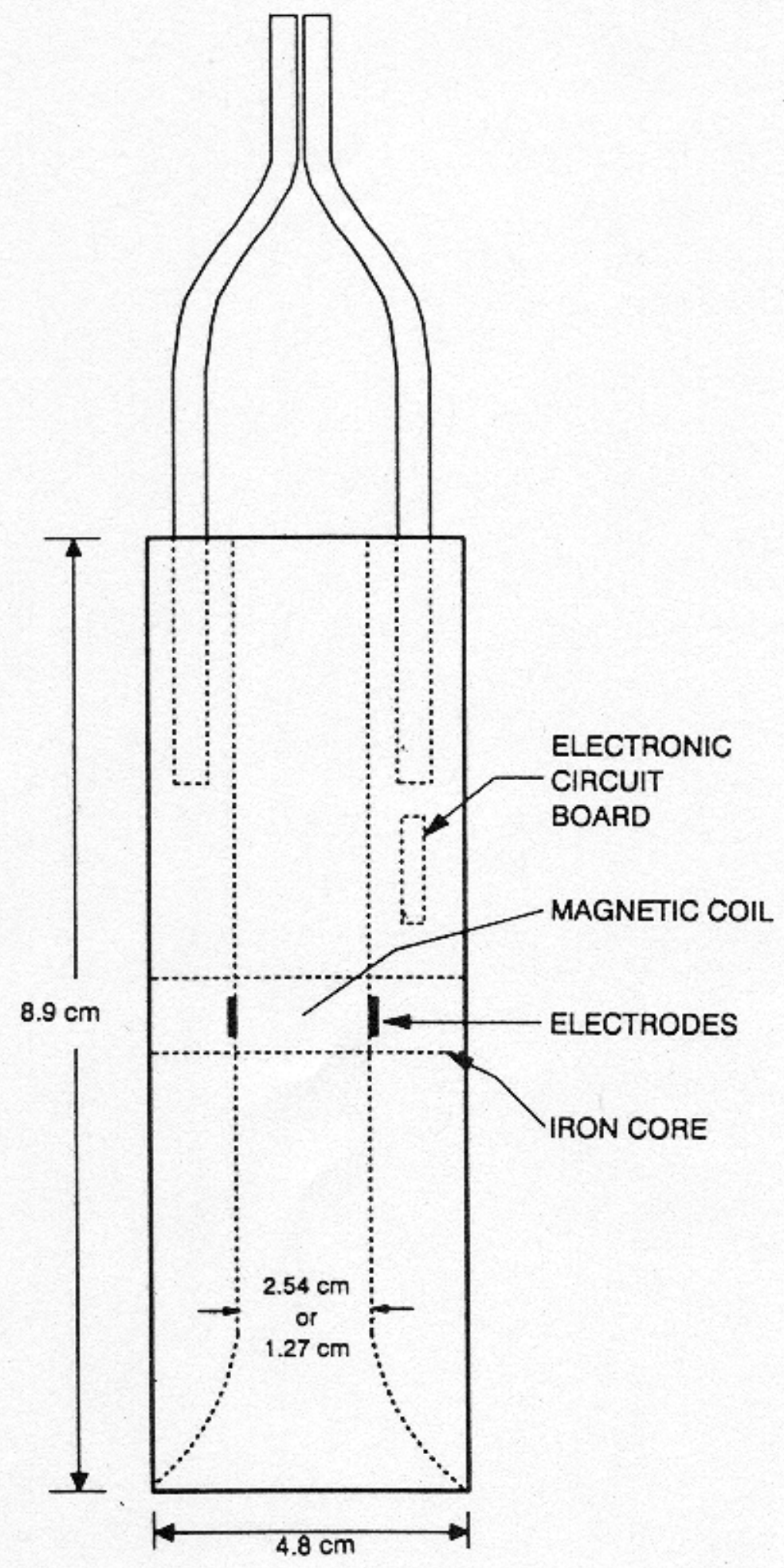

Figure 1 Schematic diagram of the Electromagnetic Borehole Flowmeter; reproduced from Molz and Young (1993). 


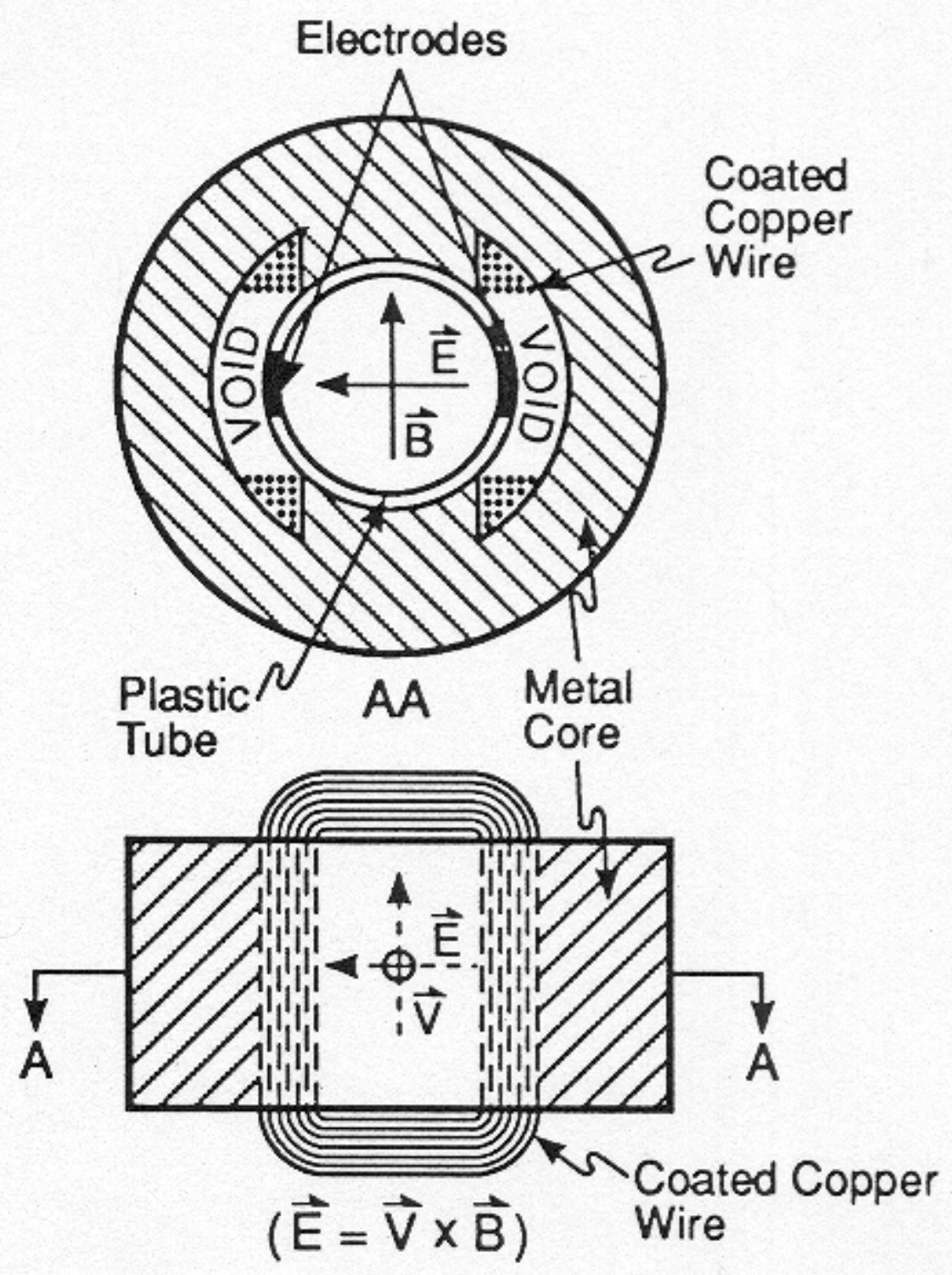

Figure 2 Electromagnetic Borehole Flowmeter (EBF) application of Faraday's Law of Induction; reproduced from Molz and Young (1993). 


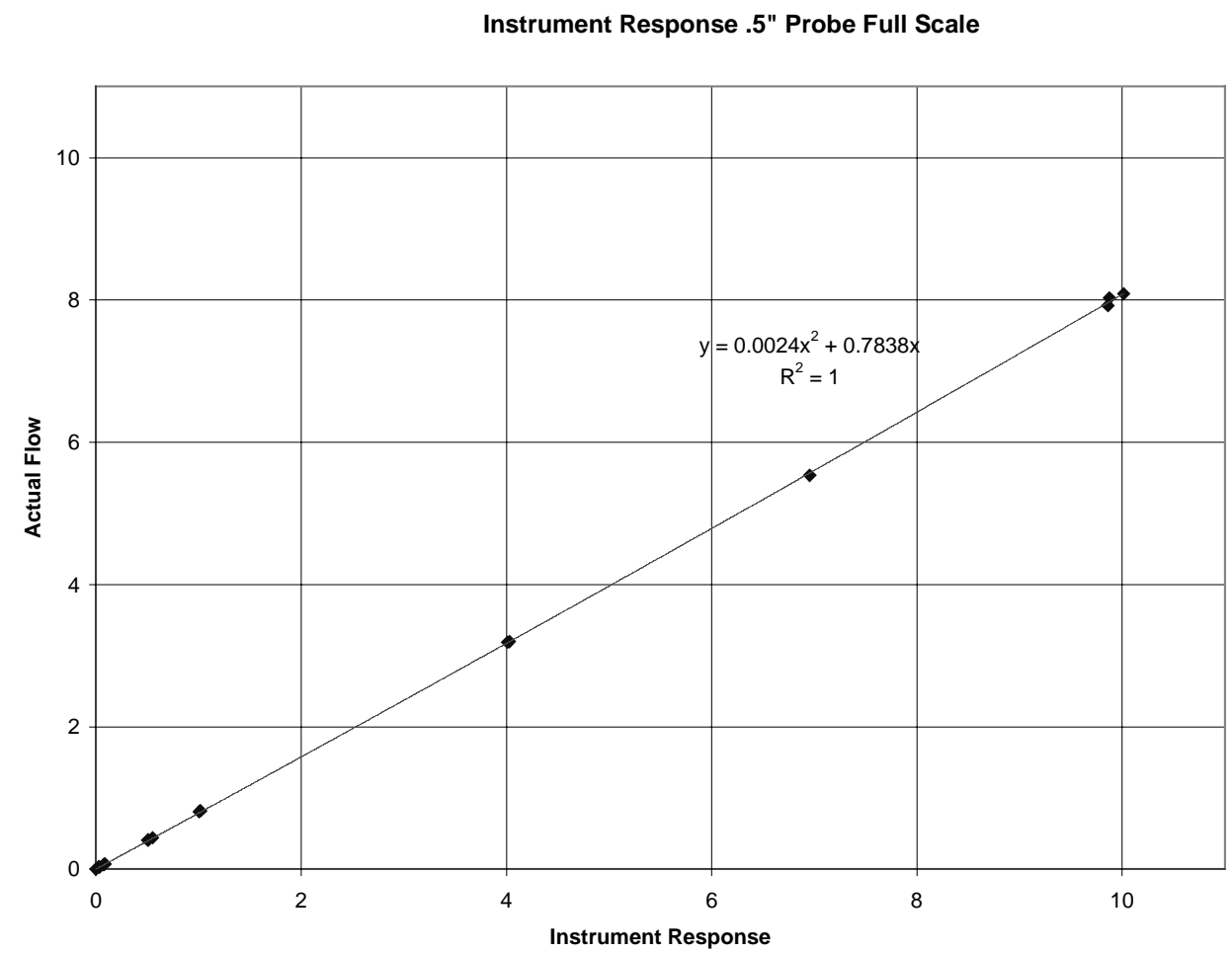

Figure 3 Calibration data and curve for the $1 / 2$ " ID EBF.

Instrument Response 1" Probe

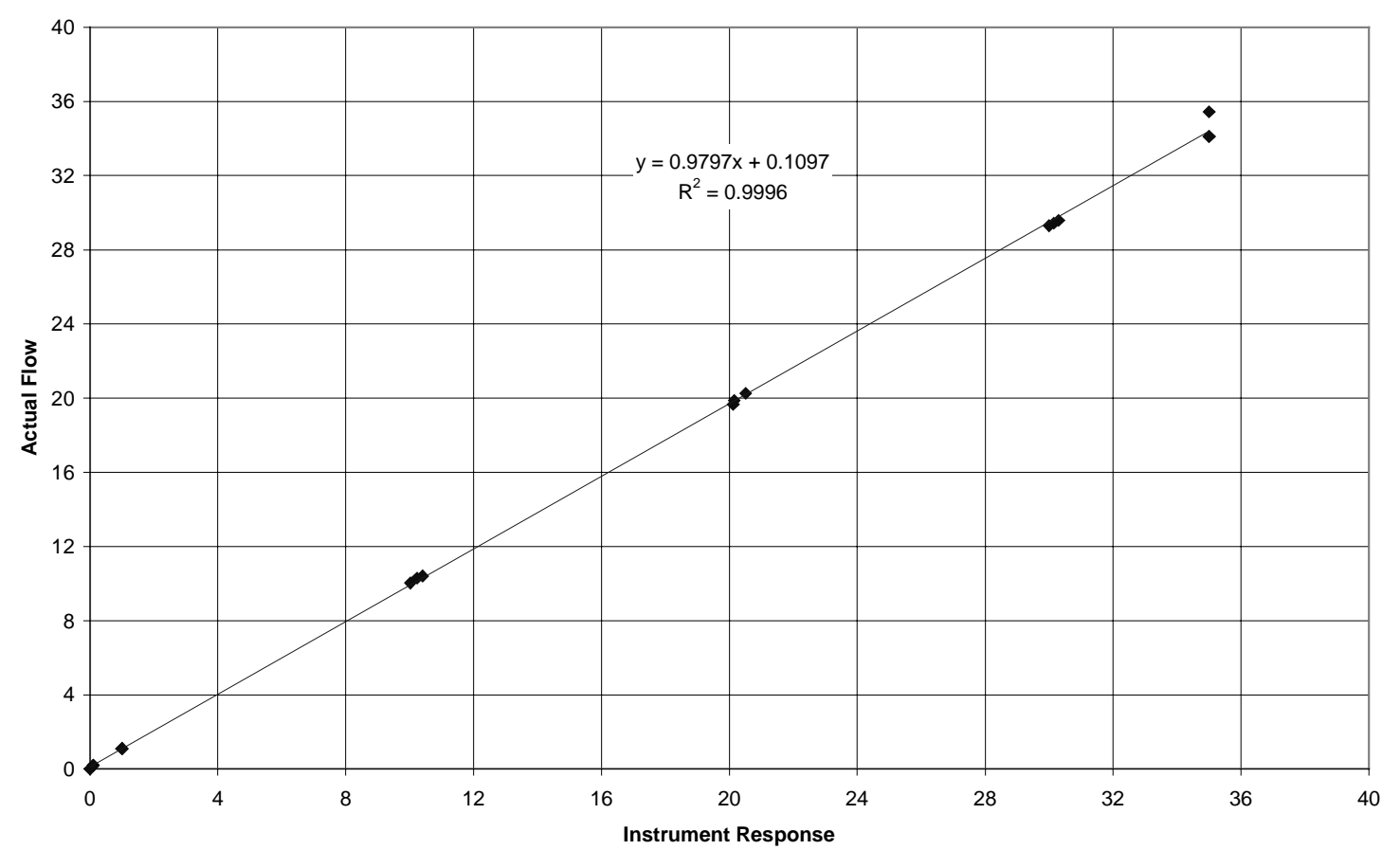

Figure 4 Calibration data and curve for the 1" ID EBF. 


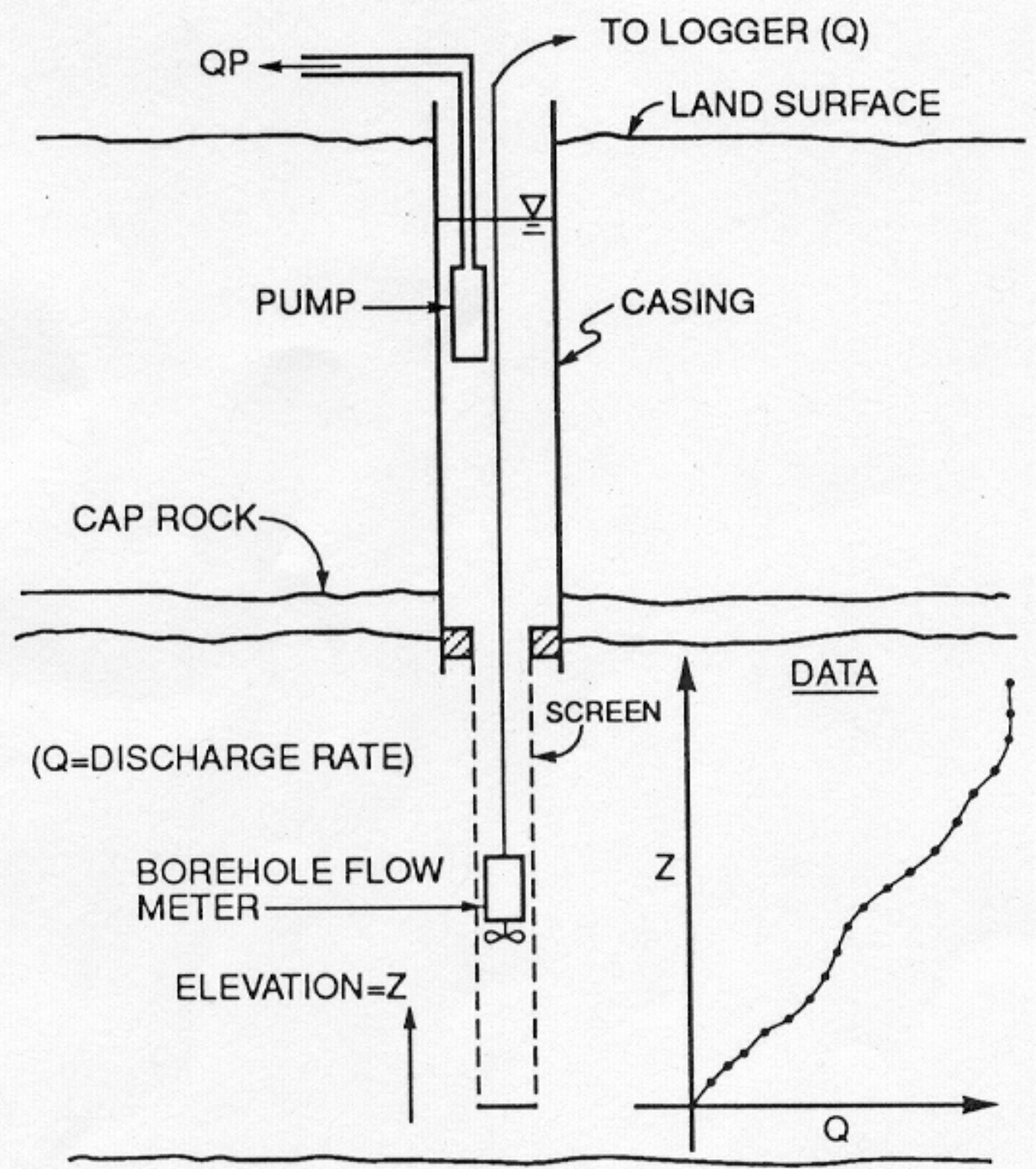

Figure 5 Schematic illustration of borehole flowmeter testing; reproduced from Molz and Young (1993). 


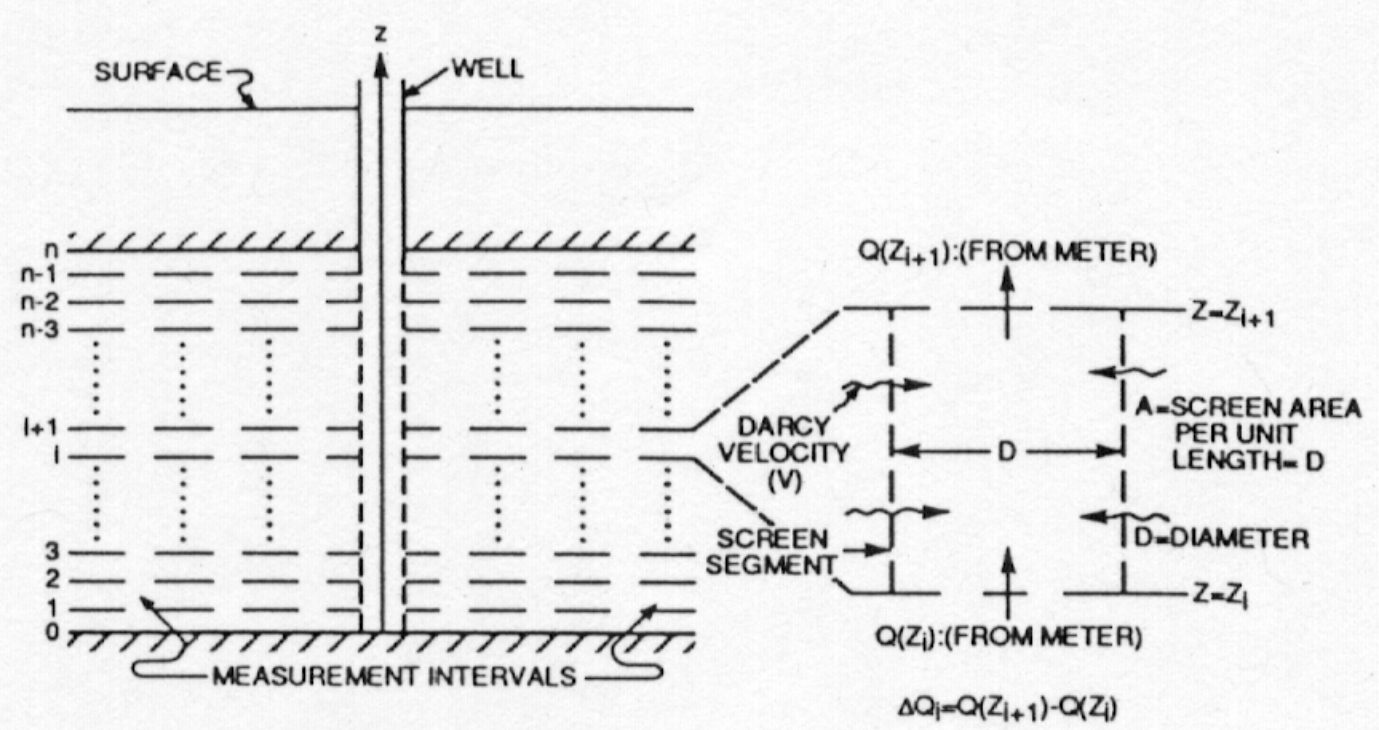

Figure 6 Basic geometry and analysis of borehole flowmeter data; reproduced from Molz and Young (1993). 


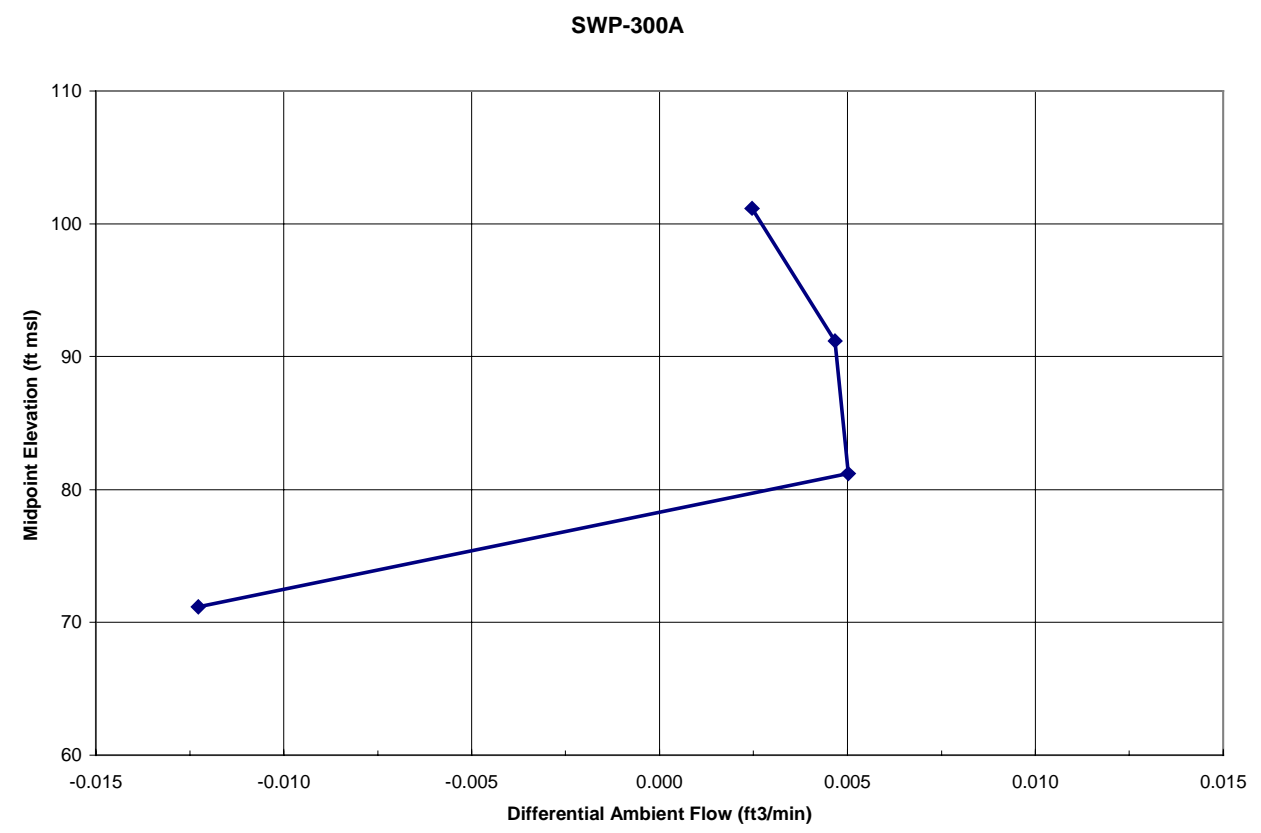

Figure 7 Ambient flow measurements for SWP-300A.

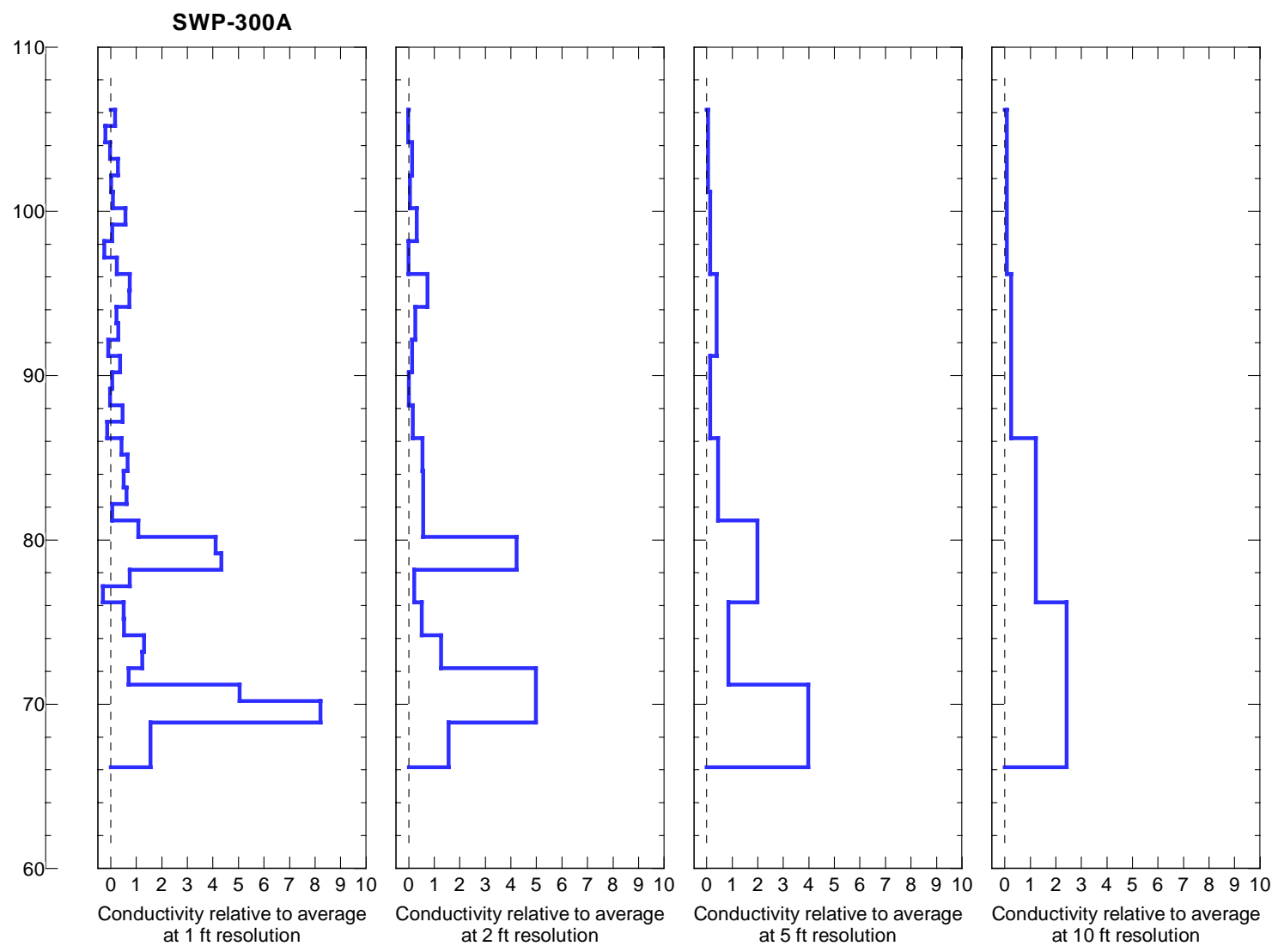

Figure 8 Estimated relative hydraulic conductivity profile for SWP-300A at 1, 2, 5 and $10 \mathrm{ft}$ resolutions. 


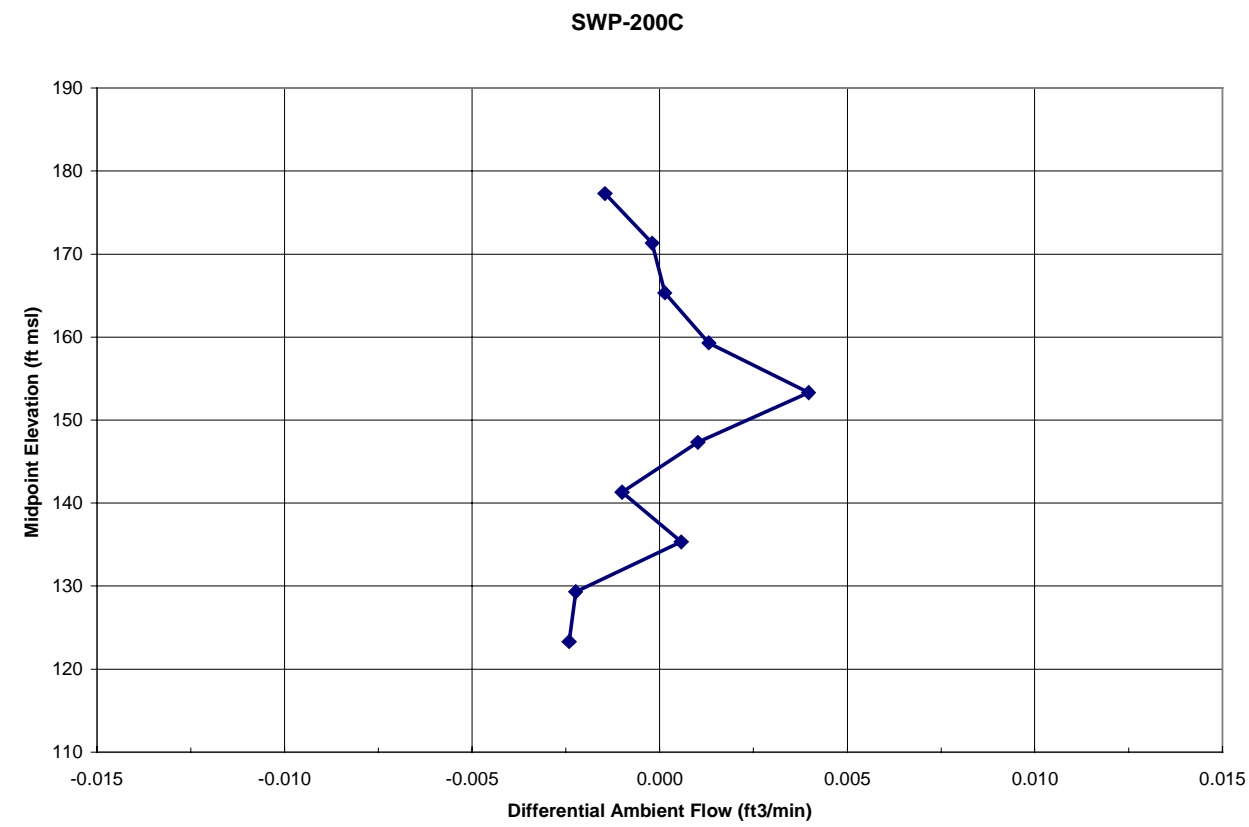

Figure 9 Ambient flow measurements for SWP-200C.

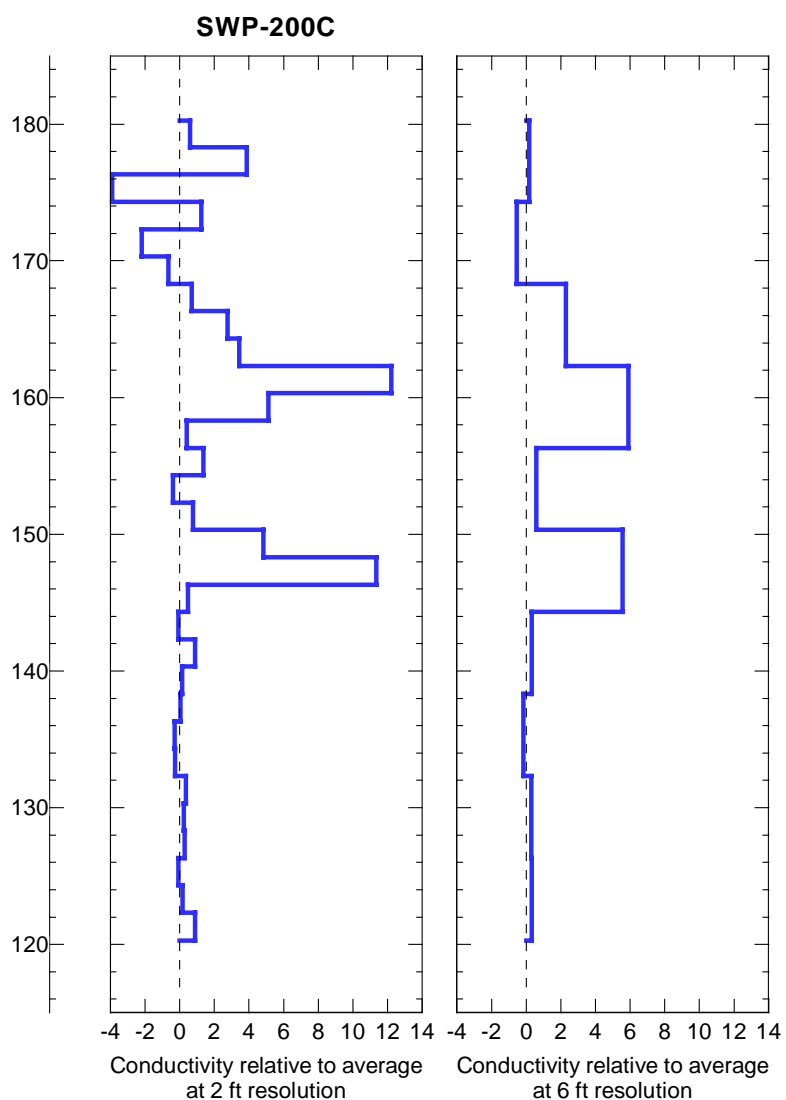

Figure 10 Estimated relative hydraulic conductivity profile for SWP-200C at 2 and 6 $\mathrm{ft}$ resolutions. 


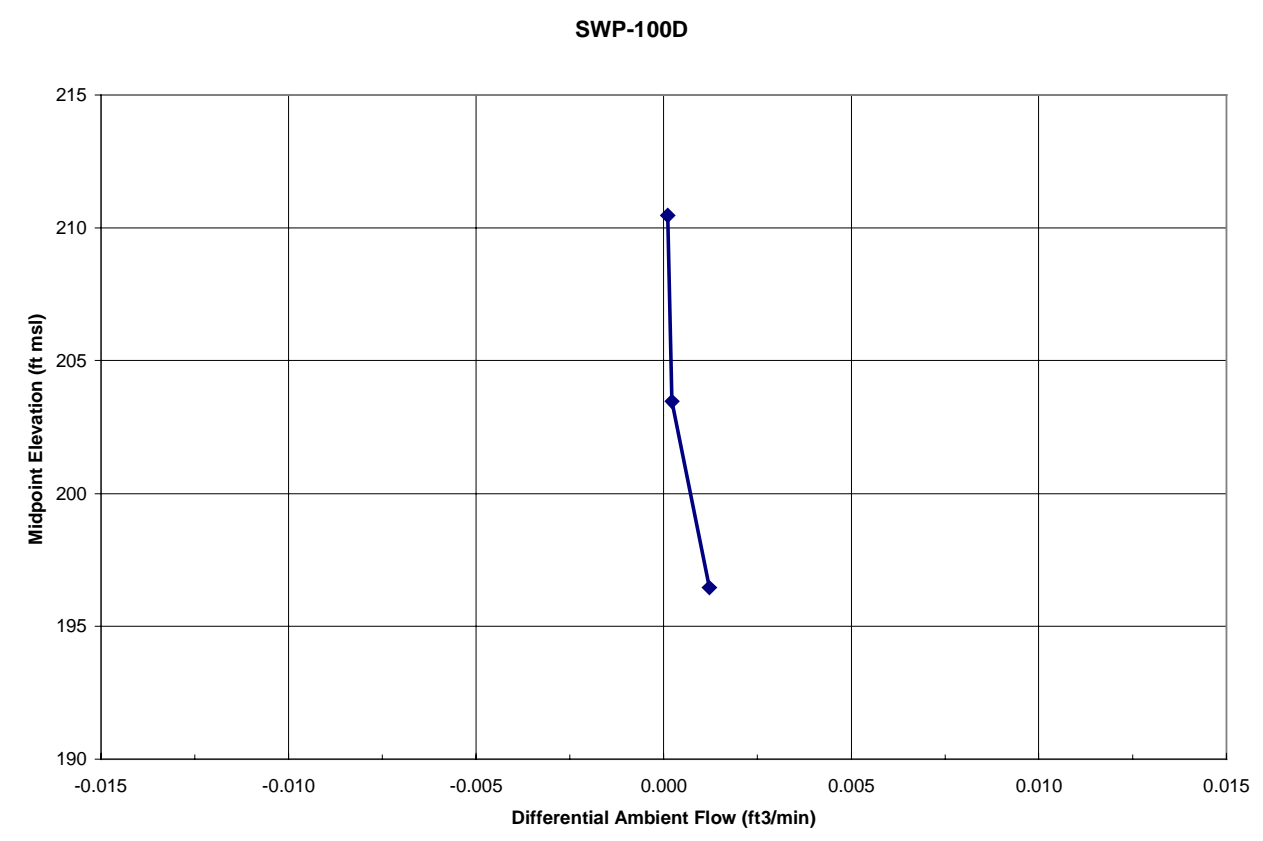

Figure 11 Ambient flow measurements for SWP-100D.
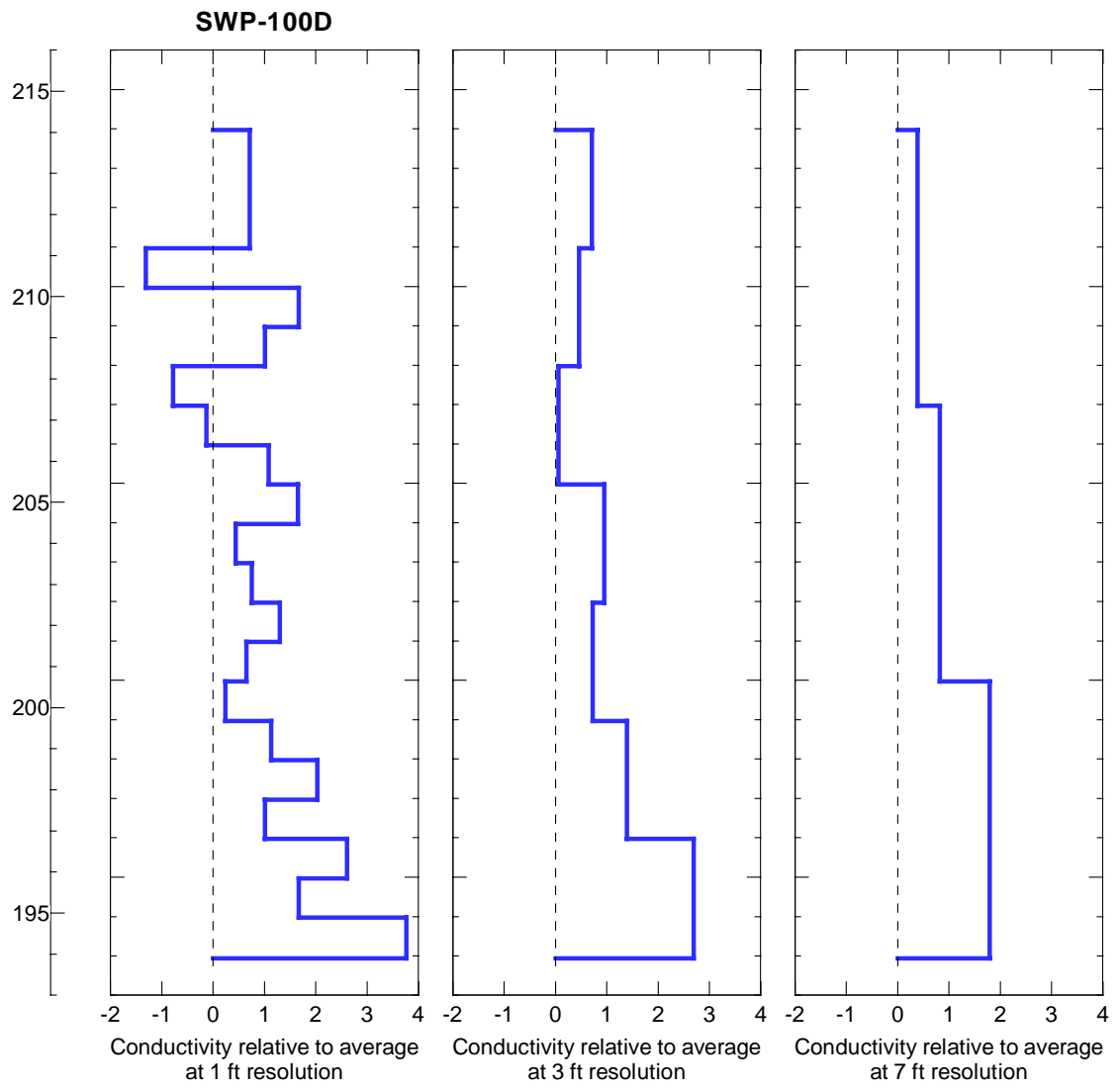

Figure 12 Estimated relative hydraulic conductivity profile for SWP-100D at 1, 3 and $7 \mathrm{ft}$ resolutions. 

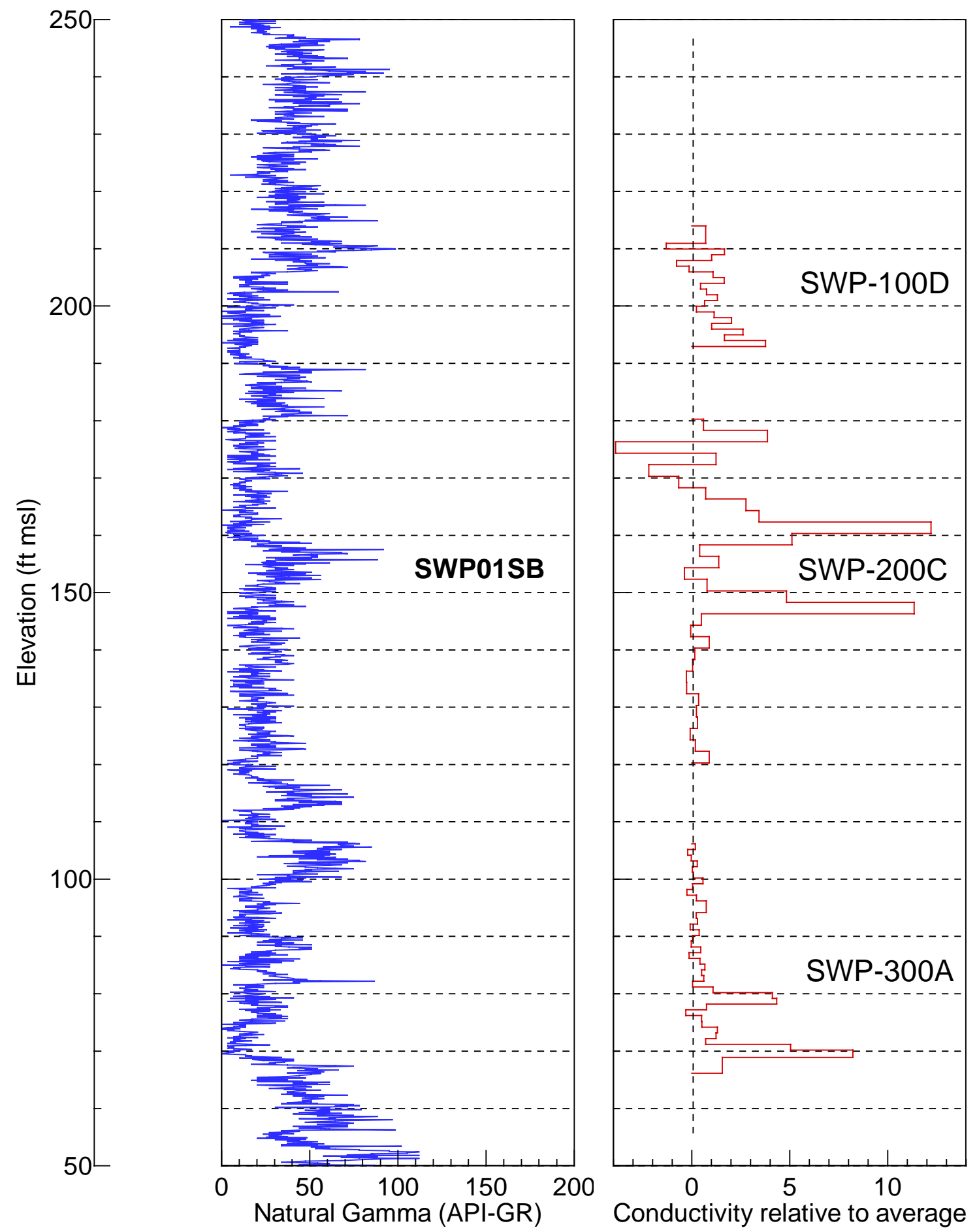

Figure 13 Comparison of EBF relative conductivity profiles to natural gamma log. 


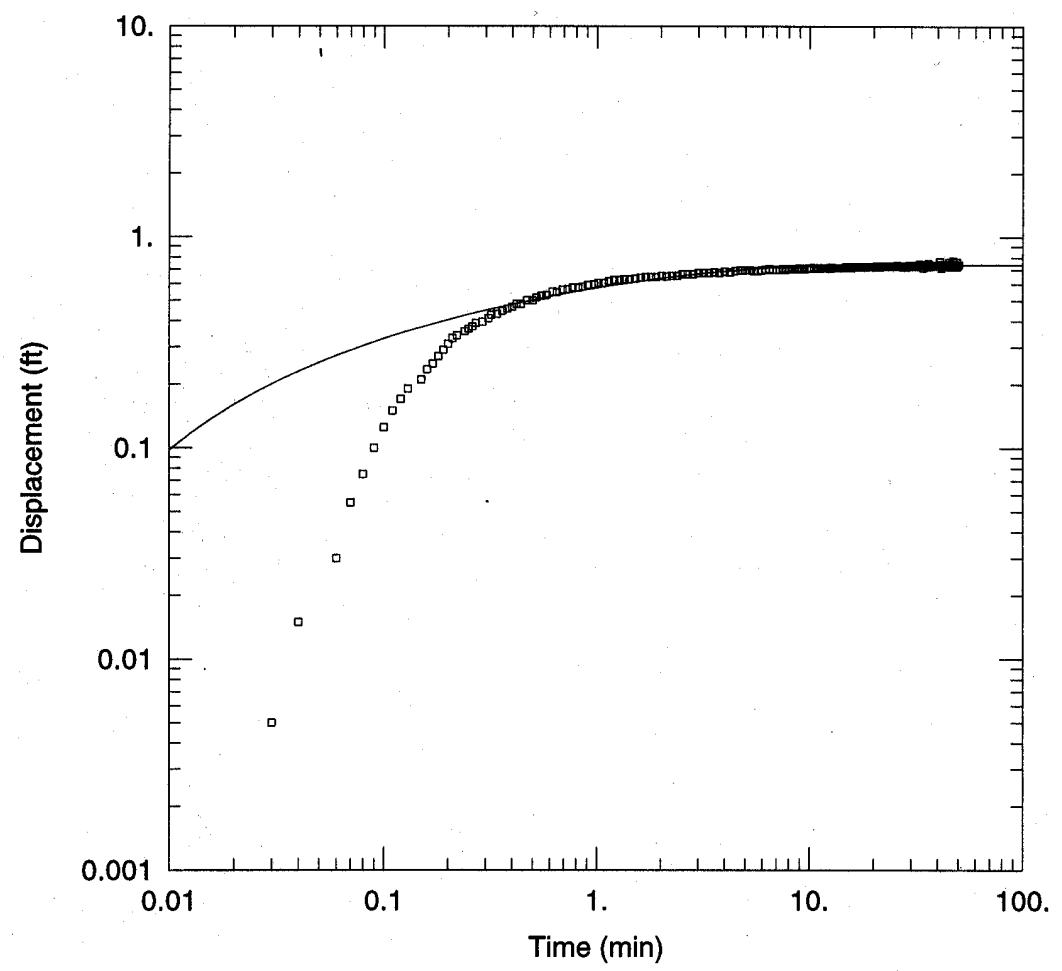

WELL TEST ANALYSIS

Data Set: C:11BobworkClBoreholeFlowlLogged DatalSWP300_compensated.aqt Date: $10 / 26 / 00 \quad$ Time: $14: 54: 30$

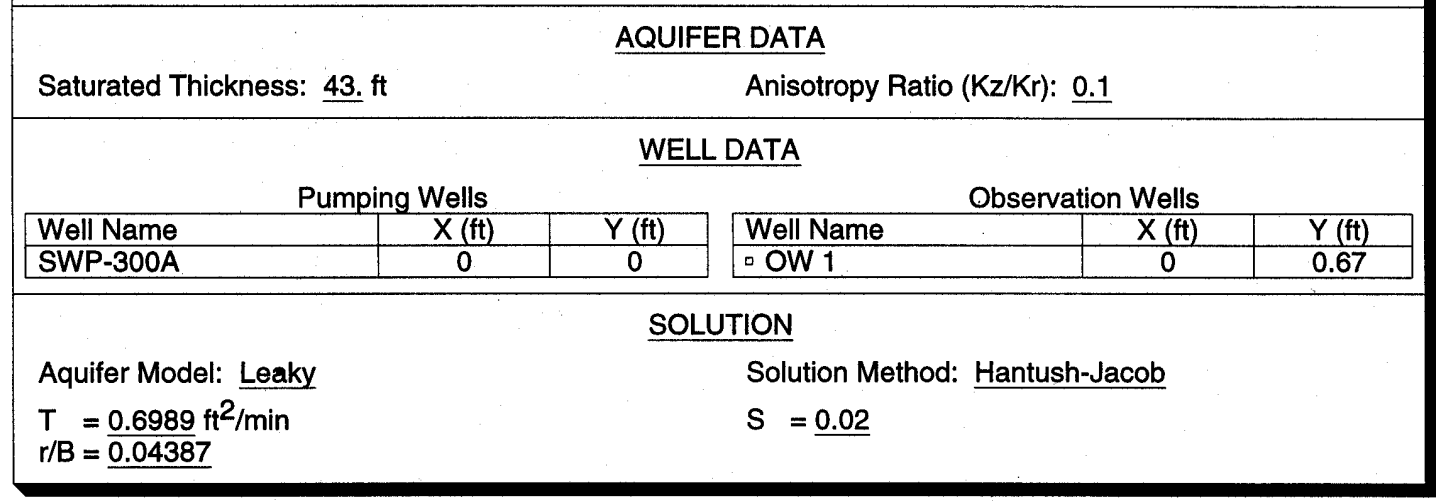

Figure 14 Time-drawdown data and single-well conductivity estimates for SWP-300A. 


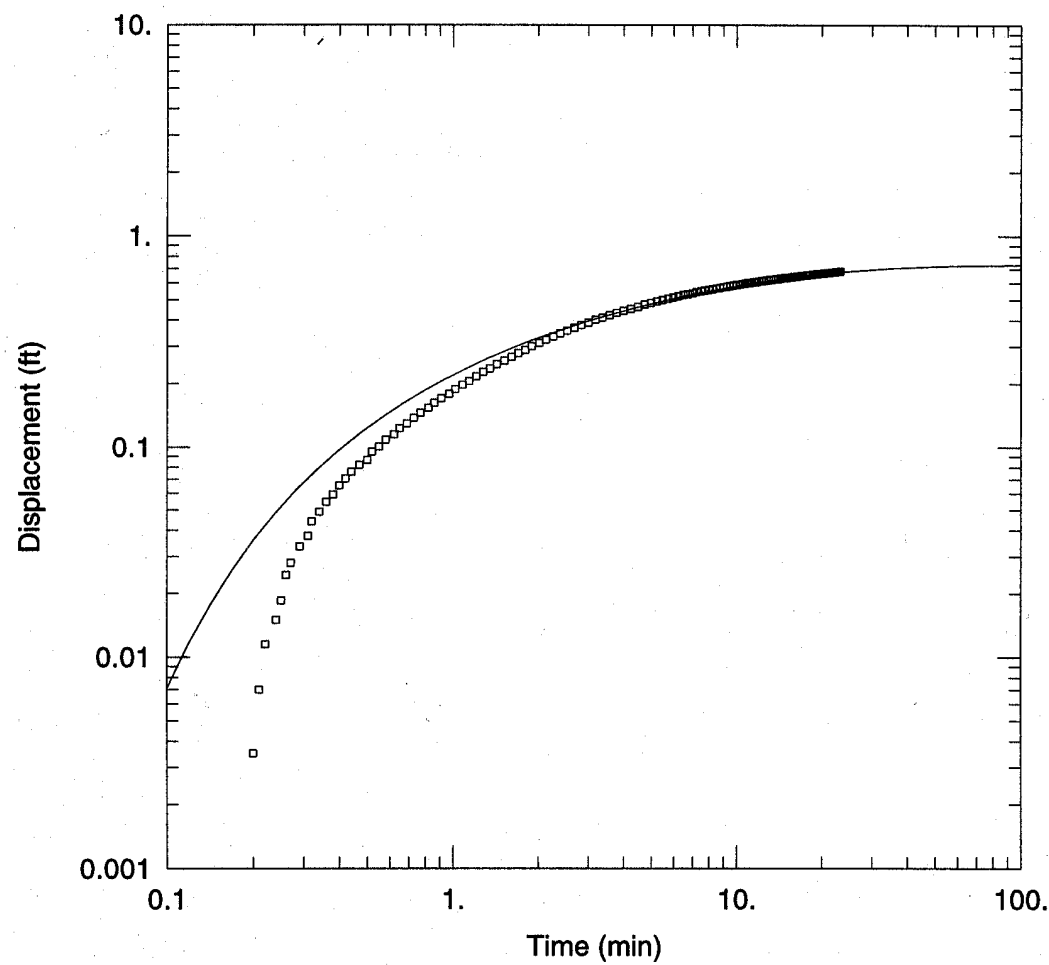

SWP-200C

Data Set: C:I1BobworkC\BoreholeFlowlLogged DatalSWP200C_compensated.aqt

Date: $10 / 26 / 00 \quad$ Time: $15: 12: 34$

AQUIFER DATA

Saturated Thickness: $65 . \mathrm{ft}$

Anisotropy Ratio $(\mathrm{Kz} / \mathrm{Kr}): \underline{0.1}$

WELL DATA

Pumping Wells
\begin{tabular}{|l|c|c|}
\hline Well Name & $\mathrm{X}(\mathrm{ft})$ & $\mathrm{Y}(\mathrm{ft})$ \\
\hline SWP-200C & 0 & 0 \\
\hline
\end{tabular}

Observation Wells
\begin{tabular}{|l|c|c|}
\hline Well Name & $\mathrm{X}(\mathrm{ft})$ & $\mathrm{Y}(\mathrm{ft})$ \\
\hline o OW 1 & 0 & 0.833 \\
\hline
\end{tabular}

SOLUTION

Aquifer Model: Leaky

Solution Method: Hantush-Jacob

$T=0.1018 \mathrm{ft}^{2} / \mathrm{min}$

$S=\underline{0.13}$

$r / B=\underline{0.1761}$

Figure 15 Time-drawdown data and single-well conductivity estimates for SWP-200C. 


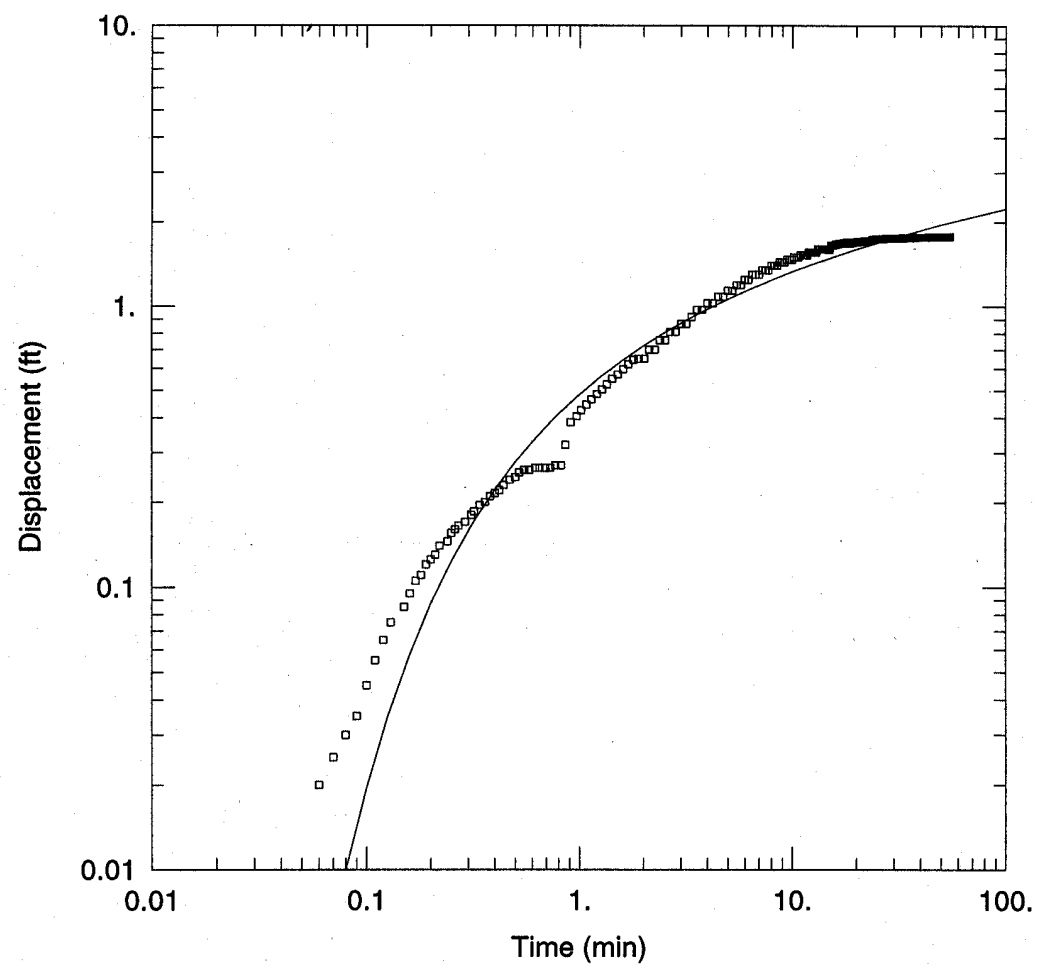

SWP-100D

Data Set: C:I1BobworkClBoreholeFlowlLogged DatalSWP100D_compensated.aqt Date: $10 / 26 / 00 \quad$ Time: $15: 29: 04$

AQUIFER DATA

Saturated Thickness: $\underline{24.3} \mathrm{ft}$

WELL DATA

Pumping Wells
\begin{tabular}{|l|c|c|}
\hline Well Name & $X(f t)$ & $Y(f t)$ \\
\hline SWP-100D & 0 & 0 \\
\hline
\end{tabular}

\begin{tabular}{|l|r|}
\multicolumn{2}{c}{ Observation Wells } \\
\hline Well Name & $\mathrm{X}(\mathrm{ft})$ \\
\hline o OW 1 & 0 \\
\hline
\end{tabular}

SOLUTION

Aquifer Model: Unconfined

Solution Method: Quick Neuman

$\mathrm{T}=\underline{0.2014} \mathrm{ft}^{2} / \mathrm{min}$

Sy $=\underline{0.001}$

$S=\underline{0.2311}$

$B=\underline{0.001}$

Figure 16 Time-drawdown data and single-well conductivity estimates for SWP-100D. 


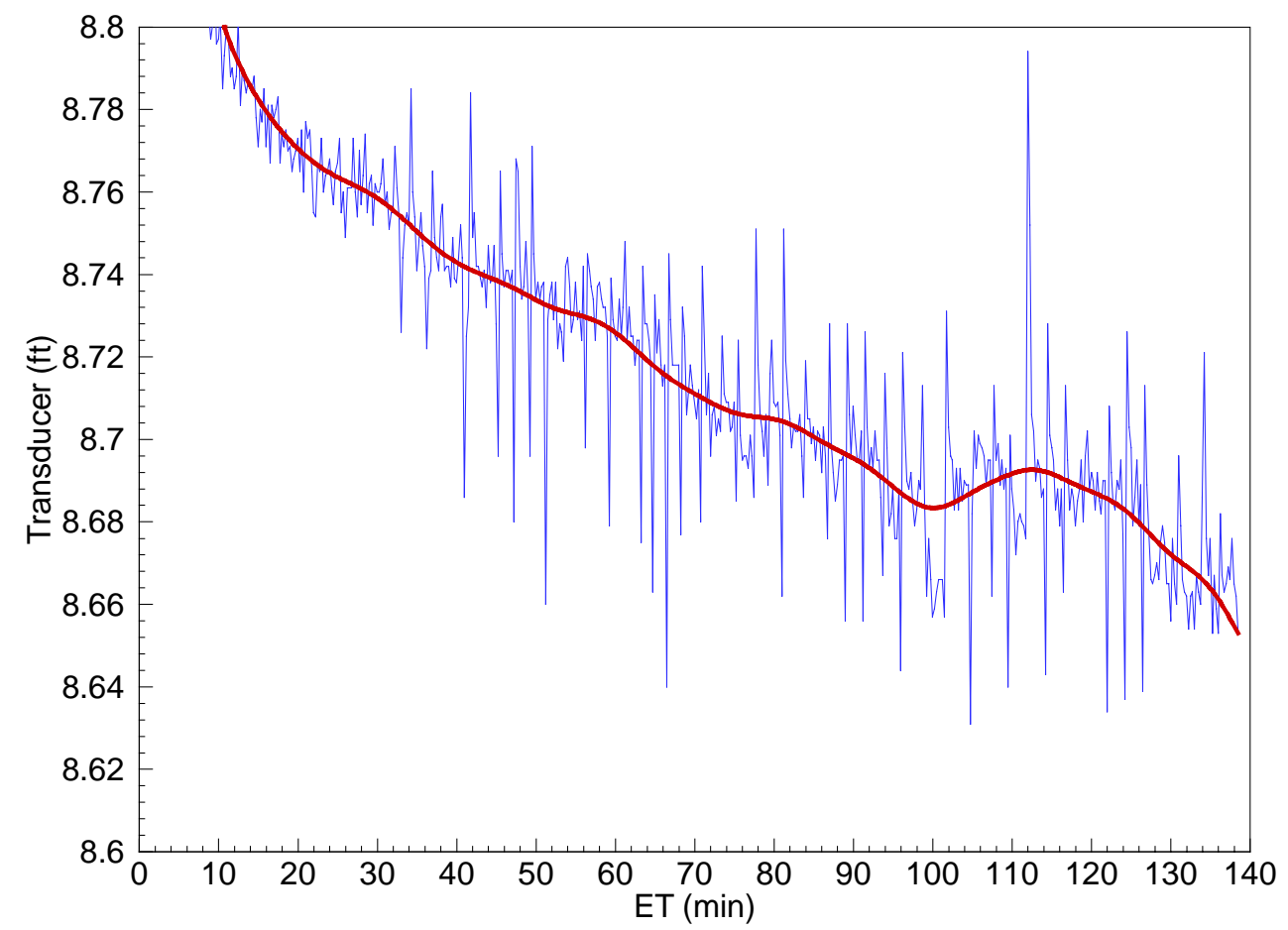

Figure 17 Unaltered and smoothed water level transducer data for SWP-300A.

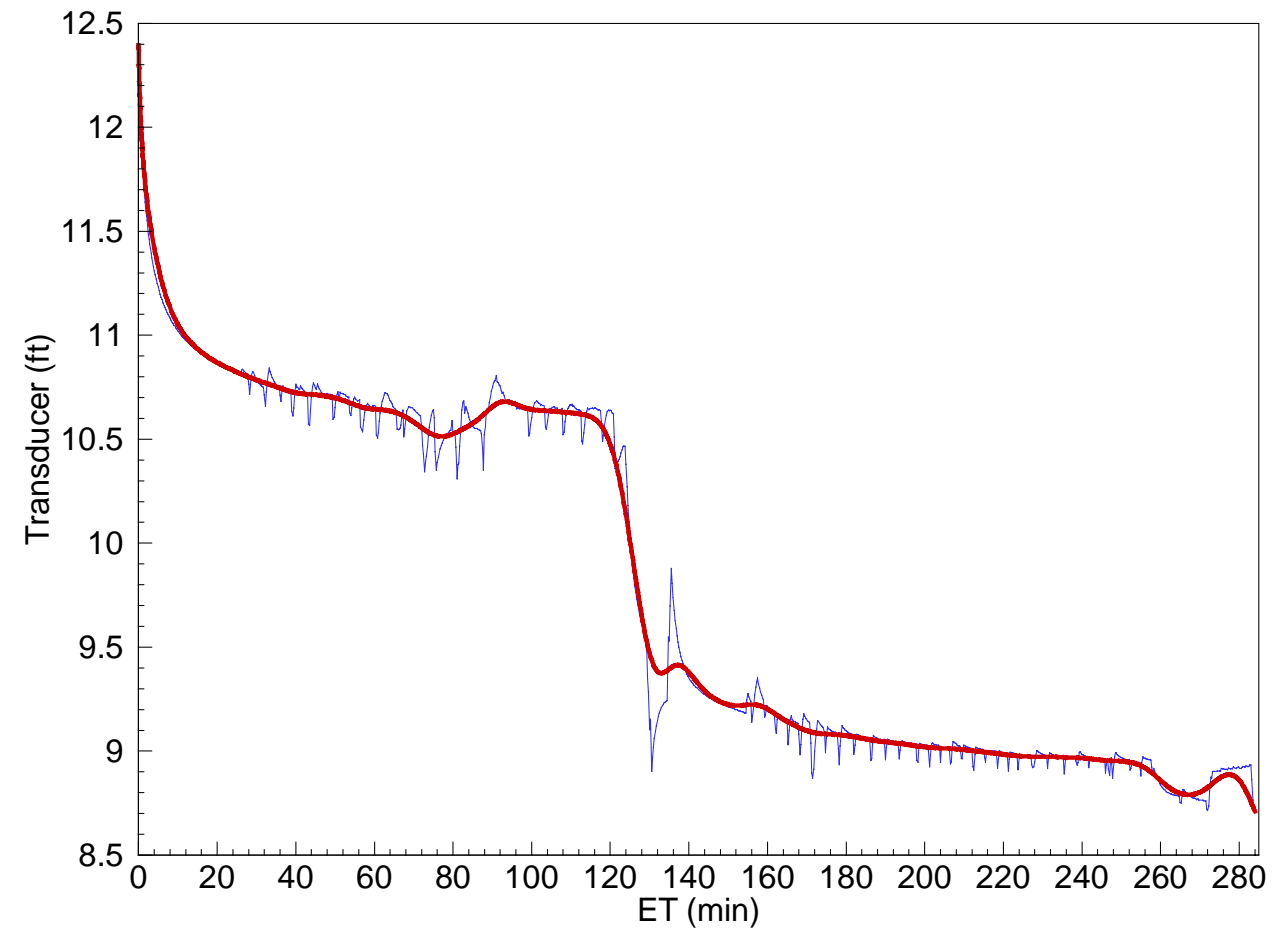

Figure 18 Unaltered and smoothed water level transducer data for SWP-200C. 


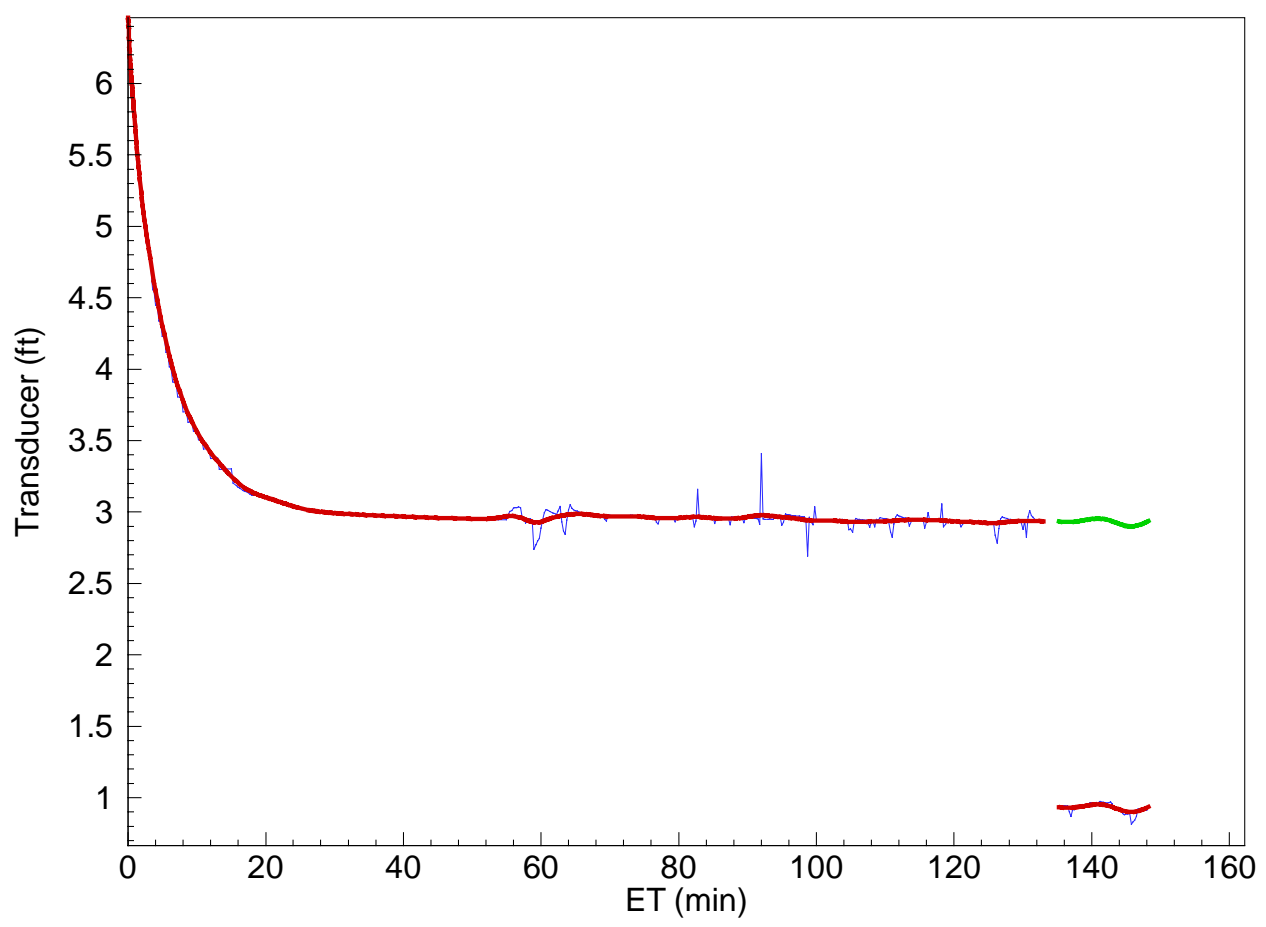

Figure 19 Unaltered and smoothed water level transducer data for SWP-100D. 


\section{Appendix A - Time-drawdown data for SWP-300A}

SPW 300 A Dynamic

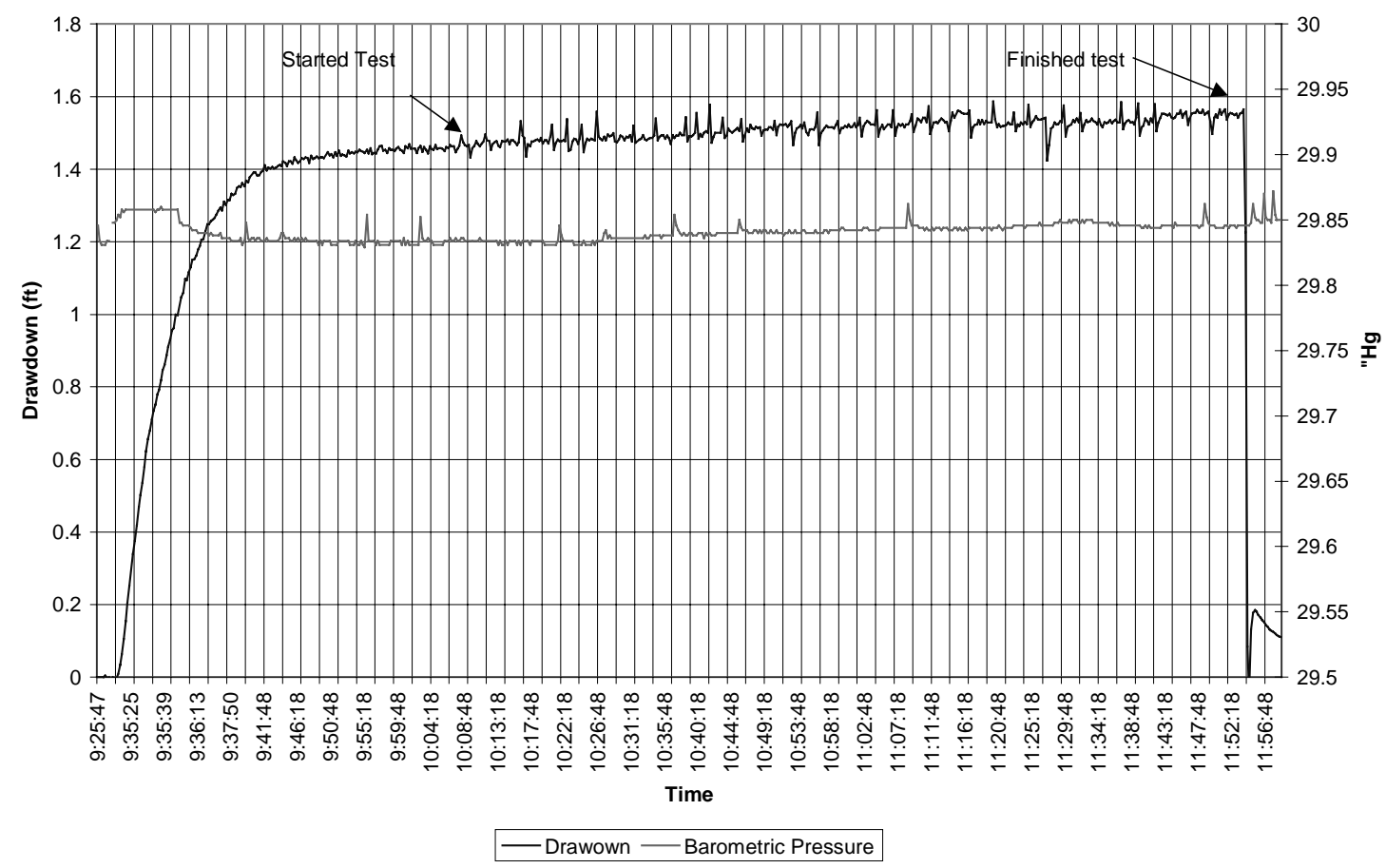

\begin{tabular}{|c|c|c|c|c|c|c|}
\hline & Date & Time & $\mathrm{ET}(\mathrm{min})$ & Feet $\mathrm{H} 2 \mathrm{O}$ & Inches $\mathrm{Hg}$ & Drawdown \\
\hline \multirow[t]{7}{*}{ Background } & $07 / 25 / 00$ & $9: 25: 47$ & 0 & 10.218 & 29.846 & 0 \\
\hline & $07 / 25 / 00$ & $9: 26: 47$ & 1 & 10.218 & 29.834 & 0 \\
\hline & $07 / 25 / 00$ & $9: 27: 47$ & 2 & 10.218 & 29.831 & 0 \\
\hline & $07 / 25 / 00$ & $9: 28: 47$ & 3 & 10.218 & 29.831 & 0 \\
\hline & $07 / 25 / 00$ & $9: 29: 47$ & 4 & 10.213 & 29.831 & 0.005 \\
\hline & $07 / 25 / 00$ & $9: 30: 47$ & 5 & 10.22 & 29.834 & -0.002 \\
\hline & $07 / 25 / 00$ & $9: 31: 47$ & 6 & 10.218 & 29.834 & 0 \\
\hline \multirow[t]{13}{*}{ Pumping } & $07 / 25 / 00$ & $9: 35: 19$ & 0 & 10.218 & 29.848 & 0 \\
\hline & $07 / 25 / 00$ & 9:35:19 & 0.0112 & 10.22 & 29.848 & -0.002 \\
\hline & $07 / 25 / 00$ & 9:35:20 & 0.0223 & 10.22 & 29.85 & -0.002 \\
\hline & $07 / 25 / 00$ & $9: 35: 21$ & 0.0335 & 10.208 & 29.854 & 0.01 \\
\hline & $07 / 25 / 00$ & $9: 35: 21$ & 0.0447 & 10.184 & 29.852 & 0.034 \\
\hline & $07 / 25 / 00$ & 9:35:22 & 0.0558 & 10.155 & 29.858 & 0.063 \\
\hline & $07 / 25 / 00$ & $9: 35: 23$ & 0.067 & 10.113 & 29.856 & 0.105 \\
\hline & $07 / 25 / 00$ & $9: 35: 23$ & 0.0782 & 10.064 & 29.858 & 0.154 \\
\hline & $07 / 25 / 00$ & 9:35:24 & 0.0893 & 10.014 & 29.858 & 0.204 \\
\hline & $07 / 25 / 00$ & 9:35:25 & 0.1005 & 9.966 & 29.858 & 0.252 \\
\hline & $07 / 25 / 00$ & $9: 35: 25$ & 0.1117 & 9.917 & 29.858 & 0.301 \\
\hline & $07 / 25 / 00$ & $9: 35: 26$ & 0.1228 & 9.878 & 29.858 & 0.34 \\
\hline & $07 / 25 / 00$ & 9:35:27 & 0.134 & 9.841 & 29.858 & 0.377 \\
\hline
\end{tabular}




\section{Appendix A (continued)}

\begin{tabular}{|c|c|c|c|c|c|}
\hline 07/25/00 & $9: 35: 27$ & 0.1452 & 9.798 & 29.858 & 0.42 \\
\hline 07/25/00 & $9: 35: 28$ & 0.1563 & 9.749 & 29.858 & 0.469 \\
\hline 07/25/00 & 9:35:29 & 0.1675 & 9.716 & 29.858 & 0.502 \\
\hline 07/25/00 & $9: 35: 29$ & 0.1787 & 9.681 & 29.858 & 0.537 \\
\hline 07/25/00 & 9:35:30 & 0.1898 & 9.638 & 29.858 & 0.58 \\
\hline 07/25/00 & 9:35:31 & 0.201 & 9.595 & 29.858 & 0.623 \\
\hline 07/25/00 & $9: 35: 31$ & 0.2122 & 9.562 & 29.858 & 0.656 \\
\hline 07/25/00 & 9:35:32 & 0.2233 & 9.537 & 29.858 & 0.681 \\
\hline 07/25/00 & $9: 35: 33$ & 0.235 & 9.507 & 29.858 & 0.711 \\
\hline $07 / 25 / 00$ & 9:35:33 & 0.2475 & 9.484 & 29.858 & 0.734 \\
\hline 07/25/00 & $9: 35: 34$ & 0.2607 & 9.467 & 29.856 & 0.751 \\
\hline 07/25/00 & 9:35:35 & 0.2747 & 9.438 & 29.858 & 0.78 \\
\hline 07/25/00 & $9: 35: 36$ & 0.2895 & 9.426 & 29.858 & 0.792 \\
\hline 07/25/00 & 9:35:37 & 0.3052 & 9.4 & 29.86 & 0.818 \\
\hline 07/25/00 & 9:35:38 & 0.3218 & 9.372 & 29.858 & 0.846 \\
\hline 07/25/00 & 9:35:39 & 0.3395 & 9.357 & 29.858 & 0.861 \\
\hline 07/25/00 & $9: 35: 40$ & 0.3582 & 9.331 & 29.858 & 0.887 \\
\hline 07/25/00 & 9:35:41 & 0.378 & 9.307 & 29.858 & 0.911 \\
\hline 07/25/00 & 9:35:42 & 0.399 & 9.287 & 29.858 & 0.931 \\
\hline 07/25/00 & $9: 35: 44$ & 0.4212 & 9.262 & 29.858 & 0.956 \\
\hline 07/25/00 & $9: 35: 45$ & 0.4447 & 9.256 & 29.858 & 0.962 \\
\hline $07 / 25 / 00$ & $9: 35: 47$ & 0.4695 & 9.219 & 29.858 & 0.999 \\
\hline 07/25/00 & 9:35:48 & 0.4958 & 9.22 & 29.858 & 0.998 \\
\hline 07/25/00 & 9:35:50 & 0.5238 & 9.192 & 29.848 & 1.026 \\
\hline 07/25/00 & 9:35:52 & 0.5535 & 9.171 & 29.848 & 1.047 \\
\hline $07 / 25 / 00$ & $9: 35: 54$ & 0.5848 & 9.158 & 29.846 & 1.06 \\
\hline 07/25/00 & $9: 35: 56$ & 0.618 & 9.121 & 29.846 & 1.097 \\
\hline 07/25/00 & 9:35:58 & 0.6532 & 9.124 & 29.846 & 1.094 \\
\hline 07/25/00 & 9:36:00 & 0.6905 & 9.102 & 29.846 & 1.116 \\
\hline 07/25/00 & 9:36:02 & 0.73 & 9.088 & 29.844 & 1.13 \\
\hline 07/25/00 & 9:36:05 & 0.7718 & 9.068 & 29.842 & 1.15 \\
\hline 07/25/00 & 9:36:07 & 0.8162 & 9.066 & 29.842 & 1.152 \\
\hline 07/25/00 & $9: 36: 10$ & 0.8632 & 9.056 & 29.842 & 1.162 \\
\hline $07 / 25 / 00$ & $9: 36: 13$ & 0.913 & 9.039 & 29.84 & 1.179 \\
\hline 07/25/00 & $9: 36: 16$ & 0.9657 & 9.03 & 29.84 & 1.188 \\
\hline 07/25/00 & 9:36:20 & 1.0215 & 9.013 & 29.84 & 1.205 \\
\hline 07/25/00 & $9: 36: 23$ & 1.0807 & 9.01 & 29.84 & 1.208 \\
\hline 07/25/00 & $9: 36: 27$ & 1.1433 & 8.989 & 29.84 & 1.229 \\
\hline 07/25/00 & $9: 36: 31$ & 1.2097 & 8.97 & 29.84 & 1.248 \\
\hline 07/25/00 & $9: 36: 35$ & 1.28 & 8.97 & 29.838 & 1.248 \\
\hline 07/25/00 & 9:36:40 & 1.3545 & 8.961 & 29.84 & 1.257 \\
\hline 07/25/00 & 9:36:45 & 1.4335 & 8.957 & 29.838 & 1.261 \\
\hline 07/25/00 & $9: 36: 50$ & 1.5172 & 8.953 & 29.838 & 1.265 \\
\hline 07/25/00 & $9: 36: 55$ & 1.6057 & 8.942 & 29.838 & 1.276 \\
\hline 07/25/00 & $9: 37: 00$ & 1.6995 & 8.931 & 29.838 & 1.287 \\
\hline $07 / 25 / 00$ & 9:37:06 & 1.7988 & 8.924 & 29.84 & 1.294 \\
\hline 07/25/00 & $9: 37: 13$ & 1.9042 & 8.931 & 29.836 & 1.287 \\
\hline 07/25/00 & 9:37:19 & 2.0157 & 8.908 & 29.836 & 1.31 \\
\hline 07/25/00 & $9: 37: 27$ & 2.1338 & 8.917 & 29.836 & 1.301 \\
\hline 07/25/00 & $9: 37: 34$ & 2.259 & 8.904 & 29.836 & 1.314 \\
\hline 07/25/00 & $9: 37: 42$ & 2.3915 & 8.904 & 29.836 & 1.31 \\
\hline
\end{tabular}


Appendix A (continued)

\begin{tabular}{|c|c|c|c|c|c|}
\hline $07 / 25 / 00$ & $9: 37: 50$ & 2.532 & 8.886 & 29.834 & 1.332 \\
\hline $07 / 25 / 00$ & $9: 37: 59$ & 2.6808 & 8.889 & 29.834 & 1.329 \\
\hline $07 / 25 / 00$ & 9:38:09 & 2.8383 & 8.886 & 29.834 & 1.332 \\
\hline $07 / 25 / 00$ & $9: 38: 19$ & 3.0052 & 8.87 & 29.834 & 1.348 \\
\hline $07 / 25 / 00$ & 9:38:29 & 3.182 & 8.865 & 29.834 & 1.353 \\
\hline 07/25/00 & $9: 38: 41$ & 3.3693 & 8.868 & 29.836 & 1.35 \\
\hline $07 / 25 / 00$ & $9: 38: 53$ & 3.5677 & 8.856 & 29.831 & 1.362 \\
\hline $07 / 25 / 00$ & 9:39:05 & 3.7778 & 8.865 & 29.834 & 1.353 \\
\hline 07/25/00 & $9: 39: 19$ & 4.0005 & 8.85 & 29.848 & 1.368 \\
\hline $07 / 25 / 00$ & 9:39:33 & 4.2363 & 8.855 & 29.836 & 1.363 \\
\hline 07/25/00 & $9: 39: 48$ & 4.4862 & 8.84 & 29.834 & 1.378 \\
\hline $07 / 25 / 00$ & $9: 40: 03$ & 4.7362 & 8.833 & 29.836 & 1.385 \\
\hline $07 / 25 / 00$ & $9: 40: 18$ & 4.9862 & 8.827 & 29.836 & 1.391 \\
\hline 07/25/00 & $9: 40: 33$ & 5.2362 & 8.827 & 29.834 & 1.391 \\
\hline $07 / 25 / 00$ & $9: 40: 48$ & 5.4862 & 8.836 & 29.836 & 1.382 \\
\hline $07 / 25 / 00$ & $9: 41: 03$ & 5.7362 & 8.834 & 29.836 & 1.384 \\
\hline $07 / 25 / 00$ & $9: 41: 18$ & 5.9862 & 8.826 & 29.834 & 1.392 \\
\hline 07/25/00 & $9: 41: 33$ & 6.2362 & 8.823 & 29.834 & 1.395 \\
\hline 07/25/00 & $9: 41: 48$ & 6.4862 & 8.807 & 29.834 & 1.411 \\
\hline $07 / 25 / 00$ & $9: 42: 03$ & 6.7362 & 8.821 & 29.836 & 1.397 \\
\hline $07 / 25 / 00$ & $9: 42: 18$ & 6.9862 & 8.814 & 29.834 & 1.404 \\
\hline 07/25/00 & $9: 42: 33$ & 7.2362 & 8.816 & 29.834 & 1.402 \\
\hline $07 / 25 / 00$ & $9: 42: 48$ & 7.4862 & 8.811 & 29.834 & 1.407 \\
\hline $07 / 25 / 00$ & $9: 43: 03$ & 7.7362 & 8.816 & 29.834 & 1.402 \\
\hline 07/25/00 & $9: 43: 18$ & 7.9862 & 8.813 & 29.834 & 1.405 \\
\hline $07 / 25 / 00$ & $9: 43: 33$ & 8.2362 & 8.809 & 29.834 & 1.409 \\
\hline $07 / 25 / 00$ & $9: 43: 48$ & 8.4862 & 8.806 & 29.836 & 1.412 \\
\hline $07 / 25 / 00$ & $9: 44: 03$ & 8.7362 & 8.81 & 29.84 & 1.408 \\
\hline $07 / 25 / 00$ & $9: 44: 18$ & 8.9862 & 8.797 & 29.84 & 1.421 \\
\hline $07 / 25 / 00$ & $9: 44: 33$ & 9.2362 & 8.8 & 29.836 & 1.418 \\
\hline $07 / 25 / 00$ & $9: 44: 48$ & 9.4862 & 8.81 & 29.836 & 1.408 \\
\hline 07/25/00 & $9: 45: 03$ & 9.7362 & 8.796 & 29.836 & 1.422 \\
\hline 07/25/00 & $9: 45: 18$ & 9.9862 & 8.797 & 29.834 & 1.421 \\
\hline $07 / 25 / 00$ & $9: 45: 33$ & 10.2362 & 8.803 & 29.834 & 1.415 \\
\hline $07 / 25 / 00$ & $9: 45: 48$ & 10.4862 & 8.785 & 29.836 & 1.433 \\
\hline 07/25/00 & $9: 46: 03$ & 10.7362 & 8.793 & 29.834 & 1.425 \\
\hline $07 / 25 / 00$ & $9: 46: 18$ & 10.9862 & 8.8 & 29.836 & 1.418 \\
\hline 07/25/00 & $9: 46: 33$ & 11.2362 & 8.797 & 29.834 & 1.421 \\
\hline 07/25/00 & $9: 46: 48$ & 11.4862 & 8.788 & 29.836 & 1.43 \\
\hline $07 / 25 / 00$ & $9: 47: 03$ & 11.7362 & 8.79 & 29.834 & 1.428 \\
\hline $07 / 25 / 00$ & $9: 47: 18$ & 11.9862 & 8.785 & 29.836 & 1.433 \\
\hline 07/25/00 & $9: 47: 33$ & 12.2362 & 8.788 & 29.834 & 1.43 \\
\hline $07 / 25 / 00$ & $9: 47: 48$ & 12.4862 & 8.801 & 29.834 & 1.417 \\
\hline $07 / 25 / 00$ & $9: 48: 03$ & 12.7362 & 8.781 & 29.834 & 1.437 \\
\hline $07 / 25 / 00$ & $9: 48: 18$ & 12.9862 & 8.79 & 29.834 & 1.428 \\
\hline $07 / 25 / 00$ & $9: 48: 33$ & 13.2362 & 8.787 & 29.834 & 1.431 \\
\hline 07/25/00 & $9: 48: 48$ & 13.4862 & 8.784 & 29.834 & 1.434 \\
\hline $07 / 25 / 00$ & $9: 49: 03$ & 13.7362 & 8.787 & 29.831 & 1.431 \\
\hline $07 / 25 / 00$ & $9: 49: 18$ & 13.9862 & 8.785 & 29.834 & 1.433 \\
\hline $07 / 25 / 00$ & $9: 49: 33$ & 14.2362 & 8.785 & 29.831 & 1.433 \\
\hline 07/25/00 & $9: 49: 48$ & 14.4862 & 8.788 & 29.834 & 1.43 \\
\hline
\end{tabular}




\section{Appendix A (continued)}

\begin{tabular}{|c|c|c|c|c|c|}
\hline $07 / 25 / 00$ & 9:50:03 & 14.7362 & 8.778 & 29.834 & 1.44 \\
\hline $07 / 25 / 00$ & $9: 50: 18$ & 14.9862 & 8.771 & 29.834 & 1.447 \\
\hline $07 / 25 / 00$ & $9: 50: 33$ & 15.2362 & 8.78 & 29.834 & 1.438 \\
\hline $07 / 25 / 00$ & $9: 50: 48$ & 15.4862 & 8.777 & 29.831 & 1.441 \\
\hline $07 / 25 / 00$ & $9: 51: 03$ & 15.7362 & 8.785 & 29.831 & 1.433 \\
\hline $07 / 25 / 00$ & $9: 51: 18$ & 15.9862 & 8.771 & 29.831 & 1.447 \\
\hline $07 / 25 / 00$ & $9: 51: 33$ & 16.2362 & 8.781 & 29.831 & 1.437 \\
\hline $07 / 25 / 00$ & $9: 51: 48$ & 16.4862 & 8.767 & 29.834 & 1.451 \\
\hline $07 / 25 / 00$ & $9: 52: 03$ & 16.7362 & 8.781 & 29.834 & 1.437 \\
\hline $07 / 25 / 00$ & $9: 52: 18$ & 16.9862 & 8.778 & 29.834 & 1.44 \\
\hline $07 / 25 / 00$ & 9:52:33 & 17.2362 & 8.78 & 29.834 & 1.438 \\
\hline $07 / 25 / 00$ & $9: 52: 48$ & 17.4862 & 8.783 & 29.834 & 1.435 \\
\hline $07 / 25 / 00$ & 9:53:03 & 17.7362 & 8.767 & 29.834 & 1.451 \\
\hline $07 / 25 / 00$ & 9:53:18 & 17.9862 & 8.774 & 29.831 & 1.444 \\
\hline $07 / 25 / 00$ & $9: 53: 33$ & 18.2362 & 8.771 & 29.831 & 1.447 \\
\hline $07 / 25 / 00$ & $9: 53: 48$ & 18.4862 & 8.775 & 29.831 & 1.443 \\
\hline $07 / 25 / 00$ & $9: 54: 03$ & 18.7362 & 8.77 & 29.834 & 1.448 \\
\hline $07 / 25 / 00$ & $9: 54: 18$ & 18.9862 & 8.771 & 29.831 & 1.447 \\
\hline $07 / 25 / 00$ & $9: 54: 33$ & 19.2362 & 8.765 & 29.831 & 1.453 \\
\hline $07 / 25 / 00$ & $9: 54: 48$ & 19.4862 & 8.768 & 29.834 & 1.45 \\
\hline $07 / 25 / 00$ & $9: 55: 03$ & 19.7362 & 8.77 & 29.831 & 1.448 \\
\hline $07 / 25 / 00$ & $9: 55: 18$ & 19.9862 & 8.773 & 29.829 & 1.445 \\
\hline $07 / 25 / 00$ & $9: 55: 33$ & 20.2362 & 8.765 & 29.854 & 1.453 \\
\hline $07 / 25 / 00$ & $9: 55: 48$ & 20.4862 & 8.775 & 29.834 & 1.443 \\
\hline $07 / 25 / 00$ & 9:56:03 & 20.7362 & 8.76 & 29.834 & 1.458 \\
\hline $07 / 25 / 00$ & $9: 56: 18$ & 20.9862 & 8.777 & 29.834 & 1.441 \\
\hline $07 / 25 / 00$ & $9: 56: 33$ & 21.2362 & 8.773 & 29.834 & 1.445 \\
\hline $07 / 25 / 00$ & $9: 56: 48$ & 21.4862 & 8.775 & 29.831 & 1.443 \\
\hline $07 / 25 / 00$ & $9: 57: 03$ & 21.7362 & 8.765 & 29.834 & 1.453 \\
\hline $07 / 25 / 00$ & $9: 57: 18$ & 21.9862 & 8.755 & 29.831 & 1.463 \\
\hline $07 / 25 / 00$ & $9: 57: 33$ & 22.2362 & 8.754 & 29.831 & 1.464 \\
\hline $07 / 25 / 00$ & $9: 57: 48$ & 22.4862 & 8.767 & 29.831 & 1.451 \\
\hline $07 / 25 / 00$ & $9: 58: 03$ & 22.7362 & 8.765 & 29.831 & 1.453 \\
\hline $07 / 25 / 00$ & $9: 58: 18$ & 22.9862 & 8.773 & 29.831 & 1.445 \\
\hline $07 / 25 / 00$ & $9: 58: 33$ & 23.2362 & 8.76 & 29.831 & 1.458 \\
\hline $07 / 25 / 00$ & $9: 58: 48$ & 23.4862 & 8.764 & 29.834 & 1.454 \\
\hline $07 / 25 / 00$ & 9:59:03 & 23.7362 & 8.765 & 29.834 & 1.453 \\
\hline $07 / 25 / 00$ & $9: 59: 18$ & 23.9862 & 8.768 & 29.834 & 1.45 \\
\hline $07 / 25 / 00$ & $9: 59: 33$ & 24.2362 & 8.761 & 29.834 & 1.457 \\
\hline $07 / 25 / 00$ & $9: 59: 48$ & 24.4862 & 8.757 & 29.834 & 1.461 \\
\hline $07 / 25 / 00$ & $10: 00: 03$ & 24.7362 & 8.765 & 29.834 & 1.453 \\
\hline $07 / 25 / 00$ & 10:00:18 & 24.9862 & 8.767 & 29.831 & 1.451 \\
\hline $07 / 25 / 00$ & 10:00:33 & 25.2362 & 8.773 & 29.836 & 1.445 \\
\hline $07 / 25 / 00$ & $10: 00: 48$ & 25.4862 & 8.755 & 29.831 & 1.463 \\
\hline $07 / 25 / 00$ & 10:01:03 & 25.7362 & 8.76 & 29.834 & 1.458 \\
\hline $07 / 25 / 00$ & 10:01:18 & 25.9862 & 8.749 & 29.831 & 1.469 \\
\hline $07 / 25 / 00$ & 10:01:33 & 26.2362 & 8.761 & 29.831 & 1.457 \\
\hline $07 / 25 / 00$ & $10: 01: 48$ & 26.4862 & 8.761 & 29.831 & 1.457 \\
\hline $07 / 25 / 00$ & 10:02:03 & 26.7362 & 8.761 & 29.831 & 1.457 \\
\hline $07 / 25 / 00$ & 10:02:18 & 26.9862 & 8.773 & 29.831 & 1.445 \\
\hline $07 / 25 / 00$ & $10: 02: 33$ & 27.2362 & 8.76 & 29.831 & 1.458 \\
\hline
\end{tabular}




\section{Appendix A (continued)}

\begin{tabular}{|c|c|c|c|c|c|}
\hline $07 / 25 / 00$ & 10:02:48 & 27.4862 & 8.754 & 29.852 & 1.464 \\
\hline $07 / 25 / 00$ & 10:03:03 & 27.7362 & 8.77 & 29.836 & 1.448 \\
\hline $07 / 25 / 00$ & 10:03:18 & 27.9862 & 8.757 & 29.834 & 1.461 \\
\hline $07 / 25 / 00$ & 10:03:33 & 28.2362 & 8.764 & 29.834 & 1.454 \\
\hline $07 / 25 / 00$ & 10:03:48 & 28.4862 & 8.774 & 29.836 & 1.444 \\
\hline $07 / 25 / 00$ & 10:04:03 & 28.7362 & 8.755 & 29.834 & 1.463 \\
\hline $07 / 25 / 00$ & 10:04:18 & 28.9862 & 8.762 & 29.834 & 1.456 \\
\hline $07 / 25 / 00$ & 10:04:33 & 29.2362 & 8.764 & 29.831 & 1.454 \\
\hline $07 / 25 / 00$ & 10:04:48 & 29.4862 & 8.752 & 29.831 & 1.466 \\
\hline $07 / 25 / 00$ & $10: 05: 03$ & 29.7362 & 8.762 & 29.831 & 1.456 \\
\hline $07 / 25 / 00$ & $10: 05: 18$ & 29.9862 & 8.76 & 29.831 & 1.458 \\
\hline $07 / 25 / 00$ & 10:05:33 & 30.2362 & 8.76 & 29.831 & 1.458 \\
\hline $07 / 25 / 00$ & 10:05:48 & 30.4862 & 8.762 & 29.831 & 1.456 \\
\hline $07 / 25 / 00$ & 10:06:03 & 30.7362 & 8.768 & 29.834 & 1.45 \\
\hline $07 / 25 / 00$ & 10:06:18 & 30.9862 & 8.757 & 29.834 & 1.461 \\
\hline 07/25/00 & 10:06:33 & 31.2362 & 8.76 & 29.834 & 1.458 \\
\hline $07 / 25 / 00$ & 10:06:48 & 31.4862 & 8.751 & 29.836 & 1.467 \\
\hline $07 / 25 / 00$ & 10:07:03 & 31.7362 & 8.755 & 29.834 & 1.463 \\
\hline 07/25/00 & 10:07:18 & 31.9862 & 8.755 & 29.834 & 1.463 \\
\hline $07 / 25 / 00$ & 10:07:33 & 32.2362 & 8.771 & 29.836 & 1.447 \\
\hline $07 / 25 / 00$ & 10:07:48 & 32.4862 & 8.761 & 29.834 & 1.457 \\
\hline $07 / 25 / 00$ & 10:08:03 & 32.7362 & 8.754 & 29.834 & 1.464 \\
\hline $07 / 25 / 00$ & 10:08:18 & 32.9862 & 8.726 & 29.836 & 1.492 \\
\hline 07/25/00 & 10:08:33 & 33.2362 & 8.744 & 29.836 & 1.474 \\
\hline $07 / 25 / 00$ & 10:08:48 & 33.4862 & 8.752 & 29.834 & 1.466 \\
\hline $07 / 25 / 00$ & 10:09:03 & 33.7362 & 8.755 & 29.834 & 1.463 \\
\hline 07/25/00 & 10:09:18 & 33.9862 & 8.752 & 29.834 & 1.466 \\
\hline $07 / 25 / 00$ & 10:09:33 & 34.2362 & 8.785 & 29.834 & 1.433 \\
\hline $07 / 25 / 00$ & 10:09:48 & 34.4862 & 8.76 & 29.834 & 1.458 \\
\hline $07 / 25 / 00$ & 10:10:03 & 34.7362 & 8.754 & 29.834 & 1.464 \\
\hline $07 / 25 / 00$ & 10:10:18 & 34.9862 & 8.741 & 29.834 & 1.477 \\
\hline $07 / 25 / 00$ & 10:10:33 & 35.2362 & 8.748 & 29.834 & 1.47 \\
\hline $07 / 25 / 00$ & 10:10:48 & 35.4862 & 8.755 & 29.836 & 1.463 \\
\hline $07 / 25 / 00$ & 10:11:03 & 35.7362 & 8.747 & 29.834 & 1.471 \\
\hline $07 / 25 / 00$ & 10:11:18 & 35.9862 & 8.742 & 29.834 & 1.476 \\
\hline 07/25/00 & 10:11:33 & 36.2362 & 8.722 & 29.834 & 1.496 \\
\hline $07 / 25 / 00$ & 10:11:48 & 36.4862 & 8.739 & 29.834 & 1.479 \\
\hline $07 / 25 / 00$ & 10:12:03 & 36.7362 & 8.741 & 29.834 & 1.477 \\
\hline $07 / 25 / 00$ & 10:12:18 & 36.9862 & 8.765 & 29.834 & 1.453 \\
\hline $07 / 25 / 00$ & 10:12:33 & 37.2362 & 8.749 & 29.834 & 1.469 \\
\hline $07 / 25 / 00$ & 10:12:48 & 37.4862 & 8.744 & 29.834 & 1.474 \\
\hline $07 / 25 / 00$ & 10:13:03 & 37.7362 & 8.741 & 29.834 & 1.477 \\
\hline 07/25/00 & 10:13:18 & 37.9862 & 8.754 & 29.831 & 1.464 \\
\hline $07 / 25 / 00$ & 10:13:33 & 38.2362 & 8.757 & 29.834 & 1.461 \\
\hline $07 / 25 / 00$ & 10:13:48 & 38.4862 & 8.741 & 29.831 & 1.477 \\
\hline $07 / 25 / 00$ & 10:14:03 & 38.7362 & 8.742 & 29.831 & 1.476 \\
\hline $07 / 25 / 00$ & 10:14:18 & 38.9862 & 8.742 & 29.834 & 1.476 \\
\hline $07 / 25 / 00$ & 10:14:33 & 39.2362 & 8.737 & 29.831 & 1.481 \\
\hline $07 / 25 / 00$ & 10:14:48 & 39.4862 & 8.749 & 29.834 & 1.469 \\
\hline $07 / 25 / 00$ & 10:15:03 & 39.7362 & 8.739 & 29.834 & 1.479 \\
\hline $07 / 25 / 00$ & 10:15:18 & 39.9862 & 8.738 & 29.834 & 1.4 \\
\hline
\end{tabular}




\section{Appendix A (continued)}

\begin{tabular}{|c|c|c|c|c|c|}
\hline $07 / 25 / 00$ & 10:15:33 & 40.2362 & 8.744 & 29.834 & 1.474 \\
\hline $07 / 25 / 00$ & 10:15:48 & 40.4862 & 8.752 & 29.831 & 1.466 \\
\hline $07 / 25 / 00$ & 10:16:03 & 40.7362 & 8.744 & 29.834 & 1.474 \\
\hline $07 / 25 / 00$ & $10: 16: 18$ & 40.9862 & 8.686 & 29.831 & 1.532 \\
\hline $07 / 25 / 00$ & 10:16:33 & 41.2362 & 8.725 & 29.834 & 1.493 \\
\hline $07 / 25 / 00$ & $10: 16: 48$ & 41.4862 & 8.732 & 29.834 & 1.486 \\
\hline $07 / 25 / 00$ & $10: 17: 03$ & 41.7362 & 8.784 & 29.834 & 1.434 \\
\hline $07 / 25 / 00$ & 10:17:18 & 41.9862 & 8.749 & 29.834 & 1.469 \\
\hline $07 / 25 / 00$ & 10:17:33 & 42.2362 & 8.755 & 29.831 & 1.463 \\
\hline $07 / 25 / 00$ & $10: 17: 48$ & 42.4862 & 8.742 & 29.834 & 1.476 \\
\hline $07 / 25 / 00$ & 10:18:03 & 42.7362 & 8.742 & 29.834 & 1.476 \\
\hline $07 / 25 / 00$ & 10:18:18 & 42.9862 & 8.739 & 29.834 & 1.479 \\
\hline $07 / 25 / 00$ & 10:18:33 & 43.2362 & 8.737 & 29.834 & 1.481 \\
\hline $07 / 25 / 00$ & 10:18:48 & 43.4862 & 8.741 & 29.834 & 1.477 \\
\hline $07 / 25 / 00$ & 10:19:03 & 43.7362 & 8.732 & 29.834 & 1.486 \\
\hline $07 / 25 / 00$ & 10:19:18 & 43.9862 & 8.747 & 29.834 & 1.471 \\
\hline $07 / 25 / 00$ & 10:19:33 & 44.2362 & 8.738 & 29.831 & 1.48 \\
\hline $07 / 25 / 00$ & 10:19:48 & 44.4862 & 8.739 & 29.831 & 1.479 \\
\hline $07 / 25 / 00$ & $10: 20: 03$ & 44.7362 & 8.747 & 29.831 & 1.471 \\
\hline $07 / 25 / 00$ & 10:20:18 & 44.9862 & 8.728 & 29.831 & 1.49 \\
\hline $07 / 25 / 00$ & 10:20:33 & 45.2362 & 8.696 & 29.831 & 1.522 \\
\hline $07 / 25 / 00$ & $10: 20: 48$ & 45.4862 & 8.765 & 29.831 & 1.453 \\
\hline $07 / 25 / 00$ & $10: 21: 03$ & 45.7362 & 8.745 & 29.831 & 1.473 \\
\hline $07 / 25 / 00$ & $10: 21: 18$ & 45.9862 & 8.737 & 29.834 & 1.481 \\
\hline $07 / 25 / 00$ & 10:21:33 & 46.2362 & 8.741 & 29.846 & 1.477 \\
\hline $07 / 25 / 00$ & $10: 21: 48$ & 46.4862 & 8.741 & 29.838 & 1.477 \\
\hline $07 / 25 / 00$ & 10:22:03 & 46.7362 & 8.738 & 29.834 & 1.48 \\
\hline $07 / 25 / 00$ & 10:22:18 & 46.9862 & 8.741 & 29.834 & 1.477 \\
\hline $07 / 25 / 00$ & 10:22:33 & 47.2362 & 8.68 & 29.834 & 1.538 \\
\hline $07 / 25 / 00$ & 10:22:48 & 47.4862 & 8.768 & 29.834 & 1.45 \\
\hline $07 / 25 / 00$ & $10: 23: 03$ & 47.7362 & 8.765 & 29.834 & 1.453 \\
\hline $07 / 25 / 00$ & 10:23:18 & 47.9862 & 8.742 & 29.834 & 1.476 \\
\hline $07 / 25 / 00$ & 10:23:33 & 48.2362 & 8.734 & 29.831 & 1.484 \\
\hline $07 / 25 / 00$ & 10:23:48 & 48.4862 & 8.737 & 29.831 & 1.481 \\
\hline $07 / 25 / 00$ & $10: 24: 03$ & 48.7362 & 8.748 & 29.831 & 1.47 \\
\hline $07 / 25 / 00$ & $10: 24: 18$ & 48.9862 & 8.735 & 29.831 & 1.483 \\
\hline $07 / 25 / 00$ & 10:24:33 & 49.2362 & 8.696 & 29.831 & 1.522 \\
\hline $07 / 25 / 00$ & $10: 24: 48$ & 49.4862 & 8.771 & 29.834 & 1.447 \\
\hline $07 / 25 / 00$ & $10: 25: 03$ & 49.7362 & 8.745 & 29.831 & 1.473 \\
\hline $07 / 25 / 00$ & 10:25:18 & 49.9862 & 8.734 & 29.831 & 1.484 \\
\hline $07 / 25 / 00$ & $10: 25: 33$ & 50.2362 & 8.738 & 29.831 & 1.48 \\
\hline $07 / 25 / 00$ & 10:25:48 & 50.4862 & 8.734 & 29.831 & 1.484 \\
\hline $07 / 25 / 00$ & $10: 26: 03$ & 50.7362 & 8.737 & 29.834 & 1.481 \\
\hline $07 / 25 / 00$ & 10:26:18 & 50.9862 & 8.738 & 29.831 & 1.48 \\
\hline $07 / 25 / 00$ & 10:26:33 & 51.2362 & 8.66 & 29.834 & 1.558 \\
\hline $07 / 25 / 00$ & $10: 26: 48$ & 51.4862 & 8.729 & 29.834 & 1.489 \\
\hline $07 / 25 / 00$ & $10: 27: 03$ & 51.7362 & 8.735 & 29.834 & 1.483 \\
\hline $07 / 25 / 00$ & 10:27:18 & 51.9862 & 8.738 & 29.834 & 1.48 \\
\hline $07 / 25 / 00$ & $10: 27: 33$ & 52.2362 & 8.729 & 29.84 & 1.489 \\
\hline $07 / 25 / 00$ & $10: 27: 48$ & 52.4862 & 8.738 & 29.842 & 1.48 \\
\hline $07 / 25 / 00$ & $10: 28: 03$ & 52.7362 & 8.722 & 29.836 & 1.496 \\
\hline
\end{tabular}




\section{Appendix A (continued)}

\begin{tabular}{|c|c|c|c|c|c|}
\hline $07 / 25 / 00$ & $10: 28: 18$ & 52.9862 & 8.728 & 29.838 & 1.49 \\
\hline 07/25/00 & 10:28:33 & 53.2362 & 8.726 & 29.836 & 1.492 \\
\hline 07/25/00 & 10:28:48 & 53.4862 & 8.719 & 29.836 & 1.499 \\
\hline 07/25/00 & 10:29:03 & 53.7362 & 8.742 & 29.836 & 1.476 \\
\hline 07/25/00 & 10:29:18 & 53.9862 & 8.744 & 29.836 & 1.474 \\
\hline 07/25/00 & 10:29:33 & 54.2362 & 8.737 & 29.836 & 1.481 \\
\hline 07/25/00 & 10:29:48 & 54.4862 & 8.726 & 29.836 & 1.492 \\
\hline 07/25/00 & 10:30:03 & 54.7362 & 8.729 & 29.836 & 1.489 \\
\hline 07/25/00 & 10:30:18 & 54.9862 & 8.738 & 29.836 & 1.48 \\
\hline 07/25/00 & 10:30:33 & 55.2362 & 8.729 & 29.836 & 1.489 \\
\hline $07 / 25 / 00$ & 10:30:48 & 55.4862 & 8.731 & 29.836 & 1.487 \\
\hline 07/25/00 & 10:31:03 & 55.7362 & 8.724 & 29.836 & 1.494 \\
\hline 07/25/00 & 10:31:18 & 55.9862 & 8.742 & 29.836 & 1.476 \\
\hline 07/25/00 & 10:31:33 & 56.2362 & 8.698 & 29.836 & 1.52 \\
\hline $07 / 25 / 00$ & 10:31:48 & 56.4862 & 8.745 & 29.836 & 1.473 \\
\hline 07/25/00 & 10:32:03 & 56.7362 & 8.741 & 29.836 & 1.477 \\
\hline 07/25/00 & 10:32:18 & 56.9862 & 8.737 & 29.836 & 1.481 \\
\hline 07/25/00 & 10:32:33 & 57.2362 & 8.734 & 29.836 & 1.484 \\
\hline 07/25/00 & 10:32:48 & 57.4862 & 8.724 & 29.836 & 1.494 \\
\hline 07/25/00 & 10:33:03 & 57.7362 & 8.737 & 29.838 & 1.481 \\
\hline 07/25/00 & 10:33:18 & 57.9862 & 8.738 & 29.836 & 1.48 \\
\hline 07/25/00 & 10:33:33 & 58.2362 & 8.734 & 29.836 & 1.484 \\
\hline $07 / 25 / 00$ & 10:33:48 & 58.4862 & 8.732 & 29.838 & 1.486 \\
\hline 07/25/00 & 10:34:03 & 58.7362 & 8.732 & 29.838 & 1.486 \\
\hline 07/25/00 & 10:34:18 & 58.9862 & 8.725 & 29.838 & 1.493 \\
\hline 07/25/00 & 10:34:33 & 59.2362 & 8.679 & 29.838 & 1.539 \\
\hline 07/25/00 & $10: 34: 48$ & 59.4862 & 8.739 & 29.838 & 1.479 \\
\hline 07/25/00 & 10:35:03 & 59.7362 & 8.729 & 29.838 & 1.489 \\
\hline 07/25/00 & 10:35:18 & 59.9862 & 8.725 & 29.836 & 1.493 \\
\hline 07/25/00 & 10:35:33 & 60.2362 & 8.724 & 29.838 & 1.494 \\
\hline 07/25/00 & 10:35:48 & 60.4862 & 8.734 & 29.838 & 1.484 \\
\hline 07/25/00 & 10:36:03 & 60.7362 & 8.725 & 29.838 & 1.493 \\
\hline 07/25/00 & 10:36:18 & 60.9862 & 8.732 & 29.838 & 1.486 \\
\hline 07/25/00 & 10:36:33 & 61.2362 & 8.748 & 29.838 & 1.47 \\
\hline 07/25/00 & $10: 36: 48$ & 61.4862 & 8.724 & 29.838 & 1.494 \\
\hline 07/25/00 & 10:37:03 & 61.7362 & 8.732 & 29.854 & 1.486 \\
\hline 07/25/00 & 10:37:18 & 61.9862 & 8.725 & 29.846 & 1.493 \\
\hline 07/25/00 & 10:37:33 & 62.2362 & 8.725 & 29.842 & 1.493 \\
\hline 07/25/00 & 10:37:48 & 62.4862 & 8.718 & 29.84 & 1.5 \\
\hline $07 / 25 / 00$ & 10:38:03 & 62.7362 & 8.724 & 29.838 & 1.494 \\
\hline 07/25/00 & 10:38:18 & 62.9862 & 8.724 & 29.84 & 1.494 \\
\hline 07/25/00 & 10:38:33 & 63.2362 & 8.675 & 29.838 & 1.543 \\
\hline 07/25/00 & 10:38:48 & 63.4862 & 8.742 & 29.838 & 1.476 \\
\hline 07/25/00 & 10:39:03 & 63.7362 & 8.728 & 29.84 & 1.49 \\
\hline 07/25/00 & 10:39:18 & 63.9862 & 8.728 & 29.84 & 1.49 \\
\hline 07/25/00 & 10:39:33 & 64.2362 & 8.724 & 29.838 & 1.494 \\
\hline $07 / 25 / 00$ & 10:39:48 & 64.4862 & 8.718 & 29.838 & 1.5 \\
\hline 07/25/00 & 10:40:03 & 64.7362 & 8.663 & 29.838 & 1.555 \\
\hline 07/25/00 & 10:40:18 & 64.9862 & 8.735 & 29.84 & 1.483 \\
\hline 07/25/00 & 10:40:33 & 65.2362 & 8.721 & 29.84 & 1.497 \\
\hline 07/25/00 & 10:40:48 & 65.4862 & 8.729 & 29.84 & 1.48 \\
\hline
\end{tabular}




\section{Appendix A (continued)}

\begin{tabular}{|c|c|c|c|c|c|}
\hline 07/25/00 & 10:41:03 & 65.7362 & 8.718 & 29.836 & 1.5 \\
\hline 07/25/00 & 10:41:18 & 65.9862 & 8.713 & 29.84 & 1.505 \\
\hline 07/25/00 & 10:41:33 & 66.2362 & 8.718 & 29.838 & 1.5 \\
\hline 07/25/00 & 10:41:48 & 66.4862 & 8.64 & 29.84 & 1.578 \\
\hline 07/25/00 & 10:42:03 & 66.7362 & 8.745 & 29.838 & 1.473 \\
\hline 07/25/00 & 10:42:18 & 66.9862 & 8.729 & 29.838 & 1.489 \\
\hline 07/25/00 & 10:42:33 & 67.2362 & 8.718 & 29.838 & 1.5 \\
\hline 07/25/00 & 10:42:48 & 67.4862 & 8.718 & 29.84 & 1.5 \\
\hline 07/25/00 & 10:43:03 & 67.7362 & 8.718 & 29.84 & 1.5 \\
\hline $07 / 25 / 00$ & 10:43:18 & 67.9862 & 8.718 & 29.84 & 1.5 \\
\hline 07/25/00 & 10:43:33 & 68.2362 & 8.677 & 29.84 & 1.541 \\
\hline 07/25/00 & 10:43:48 & 68.4862 & 8.732 & 29.84 & 1.486 \\
\hline 07/25/00 & 10:44:03 & 68.7362 & 8.725 & 29.84 & 1.493 \\
\hline 07/25/00 & 10:44:18 & 68.9862 & 8.706 & 29.84 & 1.512 \\
\hline 07/25/00 & 10:44:33 & 69.2362 & 8.712 & 29.84 & 1.506 \\
\hline 07/25/00 & 10:44:48 & 69.4862 & 8.718 & 29.84 & 1.5 \\
\hline 07/25/00 & 10:45:03 & 69.7362 & 8.713 & 29.84 & 1.505 \\
\hline 07/25/00 & 10:45:18 & 69.9862 & 8.709 & 29.84 & 1.509 \\
\hline 07/25/00 & 10:45:33 & 70.2362 & 8.705 & 29.84 & 1.513 \\
\hline 07/25/00 & 10:45:48 & 70.4862 & 8.712 & 29.85 & 1.506 \\
\hline 07/25/00 & 10:46:03 & 70.7362 & 8.68 & 29.844 & 1.538 \\
\hline $07 / 25 / 00$ & 10:46:18 & 70.9862 & 8.742 & 29.842 & 1.476 \\
\hline 07/25/00 & 10:46:33 & 71.2362 & 8.724 & 29.842 & 1.494 \\
\hline 07/25/00 & 10:46:48 & 71.4862 & 8.706 & 29.842 & 1.512 \\
\hline 07/25/00 & 10:47:03 & 71.7362 & 8.716 & 29.84 & 1.502 \\
\hline 07/25/00 & 10:47:18 & 71.9862 & 8.696 & 29.84 & 1.522 \\
\hline 07/25/00 & 10:47:33 & 72.2362 & 8.706 & 29.84 & 1.512 \\
\hline 07/25/00 & 10:47:48 & 72.4862 & 8.708 & 29.842 & 1.51 \\
\hline 07/25/00 & 10:48:03 & 72.7362 & 8.701 & 29.842 & 1.517 \\
\hline 07/25/00 & 10:48:18 & 72.9862 & 8.705 & 29.84 & 1.513 \\
\hline 07/25/00 & 10:48:33 & 73.2362 & 8.702 & 29.842 & 1.516 \\
\hline 07/25/00 & 10:48:48 & 73.4862 & 8.725 & 29.84 & 1.493 \\
\hline 07/25/00 & 10:49:03 & 73.7362 & 8.711 & 29.842 & 1.507 \\
\hline $07 / 25 / 00$ & 10:49:18 & 73.9862 & 8.709 & 29.842 & 1.509 \\
\hline 07/25/00 & 10:49:33 & 74.2362 & 8.709 & 29.84 & 1.509 \\
\hline 07/25/00 & 10:49:48 & 74.4862 & 8.702 & 29.84 & 1.516 \\
\hline 07/25/00 & $10: 50: 03$ & 74.7362 & 8.703 & 29.842 & 1.515 \\
\hline 07/25/00 & 10:50:18 & 74.9862 & 8.709 & 29.84 & 1.509 \\
\hline 07/25/00 & 10:50:33 & 75.2362 & 8.685 & 29.84 & 1.533 \\
\hline 07/25/00 & 10:50:48 & 75.4862 & 8.724 & 29.842 & 1.494 \\
\hline 07/25/00 & 10:51:03 & 75.7362 & 8.701 & 29.84 & 1.517 \\
\hline 07/25/00 & 10:51:18 & 75.9862 & 8.695 & 29.84 & 1.523 \\
\hline 07/25/00 & 10:51:33 & 76.2362 & 8.696 & 29.84 & 1.522 \\
\hline 07/25/00 & 10:51:48 & 76.4862 & 8.696 & 29.838 & 1.522 \\
\hline 07/25/00 & 10:52:03 & 76.7362 & 8.693 & 29.84 & 1.525 \\
\hline $07 / 25 / 00$ & 10:52:18 & 76.9862 & 8.701 & 29.84 & 1.517 \\
\hline 07/25/00 & 10:52:33 & 77.2362 & 8.696 & 29.842 & 1.522 \\
\hline 07/25/00 & 10:52:48 & 77.4862 & 8.686 & 29.84 & 1.532 \\
\hline 07/25/00 & 10:53:03 & 77.7362 & 8.751 & 29.84 & 1.467 \\
\hline 07/25/00 & 10:53:18 & 77.9862 & 8.718 & 29.842 & 1.5 \\
\hline 07/25/00 & 10:53:33 & 78.2362 & 8.706 & 29.84 & 1.512 \\
\hline
\end{tabular}




\section{Appendix A (continued)}

\begin{tabular}{|c|c|c|c|c|c|}
\hline $07 / 25 / 00$ & $10: 53: 48$ & 78.4862 & 8.702 & 29.84 & 1.516 \\
\hline $07 / 25 / 00$ & $10: 54: 03$ & 78.7362 & 8.696 & 29.84 & 1.522 \\
\hline $07 / 25 / 00$ & $10: 54: 18$ & 78.9862 & 8.706 & 29.842 & 1.512 \\
\hline $07 / 25 / 00$ & $10: 54: 33$ & 79.2362 & 8.689 & 29.842 & 1.529 \\
\hline $07 / 25 / 00$ & $10: 54: 48$ & 79.4862 & 8.716 & 29.84 & 1.502 \\
\hline 07/25/00 & $10: 55: 03$ & 79.7362 & 8.724 & 29.84 & 1.494 \\
\hline $07 / 25 / 00$ & $10: 55: 18$ & 79.9862 & 8.709 & 29.84 & 1.509 \\
\hline $07 / 25 / 00$ & $10: 55: 33$ & 80.2362 & 8.708 & 29.84 & 1.51 \\
\hline $07 / 25 / 00$ & $10: 55: 48$ & 80.4862 & 8.709 & 29.84 & 1.509 \\
\hline $07 / 25 / 00$ & $10: 56: 03$ & 80.7362 & 8.701 & 29.842 & 1.517 \\
\hline $07 / 25 / 00$ & $10: 56: 18$ & 80.9862 & 8.662 & 29.84 & 1.556 \\
\hline $07 / 25 / 00$ & $10: 56: 33$ & 81.2362 & 8.751 & 29.84 & 1.467 \\
\hline $07 / 25 / 00$ & $10: 56: 48$ & 81.4862 & 8.719 & 29.84 & 1.499 \\
\hline 07/25/00 & $10: 57: 03$ & 81.7362 & 8.711 & 29.84 & 1.507 \\
\hline 07/25/00 & $10: 57: 18$ & 81.9862 & 8.705 & 29.842 & 1.513 \\
\hline $07 / 25 / 00$ & $10: 57: 33$ & 82.2362 & 8.698 & 29.842 & 1.52 \\
\hline 07/25/00 & $10: 57: 48$ & 82.4862 & 8.703 & 29.84 & 1.515 \\
\hline 07/25/00 & 10:58:03 & 82.7362 & 8.702 & 29.842 & 1.516 \\
\hline $07 / 25 / 00$ & 10:58:18 & 82.9862 & 8.702 & 29.842 & 1.516 \\
\hline $07 / 25 / 00$ & $10: 58: 33$ & 83.2362 & 8.706 & 29.842 & 1.512 \\
\hline $07 / 25 / 00$ & $10: 58: 48$ & 83.4862 & 8.696 & 29.842 & 1.522 \\
\hline $07 / 25 / 00$ & $10: 59: 03$ & 83.7362 & 8.686 & 29.842 & 1.532 \\
\hline $07 / 25 / 00$ & $10: 59: 18$ & 83.9862 & 8.719 & 29.842 & 1.499 \\
\hline $07 / 25 / 00$ & $10: 59: 33$ & 84.2362 & 8.705 & 29.844 & 1.513 \\
\hline $07 / 25 / 00$ & $10: 59: 48$ & 84.4862 & 8.705 & 29.844 & 1.513 \\
\hline 07/25/00 & 11:00:03 & 84.7362 & 8.699 & 29.842 & 1.519 \\
\hline $07 / 25 / 00$ & 11:00:18 & 84.9862 & 8.703 & 29.842 & 1.515 \\
\hline $07 / 25 / 00$ & $11: 00: 33$ & 85.2362 & 8.695 & 29.842 & 1.523 \\
\hline $07 / 25 / 00$ & $11: 00: 48$ & 85.4862 & 8.702 & 29.842 & 1.516 \\
\hline $07 / 25 / 00$ & 11:01:03 & 85.7362 & 8.701 & 29.842 & 1.517 \\
\hline $07 / 25 / 00$ & 11:01:18 & 85.9862 & 8.692 & 29.842 & 1.526 \\
\hline $07 / 25 / 00$ & 11:01:33 & 86.2362 & 8.703 & 29.842 & 1.515 \\
\hline 07/25/00 & 11:01:48 & 86.4862 & 8.696 & 29.842 & 1.522 \\
\hline $07 / 25 / 00$ & 11:02:03 & 86.7362 & 8.676 & 29.842 & 1.542 \\
\hline $07 / 25 / 00$ & 11:02:18 & 86.9862 & 8.728 & 29.842 & 1.49 \\
\hline 07/25/00 & 11:02:33 & 87.2362 & 8.699 & 29.844 & 1.519 \\
\hline $07 / 25 / 00$ & 11:02:48 & 87.4862 & 8.692 & 29.844 & 1.526 \\
\hline 07/25/00 & 11:03:03 & 87.7362 & 8.685 & 29.844 & 1.533 \\
\hline 07/25/00 & 11:03:18 & 87.9862 & 8.689 & 29.842 & 1.529 \\
\hline $07 / 25 / 00$ & 11:03:33 & 88.2362 & 8.695 & 29.842 & 1.523 \\
\hline $07 / 25 / 00$ & 11:03:48 & 88.4862 & 8.695 & 29.842 & 1.523 \\
\hline 07/25/00 & 11:04:03 & 88.7362 & 8.696 & 29.842 & 1.522 \\
\hline $07 / 25 / 00$ & 11:04:18 & 88.9862 & 8.656 & 29.842 & 1.562 \\
\hline $07 / 25 / 00$ & 11:04:33 & 89.2362 & 8.728 & 29.842 & 1.49 \\
\hline $07 / 25 / 00$ & 11:04:48 & 89.4862 & 8.706 & 29.844 & 1.512 \\
\hline $07 / 25 / 00$ & 11:05:03 & 89.7362 & 8.696 & 29.844 & 1.522 \\
\hline 07/25/00 & 11:05:18 & 89.9862 & 8.708 & 29.844 & 1.51 \\
\hline $07 / 25 / 00$ & 11:05:33 & 90.2362 & 8.701 & 29.844 & 1.517 \\
\hline $07 / 25 / 00$ & 11:05:48 & 90.4862 & 8.695 & 29.844 & 1.523 \\
\hline $07 / 25 / 00$ & 11:06:03 & 90.7362 & 8.695 & 29.844 & 1.523 \\
\hline 07/25/00 & 11:06:18 & 90.9862 & 8.702 & 29.844 & 1.516 \\
\hline
\end{tabular}




\section{Appendix A (continued)}

\begin{tabular}{|c|c|c|c|c|c|}
\hline 07/25/00 & 11:06:33 & 91.2362 & 8.656 & 29.844 & 1.562 \\
\hline 07/25/00 & $11: 06: 48$ & 91.4862 & 8.726 & 29.844 & 1.492 \\
\hline 07/25/00 & 11:07:03 & 91.7362 & 8.703 & 29.844 & 1.515 \\
\hline 07/25/00 & 11:07:18 & 91.9862 & 8.693 & 29.844 & 1.525 \\
\hline 07/25/00 & 11:07:33 & 92.2362 & 8.698 & 29.844 & 1.52 \\
\hline 07/25/00 & 11:07:48 & 92.4862 & 8.693 & 29.844 & 1.525 \\
\hline 07/25/00 & 11:08:03 & 92.7362 & 8.702 & 29.844 & 1.516 \\
\hline 07/25/00 & 11:08:18 & 92.9862 & 8.695 & 29.844 & 1.523 \\
\hline 07/25/00 & 11:08:33 & 93.2362 & 8.695 & 29.862 & 1.523 \\
\hline 07/25/00 & $11: 08: 48$ & 93.4862 & 8.686 & 29.85 & 1.532 \\
\hline 07/25/00 & 11:09:03 & 93.7362 & 8.667 & 29.846 & 1.551 \\
\hline 07/25/00 & 11:09:18 & 93.9862 & 8.716 & 29.846 & 1.502 \\
\hline 07/25/00 & 11:09:33 & 94.2362 & 8.698 & 29.846 & 1.52 \\
\hline 07/25/00 & 11:09:48 & 94.4862 & 8.679 & 29.846 & 1.539 \\
\hline 07/25/00 & 11:10:03 & 94.7362 & 8.682 & 29.844 & 1.536 \\
\hline 07/25/00 & 11:10:18 & 94.9862 & 8.688 & 29.844 & 1.53 \\
\hline 07/25/00 & 11:10:33 & 95.2362 & 8.676 & 29.844 & 1.542 \\
\hline 07/25/00 & 11:10:48 & 95.4862 & 8.676 & 29.842 & 1.542 \\
\hline 07/25/00 & 11:11:03 & 95.7362 & 8.688 & 29.844 & 1.53 \\
\hline 07/25/00 & 11:11:18 & 95.9862 & 8.644 & 29.842 & 1.574 \\
\hline 07/25/00 & $11: 11: 33$ & 96.2362 & 8.721 & 29.842 & 1.497 \\
\hline 07/25/00 & $11: 11: 48$ & 96.4862 & 8.703 & 29.844 & 1.515 \\
\hline 07/25/00 & 11:12:03 & 96.7362 & 8.69 & 29.842 & 1.528 \\
\hline 07/25/00 & 11:12:18 & 96.9862 & 8.688 & 29.844 & 1.53 \\
\hline 07/25/00 & 11:12:33 & 97.2362 & 8.692 & 29.844 & 1.526 \\
\hline 07/25/00 & 11:12:48 & 97.4862 & 8.685 & 29.844 & 1.533 \\
\hline 07/25/00 & 11:13:03 & 97.7362 & 8.679 & 29.842 & 1.539 \\
\hline 07/25/00 & 11:13:18 & 97.9862 & 8.683 & 29.844 & 1.535 \\
\hline 07/25/00 & 11:13:33 & 98.2362 & 8.69 & 29.844 & 1.528 \\
\hline 07/25/00 & 11:13:48 & 98.4862 & 8.688 & 29.844 & 1.53 \\
\hline 07/25/00 & 11:14:03 & 98.7362 & 8.713 & 29.842 & 1.505 \\
\hline 07/25/00 & 11:14:18 & 98.9862 & 8.685 & 29.842 & 1.533 \\
\hline 07/25/00 & $11: 14: 33$ & 99.2362 & 8.662 & 29.842 & 1.556 \\
\hline 07/25/00 & $11: 14: 48$ & 99.4862 & 8.676 & 29.844 & 1.542 \\
\hline 07/25/00 & 11:15:03 & 99.7362 & 8.666 & 29.844 & 1.552 \\
\hline 07/25/00 & 11:15:18 & 99.9862 & 8.657 & 29.842 & 1.561 \\
\hline 07/25/00 & 11:15:33 & 100.2362 & 8.659 & 29.844 & 1.559 \\
\hline 07/25/00 & 11:15:48 & 100.4862 & 8.663 & 29.842 & 1.555 \\
\hline 07/25/00 & 11:16:03 & 100.7362 & 8.666 & 29.842 & 1.552 \\
\hline 07/25/00 & $11: 16: 18$ & 100.9862 & 8.666 & 29.844 & 1.552 \\
\hline 07/25/00 & 11:16:33 & 101.2362 & 8.666 & 29.842 & 1.552 \\
\hline 07/25/00 & 11:16:48 & 101.4862 & 8.657 & 29.844 & 1.561 \\
\hline 07/25/00 & 11:17:03 & 101.7362 & 8.731 & 29.844 & 1.487 \\
\hline 07/25/00 & 11:17:18 & 101.9862 & 8.703 & 29.844 & 1.515 \\
\hline 07/25/00 & $11: 17: 33$ & 102.2362 & 8.696 & 29.844 & 1.522 \\
\hline 07/25/00 & $11: 17: 48$ & 102.4862 & 8.695 & 29.844 & 1.523 \\
\hline 07/25/00 & 11:18:03 & 102.7362 & 8.683 & 29.844 & 1.535 \\
\hline 07/25/00 & 11:18:18 & 102.9862 & 8.693 & 29.844 & 1.525 \\
\hline 07/25/00 & 11:18:33 & 103.2362 & 8.683 & 29.842 & 1.535 \\
\hline 07/25/00 & 11:18:48 & 103.4862 & 8.693 & 29.844 & 1.525 \\
\hline 07/25/00 & 11:19:03 & 103.7362 & 8.685 & 29.844 & 1.533 \\
\hline
\end{tabular}




\section{Appendix A (continued)}

\begin{tabular}{|c|c|c|c|c|c|}
\hline $07 / 25 / 00$ & $11: 19: 18$ & 103.9862 & 8.69 & 29.844 & $1.52 \varepsilon$ \\
\hline $07 / 25 / 00$ & $11: 19: 33$ & 104.2362 & 8.689 & 29.844 & 1.52 \\
\hline 07/25/00 & $11: 19: 48$ & 104.4862 & 8.689 & 29.842 & 1.52 \\
\hline $07 / 25 / 00$ & $11: 20: 03$ & 104.7362 & 8.631 & 29.844 & 1.58 \\
\hline $07 / 25 / 00$ & $11: 20: 18$ & 104.9862 & 8.682 & 29.844 & 1.536 \\
\hline 07/25/00 & $11: 20: 33$ & 105.2362 & 8.702 & 29.844 & 1.5 \\
\hline $07 / 25 / 00$ & $11: 20: 48$ & 105.4862 & 8.693 & 29.846 & 1.5 \\
\hline $07 / 25 / 00$ & $11: 21: 03$ & 105.7362 & 8.701 & 29.844 & 1 \\
\hline $07 / 25 / 00$ & $11: 21: 18$ & 105.9862 & 8.699 & 29.842 & 1 \\
\hline $07 / 25 / 00$ & $11: 21: 33$ & 106.2362 & 8.698 & 29.844 & \\
\hline $07 / 25 / 00$ & $11: 21: 48$ & 106.4862 & 8.696 & 29.844 & 1.5 \\
\hline 07/25/00 & $11: 22: 03$ & 106.7362 & 8.689 & 29.844 & 1.5 \\
\hline $07 / 25 / 00$ & $11: 22: 18$ & 106.9862 & 8.695 & 29.844 & 1.5 \\
\hline $07 / 25 / 00$ & $11: 22: 33$ & 107.2362 & 8.695 & 29.844 & 1.5 \\
\hline $07 / 25 / 00$ & $11: 22: 48$ & 107.4862 & 8.662 & 29.846 & 1.55 \\
\hline $07 / 25 / 00$ & $11: 23: 03$ & 107.7362 & 8.713 & 29.846 & 1 \\
\hline $07 / 25 / 00$ & $11: 23: 18$ & 107.9862 & 8.695 & 29.846 & 1.52 \\
\hline $07 / 25 / 00$ & $11: 23: 33$ & 108.2362 & 8.699 & 29.846 & $1.51 \mathrm{C}$ \\
\hline $07 / 25 / 00$ & $11: 23: 48$ & 108.4862 & 8.689 & 29.846 & 1.5 \\
\hline $07 / 25 / 00$ & $11: 24: 03$ & 108.7362 & 8.695 & 29.846 & 1.52 \\
\hline $07 / 25 / 00$ & $11: 24: 18$ & 108.9862 & 8.688 & 29.844 & 1. \\
\hline $07 / 25 / 00$ & $11: 24: 33$ & 109.2362 & 8.693 & 29.846 & 1.5 \\
\hline $07 / 25 / 00$ & $11: 24: 48$ & 109.4862 & 8.64 & 29.846 & 1.57 \\
\hline $07 / 25 / 00$ & $11: 25: 03$ & 109.7362 & 8.701 & 29.846 & 1.51 \\
\hline $07 / 25 / 00$ & $11: 25: 18$ & 109.9862 & 8.688 & 29.846 & 1. \\
\hline $07 / 25 / 00$ & $11: 25: 33$ & 110.2362 & 8.683 & 29.846 & 1.53 \\
\hline $07 / 25 / 00$ & $11: 25: 48$ & 110.4862 & 8.672 & 29.846 & 1.54 \\
\hline $07 / 25 / 00$ & $11: 26: 03$ & 110.7362 & 8.68 & 29.846 & 1.5 \\
\hline $07 / 25 / 00$ & $11: 26: 18$ & 110.9862 & 8.682 & 29.848 & 1.53 \\
\hline $07 / 25 / 00$ & $11: 26: 33$ & 111.2362 & 8.68 & 29.846 & 1.5 \\
\hline $07 / 25 / 00$ & $11: 26: 48$ & 111.4862 & 8.679 & 29.846 & 1.53 \\
\hline $07 / 25 / 00$ & $11: 27: 03$ & 111.7362 & 8.676 & 29.846 & 1.5 \\
\hline 07/25/00 & $11: 27: 18$ & 111.9862 & 8.794 & 29.846 & 1.4 \\
\hline $07 / 25 / 00$ & $11: 27: 33$ & 112.2362 & 8.752 & 29.846 & 1.46 \\
\hline $07 / 25 / 00$ & $11: 27: 48$ & 112.4862 & 8.706 & 29.846 & 1.51 \\
\hline 07/25/00 & $11: 28: 03$ & 112.7362 & 8.702 & 29.846 & 1.5 \\
\hline $07 / 25 / 00$ & $11: 28: 18$ & 112.9862 & 8.69 & 29.848 & 1.5 \\
\hline $07 / 25 / 00$ & $11: 28: 33$ & 113.2362 & 8.695 & 29.848 & 1.5 \\
\hline $07 / 25 / 00$ & $11: 28: 48$ & 113.4862 & 8.693 & 29.848 & 1.5 \\
\hline $07 / 25 / 00$ & $11: 29: 03$ & 113.7362 & 8.686 & 29.85 & 1.53 \\
\hline $07 / 25 / 00$ & $11: 29: 18$ & 113.9862 & 8.688 & 29.848 & 1. \\
\hline $07 / 25 / 00$ & $11: 29: 33$ & 114.2362 & 8.643 & 29.848 & 1.57 \\
\hline $07 / 25 / 00$ & $11: 29: 48$ & 114.4862 & 8.728 & 29.848 & 1. \\
\hline 07/25/00 & $11: 30: 03$ & 114.7362 & 8.701 & 29.848 & 1.5 \\
\hline $07 / 25 / 00$ & $11: 30: 18$ & 114.9862 & 8.698 & 29.85 & 1. \\
\hline $07 / 25 / 00$ & $11: 30: 33$ & 115.2362 & 8.695 & 29.848 & 1.5 \\
\hline 07/25/00 & $11: 30: 48$ & 115.4862 & 8.683 & 29.85 & 1.5 \\
\hline $07 / 25 / 00$ & $11: 31: 03$ & 115.7362 & 8.688 & 29.85 & 1. \\
\hline $07 / 25 / 00$ & $11: 31: 18$ & 115.9862 & 8.679 & 29.85 & 1.5 \\
\hline $07 / 25 / 00$ & $11: 31: 33$ & 116.2362 & 8.688 & 29.848 & 1. \\
\hline $07 / 25 / 00$ & $11: 31: 48$ & 116.4862 & 8.663 & 29.848 & 1.5 \\
\hline
\end{tabular}




\section{Appendix A (continued)}

\begin{tabular}{|c|c|c|c|c|c|}
\hline 07/25/00 & 11:32:03 & 116.7362 & 8.713 & 29.85 & 1.505 \\
\hline 07/25/00 & 11:32:18 & 116.9862 & 8.695 & 29.848 & 1.523 \\
\hline 07/25/00 & 11:32:33 & 117.2362 & 8.683 & 29.85 & 1.535 \\
\hline 07/25/00 & 11:32:48 & 117.4862 & 8.688 & 29.85 & 1.53 \\
\hline 07/25/00 & 11:33:03 & 117.7362 & 8.686 & 29.85 & 1.532 \\
\hline 07/25/00 & 11:33:18 & 117.9862 & 8.679 & 29.85 & 1.539 \\
\hline 07/25/00 & 11:33:33 & 118.2362 & 8.685 & 29.848 & 1.533 \\
\hline 07/25/00 & 11:33:48 & 118.4862 & 8.688 & 29.848 & 1.53 \\
\hline 07/25/00 & $11: 34: 03$ & 118.7362 & 8.695 & 29.848 & 1.523 \\
\hline 07/25/00 & $11: 34: 18$ & 118.9862 & 8.68 & 29.848 & 1.538 \\
\hline 07/25/00 & 11:34:33 & 119.2362 & 8.696 & 29.848 & 1.522 \\
\hline 07/25/00 & 11:34:48 & 119.4862 & 8.702 & 29.848 & 1.516 \\
\hline 07/25/00 & $11: 35: 03$ & 119.7362 & 8.688 & 29.848 & 1.53 \\
\hline 07/25/00 & 11:35:18 & 119.9862 & 8.692 & 29.848 & 1.526 \\
\hline 07/25/00 & 11:35:33 & 120.2362 & 8.69 & 29.848 & 1.528 \\
\hline 07/25/00 & 11:35:48 & 120.4862 & 8.683 & 29.846 & 1.535 \\
\hline 07/25/00 & 11:36:03 & 120.7362 & 8.692 & 29.848 & 1.526 \\
\hline 07/25/00 & 11:36:18 & 120.9862 & 8.685 & 29.846 & 1.533 \\
\hline 07/25/00 & 11:36:33 & 121.2362 & 8.682 & 29.846 & 1.536 \\
\hline 07/25/00 & $11: 36: 48$ & 121.4862 & 8.69 & 29.848 & 1.528 \\
\hline 07/25/00 & $11: 37: 03$ & 121.7362 & 8.69 & 29.846 & 1.528 \\
\hline 07/25/00 & $11: 37: 18$ & 121.9862 & 8.634 & 29.846 & 1.584 \\
\hline 07/25/00 & 11:37:33 & 122.2362 & 8.708 & 29.846 & 1.51 \\
\hline 07/25/00 & 11:37:48 & 122.4862 & 8.692 & 29.846 & 1.526 \\
\hline 07/25/00 & 11:38:03 & 122.7362 & 8.685 & 29.846 & 1.533 \\
\hline 07/25/00 & 11:38:18 & 122.9862 & 8.683 & 29.846 & 1.535 \\
\hline 07/25/00 & 11:38:33 & 123.2362 & 8.69 & 29.846 & 1.528 \\
\hline 07/25/00 & 11:38:48 & 123.4862 & 8.688 & 29.846 & 1.53 \\
\hline 07/25/00 & $11: 39: 03$ & 123.7362 & 8.695 & 29.846 & 1.523 \\
\hline 07/25/00 & 11:39:18 & 123.9862 & 8.677 & 29.846 & 1.541 \\
\hline 07/25/00 & 11:39:33 & 124.2362 & 8.637 & 29.846 & 1.581 \\
\hline 07/25/00 & $11: 39: 48$ & 124.4862 & 8.726 & 29.846 & 1.492 \\
\hline 07/25/00 & $11: 40: 03$ & 124.7362 & 8.703 & 29.844 & 1.515 \\
\hline 07/25/00 & $11: 40: 18$ & 124.9862 & 8.698 & 29.844 & 1.52 \\
\hline 07/25/00 & $11: 40: 33$ & 125.2362 & 8.679 & 29.846 & 1.539 \\
\hline 07/25/00 & 11:40:48 & 125.4862 & 8.686 & 29.844 & 1.532 \\
\hline 07/25/00 & 11:41:03 & 125.7362 & 8.695 & 29.846 & 1.523 \\
\hline 07/25/00 & 11:41:18 & 125.9862 & 8.679 & 29.844 & 1.539 \\
\hline 07/25/00 & $11: 41: 33$ & 126.2362 & 8.689 & 29.844 & 1.529 \\
\hline 07/25/00 & $11: 41: 48$ & 126.4862 & 8.639 & 29.844 & 1.579 \\
\hline 07/25/00 & 11:42:03 & 126.7362 & 8.713 & 29.844 & 1.505 \\
\hline 07/25/00 & 11:42:18 & 126.9862 & 8.689 & 29.844 & 1.529 \\
\hline 07/25/00 & 11:42:33 & 127.2362 & 8.677 & 29.844 & 1.541 \\
\hline 07/25/00 & 11:42:48 & 127.4862 & 8.666 & 29.846 & 1.552 \\
\hline 07/25/00 & 11:43:03 & 127.7362 & 8.665 & 29.846 & 1.553 \\
\hline 07/25/00 & $11: 43: 18$ & 127.9862 & 8.667 & 29.846 & 1.551 \\
\hline 07/25/00 & 11:43:33 & 128.2362 & 8.67 & 29.846 & 1.548 \\
\hline 07/25/00 & 11:43:48 & 128.4862 & 8.666 & 29.846 & 1.552 \\
\hline 07/25/00 & 11:44:03 & 128.7362 & 8.675 & 29.846 & 1.543 \\
\hline 07/25/00 & 11:44:18 & 128.9862 & 8.679 & 29.844 & 1.539 \\
\hline 07/25/00 & $11: 44: 33$ & 129.2362 & 8.675 & 29.848 & 1.543 \\
\hline
\end{tabular}




\section{Appendix A (continued)}

\begin{tabular}{|c|c|c|c|c|c|}
\hline 07/25/00 & $11: 44: 48$ & 129.4862 & 8.665 & 29.846 & 1.553 \\
\hline $07 / 25 / 00$ & $11: 45: 03$ & 129.7362 & 8.665 & 29.846 & 1.5 \\
\hline 07/25/00 & $11: 45: 18$ & 129.9862 & 8.656 & 29.846 & .5 \\
\hline 07/25/00 & $11: 45: 33$ & 130.2362 & 8.676 & 29.846 & .5 \\
\hline 07/25/00 & $11: 45: 48$ & 130.4862 & 8.665 & 29.846 & .5 \\
\hline 07/25/00 & $11: 46: 03$ & 130.7362 & 8.66 & 29.846 & .5 \\
\hline 07/25/00 & $11: 46: 18$ & 130.9862 & 8.696 & 29.846 & 1.5 \\
\hline 07/25/00 & $11: 46: 33$ & 131.2362 & 8.679 & 29.846 & 1.5 \\
\hline 07/25/00 & $11: 46: 48$ & 131.4862 & 8.666 & 29.846 & 1.55 \\
\hline 07/25/00 & $11: 47: 03$ & 131.7362 & 8.663 & 29.846 & 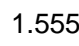 \\
\hline 07/25/00 & $11: 47: 18$ & 131.9862 & 8.662 & 29.846 & 1.5 \\
\hline 07/25/00 & $11: 47: 33$ & 132.2362 & 8.654 & 29.846 & 1.5 \\
\hline $07 / 25 / 00$ & $11: 47: 48$ & 132.4862 & 8.662 & 29.844 & 1.5 \\
\hline 07/25/00 & $11: 48: 03$ & 132.7362 & 8.663 & 29.846 & .5 \\
\hline 07/25/00 & $11: 48: 18$ & 132.9862 & 8.654 & 29.846 & 1.0 \\
\hline $07 / 25 / 00$ & $11: 48: 33$ & 133.2362 & 8.667 & 29.862 & 1.5 \\
\hline 07/25/00 & $11: 48: 48$ & 133.4862 & 8.663 & 29.852 & 1.55 \\
\hline 07/25/00 & $11: 49: 03$ & 133.7362 & 8.66 & 29.848 & 1.55 \\
\hline 07/25/00 & $11: 49: 18$ & 133.9862 & 8.686 & 29.846 & 1.5 \\
\hline 07/25/00 & $11: 49: 33$ & 134.2362 & 8.721 & 29.846 & $1.4 \mathrm{~S}$ \\
\hline 07/25/00 & $11: 49: 48$ & 134.4862 & 8.676 & 29.846 & 1.5 \\
\hline 07/25/00 & $11: 50: 03$ & 134.7362 & 8.667 & 29.844 & 1.5 \\
\hline 07/25/00 & $11: 50: 18$ & 134.9862 & 8.676 & 29.844 & 1.5 \\
\hline $07 / 25 / 00$ & $11: 50: 33$ & 135.2362 & 8.653 & 29.844 & 1.5 \\
\hline 07/25/00 & $11: 50: 48$ & 135.4862 & 8.667 & 29.844 & 1.5 \\
\hline 07/25/00 & $11: 51: 03$ & 135.7362 & 8.659 & 29.844 & 1.5 \\
\hline $07 / 25 / 00$ & $11: 51: 18$ & 135.9862 & 8.653 & 29.846 & 1.56 \\
\hline 07/25/00 & $11: 51: 33$ & 136.2362 & 8.682 & 29.846 & 1.53 \\
\hline 07/25/00 & $11: 51: 48$ & 136.4862 & 8.667 & 29.844 & 1.55 \\
\hline 07/25/00 & $11: 52: 03$ & 136.7362 & 8.663 & 29.846 & 1.5 \\
\hline 07/25/00 & $11: 52: 18$ & 136.9862 & 8.665 & 29.846 & 1.5 \\
\hline 07/25/00 & $11: 52: 33$ & 137.2362 & 8.669 & 29.846 & 1.5 \\
\hline 07/25/00 & $11: 52: 48$ & 137.4862 & 8.666 & 29.844 & 1.5 \\
\hline 07/25/00 & $11: 53: 03$ & 137.7362 & 8.676 & 29.846 & 1.5 \\
\hline 07/25/00 & $11: 53: 18$ & 137.9862 & 8.665 & 29.846 & 1.553 \\
\hline 07/25/00 & 11:53:33 & 138.2362 & 8.662 & 29.846 & 1.5 \\
\hline 07/25/00 & $11: 53: 48$ & 138.4862 & 8.653 & 29.846 & 1.5 \\
\hline 07/25/00 & $11: 54: 03$ & 138.7362 & 8.991 & 29.846 & 1.2 \\
\hline 07/25/00 & $11: 54: 18$ & 138.9862 & 10.133 & 29.846 & 0.0 \\
\hline 07/25/00 & $11: 54: 33$ & 139.2362 & 10.267 & 29.846 & -0.04 \\
\hline 07/25/00 & $11: 54: 48$ & 139.4862 & 10.086 & 29.848 & 0.132 \\
\hline 07/25/00 & $11: 55: 03$ & 139.7362 & 10.041 & 29.862 & 0.17 \\
\hline $07 / 25 / 00$ & $11: 55: 18$ & 139.9862 & 10.033 & 29.852 & 0.1 \\
\hline 07/25/00 & $11: 55: 33$ & 140.2362 & 10.037 & 29.85 & 0.18 \\
\hline 07/25/00 & $11: 55: 48$ & 140.4862 & 10.046 & 29.85 & 0.1 \\
\hline $07 / 25 / 00$ & $11: 56: 03$ & 140.7362 & 10.054 & 29.848 & 0.1 \\
\hline 07/25/00 & $11: 56: 18$ & 140.9862 & 10.06 & 29.848 & 0.1 \\
\hline 07/25/00 & $11: 56: 33$ & 141.2362 & 10.066 & 29.87 & 0.1 \\
\hline 07/25/00 & $11: 56: 48$ & 141.4862 & 10.073 & 29.852 & 0.14 \\
\hline 07/25/00 & $11: 57: 03$ & 141.7362 & 10.079 & 29.85 & 0.13 \\
\hline 07/25/00 & $11: 57: 18$ & 141.9862 & 10.086 & 29.85 & 0.1 \\
\hline
\end{tabular}




\begin{tabular}{lrrrrr}
\multicolumn{7}{c}{ Appendix A (continued) } \\
$07 / 25 / 00$ & $11: 57: 33$ & 142.2362 & 10.09 & 29.848 & 0.128 \\
$07 / 25 / 00$ & $11: 57: 48$ & 142.4862 & 10.093 & 29.872 & 0.125 \\
$07 / 25 / 00$ & $11: 58: 03$ & 142.7362 & 10.097 & 29.854 & 0.121 \\
$07 / 25 / 00$ & $11: 58: 18$ & 142.9862 & 10.102 & 29.85 & 0.116 \\
$07 / 25 / 00$ & $11: 58: 33$ & 143.2362 & 10.105 & 29.85 & 0.113 \\
$07 / 25 / 00$ & $11: 58: 48$ & 143.4862 & 10.107 & 29.85 & 0.111
\end{tabular}




\section{Appendix B - Time-drawdown data for SWP-200C}

SPW 200 C Dynamic 7/27

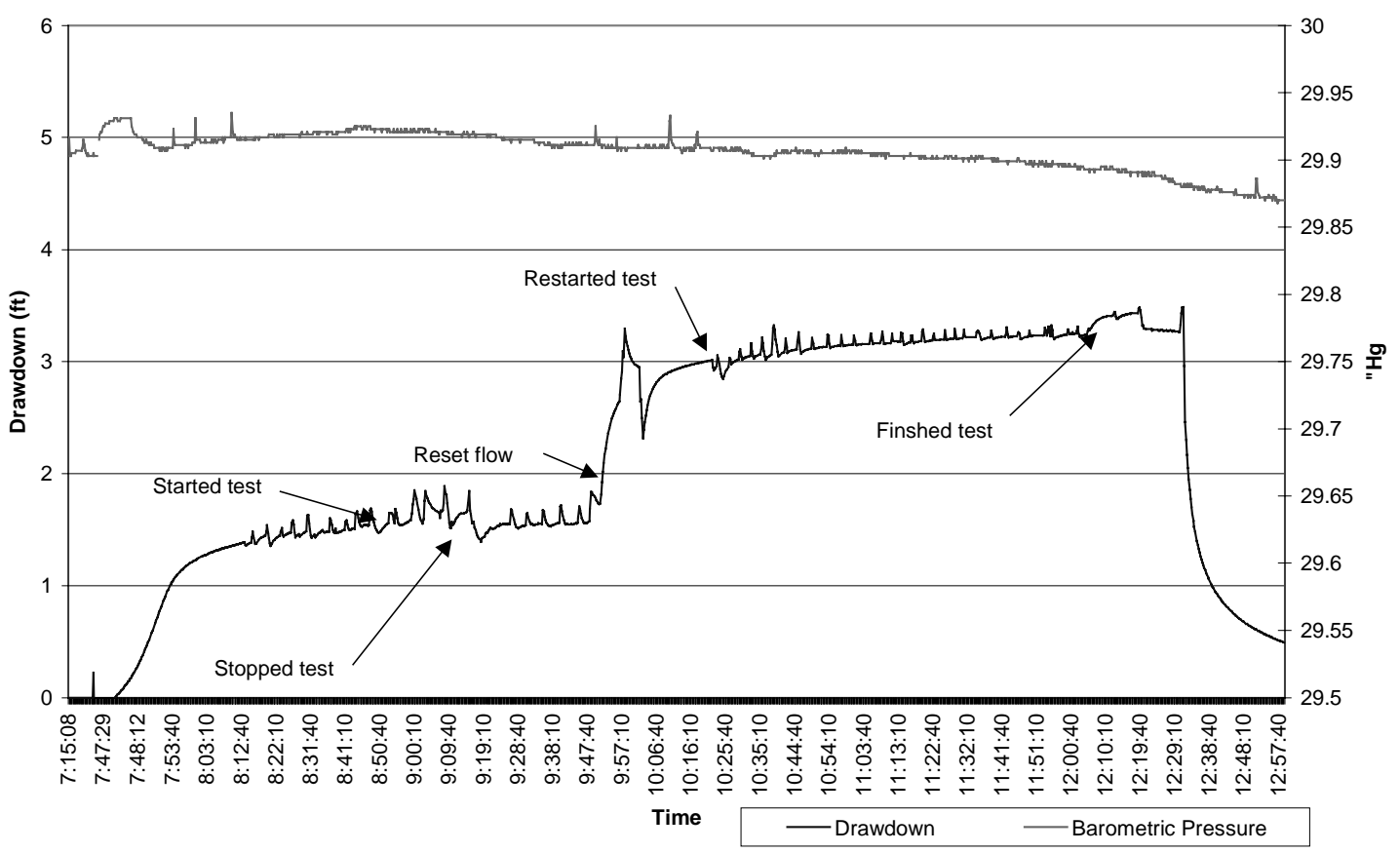

SPW 200 C 7/27

\begin{tabular}{|c|c|c|c|c|c|c|}
\hline & Date & Time & ET (min) & Feet H2O & Inches Hg & $\begin{array}{l}\text { Drawdown } \\
\mathrm{ft}\end{array}$ \\
\hline \multirow[t]{20}{*}{ Background } & $07 / 27 / 00$ & 7:15:08 & 0 & 12.196 & 29.917 & 0 \\
\hline & $07 / 27 / 00$ & 7:16:08 & 1 & 12.199 & 29.903 & -0.003 \\
\hline & $07 / 27 / 00$ & 7:17:08 & 2 & 12.199 & 29.903 & -0.003 \\
\hline & $07 / 27 / 00$ & 7:18:08 & 3 & 12.199 & 29.905 & -0.003 \\
\hline & $07 / 27 / 00$ & 7:19:08 & 4 & 12.199 & 29.905 & -0.003 \\
\hline & $07 / 27 / 00$ & 7:20:08 & 5 & 12.199 & 29.905 & -0.003 \\
\hline & $07 / 27 / 00$ & 7:21:08 & 6 & 12.199 & 29.905 & -0.003 \\
\hline & $07 / 27 / 00$ & 7:22:08 & 7 & 12.199 & 29.905 & -0.003 \\
\hline & $07 / 27 / 00$ & 7:23:08 & 8 & 12.199 & 29.907 & -0.003 \\
\hline & $07 / 27 / 00$ & 7:24:08 & 9 & 12.199 & 29.907 & -0.003 \\
\hline & $07 / 27 / 00$ & 7:25:08 & 10 & 12.198 & 29.907 & -0.002 \\
\hline & $07 / 27 / 00$ & 7:26:08 & 11 & 12.198 & 29.907 & -0.002 \\
\hline & $07 / 27 / 00$ & 7:27:08 & 12 & 12.196 & 29.907 & 0 \\
\hline & $07 / 27 / 00$ & 7:28:08 & 13 & 12.196 & 29.907 & 0 \\
\hline & $07 / 27 / 00$ & 7:29:08 & 14 & 12.196 & 29.907 & 0 \\
\hline & $07 / 27 / 00$ & 7:30:08 & 15 & 12.196 & 29.909 & 0 \\
\hline & $07 / 27 / 00$ & 7:31:08 & 16 & 12.195 & 29.915 & 0.001 \\
\hline & $07 / 27 / 00$ & 7:32:08 & 17 & 12.195 & 29.911 & 0.001 \\
\hline & $07 / 27 / 00$ & 7:33:08 & 18 & 12.195 & 29.907 & 0.001 \\
\hline & $07 / 27 / 00$ & 7:34:08 & 19 & 12.196 & 29.905 & 0 \\
\hline
\end{tabular}




\begin{tabular}{|c|c|c|c|c|c|c|}
\hline & & $\mathbf{A}_{\mathbf{I}}$ & $\operatorname{adix} B$ & atinue & & \\
\hline & $07 / 27 / 00$ & $7: 35: 08$ & 20 & 12.195 & 29.903 & 0.001 \\
\hline & $07 / 27 / 00$ & $7: 36: 08$ & 21 & 12.195 & 29.905 & 0.001 \\
\hline & $07 / 27 / 00$ & $7: 37: 08$ & 22 & 12.195 & 29.903 & 0.001 \\
\hline & $07 / 27 / 00$ & 7:38:08 & 23 & 12.196 & 29.903 & 0 \\
\hline & $07 / 27 / 00$ & 7:39:08 & 24 & 12.196 & 29.903 & 0 \\
\hline & $07 / 27 / 00$ & $7: 40: 08$ & 25 & 12.195 & 29.903 & 0.001 \\
\hline & $07 / 27 / 00$ & $7: 41: 08$ & 26 & 12.196 & 29.903 & 0 \\
\hline & $07 / 27 / 00$ & $7: 42: 08$ & 27 & 11.973 & 29.905 & 0.223 \\
\hline & $07 / 27 / 00$ & $7: 43: 08$ & 28 & 12.391 & 29.903 & -0.195 \\
\hline & $07 / 27 / 00$ & $7: 44: 08$ & 29 & 12.623 & 29.903 & -0.427 \\
\hline & $07 / 27 / 00$ & $7: 45: 08$ & 30 & 12.427 & 29.903 & -0.231 \\
\hline & $07 / 27 / 00$ & $7: 46: 08$ & 31 & 12.453 & 29.903 & -0.257 \\
\hline Pumping & $07 / 27 / 00$ & $7: 47: 26$ & 0 & 12.395 & 29.915 & -0.199 \\
\hline & $07 / 27 / 00$ & $7: 47: 26$ & 0.0112 & 12.395 & 29.919 & -0.199 \\
\hline & $07 / 27 / 00$ & $7: 47: 27$ & 0.0223 & 12.394 & 29.921 & -0.198 \\
\hline & $07 / 27 / 00$ & $7: 47: 28$ & 0.0335 & 12.394 & 29.921 & -0.198 \\
\hline & $07 / 27 / 00$ & $7: 47: 28$ & 0.0447 & 12.386 & 29.923 & -0.19 \\
\hline & $07 / 27 / 00$ & $7: 47: 29$ & 0.0558 & 12.382 & 29.925 & -0.186 \\
\hline & $07 / 27 / 00$ & $7: 47: 30$ & 0.067 & 12.371 & 29.925 & -0.175 \\
\hline & $07 / 27 / 00$ & $7: 47: 30$ & 0.0782 & 12.35 & 29.927 & -0.154 \\
\hline & $07 / 27 / 00$ & $7: 47: 31$ & 0.0893 & 12.329 & 29.927 & -0.133 \\
\hline & $07 / 27 / 00$ & $7: 47: 32$ & 0.1005 & 12.304 & 29.927 & -0.108 \\
\hline & $07 / 27 / 00$ & $7: 47: 32$ & 0.1117 & 12.287 & 29.927 & -0.091 \\
\hline & $07 / 27 / 00$ & $7: 47: 33$ & 0.1228 & 12.267 & 29.927 & -0.071 \\
\hline & $07 / 27 / 00$ & $7: 47: 34$ & 0.134 & 12.254 & 29.929 & -0.058 \\
\hline & $07 / 27 / 00$ & $7: 47: 34$ & 0.1452 & 12.244 & 29.929 & -0.048 \\
\hline & $07 / 27 / 00$ & $7: 47: 35$ & 0.1563 & 12.235 & 29.929 & -0.039 \\
\hline & $07 / 27 / 00$ & $7: 47: 36$ & 0.1675 & 12.219 & 29.929 & -0.023 \\
\hline & $07 / 27 / 00$ & $7: 47: 36$ & 0.1787 & 12.209 & 29.929 & -0.013 \\
\hline & $07 / 27 / 00$ & $7: 47: 37$ & 0.1898 & 12.201 & 29.931 & -0.005 \\
\hline & $07 / 27 / 00$ & $7: 47: 38$ & 0.201 & 12.189 & 29.931 & 0.007 \\
\hline & $07 / 27 / 00$ & $7: 47: 38$ & 0.2122 & 12.182 & 29.931 & 0.014 \\
\hline & $07 / 27 / 00$ & $7: 47: 39$ & 0.2233 & 12.173 & 29.931 & 0.023 \\
\hline & $07 / 27 / 00$ & $7: 47: 40$ & 0.235 & 12.166 & 29.929 & 0.03 \\
\hline & $07 / 27 / 00$ & $7: 47: 40$ & 0.2475 & 12.159 & 29.929 & 0.037 \\
\hline & $07 / 27 / 00$ & $7: 47: 41$ & 0.2607 & 12.147 & 29.929 & 0.049 \\
\hline & $07 / 27 / 00$ & $7: 47: 42$ & 0.2747 & 12.14 & 29.931 & 0.056 \\
\hline & $07 / 27 / 00$ & $7: 47: 43$ & 0.2895 & 12.129 & 29.931 & 0.067 \\
\hline & $07 / 27 / 00$ & $7: 47: 44$ & 0.3052 & 12.121 & 29.931 & 0.075 \\
\hline & $07 / 27 / 00$ & $7: 47: 45$ & 0.3218 & 12.108 & 29.931 & 0.088 \\
\hline & $07 / 27 / 00$ & $7: 47: 46$ & 0.3395 & 12.098 & 29.931 & 0.098 \\
\hline & $07 / 27 / 00$ & $7: 47: 47$ & 0.3582 & 12.087 & 29.931 & 0.109 \\
\hline & $07 / 27 / 00$ & $7: 47: 48$ & 0.378 & 12.078 & 29.931 & 0.118 \\
\hline & $07 / 27 / 00$ & $7: 47: 49$ & 0.399 & 12.065 & 29.931 & 0.131 \\
\hline & $07 / 27 / 00$ & $7: 47: 51$ & 0.4212 & 12.055 & 29.931 & 0.141 \\
\hline & $07 / 27 / 00$ & $7: 47: 52$ & 0.4447 & 12.044 & 29.931 & 0.152 \\
\hline & $07 / 27 / 00$ & $7: 47: 54$ & 0.4695 & 12.032 & 29.931 & 0.164 \\
\hline & $07 / 27 / 00$ & $7: 47: 55$ & 0.4958 & 12.023 & 29.931 & 0.173 \\
\hline & $07 / 27 / 00$ & $7: 47: 57$ & 0.5238 & 12.006 & 29.925 & 0.19 \\
\hline & $07 / 27 / 00$ & 7:47:59 & 0.5535 & 11.995 & 29.923 & 0.201 \\
\hline
\end{tabular}




\begin{tabular}{|c|c|c|c|c|c|}
\hline \multicolumn{6}{|c|}{ Appendix B (continued) } \\
\hline 07/27/00 & 7:48:01 & 0.5848 & 11.979 & 29.921 & 0.217 \\
\hline $07 / 27 / 00$ & $7: 48: 03$ & 0.618 & 11.966 & 29.919 & 0.23 \\
\hline 07/27/00 & $7: 48: 05$ & 0.6532 & 11.95 & 29.919 & 0.246 \\
\hline 07/27/00 & $7: 48: 07$ & 0.6905 & 11.937 & 29.919 & 0.259 \\
\hline 07/27/00 & 7:48:09 & 0.73 & 11.921 & 29.917 & 0.275 \\
\hline 07/27/00 & $7: 48: 12$ & 0.7718 & 11.905 & 29.917 & 0.291 \\
\hline 07/27/00 & $7: 48: 14$ & 0.8162 & 11.889 & 29.917 & 0.307 \\
\hline 07/27/00 & $7: 48: 17$ & 0.8632 & 11.872 & 29.917 & 0.324 \\
\hline 07/27/00 & $7: 48: 20$ & 0.913 & 11.855 & 29.917 & 0.341 \\
\hline 07/27/00 & $7: 48: 23$ & 0.9657 & 11.838 & 29.917 & 0.358 \\
\hline 07/27/00 & $7: 48: 27$ & 1.0215 & 11.819 & 29.915 & 0.377 \\
\hline 07/27/00 & $7: 48: 30$ & 1.0807 & 11.801 & 29.915 & 0.395 \\
\hline $07 / 27 / 00$ & $7: 48: 34$ & 1.1433 & 11.784 & 29.913 & 0.412 \\
\hline 07/27/00 & $7: 48: 38$ & 1.2097 & 11.763 & 29.913 & 0.433 \\
\hline 07/27/00 & $7: 48: 42$ & 1.28 & 11.742 & 29.915 & 0.454 \\
\hline $07 / 27 / 00$ & $7: 48: 47$ & 1.3545 & 11.724 & 29.913 & 0.472 \\
\hline 07/27/00 & $7: 48: 52$ & 1.4335 & 11.702 & 29.913 & 0.494 \\
\hline $07 / 27 / 00$ & $7: 48: 57$ & 1.5172 & 11.682 & 29.911 & 0.514 \\
\hline 07/27/00 & 7:49:02 & 1.6057 & 11.66 & 29.913 & 0.536 \\
\hline $07 / 27 / 00$ & 7:49:07 & 1.6995 & 11.639 & 29.913 & 0.557 \\
\hline 07/27/00 & $7: 49: 13$ & 1.7988 & 11.619 & 29.911 & 0.577 \\
\hline $07 / 27 / 00$ & $7: 49: 20$ & 1.9042 & 11.595 & 29.911 & 0.601 \\
\hline $07 / 27 / 00$ & 7:49:26 & 2.0157 & 11.572 & 29.911 & 0.624 \\
\hline $07 / 27 / 00$ & $7: 49: 34$ & 2.1338 & 11.551 & 29.909 & 0.645 \\
\hline $07 / 27 / 00$ & $7: 49: 41$ & 2.259 & 11.528 & 29.909 & 0.668 \\
\hline 07/27/00 & $7: 49: 49$ & 2.3915 & 11.506 & 29.909 & 0.69 \\
\hline 07/27/00 & $7: 49: 57$ & 2.532 & 11.483 & 29.909 & 0.713 \\
\hline 07/27/00 & 7:50:06 & 2.6808 & 11.461 & 29.909 & 0.735 \\
\hline $07 / 27 / 00$ & $7: 50: 16$ & 2.8383 & 11.438 & 29.909 & 0.758 \\
\hline $07 / 27 / 00$ & $7: 50: 26$ & 3.0052 & 11.417 & 29.907 & 0.779 \\
\hline $07 / 27 / 00$ & $7: 50: 36$ & 3.182 & 11.394 & 29.909 & 0.802 \\
\hline $07 / 27 / 00$ & $7: 50: 48$ & 3.3693 & 11.374 & 29.907 & 0.822 \\
\hline $07 / 27 / 00$ & $7: 51: 00$ & 3.5677 & 11.352 & 29.909 & 0.844 \\
\hline 07/27/00 & $7: 51: 12$ & 3.7778 & 11.33 & 29.909 & 0.866 \\
\hline $07 / 27 / 00$ & $7: 51: 26$ & 4.0005 & 11.31 & 29.907 & 0.886 \\
\hline $07 / 27 / 00$ & $7: 51: 40$ & 4.2363 & 11.29 & 29.907 & 0.906 \\
\hline 07/27/00 & $7: 51: 55$ & 4.4862 & 11.268 & 29.909 & 0.928 \\
\hline $07 / 27 / 00$ & $7: 52: 10$ & 4.7362 & 11.248 & 29.907 & 0.948 \\
\hline $07 / 27 / 00$ & $7: 52: 25$ & 4.9862 & 11.231 & 29.909 & 0.965 \\
\hline 07/27/00 & $7: 52: 40$ & 5.2362 & 11.214 & 29.909 & 0.982 \\
\hline $07 / 27 / 00$ & $7: 52: 55$ & 5.4862 & 11.196 & 29.909 & 1 \\
\hline $07 / 27 / 00$ & $7: 53: 10$ & 5.7362 & 11.183 & 29.909 & 1.013 \\
\hline 07/27/00 & $7: 53: 25$ & 5.9862 & 11.17 & 29.909 & 1.026 \\
\hline 07/27/00 & $7: 53: 40$ & 6.2362 & 11.155 & 29.909 & 1.041 \\
\hline 07/27/00 & $7: 53: 55$ & 6.4862 & 11.142 & 29.923 & 1.054 \\
\hline $07 / 27 / 00$ & $7: 54: 10$ & 6.7362 & 11.133 & 29.913 & 1.063 \\
\hline $07 / 27 / 00$ & $7: 54: 25$ & 6.9862 & 11.122 & 29.911 & 1.074 \\
\hline $07 / 27 / 00$ & $7: 54: 40$ & 7.2362 & 11.109 & 29.911 & 1.087 \\
\hline $07 / 27 / 00$ & $7: 54: 55$ & 7.4862 & 11.1 & 29.911 & 1.096 \\
\hline $07 / 27 / 00$ & $7: 55: 10$ & 7.7362 & 11.091 & 29.911 & 1.105 \\
\hline $07 / 27 / 00$ & $7: 55: 25$ & 7.9862 & 11.083 & 29.911 & 1.113 \\
\hline
\end{tabular}




\section{Appendix B (continued)}

\begin{tabular}{|c|c|c|c|c|c|}
\hline $07 / 27 / 00$ & $7: 55: 40$ & 8.2362 & 11.073 & 29.911 & 1.123 \\
\hline $07 / 27 / 00$ & $7: 55: 55$ & 8.4862 & 11.064 & 29.911 & 1.132 \\
\hline $07 / 27 / 00$ & $7: 56: 10$ & 8.7362 & 11.057 & 29.911 & 1.139 \\
\hline $07 / 27 / 00$ & $7: 56: 25$ & 8.9862 & 11.049 & 29.911 & 1.147 \\
\hline $07 / 27 / 00$ & $7: 56: 40$ & 9.2362 & 11.044 & 29.911 & 1.152 \\
\hline $07 / 27 / 00$ & 7:56:55 & 9.4862 & 11.034 & 29.911 & 1.162 \\
\hline $07 / 27 / 00$ & $7: 57: 10$ & 9.7362 & 11.028 & 29.909 & 1.168 \\
\hline $07 / 27 / 00$ & $7: 57: 25$ & 9.9862 & 11.021 & 29.911 & 1.175 \\
\hline $07 / 27 / 00$ & $7: 57: 40$ & 10.2362 & 11.015 & 29.911 & 1.181 \\
\hline $07 / 27 / 00$ & 7:57:55 & 10.4862 & 11.008 & 29.909 & 1.188 \\
\hline $07 / 27 / 00$ & 7:58:10 & 10.7362 & 11.003 & 29.911 & 1.193 \\
\hline $07 / 27 / 00$ & $7: 58: 25$ & 10.9862 & 10.995 & 29.911 & 1.201 \\
\hline $07 / 27 / 00$ & 7:58:40 & 11.2362 & 10.99 & 29.911 & 1.206 \\
\hline $07 / 27 / 00$ & 7:58:55 & 11.4862 & 10.986 & 29.911 & 1.21 \\
\hline $07 / 27 / 00$ & 7:59:10 & 11.7362 & 10.982 & 29.913 & 1.214 \\
\hline $07 / 27 / 00$ & $7: 59: 25$ & 11.9862 & 10.975 & 29.913 & 1.221 \\
\hline $07 / 27 / 00$ & $7: 59: 40$ & 12.2362 & 10.972 & 29.913 & 1.224 \\
\hline $07 / 27 / 00$ & 7:59:55 & 12.4862 & 10.967 & 29.931 & 1.229 \\
\hline $07 / 27 / 00$ & $8: 00: 10$ & 12.7362 & 10.962 & 29.915 & 1.234 \\
\hline $07 / 27 / 00$ & 8:00:25 & 12.9862 & 10.959 & 29.915 & 1.237 \\
\hline $07 / 27 / 00$ & 8:00:40 & 13.2362 & 10.952 & 29.915 & 1.244 \\
\hline $07 / 27 / 00$ & 8:00:55 & 13.4862 & 10.947 & 29.915 & 1.249 \\
\hline $07 / 27 / 00$ & 8:01:10 & 13.7362 & 10.944 & 29.915 & 1.252 \\
\hline $07 / 27 / 00$ & $8: 01: 25$ & 13.9862 & 10.94 & 29.913 & 1.256 \\
\hline $07 / 27 / 00$ & 8:01:40 & 14.2362 & 10.936 & 29.913 & 1.26 \\
\hline $07 / 27 / 00$ & 8:01:55 & 14.4862 & 10.933 & 29.913 & 1.263 \\
\hline $07 / 27 / 00$ & 8:02:10 & 14.7362 & 10.928 & 29.913 & 1.268 \\
\hline $07 / 27 / 00$ & 8:02:25 & 14.9862 & 10.926 & 29.913 & 1.27 \\
\hline $07 / 27 / 00$ & 8:02:40 & 15.2362 & 10.923 & 29.915 & 1.273 \\
\hline $07 / 27 / 00$ & 8:02:55 & 15.4862 & 10.92 & 29.913 & 1.276 \\
\hline $07 / 27 / 00$ & 8:03:10 & 15.7362 & 10.917 & 29.913 & 1.279 \\
\hline $07 / 27 / 00$ & 8:03:25 & 15.9862 & 10.91 & 29.913 & 1.286 \\
\hline $07 / 27 / 00$ & 8:03:40 & 16.2362 & 10.908 & 29.913 & 1.288 \\
\hline $07 / 27 / 00$ & 8:03:55 & 16.4862 & 10.904 & 29.913 & 1.292 \\
\hline $07 / 27 / 00$ & 8:04:10 & 16.7362 & 10.901 & 29.913 & 1.295 \\
\hline $07 / 27 / 00$ & 8:04:25 & 16.9862 & 10.898 & 29.915 & 1.298 \\
\hline $07 / 27 / 00$ & 8:04:40 & 17.2362 & 10.894 & 29.915 & 1.302 \\
\hline $07 / 27 / 00$ & 8:04:55 & 17.4862 & 10.891 & 29.913 & 1.305 \\
\hline $07 / 27 / 00$ & $8: 05: 10$ & 17.7362 & 10.888 & 29.915 & 1.308 \\
\hline $07 / 27 / 00$ & $8: 05: 25$ & 17.9862 & 10.885 & 29.915 & 1.311 \\
\hline $07 / 27 / 00$ & 8:05:40 & 18.2362 & 10.882 & 29.913 & 1.314 \\
\hline $07 / 27 / 00$ & 8:05:55 & 18.4862 & 10.88 & 29.913 & 1.316 \\
\hline $07 / 27 / 00$ & $8: 06: 10$ & 18.7362 & 10.878 & 29.915 & 1.318 \\
\hline $07 / 27 / 00$ & 8:06:25 & 18.9862 & 10.875 & 29.915 & 1.321 \\
\hline $07 / 27 / 00$ & 8:06:40 & 19.2362 & 10.871 & 29.915 & 1.325 \\
\hline $07 / 27 / 00$ & 8:06:55 & 19.4862 & 10.869 & 29.915 & 1.327 \\
\hline $07 / 27 / 00$ & 8:07:10 & 19.7362 & 10.865 & 29.915 & 1.331 \\
\hline $07 / 27 / 00$ & 8:07:25 & 19.9862 & 10.864 & 29.917 & 1.332 \\
\hline $07 / 27 / 00$ & 8:07:40 & 20.2362 & 10.862 & 29.913 & 1.334 \\
\hline $07 / 27 / 00$ & 8:07:55 & 20.4862 & 10.859 & 29.915 & 1.337 \\
\hline $07 / 27 / 00$ & 8:08:10 & 20.7362 & 10.856 & 29.917 & 1.34 \\
\hline
\end{tabular}




\begin{tabular}{|c|c|c|c|c|c|}
\hline \multicolumn{6}{|c|}{ Appendix B (continued) } \\
\hline 07/27/00 & 8:08:25 & 20.9862 & 10.855 & 29.917 & 1.341 \\
\hline $07 / 27 / 00$ & $8: 08: 40$ & 21.2362 & 10.852 & 29.917 & 1.344 \\
\hline 07/27/00 & 8:08:55 & 21.4862 & 10.851 & 29.917 & 1.345 \\
\hline 07/27/00 & 8:09:10 & 21.7362 & 10.848 & 29.917 & 1.348 \\
\hline 07/27/00 & 8:09:25 & 21.9862 & 10.845 & 29.917 & 1.351 \\
\hline 07/27/00 & 8:09:40 & 22.2362 & 10.844 & 29.917 & 1.352 \\
\hline 07/27/00 & $8: 09: 55$ & 22.4862 & 10.842 & 29.935 & 1.354 \\
\hline $07 / 27 / 00$ & $8: 10: 10$ & 22.7362 & 10.838 & 29.921 & 1.358 \\
\hline 07/27/00 & $8: 10: 25$ & 22.9862 & 10.836 & 29.917 & 1.36 \\
\hline 07/27/00 & $8: 10: 40$ & 23.2362 & 10.833 & 29.919 & 1.363 \\
\hline 07/27/00 & $8: 10: 55$ & 23.4862 & 10.832 & 29.917 & 1.364 \\
\hline 07/27/00 & $8: 11: 10$ & 23.7362 & 10.828 & 29.917 & 1.368 \\
\hline $07 / 27 / 00$ & $8: 11: 25$ & 23.9862 & 10.826 & 29.915 & 1.37 \\
\hline 07/27/00 & $8: 11: 40$ & 24.2362 & 10.825 & 29.915 & 1.371 \\
\hline 07/27/00 & $8: 11: 55$ & 24.4862 & 10.823 & 29.915 & 1.373 \\
\hline $07 / 27 / 00$ & $8: 12: 10$ & 24.7362 & 10.82 & 29.917 & 1.376 \\
\hline 07/27/00 & $8: 12: 25$ & 24.9862 & 10.818 & 29.915 & 1.378 \\
\hline $07 / 27 / 00$ & $8: 12: 40$ & 25.2362 & 10.816 & 29.915 & 1.38 \\
\hline $07 / 27 / 00$ & $8: 12: 55$ & 25.4862 & 10.815 & 29.915 & 1.381 \\
\hline $07 / 27 / 00$ & $8: 13: 10$ & 25.7362 & 10.813 & 29.915 & 1.383 \\
\hline $07 / 27 / 00$ & 8:13:25 & 25.9862 & 10.81 & 29.915 & 1.386 \\
\hline $07 / 27 / 00$ & $8: 13: 40$ & 26.2362 & 10.833 & 29.917 & 1.363 \\
\hline 07/27/00 & $8: 13: 55$ & 26.4862 & 10.836 & 29.915 & 1.36 \\
\hline $07 / 27 / 00$ & $8: 14: 10$ & 26.7362 & 10.829 & 29.915 & 1.367 \\
\hline $07 / 27 / 00$ & $8: 14: 25$ & 26.9862 & 10.823 & 29.915 & 1.373 \\
\hline 07/27/00 & $8: 14: 40$ & 27.2362 & 10.82 & 29.915 & 1.376 \\
\hline 07/27/00 & $8: 14: 55$ & 27.4862 & 10.816 & 29.915 & 1.38 \\
\hline 07/27/00 & $8: 15: 10$ & 27.7362 & 10.813 & 29.915 & 1.383 \\
\hline $07 / 27 / 00$ & $8: 15: 25$ & 27.9862 & 10.764 & 29.915 & 1.432 \\
\hline $07 / 27 / 00$ & $8: 15: 40$ & 28.2362 & 10.715 & 29.915 & 1.481 \\
\hline $07 / 27 / 00$ & $8: 15: 55$ & 28.4862 & 10.761 & 29.915 & 1.435 \\
\hline $07 / 27 / 00$ & $8: 16: 10$ & 28.7362 & 10.806 & 29.917 & 1.39 \\
\hline $07 / 27 / 00$ & $8: 16: 25$ & 28.9862 & 10.823 & 29.915 & 1.373 \\
\hline 07/27/00 & $8: 16: 40$ & 29.2362 & 10.822 & 29.917 & 1.374 \\
\hline $07 / 27 / 00$ & $8: 16: 55$ & 29.4862 & 10.807 & 29.917 & 1.389 \\
\hline 07/27/00 & $8: 17: 10$ & 29.7362 & 10.797 & 29.917 & 1.399 \\
\hline 07/27/00 & $8: 17: 25$ & 29.9862 & 10.786 & 29.915 & 1.41 \\
\hline $07 / 27 / 00$ & $8: 17: 40$ & 30.2362 & 10.777 & 29.917 & 1.419 \\
\hline $07 / 27 / 00$ & $8: 17: 55$ & 30.4862 & 10.77 & 29.917 & 1.426 \\
\hline 07/27/00 & $8: 18: 10$ & 30.7362 & 10.767 & 29.917 & 1.429 \\
\hline $07 / 27 / 00$ & 8:18:25 & 30.9862 & 10.761 & 29.917 & 1.435 \\
\hline $07 / 27 / 00$ & 8:18:40 & 31.2362 & 10.757 & 29.917 & 1.439 \\
\hline 07/27/00 & $8: 18: 55$ & 31.4862 & 10.753 & 29.917 & 1.443 \\
\hline 07/27/00 & 8:19:10 & 31.7362 & 10.75 & 29.917 & 1.446 \\
\hline 07/27/00 & 8:19:25 & 31.9862 & 10.712 & 29.917 & 1.484 \\
\hline 07/27/00 & $8: 19: 40$ & 32.2362 & 10.658 & 29.917 & 1.538 \\
\hline $07 / 27 / 00$ & $8: 19: 55$ & 32.4862 & 10.725 & 29.917 & 1.471 \\
\hline $07 / 27 / 00$ & $8: 20: 10$ & 32.7362 & 10.777 & 29.919 & 1.419 \\
\hline $07 / 27 / 00$ & $8: 20: 25$ & 32.9862 & 10.819 & 29.919 & 1.377 \\
\hline $07 / 27 / 00$ & $8: 20: 40$ & 33.2362 & 10.842 & 29.919 & 1.354 \\
\hline $07 / 27 / 00$ & 8:20:55 & 33.4862 & 10.823 & 29.919 & 1.373 \\
\hline
\end{tabular}




\section{Appendix B (continued)}

\begin{tabular}{|c|c|c|c|c|c|}
\hline $07 / 27 / 00$ & $8: 21: 10$ & 33.7362 & 10.809 & 29.919 & 1.387 \\
\hline $07 / 27 / 00$ & $8: 21: 25$ & 33.9862 & 10.796 & 29.919 & 1.4 \\
\hline $07 / 27 / 00$ & $8: 21: 40$ & 34.2362 & 10.786 & 29.917 & 1.41 \\
\hline $07 / 27 / 00$ & 8:21:55 & 34.4862 & 10.777 & 29.917 & 1.419 \\
\hline $07 / 27 / 00$ & 8:22:10 & 34.7362 & 10.77 & 29.917 & 1.426 \\
\hline $07 / 27 / 00$ & 8:22:25 & 34.9862 & 10.763 & 29.919 & 1.433 \\
\hline $07 / 27 / 00$ & 8:22:40 & 35.2362 & 10.757 & 29.917 & 1.439 \\
\hline $07 / 27 / 00$ & 8:22:55 & 35.4862 & 10.753 & 29.917 & 1.443 \\
\hline $07 / 27 / 00$ & $8: 23: 10$ & 35.7362 & 10.75 & 29.919 & 1.446 \\
\hline $07 / 27 / 00$ & 8:23:25 & 35.9862 & 10.712 & 29.919 & 1.484 \\
\hline $07 / 27 / 00$ & 8:23:40 & 36.2362 & 10.681 & 29.919 & 1.515 \\
\hline $07 / 27 / 00$ & 8:23:55 & 36.4862 & 10.738 & 29.917 & 1.458 \\
\hline $07 / 27 / 00$ & $8: 24: 10$ & 36.7362 & 10.756 & 29.917 & 1.44 \\
\hline $07 / 27 / 00$ & $8: 24: 25$ & 36.9862 & 10.75 & 29.919 & 1.446 \\
\hline $07 / 27 / 00$ & $8: 24: 40$ & 37.2362 & 10.743 & 29.919 & 1.453 \\
\hline $07 / 27 / 00$ & $8: 24: 55$ & 37.4862 & 10.738 & 29.919 & 1.458 \\
\hline $07 / 27 / 00$ & $8: 25: 10$ & 37.7362 & 10.734 & 29.919 & 1.462 \\
\hline $07 / 27 / 00$ & $8: 25: 25$ & 37.9862 & 10.731 & 29.919 & 1.465 \\
\hline $07 / 27 / 00$ & $8: 25: 40$ & 38.2362 & 10.727 & 29.919 & 1.469 \\
\hline $07 / 27 / 00$ & $8: 25: 55$ & 38.4862 & 10.724 & 29.919 & 1.472 \\
\hline $07 / 27 / 00$ & $8: 26: 10$ & 38.7362 & 10.723 & 29.919 & 1.473 \\
\hline $07 / 27 / 00$ & $8: 26: 25$ & 38.9862 & 10.633 & 29.919 & 1.563 \\
\hline $07 / 27 / 00$ & 8:26:40 & 39.2362 & 10.612 & 29.919 & 1.584 \\
\hline $07 / 27 / 00$ & 8:26:55 & 39.4862 & 10.65 & 29.919 & 1.546 \\
\hline $07 / 27 / 00$ & $8: 27: 10$ & 39.7362 & 10.718 & 29.919 & 1.478 \\
\hline $07 / 27 / 00$ & $8: 27: 25$ & 39.9862 & 10.764 & 29.919 & 1.432 \\
\hline $07 / 27 / 00$ & $8: 27: 40$ & 40.2362 & 10.747 & 29.919 & 1.449 \\
\hline $07 / 27 / 00$ & $8: 27: 55$ & 40.4862 & 10.751 & 29.919 & 1.445 \\
\hline $07 / 27 / 00$ & $8: 28: 10$ & 40.7362 & 10.743 & 29.919 & 1.453 \\
\hline $07 / 27 / 00$ & 8:28:25 & 40.9862 & 10.737 & 29.919 & 1.459 \\
\hline $07 / 27 / 00$ & $8: 28: 40$ & 41.2362 & 10.756 & 29.917 & 1.44 \\
\hline $07 / 27 / 00$ & 8:28:55 & 41.4862 & 10.746 & 29.919 & 1.45 \\
\hline $07 / 27 / 00$ & $8: 29: 10$ & 41.7362 & 10.741 & 29.921 & 1.455 \\
\hline $07 / 27 / 00$ & $8: 29: 25$ & 41.9862 & 10.734 & 29.919 & 1.462 \\
\hline $07 / 27 / 00$ & 8:29:40 & 42.2362 & 10.728 & 29.919 & 1.468 \\
\hline $07 / 27 / 00$ & 8:29:55 & 42.4862 & 10.721 & 29.919 & 1.475 \\
\hline $07 / 27 / 00$ & $8: 30: 10$ & 42.7362 & 10.715 & 29.919 & 1.481 \\
\hline $07 / 27 / 00$ & $8: 30: 25$ & 42.9862 & 10.711 & 29.919 & 1.485 \\
\hline $07 / 27 / 00$ & 8:30:40 & 43.2362 & 10.571 & 29.921 & 1.625 \\
\hline $07 / 27 / 00$ & $8: 30: 55$ & 43.4862 & 10.566 & 29.919 & 1.63 \\
\hline $07 / 27 / 00$ & $8: 31: 10$ & 43.7362 & 10.638 & 29.919 & 1.558 \\
\hline $07 / 27 / 00$ & $8: 31: 25$ & 43.9862 & 10.701 & 29.919 & 1.495 \\
\hline $07 / 27 / 00$ & $8: 31: 40$ & 44.2362 & 10.753 & 29.919 & 1.443 \\
\hline $07 / 27 / 00$ & $8: 31: 55$ & 44.4862 & 10.769 & 29.919 & 1.427 \\
\hline $07 / 27 / 00$ & 8:32:10 & 44.7362 & 10.757 & 29.919 & 1.439 \\
\hline $07 / 27 / 00$ & $8: 32: 25$ & 44.9862 & 10.746 & 29.921 & 1.45 \\
\hline $07 / 27 / 00$ & 8:32:40 & 45.2362 & 10.74 & 29.921 & 1.456 \\
\hline $07 / 27 / 00$ & 8:32:55 & 45.4862 & 10.766 & 29.921 & 1.43 \\
\hline $07 / 27 / 00$ & 8:33:10 & 45.7362 & 10.753 & 29.921 & 1.443 \\
\hline $07 / 27 / 00$ & 8:33:25 & 45.9862 & 10.743 & 29.919 & 1.453 \\
\hline $07 / 27 / 00$ & 8:33:40 & 46.2362 & 10.734 & 29.921 & 1.462 \\
\hline
\end{tabular}




\begin{tabular}{|c|c|c|c|c|c|}
\hline \multicolumn{6}{|c|}{ Appendix B (continued) } \\
\hline 07/27/00 & 8:33:55 & 46.4862 & 10.724 & 29.919 & 1.472 \\
\hline $07 / 27 / 00$ & $8: 34: 10$ & 46.7362 & 10.72 & 29.921 & 1.476 \\
\hline 07/27/00 & $8: 34: 25$ & 46.9862 & 10.712 & 29.921 & 1.484 \\
\hline $07 / 27 / 00$ & $8: 34: 40$ & 47.2362 & 10.708 & 29.921 & 1.488 \\
\hline 07/27/00 & $8: 34: 55$ & 47.4862 & 10.699 & 29.921 & 1.497 \\
\hline 07/27/00 & 8:35:10 & 47.7362 & 10.717 & 29.921 & 1.479 \\
\hline 07/27/00 & $8: 35: 25$ & 47.9862 & 10.72 & 29.921 & 1.476 \\
\hline 07/27/00 & $8: 35: 40$ & 48.2362 & 10.717 & 29.921 & 1.479 \\
\hline 07/27/00 & 8:35:55 & 48.4862 & 10.717 & 29.921 & 1.479 \\
\hline 07/27/00 & $8: 36: 10$ & 48.7362 & 10.715 & 29.919 & 1.481 \\
\hline $07 / 27 / 00$ & 8:36:25 & 48.9862 & 10.714 & 29.921 & 1.482 \\
\hline 07/27/00 & 8:36:40 & 49.2362 & 10.714 & 29.921 & 1.482 \\
\hline 07/27/00 & 8:36:55 & 49.4862 & 10.597 & 29.921 & 1.599 \\
\hline 07/27/00 & 8:37:10 & 49.7362 & 10.607 & 29.921 & 1.589 \\
\hline 07/27/00 & 8:37:25 & 49.9862 & 10.653 & 29.921 & 1.543 \\
\hline $07 / 27 / 00$ & $8: 37: 40$ & 50.2362 & 10.695 & 29.919 & 1.501 \\
\hline 07/27/00 & 8:37:55 & 50.4862 & 10.717 & 29.919 & 1.479 \\
\hline 07/27/00 & 8:38:10 & 50.7362 & 10.728 & 29.919 & 1.468 \\
\hline 07/27/00 & 8:38:25 & 50.9862 & 10.686 & 29.919 & 1.51 \\
\hline 07/27/00 & 8:38:40 & 51.2362 & 10.725 & 29.921 & 1.471 \\
\hline 07/27/00 & $8: 38: 55$ & 51.4862 & 10.721 & 29.919 & 1.475 \\
\hline $07 / 27 / 00$ & 8:39:10 & 51.7362 & 10.72 & 29.921 & 1.476 \\
\hline 07/27/00 & 8:39:25 & 51.9862 & 10.718 & 29.921 & 1.478 \\
\hline 07/27/00 & 8:39:40 & 52.2362 & 10.715 & 29.921 & 1.481 \\
\hline 07/27/00 & 8:39:55 & 52.4862 & 10.714 & 29.921 & 1.482 \\
\hline 07/27/00 & 8:40:10 & 52.7362 & 10.708 & 29.921 & 1.488 \\
\hline 07/27/00 & $8: 40: 25$ & 52.9862 & 10.705 & 29.921 & 1.491 \\
\hline 07/27/00 & $8: 40: 40$ & 53.2362 & 10.702 & 29.921 & 1.494 \\
\hline 07/27/00 & 8:40:55 & 53.4862 & 10.702 & 29.921 & 1.494 \\
\hline 07/27/00 & $8: 41: 10$ & 53.7362 & 10.629 & 29.921 & 1.567 \\
\hline 07/27/00 & 8:41:25 & 53.9862 & 10.613 & 29.923 & 1.583 \\
\hline 07/27/00 & $8: 41: 40$ & 54.2362 & 10.649 & 29.923 & 1.547 \\
\hline 07/27/00 & $8: 41: 55$ & 54.4862 & 10.678 & 29.921 & 1.518 \\
\hline 07/27/00 & 8:42:10 & 54.7362 & 10.694 & 29.923 & 1.502 \\
\hline 07/27/00 & $8: 42: 25$ & 54.9862 & 10.701 & 29.921 & 1.495 \\
\hline 07/27/00 & $8: 42: 40$ & 55.2362 & 10.695 & 29.923 & 1.501 \\
\hline $07 / 27 / 00$ & 8:42:55 & 55.4862 & 10.688 & 29.923 & 1.508 \\
\hline 07/27/00 & 8:43:10 & 55.7362 & 10.685 & 29.923 & 1.511 \\
\hline 07/27/00 & 8:43:25 & 55.9862 & 10.686 & 29.923 & 1.51 \\
\hline 07/27/00 & 8:43:40 & 56.2362 & 10.689 & 29.925 & 1.507 \\
\hline 07/27/00 & 8:43:55 & 56.4862 & 10.56 & 29.925 & 1.636 \\
\hline 07/27/00 & 8:44:10 & 56.7362 & 10.545 & 29.923 & 1.651 \\
\hline $07 / 27 / 00$ & $8: 44: 25$ & 56.9862 & 10.535 & 29.925 & 1.661 \\
\hline 07/27/00 & $8: 44: 40$ & 57.2362 & 10.591 & 29.925 & 1.605 \\
\hline 07/27/00 & 8:44:55 & 57.4862 & 10.629 & 29.925 & 1.567 \\
\hline 07/27/00 & $8: 45: 10$ & 57.7362 & 10.649 & 29.925 & 1.547 \\
\hline 07/27/00 & 8:45:25 & 57.9862 & 10.663 & 29.925 & 1.533 \\
\hline 07/27/00 & $8: 45: 40$ & 58.2362 & 10.672 & 29.923 & 1.524 \\
\hline $07 / 27 / 00$ & $8: 45: 55$ & 58.4862 & 10.666 & 29.923 & 1.53 \\
\hline 07/27/00 & $8: 46: 10$ & 58.7362 & 10.659 & 29.925 & 1.537 \\
\hline $07 / 27 / 00$ & $8: 46: 25$ & 58.9862 & 10.653 & 29.925 & 1.543 \\
\hline
\end{tabular}




\section{Appendix B (continued)}

\begin{tabular}{|c|c|c|c|c|c|}
\hline $07 / 27 / 00$ & $8: 46: 40$ & 59.2362 & 10.653 & 29.925 & 1.543 \\
\hline $07 / 27 / 00$ & $8: 46: 55$ & 59.4862 & 10.659 & 29.923 & 1.537 \\
\hline $07 / 27 / 00$ & $8: 47: 10$ & 59.7362 & 10.658 & 29.923 & 1.538 \\
\hline $07 / 27 / 00$ & $8: 47: 25$ & 59.9862 & 10.663 & 29.925 & 1.533 \\
\hline $07 / 27 / 00$ & $8: 47: 40$ & 60.2362 & 10.658 & 29.925 & 1.538 \\
\hline $07 / 27 / 00$ & $8: 47: 55$ & 60.4862 & 10.521 & 29.925 & 1.675 \\
\hline $07 / 27 / 00$ & $8: 48: 10$ & 60.7362 & 10.506 & 29.923 & 1.69 \\
\hline $07 / 27 / 00$ & 8:48:25 & 60.9862 & 10.532 & 29.923 & 1.664 \\
\hline $07 / 27 / 00$ & 8:48:40 & 61.2362 & 10.587 & 29.923 & 1.609 \\
\hline $07 / 27 / 00$ & 8:48:55 & 61.4862 & 10.626 & 29.923 & 1.57 \\
\hline $07 / 27 / 00$ & $8: 49: 10$ & 61.7362 & 10.655 & 29.921 & 1.541 \\
\hline $07 / 27 / 00$ & $8: 49: 25$ & 61.9862 & 10.682 & 29.923 & 1.514 \\
\hline $07 / 27 / 00$ & $8: 49: 40$ & 62.2362 & 10.699 & 29.923 & 1.497 \\
\hline $07 / 27 / 00$ & $8: 49: 55$ & 62.4862 & 10.714 & 29.923 & 1.482 \\
\hline $07 / 27 / 00$ & $8: 50: 10$ & 62.7362 & 10.723 & 29.923 & 1.473 \\
\hline $07 / 27 / 00$ & $8: 50: 25$ & 62.9862 & 10.72 & 29.923 & 1.476 \\
\hline $07 / 27 / 00$ & $8: 50: 40$ & 63.2362 & 10.718 & 29.923 & 1.478 \\
\hline $07 / 27 / 00$ & 8:50:55 & 63.4862 & 10.712 & 29.923 & 1.484 \\
\hline $07 / 27 / 00$ & $8: 51: 10$ & 63.7362 & 10.697 & 29.921 & 1.499 \\
\hline $07 / 27 / 00$ & $8: 51: 25$ & 63.9862 & 10.686 & 29.923 & 1.51 \\
\hline $07 / 27 / 00$ & $8: 51: 40$ & 64.2362 & 10.679 & 29.923 & 1.517 \\
\hline $07 / 27 / 00$ & $8: 51: 55$ & 64.4862 & 10.669 & 29.923 & 1.527 \\
\hline $07 / 27 / 00$ & 8:52:10 & 64.7362 & 10.656 & 29.923 & 1.54 \\
\hline $07 / 27 / 00$ & 8:52:25 & 64.9862 & 10.649 & 29.923 & 1.547 \\
\hline $07 / 27 / 00$ & 8:52:40 & 65.2362 & 10.645 & 29.921 & 1.551 \\
\hline $07 / 27 / 00$ & 8:52:55 & 65.4862 & 10.64 & 29.923 & 1.556 \\
\hline $07 / 27 / 00$ & $8: 53: 10$ & 65.7362 & 10.548 & 29.923 & 1.648 \\
\hline $07 / 27 / 00$ & $8: 53: 25$ & 65.9862 & 10.547 & 29.921 & 1.649 \\
\hline $07 / 27 / 00$ & 8:53:40 & 66.2362 & 10.545 & 29.921 & 1.651 \\
\hline $07 / 27 / 00$ & 8:53:55 & 66.4862 & 10.553 & 29.921 & 1.643 \\
\hline $07 / 27 / 00$ & $8: 54: 10$ & 66.7362 & 10.568 & 29.921 & 1.628 \\
\hline $07 / 27 / 00$ & $8: 54: 25$ & 66.9862 & 10.61 & 29.923 & 1.586 \\
\hline $07 / 27 / 00$ & $8: 54: 40$ & 67.2362 & 10.64 & 29.921 & 1.556 \\
\hline $07 / 27 / 00$ & $8: 54: 55$ & 67.4862 & 10.511 & 29.921 & 1.685 \\
\hline $07 / 27 / 00$ & 8:55:10 & 67.7362 & 10.548 & 29.921 & 1.648 \\
\hline $07 / 27 / 00$ & $8: 55: 25$ & 67.9862 & 10.597 & 29.923 & 1.599 \\
\hline $07 / 27 / 00$ & $8: 55: 40$ & 68.2362 & 10.635 & 29.921 & 1.561 \\
\hline $07 / 27 / 00$ & $8: 55: 55$ & 68.4862 & 10.648 & 29.921 & 1.548 \\
\hline $07 / 27 / 00$ & $8: 56: 10$ & 68.7362 & 10.656 & 29.921 & 1.54 \\
\hline $07 / 27 / 00$ & $8: 56: 25$ & 68.9862 & 10.655 & 29.923 & 1.541 \\
\hline $07 / 27 / 00$ & $8: 56: 40$ & 69.2362 & 10.653 & 29.921 & 1.543 \\
\hline $07 / 27 / 00$ & 8:56:55 & 69.4862 & 10.652 & 29.921 & 1.544 \\
\hline $07 / 27 / 00$ & $8: 57: 10$ & 69.7362 & 10.649 & 29.921 & 1.547 \\
\hline $07 / 27 / 00$ & $8: 57: 25$ & 69.9862 & 10.643 & 29.923 & 1.553 \\
\hline $07 / 27 / 00$ & $8: 57: 40$ & 70.2362 & 10.639 & 29.921 & 1.557 \\
\hline $07 / 27 / 00$ & $8: 57: 55$ & 70.4862 & 10.633 & 29.923 & 1.563 \\
\hline $07 / 27 / 00$ & 8:58:10 & 70.7362 & 10.629 & 29.921 & 1.567 \\
\hline $07 / 27 / 00$ & 8:58:25 & 70.9862 & 10.625 & 29.921 & 1.571 \\
\hline $07 / 27 / 00$ & 8:58:40 & 71.2362 & 10.619 & 29.921 & 1.577 \\
\hline $07 / 27 / 00$ & 8:58:55 & 71.4862 & 10.613 & 29.921 & 1.583 \\
\hline $07 / 27 / 00$ & $8: 59: 10$ & 71.7362 & 10.612 & 29.923 & 1.584 \\
\hline
\end{tabular}




\begin{tabular}{|c|c|c|c|c|c|}
\hline \multicolumn{6}{|c|}{ Appendix B (continued) } \\
\hline 07/27/00 & $8: 59: 25$ & 71.9862 & 10.553 & 29.921 & 1.643 \\
\hline $07 / 27 / 00$ & $8: 59: 40$ & 72.2362 & 10.47 & 29.921 & 1.726 \\
\hline $07 / 27 / 00$ & 8:59:55 & 72.4862 & 10.429 & 29.923 & 1.767 \\
\hline $07 / 27 / 00$ & 9:00:10 & 72.7362 & 10.344 & 29.923 & 1.852 \\
\hline $07 / 27 / 00$ & 9:00:25 & 72.9862 & 10.387 & 29.921 & 1.809 \\
\hline $07 / 27 / 00$ & $9: 00: 40$ & 73.2362 & 10.417 & 29.921 & 1.779 \\
\hline $07 / 27 / 00$ & $9: 00: 55$ & 73.4862 & 10.463 & 29.921 & 1.733 \\
\hline 07/27/00 & 9:01:10 & 73.7362 & 10.517 & 29.923 & 1.679 \\
\hline $07 / 27 / 00$ & $9: 01: 25$ & 73.9862 & 10.557 & 29.921 & 1.639 \\
\hline $07 / 27 / 00$ & 9:01:40 & 74.2362 & 10.591 & 29.923 & 1.605 \\
\hline 07/27/00 & 9:01:55 & 74.4862 & 10.614 & 29.923 & 1.582 \\
\hline $07 / 27 / 00$ & $9: 02: 10$ & 74.7362 & 10.635 & 29.923 & 1.561 \\
\hline $07 / 27 / 00$ & 9:02:25 & 74.9862 & 10.642 & 29.923 & 1.554 \\
\hline $07 / 27 / 00$ & 9:02:40 & 75.2362 & 10.603 & 29.921 & 1.593 \\
\hline $07 / 27 / 00$ & 9:02:55 & 75.4862 & 10.439 & 29.921 & 1.757 \\
\hline $07 / 27 / 00$ & 9:03:10 & 75.7362 & 10.352 & 29.923 & 1.844 \\
\hline $07 / 27 / 00$ & 9:03:25 & 75.9862 & 10.387 & 29.923 & 1.809 \\
\hline $07 / 27 / 00$ & 9:03:40 & 76.2362 & 10.413 & 29.923 & 1.783 \\
\hline $07 / 27 / 00$ & 9:03:55 & 76.4862 & 10.434 & 29.921 & 1.762 \\
\hline $07 / 27 / 00$ & 9:04:10 & 76.7362 & 10.453 & 29.923 & 1.743 \\
\hline $07 / 27 / 00$ & 9:04:25 & 76.9862 & 10.469 & 29.921 & 1.727 \\
\hline $07 / 27 / 00$ & 9:04:40 & 77.2362 & 10.482 & 29.921 & 1.714 \\
\hline $07 / 27 / 00$ & 9:04:55 & 77.4862 & 10.495 & 29.921 & 1.701 \\
\hline $07 / 27 / 00$ & 9:05:10 & 77.7362 & 10.505 & 29.921 & 1.691 \\
\hline $07 / 27 / 00$ & 9:05:25 & 77.9862 & 10.512 & 29.921 & 1.684 \\
\hline $07 / 27 / 00$ & $9: 05: 40$ & 78.2362 & 10.519 & 29.921 & 1.677 \\
\hline $07 / 27 / 00$ & 9:05:55 & 78.4862 & 10.525 & 29.923 & 1.671 \\
\hline $07 / 27 / 00$ & 9:06:10 & 78.7362 & 10.529 & 29.921 & 1.667 \\
\hline $07 / 27 / 00$ & 9:06:25 & 78.9862 & 10.535 & 29.921 & 1.661 \\
\hline $07 / 27 / 00$ & $9: 06: 40$ & 79.2362 & 10.541 & 29.921 & 1.655 \\
\hline $07 / 27 / 00$ & 9:06:55 & 79.4862 & 10.544 & 29.919 & 1.652 \\
\hline $07 / 27 / 00$ & 9:07:10 & 79.7362 & 10.59 & 29.921 & 1.606 \\
\hline $07 / 27 / 00$ & 9:07:25 & 79.9862 & 10.528 & 29.921 & 1.668 \\
\hline $07 / 27 / 00$ & 9:07:40 & 80.2362 & 10.531 & 29.921 & 1.665 \\
\hline $07 / 27 / 00$ & $9: 07: 55$ & 80.4862 & 10.521 & 29.921 & 1.675 \\
\hline $07 / 27 / 00$ & 9:08:10 & 80.7362 & 10.462 & 29.921 & 1.734 \\
\hline $07 / 27 / 00$ & 9:08:25 & 80.9862 & 10.309 & 29.921 & 1.887 \\
\hline $07 / 27 / 00$ & 9:08:40 & 81.2362 & 10.377 & 29.921 & 1.819 \\
\hline $07 / 27 / 00$ & 9:08:55 & 81.4862 & 10.385 & 29.921 & 1.811 \\
\hline $07 / 27 / 00$ & 9:09:10 & 81.7362 & 10.466 & 29.919 & 1.73 \\
\hline 07/27/00 & 9:09:25 & 81.9862 & 10.555 & 29.921 & 1.641 \\
\hline 07/27/00 & $9: 09: 40$ & 82.2362 & 10.64 & 29.919 & 1.556 \\
\hline 07/27/00 & 9:09:55 & 82.4862 & 10.678 & 29.919 & 1.518 \\
\hline 07/27/00 & $9: 10: 10$ & 82.7362 & 10.684 & 29.919 & 1.512 \\
\hline 07/27/00 & $9: 10: 25$ & 82.9862 & 10.625 & 29.921 & 1.571 \\
\hline 07/27/00 & $9: 10: 40$ & 83.2362 & 10.656 & 29.921 & 1.54 \\
\hline $07 / 27 / 00$ & $9: 10: 55$ & 83.4862 & 10.642 & 29.919 & 1.554 \\
\hline 07/27/00 & $9: 11: 10$ & 83.7362 & 10.625 & 29.919 & 1.571 \\
\hline 07/27/00 & $9: 11: 25$ & 83.9862 & 10.607 & 29.919 & 1.589 \\
\hline 07/27/00 & $9: 11: 40$ & 84.2362 & 10.594 & 29.919 & 1.602 \\
\hline 07/27/00 & $9: 11: 55$ & 84.4862 & 10.583 & 29.919 & 1.613 \\
\hline
\end{tabular}




\section{Appendix B (continued)}

\begin{tabular}{|c|c|c|c|c|c|}
\hline $07 / 27 / 00$ & 9:12:10 & 84.7362 & 10.573 & 29.919 & 1.623 \\
\hline $07 / 27 / 00$ & $9: 12: 25$ & 84.9862 & 10.563 & 29.919 & 1.633 \\
\hline $07 / 27 / 00$ & $9: 12: 40$ & 85.2362 & 10.555 & 29.919 & 1.641 \\
\hline $07 / 27 / 00$ & $9: 12: 55$ & 85.4862 & 10.551 & 29.919 & 1.645 \\
\hline $07 / 27 / 00$ & $9: 13: 10$ & 85.7362 & 10.551 & 29.917 & 1.645 \\
\hline $07 / 27 / 00$ & 9:13:25 & 85.9862 & 10.551 & 29.919 & 1.645 \\
\hline $07 / 27 / 00$ & $9: 13: 40$ & 86.2362 & 10.55 & 29.921 & 1.646 \\
\hline $07 / 27 / 00$ & 9:13:55 & 86.4862 & 10.545 & 29.919 & 1.651 \\
\hline $07 / 27 / 00$ & $9: 14: 10$ & 86.7362 & 10.544 & 29.919 & 1.652 \\
\hline $07 / 27 / 00$ & $9: 14: 25$ & 86.9862 & 10.541 & 29.919 & 1.655 \\
\hline $07 / 27 / 00$ & 9:14:40 & 87.2362 & 10.528 & 29.921 & 1.668 \\
\hline $07 / 27 / 00$ & 9:14:55 & 87.4862 & 10.46 & 29.919 & 1.736 \\
\hline $07 / 27 / 00$ & $9: 15: 10$ & 87.7362 & 10.352 & 29.917 & 1.844 \\
\hline $07 / 27 / 00$ & $9: 15: 25$ & 87.9862 & 10.522 & 29.919 & 1.674 \\
\hline $07 / 27 / 00$ & 9:15:40 & 88.2362 & 10.561 & 29.919 & 1.635 \\
\hline $07 / 27 / 00$ & 9:15:55 & 88.4862 & 10.64 & 29.919 & 1.556 \\
\hline $07 / 27 / 00$ & $9: 16: 10$ & 88.7362 & 10.629 & 29.921 & 1.567 \\
\hline $07 / 27 / 00$ & $9: 16: 25$ & 88.9862 & 10.627 & 29.921 & 1.569 \\
\hline $07 / 27 / 00$ & $9: 16: 40$ & 89.2362 & 10.676 & 29.919 & 1.52 \\
\hline $07 / 27 / 00$ & $9: 16: 55$ & 89.4862 & 10.701 & 29.917 & 1.495 \\
\hline $07 / 27 / 00$ & $9: 17: 10$ & 89.7362 & 10.73 & 29.919 & 1.466 \\
\hline $07 / 27 / 00$ & $9: 17: 25$ & 89.9862 & 10.751 & 29.919 & 1.445 \\
\hline $07 / 27 / 00$ & $9: 17: 40$ & 90.2362 & 10.767 & 29.919 & 1.429 \\
\hline $07 / 27 / 00$ & 9:17:55 & 90.4862 & 10.776 & 29.919 & 1.42 \\
\hline $07 / 27 / 00$ & $9: 18: 10$ & 90.7362 & 10.783 & 29.919 & 1.413 \\
\hline $07 / 27 / 00$ & $9: 18: 25$ & 90.9862 & 10.806 & 29.919 & 1.39 \\
\hline $07 / 27 / 00$ & 9:18:40 & 91.2362 & 10.777 & 29.919 & 1.419 \\
\hline $07 / 27 / 00$ & $9: 18: 55$ & 91.4862 & 10.769 & 29.919 & 1.427 \\
\hline $07 / 27 / 00$ & $9: 19: 10$ & 91.7362 & 10.761 & 29.919 & 1.435 \\
\hline $07 / 27 / 00$ & 9:19:25 & 91.9862 & 10.756 & 29.919 & 1.44 \\
\hline $07 / 27 / 00$ & $9: 19: 40$ & 92.2362 & 10.733 & 29.919 & 1.463 \\
\hline $07 / 27 / 00$ & 9:19:55 & 92.4862 & 10.72 & 29.919 & 1.476 \\
\hline $07 / 27 / 00$ & $9: 20: 10$ & 92.7362 & 10.727 & 29.917 & 1.469 \\
\hline $07 / 27 / 00$ & $9: 20: 25$ & 92.9862 & 10.708 & 29.919 & 1.488 \\
\hline $07 / 27 / 00$ & 9:20:40 & 93.2362 & 10.695 & 29.919 & 1.501 \\
\hline $07 / 27 / 00$ & 9:20:55 & 93.4862 & 10.679 & 29.917 & 1.517 \\
\hline $07 / 27 / 00$ & $9: 21: 10$ & 93.7362 & 10.685 & 29.919 & 1.511 \\
\hline $07 / 27 / 00$ & $9: 21: 25$ & 93.9862 & 10.686 & 29.919 & 1.51 \\
\hline $07 / 27 / 00$ & $9: 21: 40$ & 94.2362 & 10.689 & 29.919 & 1.507 \\
\hline $07 / 27 / 00$ & $9: 21: 55$ & 94.4862 & 10.686 & 29.919 & 1.51 \\
\hline $07 / 27 / 00$ & 9:22:10 & 94.7362 & 10.686 & 29.919 & 1.51 \\
\hline $07 / 27 / 00$ & 9:22:25 & 94.9862 & 10.678 & 29.919 & 1.518 \\
\hline $07 / 27 / 00$ & 9:22:40 & 95.2362 & 10.671 & 29.917 & 1.525 \\
\hline $07 / 27 / 00$ & $9: 22: 55$ & 95.4862 & 10.665 & 29.917 & 1.531 \\
\hline $07 / 27 / 00$ & $9: 23: 10$ & 95.7362 & 10.661 & 29.917 & 1.535 \\
\hline $07 / 27 / 00$ & $9: 23: 25$ & 95.9862 & 10.663 & 29.917 & 1.533 \\
\hline $07 / 27 / 00$ & 9:23:40 & 96.2362 & 10.645 & 29.917 & 1.551 \\
\hline $07 / 27 / 00$ & 9:23:55 & 96.4862 & 10.652 & 29.917 & 1.544 \\
\hline $07 / 27 / 00$ & $9: 24: 10$ & 96.7362 & 10.649 & 29.915 & 1.547 \\
\hline $07 / 27 / 00$ & $9: 24: 25$ & 96.9862 & 10.646 & 29.915 & 1.55 \\
\hline $07 / 27 / 00$ & $9: 24: 40$ & 97.2362 & 10.646 & 29.917 & 1.55 \\
\hline
\end{tabular}




\begin{tabular}{|c|c|c|c|c|c|}
\hline \multicolumn{6}{|c|}{ Appendix B (continued) } \\
\hline $07 / 27 / 00$ & $9: 24: 55$ & 97.4862 & 10.646 & 29.915 & 1.55 \\
\hline $07 / 27 / 00$ & $9: 25: 10$ & 97.7362 & 10.65 & 29.915 & 1.546 \\
\hline $07 / 27 / 00$ & $9: 25: 25$ & 97.9862 & 10.646 & 29.915 & 1.55 \\
\hline $07 / 27 / 00$ & $9: 25: 40$ & 98.2362 & 10.649 & 29.915 & 1.547 \\
\hline $07 / 27 / 00$ & $9: 25: 55$ & 98.4862 & 10.643 & 29.915 & 1.553 \\
\hline $07 / 27 / 00$ & $9: 26: 10$ & 98.7362 & 10.645 & 29.915 & 1.551 \\
\hline $07 / 27 / 00$ & $9: 26: 25$ & 98.9862 & 10.645 & 29.915 & 1.551 \\
\hline $07 / 27 / 00$ & $9: 26: 40$ & 99.2362 & 10.515 & 29.913 & 1.681 \\
\hline $07 / 27 / 00$ & $9: 26: 55$ & 99.4862 & 10.528 & 29.915 & 1.668 \\
\hline $07 / 27 / 00$ & $9: 27: 10$ & 99.7362 & 10.567 & 29.915 & 1.629 \\
\hline $07 / 27 / 00$ & $9: 27: 25$ & 99.9862 & 10.604 & 29.915 & 1.592 \\
\hline $07 / 27 / 00$ & $9: 27: 40$ & 100.2362 & 10.636 & 29.915 & 1.56 \\
\hline $07 / 27 / 00$ & $9: 27: 55$ & 100.4862 & 10.656 & 29.915 & 1.54 \\
\hline $07 / 27 / 00$ & $9: 28: 10$ & 100.7362 & 10.675 & 29.915 & 1.521 \\
\hline $07 / 27 / 00$ & $9: 28: 25$ & 100.9862 & 10.678 & 29.915 & 1.518 \\
\hline $07 / 27 / 00$ & 9:28:40 & 101.2362 & 10.685 & 29.915 & 1.511 \\
\hline $07 / 27 / 00$ & 9:28:55 & 101.4862 & 10.679 & 29.915 & 1.517 \\
\hline $07 / 27 / 00$ & $9: 29: 10$ & 101.7362 & 10.674 & 29.913 & 1.522 \\
\hline $07 / 27 / 00$ & $9: 29: 25$ & 101.9862 & 10.672 & 29.915 & 1.524 \\
\hline $07 / 27 / 00$ & $9: 29: 40$ & 102.2362 & 10.665 & 29.915 & 1.531 \\
\hline $07 / 27 / 00$ & $9: 29: 55$ & 102.4862 & 10.666 & 29.915 & 1.53 \\
\hline $07 / 27 / 00$ & 9:30:10 & 102.7362 & 10.661 & 29.915 & 1.535 \\
\hline $07 / 27 / 00$ & $9: 30: 25$ & 102.9862 & 10.662 & 29.915 & 1.534 \\
\hline $07 / 27 / 00$ & $9: 30: 40$ & 103.2362 & 10.659 & 29.915 & 1.537 \\
\hline $07 / 27 / 00$ & 9:30:55 & 103.4862 & 10.558 & 29.915 & 1.638 \\
\hline $07 / 27 / 00$ & $9: 31: 10$ & 103.7362 & 10.547 & 29.915 & 1.649 \\
\hline $07 / 27 / 00$ & $9: 31: 25$ & 103.9862 & 10.587 & 29.915 & 1.609 \\
\hline $07 / 27 / 00$ & $9: 31: 40$ & 104.2362 & 10.617 & 29.915 & 1.579 \\
\hline $07 / 27 / 00$ & $9: 31: 55$ & 104.4862 & 10.636 & 29.915 & 1.56 \\
\hline $07 / 27 / 00$ & 9:32:10 & 104.7362 & 10.648 & 29.915 & 1.548 \\
\hline $07 / 27 / 00$ & $9: 32: 25$ & 104.9862 & 10.646 & 29.915 & 1.55 \\
\hline $07 / 27 / 00$ & $9: 32: 40$ & 105.2362 & 10.652 & 29.915 & 1.544 \\
\hline $07 / 27 / 00$ & 9:32:55 & 105.4862 & 10.655 & 29.915 & 1.541 \\
\hline $07 / 27 / 00$ & $9: 33: 10$ & 105.7362 & 10.655 & 29.913 & 1.541 \\
\hline $07 / 27 / 00$ & 9:33:25 & 105.9862 & 10.65 & 29.913 & 1.546 \\
\hline $07 / 27 / 00$ & 9:33:40 & 106.2362 & 10.65 & 29.913 & 1.546 \\
\hline $07 / 27 / 00$ & $9: 33: 55$ & 106.4862 & 10.649 & 29.913 & 1.547 \\
\hline $07 / 27 / 00$ & $9: 34: 10$ & 106.7362 & 10.638 & 29.913 & 1.558 \\
\hline $07 / 27 / 00$ & $9: 34: 25$ & 106.9862 & 10.642 & 29.911 & 1.554 \\
\hline $07 / 27 / 00$ & $9: 34: 40$ & 107.2362 & 10.643 & 29.913 & 1.553 \\
\hline $07 / 27 / 00$ & $9: 34: 55$ & 107.4862 & 10.643 & 29.913 & 1.553 \\
\hline $07 / 27 / 00$ & 9:35:10 & 107.7362 & 10.648 & 29.913 & 1.548 \\
\hline $07 / 27 / 00$ & $9: 35: 25$ & 107.9862 & 10.522 & 29.913 & 1.674 \\
\hline $07 / 27 / 00$ & $9: 35: 40$ & 108.2362 & 10.534 & 29.913 & 1.662 \\
\hline $07 / 27 / 00$ & $9: 35: 55$ & 108.4862 & 10.584 & 29.911 & 1.612 \\
\hline $07 / 27 / 00$ & $9: 36: 10$ & 108.7362 & 10.613 & 29.911 & 1.583 \\
\hline $07 / 27 / 00$ & $9: 36: 25$ & 108.9862 & 10.636 & 29.911 & 1.56 \\
\hline $07 / 27 / 00$ & $9: 36: 40$ & 109.2362 & 10.648 & 29.911 & 1.548 \\
\hline $07 / 27 / 00$ & $9: 36: 55$ & 109.4862 & 10.655 & 29.913 & 1.541 \\
\hline $07 / 27 / 00$ & $9: 37: 10$ & 109.7362 & 10.666 & 29.913 & 1.53 \\
\hline $07 / 27 / 00$ & $9: 37: 25$ & 109.9862 & 10.663 & 29.911 & 1.533 \\
\hline
\end{tabular}




\section{Appendix B (continued)}

\begin{tabular}{|c|c|c|c|c|c|}
\hline $07 / 27 / 00$ & $9: 37: 40$ & 110.2362 & 10.668 & 29.911 & 1.528 \\
\hline $07 / 27 / 00$ & 9:37:55 & 110.4862 & 10.658 & 29.909 & 1.538 \\
\hline $07 / 27 / 00$ & 9:38:10 & 110.7362 & 10.659 & 29.911 & 1.537 \\
\hline $07 / 27 / 00$ & 9:38:25 & 110.9862 & 10.653 & 29.911 & 1.543 \\
\hline $07 / 27 / 00$ & 9:38:40 & 111.2362 & 10.648 & 29.909 & 1.548 \\
\hline $07 / 27 / 00$ & 9:38:55 & 111.4862 & 10.643 & 29.911 & 1.553 \\
\hline $07 / 27 / 00$ & 9:39:10 & 111.7362 & 10.642 & 29.911 & 1.554 \\
\hline $07 / 27 / 00$ & 9:39:25 & 111.9862 & 10.639 & 29.911 & 1.557 \\
\hline $07 / 27 / 00$ & 9:39:40 & 112.2362 & 10.639 & 29.909 & 1.557 \\
\hline $07 / 27 / 00$ & 9:39:55 & 112.4862 & 10.633 & 29.909 & 1.563 \\
\hline $07 / 27 / 00$ & 9:40:10 & 112.7362 & 10.492 & 29.911 & 1.704 \\
\hline $07 / 27 / 00$ & 9:40:25 & 112.9862 & 10.478 & 29.911 & 1.718 \\
\hline $07 / 27 / 00$ & 9:40:40 & 113.2362 & 10.529 & 29.909 & 1.667 \\
\hline 07/27/00 & 9:40:55 & 113.4862 & 10.568 & 29.911 & 1.628 \\
\hline $07 / 27 / 00$ & $9: 41: 10$ & 113.7362 & 10.609 & 29.911 & 1.587 \\
\hline $07 / 27 / 00$ & 9:41:25 & 113.9862 & 10.633 & 29.911 & 1.563 \\
\hline $07 / 27 / 00$ & 9:41:40 & 114.2362 & 10.65 & 29.913 & 1.546 \\
\hline $07 / 27 / 00$ & $9: 41: 55$ & 114.4862 & 10.642 & 29.911 & 1.554 \\
\hline $07 / 27 / 00$ & 9:42:10 & 114.7362 & 10.645 & 29.911 & 1.551 \\
\hline $07 / 27 / 00$ & 9:42:25 & 114.9862 & 10.648 & 29.911 & 1.548 \\
\hline $07 / 27 / 00$ & 9:42:40 & 115.2362 & 10.648 & 29.913 & 1.548 \\
\hline $07 / 27 / 00$ & 9:42:55 & 115.4862 & 10.649 & 29.911 & 1.547 \\
\hline $07 / 27 / 00$ & 9:43:10 & 115.7362 & 10.646 & 29.911 & 1.55 \\
\hline $07 / 27 / 00$ & 9:43:25 & 115.9862 & 10.649 & 29.911 & 1.547 \\
\hline $07 / 27 / 00$ & 9:43:40 & 116.2362 & 10.649 & 29.913 & 1.547 \\
\hline $07 / 27 / 00$ & 9:43:55 & 116.4862 & 10.645 & 29.911 & 1.551 \\
\hline 07/27/00 & 9:44:10 & 116.7362 & 10.643 & 29.911 & 1.553 \\
\hline 07/27/00 & 9:44:25 & 116.9862 & 10.64 & 29.911 & 1.556 \\
\hline 07/27/00 & 9:44:40 & 117.2362 & 10.642 & 29.913 & 1.554 \\
\hline $07 / 27 / 00$ & 9:44:55 & 117.4862 & 10.635 & 29.911 & 1.561 \\
\hline 07/27/00 & 9:45:10 & 117.7362 & 10.587 & 29.911 & 1.609 \\
\hline 07/27/00 & 9:45:25 & 117.9862 & 10.489 & 29.913 & 1.707 \\
\hline 07/27/00 & 9:45:40 & 118.2362 & 10.519 & 29.911 & 1.677 \\
\hline 07/27/00 & 9:45:55 & 118.4862 & 10.56 & 29.913 & 1.636 \\
\hline 07/27/00 & 9:46:10 & 118.7362 & 10.596 & 29.911 & 1.6 \\
\hline $07 / 27 / 00$ & 9:46:25 & 118.9862 & 10.616 & 29.911 & 1.58 \\
\hline 07/27/00 & 9:46:40 & 119.2362 & 10.632 & 29.911 & 1.564 \\
\hline 07/27/00 & 9:46:55 & 119.4862 & 10.639 & 29.911 & 1.557 \\
\hline 07/27/00 & 9:47:10 & 119.7362 & 10.636 & 29.911 & 1.56 \\
\hline $07 / 27 / 00$ & 9:47:25 & 119.9862 & 10.64 & 29.913 & 1.556 \\
\hline 07/27/00 & 9:47:40 & 120.2362 & 10.635 & 29.911 & 1.561 \\
\hline $07 / 27 / 00$ & 9:47:55 & 120.4862 & 10.625 & 29.911 & 1.571 \\
\hline $07 / 27 / 00$ & 9:48:10 & 120.7362 & 10.622 & 29.911 & 1.574 \\
\hline 07/27/00 & 9:48:25 & 120.9862 & 10.512 & 29.911 & 1.684 \\
\hline 07/27/00 & 9:48:40 & 121.2362 & 10.36 & 29.911 & 1.836 \\
\hline 07/27/00 & 9:48:55 & 121.4862 & 10.362 & 29.911 & 1.834 \\
\hline 07/27/00 & 9:49:10 & 121.7362 & 10.383 & 29.911 & 1.813 \\
\hline $07 / 27 / 00$ & 9:49:25 & 121.9862 & 10.393 & 29.911 & 1.803 \\
\hline $07 / 27 / 00$ & 9:49:40 & 122.2362 & 10.403 & 29.913 & 1.793 \\
\hline 07/27/00 & 9:49:55 & 122.4862 & 10.416 & 29.925 & 1.78 \\
\hline 07/27/00 & 9:50:10 & 122.7362 & 10.434 & 29.915 & 1.762 \\
\hline
\end{tabular}




\begin{tabular}{|c|c|c|c|c|c|}
\hline \multicolumn{6}{|c|}{ Appendix B (continued) } \\
\hline $07 / 27 / 00$ & $9: 50: 25$ & 122.9862 & 10.449 & 29.913 & 1.747 \\
\hline $07 / 27 / 00$ & $9: 50: 40$ & 123.2362 & 10.463 & 29.911 & 1.733 \\
\hline $07 / 27 / 00$ & $9: 50: 55$ & 123.4862 & 10.468 & 29.915 & 1.728 \\
\hline $07 / 27 / 00$ & $9: 51: 10$ & 123.7362 & 10.466 & 29.911 & 1.73 \\
\hline $07 / 27 / 00$ & $9: 51: 25$ & 123.9862 & 10.38 & 29.911 & 1.816 \\
\hline $07 / 27 / 00$ & $9: 51: 40$ & 124.2362 & 10.272 & 29.909 & 1.924 \\
\hline $07 / 27 / 00$ & $9: 51: 55$ & 124.4862 & 10.182 & 29.911 & 2.014 \\
\hline $07 / 27 / 00$ & $9: 52: 10$ & 124.7362 & 10.102 & 29.911 & 2.094 \\
\hline $07 / 27 / 00$ & $9: 52: 25$ & 124.9862 & 10.031 & 29.911 & 2.165 \\
\hline $07 / 27 / 00$ & $9: 52: 40$ & 125.2362 & 9.974 & 29.909 & 2.222 \\
\hline $07 / 27 / 00$ & $9: 52: 55$ & 125.4862 & 9.92 & 29.909 & 2.276 \\
\hline $07 / 27 / 00$ & $9: 53: 10$ & 125.7362 & 9.876 & 29.909 & 2.32 \\
\hline $07 / 27 / 00$ & 9:53:25 & 125.9862 & 9.832 & 29.911 & 2.364 \\
\hline $07 / 27 / 00$ & $9: 53: 40$ & 126.2362 & 9.795 & 29.909 & 2.401 \\
\hline $07 / 27 / 00$ & $9: 53: 55$ & 126.4862 & 9.762 & 29.909 & 2.434 \\
\hline $07 / 27 / 00$ & $9: 54: 10$ & 126.7362 & 9.732 & 29.909 & 2.464 \\
\hline $07 / 27 / 00$ & $9: 54: 25$ & 126.9862 & 9.703 & 29.911 & 2.493 \\
\hline $07 / 27 / 00$ & $9: 54: 40$ & 127.2362 & 9.678 & 29.909 & 2.518 \\
\hline $07 / 27 / 00$ & $9: 54: 55$ & 127.4862 & 9.655 & 29.909 & 2.541 \\
\hline $07 / 27 / 00$ & $9: 55: 10$ & 127.7362 & 9.635 & 29.909 & 2.561 \\
\hline $07 / 27 / 00$ & $9: 55: 25$ & 127.9862 & 9.615 & 29.909 & 2.581 \\
\hline $07 / 27 / 00$ & $9: 55: 40$ & 128.2362 & 9.599 & 29.917 & 2.597 \\
\hline $07 / 27 / 00$ & $9: 55: 55$ & 128.4862 & 9.582 & 29.907 & 2.614 \\
\hline $07 / 27 / 00$ & $9: 56: 10$ & 128.7362 & 9.566 & 29.909 & 2.63 \\
\hline $07 / 27 / 00$ & 9:56:25 & 128.9862 & 9.552 & 29.909 & 2.644 \\
\hline $07 / 27 / 00$ & $9: 56: 40$ & 129.2362 & 9.49 & 29.909 & 2.706 \\
\hline $07 / 27 / 00$ & $9: 56: 55$ & 129.4862 & 9.349 & 29.907 & 2.847 \\
\hline $07 / 27 / 00$ & $9: 57: 10$ & 129.7362 & 9.285 & 29.909 & 2.911 \\
\hline $07 / 27 / 00$ & $9: 57: 25$ & 129.9862 & 9.104 & 29.909 & 3.092 \\
\hline $07 / 27 / 00$ & $9: 57: 40$ & 130.2362 & 9.154 & 29.909 & 3.042 \\
\hline $07 / 27 / 00$ & $9: 57: 55$ & 130.4862 & 8.902 & 29.909 & 3.294 \\
\hline $07 / 27 / 00$ & $9: 58: 10$ & 130.7362 & 8.968 & 29.907 & 3.228 \\
\hline $07 / 27 / 00$ & 9:58:25 & 130.9862 & 9.019 & 29.907 & 3.177 \\
\hline $07 / 27 / 00$ & $9: 58: 40$ & 131.2362 & 9.061 & 29.909 & 3.135 \\
\hline $07 / 27 / 00$ & $9: 58: 55$ & 131.4862 & 9.095 & 29.909 & 3.101 \\
\hline $07 / 27 / 00$ & 9:59:10 & 131.7362 & 9.122 & 29.909 & 3.074 \\
\hline $07 / 27 / 00$ & $9: 59: 25$ & 131.9862 & 9.148 & 29.909 & 3.048 \\
\hline $07 / 27 / 00$ & $9: 59: 40$ & 132.2362 & 9.169 & 29.909 & 3.027 \\
\hline $07 / 27 / 00$ & 9:59:55 & 132.4862 & 9.184 & 29.909 & 3.012 \\
\hline $07 / 27 / 00$ & 10:00:10 & 132.7362 & 9.199 & 29.909 & 2.997 \\
\hline $07 / 27 / 00$ & $10: 00: 25$ & 132.9862 & 9.21 & 29.909 & 2.986 \\
\hline $07 / 27 / 00$ & $10: 00: 40$ & 133.2362 & 9.219 & 29.909 & 2.977 \\
\hline $07 / 27 / 00$ & 10:00:55 & 133.4862 & 9.226 & 29.909 & 2.97 \\
\hline $07 / 27 / 00$ & 10:01:10 & 133.7362 & 9.233 & 29.909 & 2.963 \\
\hline $07 / 27 / 00$ & 10:01:25 & 133.9862 & 9.236 & 29.909 & 2.96 \\
\hline $07 / 27 / 00$ & $10: 01: 40$ & 134.2362 & 9.241 & 29.911 & 2.955 \\
\hline $07 / 27 / 00$ & $10: 01: 55$ & 134.4862 & 9.243 & 29.909 & 2.953 \\
\hline $07 / 27 / 00$ & $10: 02: 10$ & 134.7362 & 9.549 & 29.909 & 2.647 \\
\hline $07 / 27 / 00$ & 10:02:25 & 134.9862 & 9.531 & 29.909 & 2.665 \\
\hline $07 / 27 / 00$ & $10: 02: 40$ & 135.2362 & 9.698 & 29.909 & 2.498 \\
\hline $07 / 27 / 00$ & $10: 02: 55$ & 135.4862 & 9.878 & 29.909 & 2.318 \\
\hline
\end{tabular}




\section{Appendix B (continued)}

\begin{tabular}{|c|c|c|c|c|c|}
\hline $07 / 27 / 00$ & $10: 03: 10$ & 135.7362 & 9.806 & 29.909 & 2.39 \\
\hline $07 / 27 / 00$ & 10:03:25 & 135.9862 & 9.739 & 29.909 & 2.457 \\
\hline $07 / 27 / 00$ & 10:03:40 & 136.2362 & 9.677 & 29.911 & 2.519 \\
\hline $07 / 27 / 00$ & 10:03:55 & 136.4862 & 9.629 & 29.911 & 2.567 \\
\hline $07 / 27 / 00$ & 10:04:10 & 136.7362 & 9.589 & 29.909 & 2.607 \\
\hline $07 / 27 / 00$ & 10:04:25 & 136.9862 & 9.554 & 29.909 & 2.642 \\
\hline $07 / 27 / 00$ & 10:04:40 & 137.2362 & 9.524 & 29.911 & 2.672 \\
\hline $07 / 27 / 00$ & 10:04:55 & 137.4862 & 9.5 & 29.909 & 2.696 \\
\hline $07 / 27 / 00$ & 10:05:10 & 137.7362 & 9.475 & 29.909 & 2.721 \\
\hline $07 / 27 / 00$ & 10:05:25 & 137.9862 & 9.454 & 29.909 & 2.742 \\
\hline $07 / 27 / 00$ & 10:05:40 & 138.2362 & 9.436 & 29.909 & 2.76 \\
\hline $07 / 27 / 00$ & 10:05:55 & 138.4862 & 9.422 & 29.911 & 2.774 \\
\hline $07 / 27 / 00$ & 10:06:10 & 138.7362 & 9.405 & 29.911 & 2.791 \\
\hline $07 / 27 / 00$ & 10:06:25 & 138.9862 & 9.393 & 29.909 & 2.803 \\
\hline $07 / 27 / 00$ & 10:06:40 & 139.2362 & 9.382 & 29.909 & 2.814 \\
\hline $07 / 27 / 00$ & 10:06:55 & 139.4862 & 9.369 & 29.909 & 2.827 \\
\hline $07 / 27 / 00$ & 10:07:10 & 139.7362 & 9.36 & 29.909 & 2.836 \\
\hline $07 / 27 / 00$ & 10:07:25 & 139.9862 & 9.351 & 29.909 & 2.845 \\
\hline $07 / 27 / 00$ & 10:07:40 & 140.2362 & 9.344 & 29.911 & 2.852 \\
\hline $07 / 27 / 00$ & 10:07:55 & 140.4862 & 9.337 & 29.911 & 2.859 \\
\hline $07 / 27 / 00$ & 10:08:10 & 140.7362 & 9.331 & 29.909 & 2.865 \\
\hline $07 / 27 / 00$ & 10:08:25 & 140.9862 & 9.323 & 29.911 & 2.873 \\
\hline $07 / 27 / 00$ & 10:08:40 & 141.2362 & 9.318 & 29.911 & 2.878 \\
\hline $07 / 27 / 00$ & 10:08:55 & 141.4862 & 9.314 & 29.909 & 2.882 \\
\hline $07 / 27 / 00$ & 10:09:10 & 141.7362 & 9.308 & 29.909 & 2.888 \\
\hline $07 / 27 / 00$ & 10:09:25 & 141.9862 & 9.305 & 29.909 & 2.891 \\
\hline $07 / 27 / 00$ & 10:09:40 & 142.2362 & 9.301 & 29.911 & 2.895 \\
\hline $07 / 27 / 00$ & 10:09:55 & 142.4862 & 9.295 & 29.909 & 2.901 \\
\hline $07 / 27 / 00$ & 10:10:10 & 142.7362 & 9.291 & 29.929 & 2.905 \\
\hline $07 / 27 / 00$ & $10: 10: 25$ & 142.9862 & 9.287 & 29.933 & 2.909 \\
\hline $07 / 27 / 00$ & $10: 10: 40$ & 143.2362 & 9.282 & 29.915 & 2.914 \\
\hline $07 / 27 / 00$ & 10:10:55 & 143.4862 & 9.278 & 29.913 & 2.918 \\
\hline $07 / 27 / 00$ & 10:11:10 & 143.7362 & 9.275 & 29.911 & 2.921 \\
\hline $07 / 27 / 00$ & 10:11:25 & 143.9862 & 9.274 & 29.911 & 2.922 \\
\hline $07 / 27 / 00$ & $10: 11: 40$ & 144.2362 & 9.269 & 29.909 & 2.927 \\
\hline $07 / 27 / 00$ & 10:11:55 & 144.4862 & 9.266 & 29.909 & 2.93 \\
\hline $07 / 27 / 00$ & 10:12:10 & 144.7362 & 9.262 & 29.909 & 2.934 \\
\hline $07 / 27 / 00$ & 10:12:25 & 144.9862 & 9.261 & 29.909 & 2.935 \\
\hline $07 / 27 / 00$ & 10:12:40 & 145.2362 & 9.256 & 29.909 & 2.94 \\
\hline $07 / 27 / 00$ & $10: 12: 55$ & 145.4862 & 9.255 & 29.909 & 2.941 \\
\hline $07 / 27 / 00$ & 10:13:10 & 145.7362 & 9.253 & 29.909 & 2.943 \\
\hline $07 / 27 / 00$ & 10:13:25 & 145.9862 & 9.251 & 29.907 & 2.945 \\
\hline $07 / 27 / 00$ & 10:13:40 & 146.2362 & 9.245 & 29.905 & 2.951 \\
\hline $07 / 27 / 00$ & 10:13:55 & 146.4862 & 9.242 & 29.907 & 2.954 \\
\hline $07 / 27 / 00$ & 10:14:10 & 146.7362 & 9.241 & 29.909 & 2.955 \\
\hline $07 / 27 / 00$ & $10: 14: 25$ & 146.9862 & 9.239 & 29.907 & 2.957 \\
\hline $07 / 27 / 00$ & $10: 14: 40$ & 147.2362 & 9.236 & 29.907 & 2.96 \\
\hline $07 / 27 / 00$ & 10:14:55 & 147.4862 & 9.235 & 29.909 & 2.961 \\
\hline $07 / 27 / 00$ & $10: 15: 10$ & 147.7362 & 9.23 & 29.909 & 2.966 \\
\hline $07 / 27 / 00$ & 10:15:25 & 147.9862 & 9.23 & 29.909 & 2.966 \\
\hline $07 / 27 / 00$ & $10: 15: 40$ & 148.2362 & 9.228 & 29.907 & 2.968 \\
\hline
\end{tabular}




\begin{tabular}{|c|c|c|c|c|c|}
\hline \multicolumn{6}{|c|}{ Appendix B (continued) } \\
\hline 07/27/00 & $10: 15: 55$ & 148.4862 & 9.225 & 29.909 & 2.971 \\
\hline 07/27/00 & $10: 16: 10$ & 148.7362 & 9.223 & 29.907 & 2.973 \\
\hline 07/27/00 & $10: 16: 25$ & 148.9862 & 9.222 & 29.909 & 2.974 \\
\hline 07/27/00 & $10: 16: 40$ & 149.2362 & 9.219 & 29.907 & 2.977 \\
\hline 07/27/00 & $10: 16: 55$ & 149.4862 & 9.219 & 29.907 & 2.977 \\
\hline 07/27/00 & $10: 17: 10$ & 149.7362 & 9.217 & 29.911 & 2.979 \\
\hline 07/27/00 & $10: 17: 25$ & 149.9862 & 9.213 & 29.909 & 2.983 \\
\hline 07/27/00 & $10: 17: 40$ & 150.2362 & 9.212 & 29.919 & 2.984 \\
\hline $07 / 27 / 00$ & $10: 17: 55$ & 150.4862 & 9.21 & 29.921 & 2.986 \\
\hline 07/27/00 & $10: 18: 10$ & 150.7362 & 9.209 & 29.911 & 2.987 \\
\hline 07/27/00 & $10: 18: 25$ & 150.9862 & 9.207 & 29.911 & 2.989 \\
\hline 07/27/00 & $10: 18: 40$ & 151.2362 & 9.206 & 29.909 & 2.99 \\
\hline 07/27/00 & $10: 18: 55$ & 151.4862 & 9.205 & 29.909 & 2.991 \\
\hline $07 / 27 / 00$ & $10: 19: 10$ & 151.7362 & 9.2 & 29.909 & 2.996 \\
\hline 07/27/00 & 10:19:25 & 151.9862 & 9.2 & 29.909 & 2.996 \\
\hline $07 / 27 / 00$ & $10: 19: 40$ & 152.2362 & 9.199 & 29.909 & 2.997 \\
\hline 07/27/00 & $10: 19: 55$ & 152.4862 & 9.196 & 29.909 & 3 \\
\hline 07/27/00 & $10: 20: 10$ & 152.7362 & 9.194 & 29.909 & 3.002 \\
\hline $07 / 27 / 00$ & $10: 20: 25$ & 152.9862 & 9.193 & 29.909 & 3.003 \\
\hline $07 / 27 / 00$ & $10: 20: 40$ & 153.2362 & 9.192 & 29.909 & 3.004 \\
\hline 07/27/00 & $10: 20: 55$ & 153.4862 & 9.19 & 29.909 & 3.006 \\
\hline $07 / 27 / 00$ & $10: 21: 10$ & 153.7362 & 9.189 & 29.909 & 3.007 \\
\hline $07 / 27 / 00$ & $10: 21: 25$ & 153.9862 & 9.186 & 29.909 & 3.01 \\
\hline $07 / 27 / 00$ & $10: 21: 40$ & 154.2362 & 9.184 & 29.907 & 3.012 \\
\hline $07 / 27 / 00$ & $10: 21: 55$ & 154.4862 & 9.183 & 29.907 & 3.013 \\
\hline $07 / 27 / 00$ & $10: 22: 10$ & 154.7362 & 9.246 & 29.907 & 2.95 \\
\hline $07 / 27 / 00$ & $10: 22: 25$ & 154.9862 & 9.277 & 29.909 & 2.919 \\
\hline $07 / 27 / 00$ & $10: 22: 40$ & 155.2362 & 9.261 & 29.909 & 2.935 \\
\hline 07/27/00 & $10: 22: 55$ & 155.4862 & 9.251 & 29.909 & 2.945 \\
\hline 07/27/00 & $10: 23: 10$ & 155.7362 & 9.239 & 29.909 & 2.957 \\
\hline 07/27/00 & $10: 23: 25$ & 155.9862 & 9.14 & 29.909 & 3.056 \\
\hline 07/27/00 & $10: 23: 40$ & 156.2362 & 9.169 & 29.909 & 3.027 \\
\hline $07 / 27 / 00$ & $10: 23: 55$ & 156.4862 & 9.222 & 29.907 & 2.974 \\
\hline $07 / 27 / 00$ & $10: 24: 10$ & 156.7362 & 9.269 & 29.909 & 2.927 \\
\hline 07/27/00 & $10: 24: 25$ & 156.9862 & 9.307 & 29.909 & 2.889 \\
\hline 07/27/00 & $10: 24: 40$ & 157.2362 & 9.334 & 29.909 & 2.862 \\
\hline 07/27/00 & $10: 24: 55$ & 157.4862 & 9.351 & 29.907 & 2.845 \\
\hline 07/27/00 & $10: 25: 10$ & 157.7362 & 9.324 & 29.907 & 2.872 \\
\hline 07/27/00 & $10: 25: 25$ & 157.9862 & 9.304 & 29.909 & 2.892 \\
\hline $07 / 27 / 00$ & $10: 25: 40$ & 158.2362 & 9.284 & 29.909 & 2.912 \\
\hline $07 / 27 / 00$ & $10: 25: 55$ & 158.4862 & 9.269 & 29.907 & 2.927 \\
\hline 07/27/00 & $10: 26: 10$ & 158.7362 & 9.256 & 29.907 & 2.94 \\
\hline 07/27/00 & $10: 26: 25$ & 158.9862 & 9.245 & 29.907 & 2.951 \\
\hline 07/27/00 & $10: 26: 40$ & 159.2362 & 9.163 & 29.907 & 3.033 \\
\hline 07/27/00 & $10: 26: 55$ & 159.4862 & 9.173 & 29.907 & 3.023 \\
\hline 07/27/00 & $10: 27: 10$ & 159.7362 & 9.22 & 29.909 & 2.976 \\
\hline 07/27/00 & $10: 27: 25$ & 159.9862 & 9.215 & 29.909 & 2.981 \\
\hline 07/27/00 & $10: 27: 40$ & 160.2362 & 9.207 & 29.907 & 2.989 \\
\hline 07/27/00 & $10: 27: 55$ & 160.4862 & 9.199 & 29.907 & 2.997 \\
\hline 07/27/00 & $10: 28: 10$ & 160.7362 & 9.194 & 29.909 & 3.002 \\
\hline $07 / 27 / 00$ & $10: 28: 25$ & 160.9862 & 9.19 & 29.907 & 3.006 \\
\hline
\end{tabular}




\section{Appendix B (continued)}

\begin{tabular}{|c|c|c|c|c|c|}
\hline $07 / 27 / 00$ & $10: 28: 40$ & 161.2362 & 9.186 & 29.909 & 3.01 \\
\hline $07 / 27 / 00$ & 10:28:55 & 161.4862 & 9.18 & 29.909 & 3.016 \\
\hline $07 / 27 / 00$ & 10:29:10 & 161.7362 & 9.177 & 29.907 & 3.019 \\
\hline $07 / 27 / 00$ & 10:29:25 & 161.9862 & 9.107 & 29.909 & 3.089 \\
\hline $07 / 27 / 00$ & 10:29:40 & 162.2362 & 9.085 & 29.909 & 3.111 \\
\hline $07 / 27 / 00$ & 10:29:55 & 162.4862 & 9.151 & 29.907 & 3.045 \\
\hline $07 / 27 / 00$ & 10:30:10 & 162.7362 & 9.18 & 29.907 & 3.016 \\
\hline $07 / 27 / 00$ & 10:30:25 & 162.9862 & 9.171 & 29.905 & 3.025 \\
\hline $07 / 27 / 00$ & 10:30:40 & 163.2362 & 9.167 & 29.905 & 3.029 \\
\hline $07 / 27 / 00$ & 10:30:55 & 163.4862 & 9.163 & 29.907 & 3.033 \\
\hline $07 / 27 / 00$ & 10:31:10 & 163.7362 & 9.158 & 29.907 & 3.038 \\
\hline $07 / 27 / 00$ & 10:31:25 & 163.9862 & 9.156 & 29.905 & 3.04 \\
\hline $07 / 27 / 00$ & 10:31:40 & 164.2362 & 9.153 & 29.905 & 3.043 \\
\hline $07 / 27 / 00$ & 10:31:55 & 164.4862 & 9.15 & 29.907 & 3.046 \\
\hline $07 / 27 / 00$ & 10:32:10 & 164.7362 & 9.147 & 29.905 & 3.049 \\
\hline $07 / 27 / 00$ & 10:32:25 & 164.9862 & 9.145 & 29.905 & 3.051 \\
\hline $07 / 27 / 00$ & 10:32:40 & 165.2362 & 9.032 & 29.905 & 3.164 \\
\hline $07 / 27 / 00$ & 10:32:55 & 165.4862 & 9.099 & 29.903 & 3.097 \\
\hline $07 / 27 / 00$ & 10:33:10 & 165.7362 & 9.151 & 29.905 & 3.045 \\
\hline $07 / 27 / 00$ & 10:33:25 & 165.9862 & 9.17 & 29.903 & 3.026 \\
\hline $07 / 27 / 00$ & 10:33:40 & 166.2362 & 9.151 & 29.903 & 3.045 \\
\hline $07 / 27 / 00$ & 10:33:55 & 166.4862 & 9.148 & 29.903 & 3.048 \\
\hline $07 / 27 / 00$ & 10:34:10 & 166.7362 & 9.144 & 29.903 & 3.052 \\
\hline $07 / 27 / 00$ & 10:34:25 & 166.9862 & 9.141 & 29.903 & 3.055 \\
\hline $07 / 27 / 00$ & 10:34:40 & 167.2362 & 9.137 & 29.903 & 3.059 \\
\hline $07 / 27 / 00$ & 10:34:55 & 167.4862 & 9.135 & 29.903 & 3.061 \\
\hline $07 / 27 / 00$ & 10:35:10 & 167.7362 & 9.133 & 29.903 & 3.063 \\
\hline $07 / 27 / 00$ & 10:35:25 & 167.9862 & 9.082 & 29.903 & 3.114 \\
\hline $07 / 27 / 00$ & 10:35:40 & 168.2362 & 8.983 & 29.903 & 3.213 \\
\hline $07 / 27 / 00$ & 10:35:55 & 168.4862 & 9.032 & 29.903 & 3.164 \\
\hline $07 / 27 / 00$ & 10:36:10 & 168.7362 & 9.099 & 29.901 & 3.097 \\
\hline $07 / 27 / 00$ & 10:36:25 & 168.9862 & 9.153 & 29.903 & 3.043 \\
\hline $07 / 27 / 00$ & 10:36:40 & 169.2362 & 9.179 & 29.903 & 3.017 \\
\hline $07 / 27 / 00$ & $10: 36: 55$ & 169.4862 & 9.17 & 29.903 & 3.026 \\
\hline $07 / 27 / 00$ & 10:37:10 & 169.7362 & 9.157 & 29.903 & 3.039 \\
\hline $07 / 27 / 00$ & 10:37:25 & 169.9862 & 9.151 & 29.903 & 3.045 \\
\hline $07 / 27 / 00$ & 10:37:40 & 170.2362 & 9.145 & 29.903 & 3.051 \\
\hline $07 / 27 / 00$ & 10:37:55 & 170.4862 & 9.141 & 29.901 & 3.055 \\
\hline $07 / 27 / 00$ & 10:38:10 & 170.7362 & 9.135 & 29.903 & 3.061 \\
\hline $07 / 27 / 00$ & 10:38:25 & 170.9862 & 9.039 & 29.903 & 3.157 \\
\hline $07 / 27 / 00$ & 10:38:40 & 171.2362 & 8.896 & 29.903 & 3.3 \\
\hline $07 / 27 / 00$ & 10:38:55 & 171.4862 & 8.872 & 29.903 & 3.324 \\
\hline $07 / 27 / 00$ & 10:39:10 & 171.7362 & 8.912 & 29.905 & 3.284 \\
\hline $07 / 27 / 00$ & 10:39:25 & 171.9862 & 8.999 & 29.903 & 3.197 \\
\hline $07 / 27 / 00$ & 10:39:40 & 172.2362 & 9.05 & 29.907 & 3.146 \\
\hline $07 / 27 / 00$ & 10:39:55 & 172.4862 & 9.108 & 29.905 & 3.088 \\
\hline $07 / 27 / 00$ & 10:40:10 & 172.7362 & 9.148 & 29.905 & 3.048 \\
\hline $07 / 27 / 00$ & 10:40:25 & 172.9862 & 9.144 & 29.905 & 3.052 \\
\hline $07 / 27 / 00$ & 10:40:40 & 173.2362 & 9.134 & 29.905 & 3.062 \\
\hline 07/27/00 & 10:40:55 & 173.4862 & 9.127 & 29.905 & 3.069 \\
\hline $07 / 27 / 00$ & 10:41:10 & 173.7362 & 9.121 & 29.907 & 3.075 \\
\hline
\end{tabular}




\section{Appendix B (continued)}

\begin{tabular}{|c|c|c|c|c|c|}
\hline $07 / 27 / 00$ & $10: 41: 25$ & 173.9862 & 9.115 & 29.907 & 3.081 \\
\hline 07/27/00 & 10:41:40 & 174.2362 & 9.111 & 29.905 & 3.085 \\
\hline 07/27/00 & 10:41:55 & 174.4862 & 9.081 & 29.907 & 3.115 \\
\hline 07/27/00 & 10:42:10 & 174.7362 & 8.993 & 29.907 & 3.203 \\
\hline 07/27/00 & $10: 42: 25$ & 174.9862 & 9.063 & 29.905 & 3.133 \\
\hline 07/27/00 & 10:42:40 & 175.2362 & 9.118 & 29.907 & 3.078 \\
\hline 07/27/00 & 10:42:55 & 175.4862 & 9.109 & 29.905 & 3.087 \\
\hline 07/27/00 & 10:43:10 & 175.7362 & 9.105 & 29.905 & 3.091 \\
\hline 07/27/00 & 10:43:25 & 175.9862 & 9.102 & 29.905 & 3.094 \\
\hline 07/27/00 & 10:43:40 & 176.2362 & 9.098 & 29.907 & 3.098 \\
\hline 07/27/00 & 10:43:55 & 176.4862 & 9.095 & 29.907 & 3.101 \\
\hline 07/27/00 & 10:44:10 & 176.7362 & 9.094 & 29.907 & 3.102 \\
\hline 07/27/00 & 10:44:25 & 176.9862 & 9.092 & 29.907 & 3.104 \\
\hline 07/27/00 & 10:44:40 & 177.2362 & 9.091 & 29.907 & 3.105 \\
\hline 07/27/00 & 10:44:55 & 177.4862 & 9.088 & 29.909 & 3.108 \\
\hline 07/27/00 & $10: 45: 10$ & 177.7362 & 9.086 & 29.907 & 3.11 \\
\hline 07/27/00 & $10: 45: 25$ & 177.9862 & 9.012 & 29.905 & 3.184 \\
\hline 07/27/00 & 10:45:40 & 178.2362 & 8.935 & 29.907 & 3.261 \\
\hline 07/27/00 & 10:45:55 & 178.4862 & 9.026 & 29.905 & 3.17 \\
\hline 07/27/00 & 10:46:10 & 178.7362 & 9.092 & 29.907 & 3.104 \\
\hline 07/27/00 & 10:46:25 & 178.9862 & 9.124 & 29.907 & 3.072 \\
\hline 07/27/00 & 10:46:40 & 179.2362 & 9.114 & 29.907 & 3.082 \\
\hline 07/27/00 & $10: 46: 55$ & 179.4862 & 9.108 & 29.907 & 3.088 \\
\hline 07/27/00 & 10:47:10 & 179.7362 & 9.102 & 29.905 & 3.094 \\
\hline 07/27/00 & 10:47:25 & 179.9862 & 9.097 & 29.905 & 3.099 \\
\hline 07/27/00 & 10:47:40 & 180.2362 & 9.094 & 29.905 & 3.102 \\
\hline 07/27/00 & 10:47:55 & 180.4862 & 9.091 & 29.907 & 3.105 \\
\hline 07/27/00 & 10:48:10 & 180.7362 & 9.088 & 29.905 & 3.108 \\
\hline 07/27/00 & $10: 48: 25$ & 180.9862 & 9.086 & 29.907 & 3.11 \\
\hline 07/27/00 & 10:48:40 & 181.2362 & 9.084 & 29.905 & 3.112 \\
\hline 07/27/00 & 10:48:55 & 181.4862 & 9.081 & 29.907 & 3.115 \\
\hline 07/27/00 & 10:49:10 & 181.7362 & 9.079 & 29.905 & 3.117 \\
\hline 07/27/00 & 10:49:25 & 181.9862 & 8.986 & 29.905 & 3.21 \\
\hline 07/27/00 & 10:49:40 & 182.2362 & 9.03 & 29.905 & 3.166 \\
\hline 07/27/00 & 10:49:55 & 182.4862 & 9.078 & 29.905 & 3.118 \\
\hline 07/27/00 & 10:50:10 & 182.7362 & 9.081 & 29.903 & 3.115 \\
\hline 07/27/00 & 10:50:25 & 182.9862 & 9.079 & 29.905 & 3.117 \\
\hline 07/27/00 & 10:50:40 & 183.2362 & 9.076 & 29.905 & 3.12 \\
\hline 07/27/00 & 10:50:55 & 183.4862 & 9.075 & 29.905 & 3.121 \\
\hline 07/27/00 & $10: 51: 10$ & 183.7362 & 9.072 & 29.903 & 3.124 \\
\hline 07/27/00 & $10: 51: 25$ & 183.9862 & 9.069 & 29.905 & 3.127 \\
\hline 07/27/00 & 10:51:40 & 184.2362 & 9.069 & 29.905 & 3.127 \\
\hline 07/27/00 & 10:51:55 & 184.4862 & 9.069 & 29.907 & 3.127 \\
\hline 07/27/00 & 10:52:10 & 184.7362 & 9.069 & 29.905 & 3.127 \\
\hline 07/27/00 & 10:52:25 & 184.9862 & 9.065 & 29.905 & 3.131 \\
\hline 07/27/00 & 10:52:40 & 185.2362 & 9.063 & 29.905 & 3.133 \\
\hline 07/27/00 & $10: 52: 55$ & 185.4862 & 9.062 & 29.905 & 3.134 \\
\hline 07/27/00 & 10:53:10 & 185.7362 & 9.063 & 29.905 & 3.133 \\
\hline 07/27/00 & 10:53:25 & 185.9862 & 9.061 & 29.905 & 3.135 \\
\hline 07/27/00 & 10:53:40 & 186.2362 & 8.955 & 29.905 & 3.241 \\
\hline 07/27/00 & 10:53:55 & 186.4862 & 8.965 & 29.905 & 3.231 \\
\hline
\end{tabular}




\section{Appendix B (continued)}

\begin{tabular}{|c|c|c|c|c|c|}
\hline $07 / 27 / 00$ & $10: 54: 10$ & 186.7362 & 9.035 & 29.905 & 3.161 \\
\hline $07 / 27 / 00$ & $10: 54: 25$ & 186.9862 & 9.076 & 29.905 & 3.12 \\
\hline $07 / 27 / 00$ & 10:54:40 & 187.2362 & 9.069 & 29.905 & 3.127 \\
\hline $07 / 27 / 00$ & 10:54:55 & 187.4862 & 9.065 & 29.905 & 3.131 \\
\hline $07 / 27 / 00$ & $10: 55: 10$ & 187.7362 & 9.063 & 29.907 & 3.133 \\
\hline $07 / 27 / 00$ & 10:55:25 & 187.9862 & 9.061 & 29.905 & 3.135 \\
\hline $07 / 27 / 00$ & 10:55:40 & 188.2362 & 9.058 & 29.905 & 3.138 \\
\hline $07 / 27 / 00$ & 10:55:55 & 188.4862 & 9.058 & 29.905 & 3.138 \\
\hline $07 / 27 / 00$ & 10:56:10 & 188.7362 & 9.056 & 29.905 & 3.14 \\
\hline $07 / 27 / 00$ & 10:56:25 & 188.9862 & 9.055 & 29.907 & 3.141 \\
\hline $07 / 27 / 00$ & $10: 56: 40$ & 189.2362 & 9.055 & 29.907 & 3.141 \\
\hline $07 / 27 / 00$ & 10:56:55 & 189.4862 & 9.055 & 29.905 & 3.141 \\
\hline $07 / 27 / 00$ & $10: 57: 10$ & 189.7362 & 9.056 & 29.907 & 3.14 \\
\hline 07/27/00 & 10:57:25 & 189.9862 & 8.96 & 29.907 & 3.236 \\
\hline $07 / 27 / 00$ & 10:57:40 & 190.2362 & 9.017 & 29.905 & 3.179 \\
\hline $07 / 27 / 00$ & 10:57:55 & 190.4862 & 9.056 & 29.907 & 3.14 \\
\hline $07 / 27 / 00$ & 10:58:10 & 190.7362 & 9.055 & 29.905 & 3.141 \\
\hline 07/27/00 & $10: 58: 25$ & 190.9862 & 9.053 & 29.905 & 3.143 \\
\hline $07 / 27 / 00$ & 10:58:40 & 191.2362 & 9.052 & 29.909 & 3.144 \\
\hline $07 / 27 / 00$ & 10:58:55 & 191.4862 & 9.05 & 29.905 & 3.146 \\
\hline $07 / 27 / 00$ & 10:59:10 & 191.7362 & 9.05 & 29.907 & 3.146 \\
\hline $07 / 27 / 00$ & 10:59:25 & 191.9862 & 9.048 & 29.907 & 3.148 \\
\hline $07 / 27 / 00$ & 10:59:40 & 192.2362 & 9.046 & 29.907 & 3.15 \\
\hline $07 / 27 / 00$ & 10:59:55 & 192.4862 & 9.046 & 29.905 & 3.15 \\
\hline $07 / 27 / 00$ & $11: 00: 10$ & 192.7362 & 9.045 & 29.905 & 3.151 \\
\hline $07 / 27 / 00$ & 11:00:25 & 192.9862 & 9.043 & 29.907 & 3.153 \\
\hline $07 / 27 / 00$ & 11:00:40 & 193.2362 & 9.043 & 29.907 & 3.153 \\
\hline $07 / 27 / 00$ & 11:00:55 & 193.4862 & 8.963 & 29.907 & 3.233 \\
\hline $07 / 27 / 00$ & 11:01:10 & 193.7362 & 9.006 & 29.905 & 3.19 \\
\hline $07 / 27 / 00$ & 11:01:25 & 193.9862 & 9.045 & 29.907 & 3.151 \\
\hline 07/27/00 & 11:01:40 & 194.2362 & 9.049 & 29.905 & 3.147 \\
\hline $07 / 27 / 00$ & 11:01:55 & 194.4862 & 9.046 & 29.905 & 3.15 \\
\hline $07 / 27 / 00$ & 11:02:10 & 194.7362 & 9.043 & 29.907 & 3.153 \\
\hline $07 / 27 / 00$ & 11:02:25 & 194.9862 & 9.043 & 29.905 & 3.153 \\
\hline $07 / 27 / 00$ & 11:02:40 & 195.2362 & 9.042 & 29.905 & 3.154 \\
\hline $07 / 27 / 00$ & 11:02:55 & 195.4862 & 9.042 & 29.907 & 3.154 \\
\hline 07/27/00 & 11:03:10 & 195.7362 & 9.04 & 29.905 & 3.156 \\
\hline $07 / 27 / 00$ & 11:03:25 & 195.9862 & 9.039 & 29.905 & 3.157 \\
\hline 07/27/00 & 11:03:40 & 196.2362 & 9.039 & 29.905 & 3.157 \\
\hline $07 / 27 / 00$ & 11:03:55 & 196.4862 & 9.039 & 29.905 & 3.157 \\
\hline $07 / 27 / 00$ & 11:04:10 & 196.7362 & 9.037 & 29.905 & 3.159 \\
\hline $07 / 27 / 00$ & 11:04:25 & 196.9862 & 9.037 & 29.905 & 3.159 \\
\hline 07/27/00 & 11:04:40 & 197.2362 & 9.037 & 29.905 & 3.159 \\
\hline 07/27/00 & 11:04:55 & 197.4862 & 9.037 & 29.905 & 3.159 \\
\hline $07 / 27 / 00$ & 11:05:10 & 197.7362 & 9.036 & 29.905 & 3.16 \\
\hline $07 / 27 / 00$ & 11:05:25 & 197.9862 & 8.955 & 29.905 & 3.241 \\
\hline $07 / 27 / 00$ & $11: 05: 40$ & 198.2362 & 8.947 & 29.903 & 3.249 \\
\hline $07 / 27 / 00$ & 11:05:55 & 198.4862 & 9.013 & 29.903 & 3.183 \\
\hline $07 / 27 / 00$ & 11:06:10 & 198.7362 & 9.037 & 29.905 & 3.159 \\
\hline $07 / 27 / 00$ & 11:06:25 & 198.9862 & 9.033 & 29.905 & 3.163 \\
\hline $07 / 27 / 00$ & 11:06:40 & 199.2362 & 9.033 & 29.903 & 3.163 \\
\hline
\end{tabular}




\section{Appendix B (continued)}

\begin{tabular}{|c|c|c|c|c|c|}
\hline $07 / 27 / 00$ & $11: 06: 55$ & 199.4862 & 9.032 & 29.905 & 3.164 \\
\hline 07/27/00 & 11:07:10 & 199.7362 & 9.032 & 29.905 & 3.164 \\
\hline 07/27/00 & 11:07:25 & 199.9862 & 9.03 & 29.903 & 3.166 \\
\hline 07/27/00 & 11:07:40 & 200.2362 & 9.03 & 29.905 & 3.166 \\
\hline 07/27/00 & 11:07:55 & 200.4862 & 9.03 & 29.905 & 3.166 \\
\hline 07/27/00 & 11:08:10 & 200.7362 & 9.027 & 29.905 & 3.169 \\
\hline 07/27/00 & 11:08:25 & 200.9862 & 9.003 & 29.903 & 3.193 \\
\hline 07/27/00 & 11:08:40 & 201.2362 & 8.925 & 29.905 & 3.271 \\
\hline 07/27/00 & 11:08:55 & 201.4862 & 8.983 & 29.905 & 3.213 \\
\hline 07/27/00 & 11:09:10 & 201.7362 & 9.037 & 29.903 & 3.159 \\
\hline 07/27/00 & $11: 09: 25$ & 201.9862 & 9.039 & 29.905 & 3.157 \\
\hline 07/27/00 & 11:09:40 & 202.2362 & 9.035 & 29.901 & 3.161 \\
\hline 07/27/00 & 11:09:55 & 202.4862 & 9.032 & 29.905 & 3.164 \\
\hline 07/27/00 & 11:10:10 & 202.7362 & 9.032 & 29.905 & 3.164 \\
\hline 07/27/00 & 11:10:25 & 202.9862 & 9.03 & 29.905 & 3.166 \\
\hline 07/27/00 & $11: 10: 40$ & 203.2362 & 9.03 & 29.903 & 3.166 \\
\hline 07/27/00 & 11:10:55 & 203.4862 & 9.03 & 29.903 & 3.166 \\
\hline 07/27/00 & 11:11:10 & 203.7362 & 9.016 & 29.903 & 3.18 \\
\hline 07/27/00 & 11:11:25 & 203.9862 & 8.948 & 29.903 & 3.248 \\
\hline 07/27/00 & 11:11:40 & 204.2362 & 9.009 & 29.903 & 3.187 \\
\hline 07/27/00 & 11:11:55 & 204.4862 & 9.016 & 29.903 & 3.18 \\
\hline 07/27/00 & 11:12:10 & 204.7362 & 9.016 & 29.903 & 3.18 \\
\hline 07/27/00 & $11: 12: 25$ & 204.9862 & 9.016 & 29.903 & 3.18 \\
\hline 07/27/00 & 11:12:40 & 205.2362 & 9.016 & 29.903 & 3.18 \\
\hline 07/27/00 & 11:12:55 & 205.4862 & 9.017 & 29.903 & 3.179 \\
\hline 07/27/00 & 11:13:10 & 205.7362 & 9.017 & 29.903 & 3.179 \\
\hline 07/27/00 & 11:13:25 & 205.9862 & 9.017 & 29.903 & 3.179 \\
\hline 07/27/00 & 11:13:40 & 206.2362 & 9.017 & 29.903 & 3.179 \\
\hline 07/27/00 & 11:13:55 & 206.4862 & 8.94 & 29.903 & 3.256 \\
\hline 07/27/00 & 11:14:10 & 206.7362 & 8.947 & 29.903 & 3.249 \\
\hline 07/27/00 & 11:14:25 & 206.9862 & 9.012 & 29.903 & 3.184 \\
\hline 07/27/00 & 11:14:40 & 207.2362 & 9.046 & 29.901 & 3.15 \\
\hline 07/27/00 & 11:14:55 & 207.4862 & 9.043 & 29.903 & 3.153 \\
\hline 07/27/00 & $11: 15: 10$ & 207.7362 & 9.039 & 29.903 & 3.157 \\
\hline 07/27/00 & 11:15:25 & 207.9862 & 9.035 & 29.903 & 3.161 \\
\hline 07/27/00 & 11:15:40 & 208.2362 & 9.03 & 29.903 & 3.166 \\
\hline 07/27/00 & 11:15:55 & 208.4862 & 9.026 & 29.903 & 3.17 \\
\hline 07/27/00 & 11:16:10 & 208.7362 & 9.025 & 29.903 & 3.171 \\
\hline 07/27/00 & 11:16:25 & 208.9862 & 9.023 & 29.905 & 3.173 \\
\hline 07/27/00 & 11:16:40 & 209.2362 & 8.965 & 29.903 & 3.231 \\
\hline 07/27/00 & 11:16:55 & 209.4862 & 8.963 & 29.903 & 3.233 \\
\hline 07/27/00 & 11:17:10 & 209.7362 & 9.014 & 29.903 & 3.182 \\
\hline 07/27/00 & 11:17:25 & 209.9862 & 9.03 & 29.903 & 3.166 \\
\hline 07/27/00 & 11:17:40 & 210.2362 & 9.025 & 29.903 & 3.171 \\
\hline 07/27/00 & 11:17:55 & 210.4862 & 9.022 & 29.903 & 3.174 \\
\hline 07/27/00 & $11: 18: 10$ & 210.7362 & 9.019 & 29.903 & 3.177 \\
\hline 07/27/00 & 11:18:25 & 210.9862 & 9.019 & 29.903 & 3.177 \\
\hline 07/27/00 & 11:18:40 & 211.2362 & 9.017 & 29.903 & 3.179 \\
\hline 07/27/00 & 11:18:55 & 211.4862 & 9.016 & 29.901 & 3.18 \\
\hline 07/27/00 & 11:19:10 & 211.7362 & 9.014 & 29.903 & 3.182 \\
\hline 07/27/00 & 11:19:25 & 211.9862 & 9.013 & 29.901 & 3.183 \\
\hline
\end{tabular}




\section{Appendix B (continued)}

\begin{tabular}{|c|c|c|c|c|c|}
\hline $07 / 27 / 00$ & $11: 19: 40$ & 212.2362 & 8.925 & 29.903 & 3.271 \\
\hline $07 / 27 / 00$ & 11:19:55 & 212.4862 & 8.909 & 29.903 & 3.287 \\
\hline $07 / 27 / 00$ & 11:20:10 & 212.7362 & 8.983 & 29.901 & 3.213 \\
\hline $07 / 27 / 00$ & 11:20:25 & 212.9862 & 9.02 & 29.903 & 3.176 \\
\hline $07 / 27 / 00$ & 11:20:40 & 213.2362 & 9.017 & 29.901 & 3.179 \\
\hline $07 / 27 / 00$ & 11:20:55 & 213.4862 & 9.014 & 29.901 & 3.182 \\
\hline $07 / 27 / 00$ & 11:21:10 & 213.7362 & 9.013 & 29.901 & 3.183 \\
\hline $07 / 27 / 00$ & 11:21:25 & 213.9862 & 9.012 & 29.901 & 3.184 \\
\hline $07 / 27 / 00$ & 11:21:40 & 214.2362 & 9.01 & 29.901 & 3.186 \\
\hline $07 / 27 / 00$ & 11:21:55 & 214.4862 & 9.01 & 29.901 & 3.186 \\
\hline $07 / 27 / 00$ & $11: 22: 10$ & 214.7362 & 9.009 & 29.901 & 3.187 \\
\hline $07 / 27 / 00$ & 11:22:25 & 214.9862 & 9.006 & 29.903 & 3.19 \\
\hline $07 / 27 / 00$ & 11:22:40 & 215.2362 & 8.968 & 29.901 & 3.228 \\
\hline 07/27/00 & 11:22:55 & 215.4862 & 8.947 & 29.901 & 3.249 \\
\hline $07 / 27 / 00$ & 11:23:10 & 215.7362 & 9 & 29.901 & 3.196 \\
\hline $07 / 27 / 00$ & 11:23:25 & 215.9862 & 9.003 & 29.901 & 3.193 \\
\hline $07 / 27 / 00$ & 11:23:40 & 216.2362 & 9.003 & 29.903 & 3.193 \\
\hline $07 / 27 / 00$ & 11:23:55 & 216.4862 & 9.001 & 29.901 & 3.195 \\
\hline $07 / 27 / 00$ & $11: 24: 10$ & 216.7362 & 9.001 & 29.901 & 3.195 \\
\hline $07 / 27 / 00$ & 11:24:25 & 216.9862 & 9.001 & 29.901 & 3.195 \\
\hline $07 / 27 / 00$ & 11:24:40 & 217.2362 & 9 & 29.901 & 3.196 \\
\hline $07 / 27 / 00$ & 11:24:55 & 217.4862 & 9 & 29.901 & 3.196 \\
\hline $07 / 27 / 00$ & $11: 25: 10$ & 217.7362 & 9 & 29.901 & 3.196 \\
\hline $07 / 27 / 00$ & $11: 25: 25$ & 217.9862 & 8.999 & 29.901 & 3.197 \\
\hline 07/27/00 & 11:25:40 & 218.2362 & 8.999 & 29.901 & 3.197 \\
\hline $07 / 27 / 00$ & 11:25:55 & 218.4862 & 8.917 & 29.903 & 3.279 \\
\hline $07 / 27 / 00$ & $11: 26: 10$ & 218.7362 & 8.961 & 29.903 & 3.235 \\
\hline $07 / 27 / 00$ & $11: 26: 25$ & 218.9862 & 9.001 & 29.903 & 3.195 \\
\hline $07 / 27 / 00$ & $11: 26: 40$ & 219.2362 & 8.999 & 29.901 & 3.197 \\
\hline $07 / 27 / 00$ & $11: 26: 55$ & 219.4862 & 8.996 & 29.901 & 3.2 \\
\hline $07 / 27 / 00$ & 11:27:10 & 219.7362 & 8.994 & 29.903 & 3.202 \\
\hline $07 / 27 / 00$ & 11:27:25 & 219.9862 & 8.993 & 29.903 & 3.203 \\
\hline $07 / 27 / 00$ & 11:27:40 & 220.2362 & 8.993 & 29.903 & 3.203 \\
\hline $07 / 27 / 00$ & 11:27:55 & 220.4862 & 8.991 & 29.901 & 3.205 \\
\hline $07 / 27 / 00$ & $11: 28: 10$ & 220.7362 & 8.991 & 29.901 & 3.205 \\
\hline $07 / 27 / 00$ & 11:28:25 & 220.9862 & 8.99 & 29.901 & 3.206 \\
\hline 07/27/00 & 11:28:40 & 221.2362 & 8.906 & 29.901 & 3.29 \\
\hline $07 / 27 / 00$ & 11:28:55 & 221.4862 & 8.961 & 29.903 & 3.235 \\
\hline 07/27/00 & 11:29:10 & 221.7362 & 8.996 & 29.901 & 3.2 \\
\hline $07 / 27 / 00$ & 11:29:25 & 221.9862 & 8.994 & 29.901 & 3.202 \\
\hline $07 / 27 / 00$ & 11:29:40 & 222.2362 & 8.991 & 29.901 & 3.205 \\
\hline $07 / 27 / 00$ & 11:29:55 & 222.4862 & 8.99 & 29.901 & 3.206 \\
\hline 07/27/00 & 11:30:10 & 222.7362 & 8.989 & 29.901 & 3.207 \\
\hline 07/27/00 & 11:30:25 & 222.9862 & 8.989 & 29.901 & 3.207 \\
\hline $07 / 27 / 00$ & 11:30:40 & 223.2362 & 8.987 & 29.901 & 3.209 \\
\hline $07 / 27 / 00$ & 11:30:55 & 223.4862 & 8.971 & 29.899 & 3.225 \\
\hline $07 / 27 / 00$ & 11:31:10 & 223.7362 & 8.911 & 29.901 & 3.285 \\
\hline $07 / 27 / 00$ & $11: 31: 25$ & 223.9862 & 8.968 & 29.901 & 3.228 \\
\hline $07 / 27 / 00$ & 11:31:40 & 224.2362 & 8.978 & 29.901 & 3.218 \\
\hline $07 / 27 / 00$ & 11:31:55 & 224.4862 & 8.978 & 29.901 & 3.218 \\
\hline $07 / 27 / 00$ & 11:32:10 & 224.7362 & 8.978 & 29.901 & 3.218 \\
\hline
\end{tabular}




\section{Appendix B (continued)}

\begin{tabular}{|c|c|c|c|c|c|}
\hline $07 / 27 / 00$ & $11: 32: 25$ & 224.9862 & 8.978 & 29.899 & 3.218 \\
\hline 07/27/00 & $11: 32: 40$ & 225.2362 & 8.978 & 29.901 & 3.218 \\
\hline 07/27/00 & 11:32:55 & 225.4862 & 8.978 & 29.901 & 3.218 \\
\hline 07/27/00 & $11: 33: 10$ & 225.7362 & 8.977 & 29.903 & 3.219 \\
\hline 07/27/00 & $11: 33: 25$ & 225.9862 & 8.978 & 29.901 & 3.218 \\
\hline 07/27/00 & $11: 33: 40$ & 226.2362 & 8.977 & 29.901 & 3.219 \\
\hline 07/27/00 & 11:33:55 & 226.4862 & 8.977 & 29.901 & 3.219 \\
\hline 07/27/00 & 11:34:10 & 226.7362 & 8.977 & 29.899 & 3.219 \\
\hline 07/27/00 & $11: 34: 25$ & 226.9862 & 8.977 & 29.903 & 3.219 \\
\hline 07/27/00 & $11: 34: 40$ & 227.2362 & 8.934 & 29.901 & 3.262 \\
\hline 07/27/00 & $11: 34: 55$ & 227.4862 & 8.921 & 29.901 & 3.275 \\
\hline 07/27/00 & 11:35:10 & 227.7362 & 8.925 & 29.903 & 3.271 \\
\hline 07/27/00 & 11:35:25 & 227.9862 & 8.947 & 29.903 & 3.249 \\
\hline 07/27/00 & 11:35:40 & 228.2362 & 8.98 & 29.901 & 3.216 \\
\hline 07/27/00 & 11:35:55 & 228.4862 & 9.004 & 29.901 & 3.192 \\
\hline 07/27/00 & $11: 36: 10$ & 228.7362 & 9 & 29.903 & 3.196 \\
\hline 07/27/00 & $11: 36: 25$ & 228.9862 & 8.996 & 29.901 & 3.2 \\
\hline 07/27/00 & $11: 36: 40$ & 229.2362 & 8.993 & 29.901 & 3.203 \\
\hline 07/27/00 & 11:36:55 & 229.4862 & 8.989 & 29.901 & 3.207 \\
\hline 07/27/00 & 11:37:10 & 229.7362 & 8.987 & 29.901 & 3.209 \\
\hline 07/27/00 & 11:37:25 & 229.9862 & 8.986 & 29.901 & 3.21 \\
\hline 07/27/00 & $11: 37: 40$ & 230.2362 & 8.984 & 29.901 & 3.212 \\
\hline 07/27/00 & $11: 37: 55$ & 230.4862 & 8.981 & 29.901 & 3.215 \\
\hline 07/27/00 & $11: 38: 10$ & 230.7362 & 8.98 & 29.901 & 3.216 \\
\hline 07/27/00 & 11:38:25 & 230.9862 & 8.974 & 29.901 & 3.222 \\
\hline 07/27/00 & $11: 38: 40$ & 231.2362 & 8.918 & 29.901 & 3.278 \\
\hline 07/27/00 & 11:38:55 & 231.4862 & 8.964 & 29.899 & 3.232 \\
\hline 07/27/00 & 11:39:10 & 231.7362 & 8.993 & 29.901 & 3.203 \\
\hline 07/27/00 & $11: 39: 25$ & 231.9862 & 8.989 & 29.899 & 3.207 \\
\hline 07/27/00 & 11:39:40 & 232.2362 & 8.987 & 29.901 & 3.209 \\
\hline 07/27/00 & 11:39:55 & 232.4862 & 8.981 & 29.899 & 3.215 \\
\hline 07/27/00 & 11:40:10 & 232.7362 & 8.981 & 29.899 & 3.215 \\
\hline 07/27/00 & 11:40:25 & 232.9862 & 8.98 & 29.901 & 3.216 \\
\hline 07/27/00 & 11:40:40 & 233.2362 & 8.978 & 29.899 & 3.218 \\
\hline 07/27/00 & 11:40:55 & 233.4862 & 8.976 & 29.899 & 3.22 \\
\hline 07/27/00 & 11:41:10 & 233.7362 & 8.976 & 29.899 & 3.22 \\
\hline 07/27/00 & 11:41:25 & 233.9862 & 8.974 & 29.899 & 3.222 \\
\hline 07/27/00 & 11:41:40 & 234.2362 & 8.973 & 29.899 & 3.223 \\
\hline 07/27/00 & 11:41:55 & 234.4862 & 8.973 & 29.899 & 3.223 \\
\hline 07/27/00 & 11:42:10 & 234.7362 & 8.974 & 29.899 & 3.222 \\
\hline 07/27/00 & 11:42:25 & 234.9862 & 8.973 & 29.899 & 3.223 \\
\hline 07/27/00 & 11:42:40 & 235.2362 & 8.957 & 29.899 & 3.239 \\
\hline 07/27/00 & 11:42:55 & 235.4862 & 8.891 & 29.899 & 3.305 \\
\hline 07/27/00 & 11:43:10 & 235.7362 & 8.958 & 29.897 & 3.238 \\
\hline 07/27/00 & 11:43:25 & 235.9862 & 8.98 & 29.899 & 3.216 \\
\hline 07/27/00 & 11:43:40 & 236.2362 & 8.977 & 29.899 & 3.219 \\
\hline 07/27/00 & 11:43:55 & 236.4862 & 8.976 & 29.899 & 3.22 \\
\hline 07/27/00 & 11:44:10 & 236.7362 & 8.974 & 29.899 & 3.222 \\
\hline 07/27/00 & 11:44:25 & 236.9862 & 8.973 & 29.899 & 3.223 \\
\hline 07/27/00 & 11:44:40 & 237.2362 & 8.971 & 29.899 & 3.225 \\
\hline 07/27/00 & 11:44:55 & 237.4862 & 8.971 & 29.899 & 3.225 \\
\hline
\end{tabular}




\section{Appendix B (continued)}

\begin{tabular}{|c|c|c|c|c|c|}
\hline $07 / 27 / 00$ & $11: 45: 10$ & 237.7362 & 8.971 & 29.899 & 3.225 \\
\hline $07 / 27 / 00$ & $11: 45: 25$ & 237.9862 & 8.971 & 29.899 & 3.225 \\
\hline $07 / 27 / 00$ & $11: 45: 40$ & 238.2362 & 8.971 & 29.899 & 3.225 \\
\hline $07 / 27 / 00$ & $11: 45: 55$ & 238.4862 & 8.954 & 29.901 & 3.242 \\
\hline $07 / 27 / 00$ & $11: 46: 10$ & 238.7362 & 8.932 & 29.901 & 3.264 \\
\hline $07 / 27 / 00$ & $11: 46: 25$ & 238.9862 & 8.944 & 29.899 & 3.252 \\
\hline $07 / 27 / 00$ & 11:46:40 & 239.2362 & 8.994 & 29.899 & 3.202 \\
\hline $07 / 27 / 00$ & $11: 46: 55$ & 239.4862 & 8.997 & 29.899 & 3.199 \\
\hline $07 / 27 / 00$ & $11: 47: 10$ & 239.7362 & 8.99 & 29.899 & 3.206 \\
\hline $07 / 27 / 00$ & $11: 47: 25$ & 239.9862 & 8.986 & 29.899 & 3.21 \\
\hline $07 / 27 / 00$ & $11: 47: 40$ & 240.2362 & 8.983 & 29.899 & 3.213 \\
\hline $07 / 27 / 00$ & 11:47:55 & 240.4862 & 8.978 & 29.899 & 3.218 \\
\hline $07 / 27 / 00$ & 11:48:10 & 240.7362 & 8.977 & 29.897 & 3.219 \\
\hline $07 / 27 / 00$ & 11:48:25 & 240.9862 & 8.976 & 29.897 & 3.22 \\
\hline $07 / 27 / 00$ & 11:48:40 & 241.2362 & 8.974 & 29.897 & 3.222 \\
\hline $07 / 27 / 00$ & $11: 48: 55$ & 241.4862 & 8.97 & 29.897 & 3.226 \\
\hline $07 / 27 / 00$ & 11:49:10 & 241.7362 & 8.921 & 29.899 & 3.275 \\
\hline $07 / 27 / 00$ & 11:49:25 & 241.9862 & 8.967 & 29.897 & 3.229 \\
\hline $07 / 27 / 00$ & 11:49:40 & 242.2362 & 8.968 & 29.899 & 3.228 \\
\hline $07 / 27 / 00$ & $11: 49: 55$ & 242.4862 & 8.967 & 29.897 & 3.229 \\
\hline $07 / 27 / 00$ & $11: 50: 10$ & 242.7362 & 8.965 & 29.895 & 3.231 \\
\hline $07 / 27 / 00$ & $11: 50: 25$ & 242.9862 & 8.964 & 29.899 & 3.232 \\
\hline $07 / 27 / 00$ & 11:50:40 & 243.2362 & 8.964 & 29.897 & 3.232 \\
\hline $07 / 27 / 00$ & $11: 50: 55$ & 243.4862 & 8.963 & 29.897 & 3.233 \\
\hline $07 / 27 / 00$ & $11: 51: 10$ & 243.7362 & 8.963 & 29.897 & 3.233 \\
\hline $07 / 27 / 00$ & $11: 51: 25$ & 243.9862 & 8.963 & 29.897 & 3.233 \\
\hline $07 / 27 / 00$ & $11: 51: 40$ & 244.2362 & 8.963 & 29.895 & 3.233 \\
\hline $07 / 27 / 00$ & $11: 51: 55$ & 244.4862 & 8.963 & 29.899 & 3.233 \\
\hline $07 / 27 / 00$ & 11:52:10 & 244.7362 & 8.964 & 29.897 & 3.232 \\
\hline $07 / 27 / 00$ & 11:52:25 & 244.9862 & 8.963 & 29.897 & 3.233 \\
\hline $07 / 27 / 00$ & 11:52:40 & 245.2362 & 8.963 & 29.899 & 3.233 \\
\hline $07 / 27 / 00$ & 11:52:55 & 245.4862 & 8.963 & 29.897 & 3.233 \\
\hline $07 / 27 / 00$ & 11:53:10 & 245.7362 & 8.961 & 29.897 & 3.235 \\
\hline $07 / 27 / 00$ & 11:53:25 & 245.9862 & 8.895 & 29.895 & 3.301 \\
\hline $07 / 27 / 00$ & 11:53:40 & 246.2362 & 8.934 & 29.899 & 3.262 \\
\hline $07 / 27 / 00$ & 11:53:55 & 246.4862 & 8.964 & 29.897 & 3.232 \\
\hline $07 / 27 / 00$ & $11: 54: 10$ & 246.7362 & 8.914 & 29.895 & 3.282 \\
\hline $07 / 27 / 00$ & $11: 54: 25$ & 246.9862 & 8.883 & 29.897 & 3.313 \\
\hline $07 / 27 / 00$ & $11: 54: 40$ & 247.2362 & 8.945 & 29.897 & 3.251 \\
\hline $07 / 27 / 00$ & $11: 54: 55$ & 247.4862 & 8.97 & 29.895 & 3.226 \\
\hline $07 / 27 / 00$ & 11:55:10 & 247.7362 & 8.872 & 29.895 & 3.324 \\
\hline $07 / 27 / 00$ & 11:55:25 & 247.9862 & 8.917 & 29.899 & 3.279 \\
\hline $07 / 27 / 00$ & $11: 55: 40$ & 248.2362 & 8.971 & 29.897 & 3.225 \\
\hline $07 / 27 / 00$ & $11: 55: 55$ & 248.4862 & 8.994 & 29.897 & 3.202 \\
\hline $07 / 27 / 00$ & $11: 56: 10$ & 248.7362 & 8.99 & 29.897 & 3.206 \\
\hline $07 / 27 / 00$ & $11: 56: 25$ & 248.9862 & 8.984 & 29.897 & 3.212 \\
\hline $07 / 27 / 00$ & $11: 56: 40$ & 249.2362 & 8.978 & 29.897 & 3.218 \\
\hline $07 / 27 / 00$ & 11:56:55 & 249.4862 & 8.976 & 29.897 & 3.22 \\
\hline $07 / 27 / 00$ & $11: 57: 10$ & 249.7362 & 8.971 & 29.895 & 3.225 \\
\hline $07 / 27 / 00$ & 11:57:25 & 249.9862 & 8.968 & 29.897 & 3.228 \\
\hline $07 / 27 / 00$ & $11: 57: 40$ & 250.2362 & 8.967 & 29.897 & 3.229 \\
\hline
\end{tabular}




\section{Appendix B (continued)}

\begin{tabular}{|c|c|c|c|c|c|}
\hline $07 / 27 / 00$ & $11: 57: 55$ & 250.4862 & 8.965 & 29.897 & 3.231 \\
\hline 07/27/00 & 11:58:10 & 250.7362 & 8.963 & 29.895 & 3.233 \\
\hline 07/27/00 & 11:58:25 & 250.9862 & 8.961 & 29.897 & 3.235 \\
\hline 07/27/00 & 11:58:40 & 251.2362 & 8.96 & 29.895 & 3.236 \\
\hline 07/27/00 & 11:58:55 & 251.4862 & 8.958 & 29.897 & 3.238 \\
\hline 07/27/00 & 11:59:10 & 251.7362 & 8.958 & 29.895 & 3.238 \\
\hline 07/27/00 & 11:59:25 & 251.9862 & 8.958 & 29.895 & 3.238 \\
\hline 07/27/00 & 11:59:40 & 252.2362 & 8.909 & 29.895 & 3.287 \\
\hline 07/27/00 & 11:59:55 & 252.4862 & 8.918 & 29.897 & 3.278 \\
\hline 07/27/00 & 12:00:10 & 252.7362 & 8.948 & 29.897 & 3.248 \\
\hline 07/27/00 & 12:00:25 & 252.9862 & 8.948 & 29.895 & 3.248 \\
\hline 07/27/00 & 12:00:40 & 253.2362 & 8.948 & 29.895 & 3.248 \\
\hline 07/27/00 & 12:00:55 & 253.4862 & 8.935 & 29.895 & 3.261 \\
\hline 07/27/00 & 12:01:10 & 253.7362 & 8.948 & 29.895 & 3.248 \\
\hline 07/27/00 & 12:01:25 & 253.9862 & 8.948 & 29.895 & 3.248 \\
\hline 07/27/00 & 12:01:40 & 254.2362 & 8.947 & 29.897 & 3.249 \\
\hline 07/27/00 & 12:01:55 & 254.4862 & 8.947 & 29.895 & 3.249 \\
\hline 07/27/00 & 12:02:10 & 254.7362 & 8.922 & 29.895 & 3.274 \\
\hline 07/27/00 & 12:02:25 & 254.9862 & 8.882 & 29.893 & 3.314 \\
\hline 07/27/00 & 12:02:40 & 255.2362 & 8.945 & 29.895 & 3.251 \\
\hline 07/27/00 & 12:02:55 & 255.4862 & 8.973 & 29.895 & 3.223 \\
\hline 07/27/00 & 12:03:10 & 255.7362 & 8.965 & 29.893 & 3.231 \\
\hline 07/27/00 & 12:03:25 & 255.9862 & 8.967 & 29.895 & 3.229 \\
\hline 07/27/00 & 12:03:40 & 256.2362 & 8.964 & 29.895 & 3.232 \\
\hline 07/27/00 & 12:03:55 & 256.4862 & 8.963 & 29.895 & 3.233 \\
\hline 07/27/00 & 12:04:10 & 256.7362 & 8.961 & 29.893 & 3.235 \\
\hline 07/27/00 & 12:04:25 & 256.9862 & 8.96 & 29.893 & 3.236 \\
\hline 07/27/00 & 12:04:40 & 257.2362 & 8.958 & 29.893 & 3.238 \\
\hline 07/27/00 & 12:04:55 & 257.4862 & 8.957 & 29.893 & 3.239 \\
\hline 07/27/00 & 12:05:10 & 257.7362 & 8.922 & 29.893 & 3.274 \\
\hline 07/27/00 & 12:05:25 & 257.9862 & 8.901 & 29.893 & 3.295 \\
\hline 07/27/00 & 12:05:40 & 258.2362 & 8.917 & 29.891 & 3.279 \\
\hline 07/27/00 & 12:05:55 & 258.4862 & 8.906 & 29.893 & 3.29 \\
\hline 07/27/00 & $12: 06: 10$ & 258.7362 & 8.888 & 29.893 & 3.308 \\
\hline 07/27/00 & 12:06:25 & 258.9862 & 8.876 & 29.893 & 3.32 \\
\hline 07/27/00 & 12:06:40 & 259.2362 & 8.865 & 29.893 & 3.331 \\
\hline 07/27/00 & 12:06:55 & 259.4862 & 8.855 & 29.891 & 3.341 \\
\hline 07/27/00 & 12:07:10 & 259.7362 & 8.845 & 29.893 & 3.351 \\
\hline 07/27/00 & 12:07:25 & 259.9862 & 8.837 & 29.893 & 3.359 \\
\hline 07/27/00 & 12:07:40 & 260.2362 & 8.829 & 29.893 & 3.367 \\
\hline 07/27/00 & 12:07:55 & 260.4862 & 8.824 & 29.893 & 3.372 \\
\hline 07/27/00 & 12:08:10 & 260.7362 & 8.819 & 29.893 & 3.377 \\
\hline 07/27/00 & 12:08:25 & 260.9862 & 8.814 & 29.893 & 3.382 \\
\hline 07/27/00 & 12:08:40 & 261.2362 & 8.81 & 29.893 & 3.386 \\
\hline 07/27/00 & 12:08:55 & 261.4862 & 8.806 & 29.895 & 3.39 \\
\hline 07/27/00 & 12:09:10 & 261.7362 & 8.801 & 29.895 & 3.395 \\
\hline 07/27/00 & 12:09:25 & 261.9862 & 8.8 & 29.895 & 3.396 \\
\hline 07/27/00 & 12:09:40 & 262.2362 & 8.797 & 29.895 & 3.399 \\
\hline 07/27/00 & 12:09:55 & 262.4862 & 8.796 & 29.893 & 3.4 \\
\hline 07/27/00 & 12:10:10 & 262.7362 & 8.794 & 29.895 & 3.402 \\
\hline 07/27/00 & 12:10:25 & 262.9862 & 8.791 & 29.893 & 3.405 \\
\hline
\end{tabular}




\section{Appendix B (continued)}

\begin{tabular}{|c|c|c|c|c|c|}
\hline $07 / 27 / 00$ & $12: 10: 40$ & 263.2362 & 8.79 & 29.893 & 3.406 \\
\hline $07 / 27 / 00$ & 12:10:55 & 263.4862 & 8.788 & 29.893 & 3.408 \\
\hline $07 / 27 / 00$ & 12:11:10 & 263.7362 & 8.788 & 29.893 & 3.408 \\
\hline $07 / 27 / 00$ & $12: 11: 25$ & 263.9862 & 8.787 & 29.895 & 3.409 \\
\hline $07 / 27 / 00$ & $12: 11: 40$ & 264.2362 & 8.787 & 29.895 & 3.409 \\
\hline $07 / 27 / 00$ & 12:11:55 & 264.4862 & 8.785 & 29.895 & 3.411 \\
\hline $07 / 27 / 00$ & 12:12:10 & 264.7362 & 8.783 & 29.893 & 3.413 \\
\hline $07 / 27 / 00$ & 12:12:25 & 264.9862 & 8.752 & 29.893 & 3.444 \\
\hline $07 / 27 / 00$ & 12:12:40 & 265.2362 & 8.751 & 29.893 & 3.445 \\
\hline $07 / 27 / 00$ & 12:12:55 & 265.4862 & 8.796 & 29.893 & 3.4 \\
\hline $07 / 27 / 00$ & 12:13:10 & 265.7362 & 8.814 & 29.893 & 3.382 \\
\hline $07 / 27 / 00$ & 12:13:25 & 265.9862 & 8.813 & 29.891 & 3.383 \\
\hline $07 / 27 / 00$ & 12:13:40 & 266.2362 & 8.804 & 29.893 & 3.392 \\
\hline $07 / 27 / 00$ & 12:13:55 & 266.4862 & 8.798 & 29.891 & 3.398 \\
\hline $07 / 27 / 00$ & 12:14:10 & 266.7362 & 8.794 & 29.891 & 3.402 \\
\hline $07 / 27 / 00$ & 12:14:25 & 266.9862 & 8.79 & 29.891 & 3.406 \\
\hline $07 / 27 / 00$ & 12:14:40 & 267.2362 & 8.785 & 29.893 & 3.411 \\
\hline $07 / 27 / 00$ & 12:14:55 & 267.4862 & 8.784 & 29.893 & 3.412 \\
\hline $07 / 27 / 00$ & $12: 15: 10$ & 267.7362 & 8.781 & 29.893 & 3.415 \\
\hline $07 / 27 / 00$ & $12: 15: 25$ & 267.9862 & 8.778 & 29.891 & 3.418 \\
\hline $07 / 27 / 00$ & $12: 15: 40$ & 268.2362 & 8.775 & 29.893 & 3.421 \\
\hline $07 / 27 / 00$ & $12: 15: 55$ & 268.4862 & 8.775 & 29.891 & 3.421 \\
\hline $07 / 27 / 00$ & 12:16:10 & 268.7362 & 8.773 & 29.891 & 3.423 \\
\hline $07 / 27 / 00$ & 12:16:25 & 268.9862 & 8.771 & 29.891 & 3.425 \\
\hline $07 / 27 / 00$ & $12: 16: 40$ & 269.2362 & 8.767 & 29.891 & 3.429 \\
\hline $07 / 27 / 00$ & $12: 16: 55$ & 269.4862 & 8.77 & 29.888 & 3.426 \\
\hline $07 / 27 / 00$ & 12:17:10 & 269.7362 & 8.765 & 29.891 & 3.431 \\
\hline $07 / 27 / 00$ & $12: 17: 25$ & 269.9862 & 8.765 & 29.891 & 3.431 \\
\hline $07 / 27 / 00$ & $12: 17: 40$ & 270.2362 & 8.764 & 29.891 & 3.432 \\
\hline $07 / 27 / 00$ & $12: 17: 55$ & 270.4862 & 8.764 & 29.891 & 3.432 \\
\hline $07 / 27 / 00$ & 12:18:10 & 270.7362 & 8.764 & 29.891 & 3.432 \\
\hline $07 / 27 / 00$ & 12:18:25 & 270.9862 & 8.762 & 29.891 & 3.434 \\
\hline $07 / 27 / 00$ & $12: 18: 40$ & 271.2362 & 8.762 & 29.891 & 3.434 \\
\hline $07 / 27 / 00$ & $12: 18: 55$ & 271.4862 & 8.762 & 29.888 & 3.434 \\
\hline $07 / 27 / 00$ & 12:19:10 & 271.7362 & 8.721 & 29.891 & 3.475 \\
\hline $07 / 27 / 00$ & 12:19:25 & 271.9862 & 8.715 & 29.891 & 3.481 \\
\hline $07 / 27 / 00$ & 12:19:40 & 272.2362 & 8.737 & 29.891 & 3.459 \\
\hline $07 / 27 / 00$ & 12:19:55 & 272.4862 & 8.809 & 29.891 & 3.387 \\
\hline $07 / 27 / 00$ & $12: 20: 10$ & 272.7362 & 8.875 & 29.888 & 3.321 \\
\hline $07 / 27 / 00$ & $12: 20: 25$ & 272.9862 & 8.872 & 29.891 & 3.324 \\
\hline $07 / 27 / 00$ & 12:20:40 & 273.2362 & 8.901 & 29.888 & 3.295 \\
\hline $07 / 27 / 00$ & 12:20:55 & 273.4862 & 8.902 & 29.891 & 3.294 \\
\hline $07 / 27 / 00$ & $12: 21: 10$ & 273.7362 & 8.904 & 29.888 & 3.292 \\
\hline $07 / 27 / 00$ & $12: 21: 25$ & 273.9862 & 8.904 & 29.888 & 3.292 \\
\hline $07 / 27 / 00$ & $12: 21: 40$ & 274.2362 & 8.904 & 29.891 & 3.292 \\
\hline $07 / 27 / 00$ & $12: 21: 55$ & 274.4862 & 8.905 & 29.891 & 3.291 \\
\hline $07 / 27 / 00$ & 12:22:10 & 274.7362 & 8.905 & 29.888 & 3.291 \\
\hline $07 / 27 / 00$ & 12:22:25 & 274.9862 & 8.905 & 29.888 & 3.291 \\
\hline $07 / 27 / 00$ & 12:22:40 & 275.2362 & 8.904 & 29.888 & 3.292 \\
\hline $07 / 27 / 00$ & 12:22:55 & 275.4862 & 8.918 & 29.891 & 3.278 \\
\hline $07 / 27 / 00$ & 12:23:10 & 275.7362 & 8.915 & 29.891 & 3.281 \\
\hline
\end{tabular}




\section{Appendix B (continued)}

\begin{tabular}{|c|c|c|c|c|c|}
\hline $07 / 27 / 00$ & $12: 23: 25$ & 275.9862 & 8.914 & 29.891 & 3.282 \\
\hline 07/27/00 & 12:23:40 & 276.2362 & 8.912 & 29.888 & 3.284 \\
\hline 07/27/00 & 12:23:55 & 276.4862 & 8.912 & 29.888 & 3.284 \\
\hline 07/27/00 & $12: 24: 10$ & 276.7362 & 8.912 & 29.888 & 3.284 \\
\hline 07/27/00 & 12:24:25 & 276.9862 & 8.911 & 29.888 & 3.285 \\
\hline 07/27/00 & 12:24:40 & 277.2362 & 8.919 & 29.888 & 3.277 \\
\hline 07/27/00 & 12:24:55 & 277.4862 & 8.918 & 29.888 & 3.278 \\
\hline 07/27/00 & 12:25:10 & 277.7362 & 8.915 & 29.888 & 3.281 \\
\hline 07/27/00 & $12: 25: 25$ & 277.9862 & 8.915 & 29.888 & 3.281 \\
\hline 07/27/00 & $12: 25: 40$ & 278.2362 & 8.914 & 29.886 & 3.282 \\
\hline 07/27/00 & 12:25:55 & 278.4862 & 8.924 & 29.886 & 3.272 \\
\hline 07/27/00 & 12:26:10 & 278.7362 & 8.921 & 29.886 & 3.275 \\
\hline 07/27/00 & 12:26:25 & 278.9862 & 8.918 & 29.888 & 3.278 \\
\hline 07/27/00 & 12:26:40 & 279.2362 & 8.917 & 29.886 & 3.279 \\
\hline 07/27/00 & 12:26:55 & 279.4862 & 8.921 & 29.886 & 3.275 \\
\hline 07/27/00 & $12: 27: 10$ & 279.7362 & 8.924 & 29.886 & 3.272 \\
\hline 07/27/00 & 12:27:25 & 279.9862 & 8.921 & 29.884 & 3.275 \\
\hline 07/27/00 & 12:27:40 & 280.2362 & 8.918 & 29.886 & 3.278 \\
\hline 07/27/00 & 12:27:55 & 280.4862 & 8.917 & 29.884 & 3.279 \\
\hline 07/27/00 & 12:28:10 & 280.7362 & 8.922 & 29.884 & 3.274 \\
\hline 07/27/00 & 12:28:25 & 280.9862 & 8.927 & 29.886 & 3.269 \\
\hline 07/27/00 & $12: 28: 40$ & 281.2362 & 8.924 & 29.884 & 3.272 \\
\hline 07/27/00 & 12:28:55 & 281.4862 & 8.922 & 29.884 & 3.274 \\
\hline 07/27/00 & 12:29:10 & 281.7362 & 8.929 & 29.882 & 3.267 \\
\hline 07/27/00 & 12:29:25 & 281.9862 & 8.928 & 29.882 & 3.268 \\
\hline 07/27/00 & 12:29:40 & 282.2362 & 8.925 & 29.882 & 3.271 \\
\hline 07/27/00 & 12:29:55 & 282.4862 & 8.929 & 29.882 & 3.267 \\
\hline 07/27/00 & 12:30:10 & 282.7362 & 8.934 & 29.882 & 3.262 \\
\hline 07/27/00 & 12:30:25 & 282.9862 & 8.932 & 29.882 & 3.264 \\
\hline 07/27/00 & 12:30:40 & 283.2362 & 8.863 & 29.882 & 3.333 \\
\hline 07/27/00 & 12:30:55 & 283.4862 & 8.765 & 29.88 & 3.431 \\
\hline 07/27/00 & 12:31:10 & 283.7362 & 8.715 & 29.88 & 3.481 \\
\hline 07/27/00 & $12: 31: 25$ & 283.9862 & 8.711 & 29.88 & 3.485 \\
\hline 07/27/00 & $12: 31: 40$ & 284.2362 & 9.235 & 29.882 & 2.961 \\
\hline 07/27/00 & $12: 31: 55$ & 284.4862 & 9.734 & 29.88 & 2.462 \\
\hline 07/27/00 & 12:32:10 & 284.7362 & 9.896 & 29.88 & 2.3 \\
\hline 07/27/00 & 12:32:25 & 284.9862 & 10.03 & 29.882 & 2.166 \\
\hline 07/27/00 & 12:32:40 & 285.2362 & 10.145 & 29.882 & 2.051 \\
\hline 07/27/00 & 12:32:55 & 285.4862 & 10.249 & 29.88 & 1.947 \\
\hline 07/27/00 & $12: 33: 10$ & 285.7362 & 10.341 & 29.882 & 1.855 \\
\hline 07/27/00 & 12:33:25 & 285.9862 & 10.42 & 29.88 & 1.776 \\
\hline 07/27/00 & 12:33:40 & 286.2362 & 10.493 & 29.882 & 1.703 \\
\hline 07/27/00 & 12:33:55 & 286.4862 & 10.557 & 29.88 & 1.639 \\
\hline 07/27/00 & 12:34:10 & 286.7362 & 10.616 & 29.88 & 1.58 \\
\hline 07/27/00 & 12:34:25 & 286.9862 & 10.669 & 29.878 & 1.527 \\
\hline 07/27/00 & $12: 34: 40$ & 287.2362 & 10.717 & 29.88 & 1.479 \\
\hline 07/27/00 & $12: 34: 55$ & 287.4862 & 10.76 & 29.88 & 1.436 \\
\hline 07/27/00 & 12:35:10 & 287.7362 & 10.799 & 29.88 & 1.397 \\
\hline 07/27/00 & 12:35:25 & 287.9862 & 10.836 & 29.878 & 1.36 \\
\hline 07/27/00 & 12:35:40 & 288.2362 & 10.871 & 29.88 & 1.325 \\
\hline 07/27/00 & 12:35:55 & 288.4862 & 10.901 & 29.878 & 1.295 \\
\hline
\end{tabular}




\section{Appendix B (continued)}

\begin{tabular}{|c|c|c|c|c|c|}
\hline $07 / 27 / 00$ & $12: 36: 10$ & 288.7362 & 10.931 & 29.878 & 1.265 \\
\hline $07 / 27 / 00$ & $12: 36: 25$ & 288.9862 & 10.962 & 29.88 & 1.234 \\
\hline $07 / 27 / 00$ & $12: 36: 40$ & 289.2362 & 10.988 & 29.88 & 1.208 \\
\hline $07 / 27 / 00$ & 12:36:55 & 289.4862 & 11.012 & 29.878 & 1.184 \\
\hline $07 / 27 / 00$ & $12: 37: 10$ & 289.7362 & 11.037 & 29.88 & 1.159 \\
\hline $07 / 27 / 00$ & 12:37:25 & 289.9862 & 11.058 & 29.878 & 1.138 \\
\hline $07 / 27 / 00$ & $12: 37: 40$ & 290.2362 & 11.08 & 29.876 & 1.116 \\
\hline $07 / 27 / 00$ & $12: 37: 55$ & 290.4862 & 11.098 & 29.876 & 1.098 \\
\hline $07 / 27 / 00$ & 12:38:10 & 290.7362 & 11.119 & 29.878 & 1.077 \\
\hline $07 / 27 / 00$ & $12: 38: 25$ & 290.9862 & 11.136 & 29.878 & 1.06 \\
\hline $07 / 27 / 00$ & $12: 38: 40$ & 291.2362 & 11.155 & 29.876 & 1.041 \\
\hline $07 / 27 / 00$ & 12:38:55 & 291.4862 & 11.172 & 29.876 & 1.024 \\
\hline $07 / 27 / 00$ & 12:39:10 & 291.7362 & 11.186 & 29.878 & 1.01 \\
\hline $07 / 27 / 00$ & $12: 39: 25$ & 291.9862 & 11.204 & 29.878 & 0.992 \\
\hline $07 / 27 / 00$ & 12:39:40 & 292.2362 & 11.217 & 29.878 & 0.979 \\
\hline $07 / 27 / 00$ & 12:39:55 & 292.4862 & 11.231 & 29.878 & 0.965 \\
\hline $07 / 27 / 00$ & $12: 40: 10$ & 292.7362 & 11.247 & 29.878 & 0.949 \\
\hline $07 / 27 / 00$ & $12: 40: 25$ & 292.9862 & 11.258 & 29.878 & 0.938 \\
\hline $07 / 27 / 00$ & $12: 40: 40$ & 293.2362 & 11.27 & 29.88 & 0.926 \\
\hline $07 / 27 / 00$ & $12: 40: 55$ & 293.4862 & 11.284 & 29.878 & 0.912 \\
\hline $07 / 27 / 00$ & $12: 41: 10$ & 293.7362 & 11.294 & 29.878 & 0.902 \\
\hline $07 / 27 / 00$ & $12: 41: 25$ & 293.9862 & 11.306 & 29.876 & 0.89 \\
\hline $07 / 27 / 00$ & $12: 41: 40$ & 294.2362 & 11.317 & 29.878 & 0.879 \\
\hline $07 / 27 / 00$ & 12:41:55 & 294.4862 & 11.329 & 29.878 & 0.867 \\
\hline $07 / 27 / 00$ & 12:42:10 & 294.7362 & 11.339 & 29.876 & 0.857 \\
\hline $07 / 27 / 00$ & $12: 42: 25$ & 294.9862 & 11.349 & 29.876 & 0.847 \\
\hline $07 / 27 / 00$ & 12:42:40 & 295.2362 & 11.358 & 29.876 & 0.838 \\
\hline $07 / 27 / 00$ & 12:42:55 & 295.4862 & 11.369 & 29.876 & 0.827 \\
\hline $07 / 27 / 00$ & 12:43:10 & 295.7362 & 11.378 & 29.876 & 0.818 \\
\hline $07 / 27 / 00$ & 12:43:25 & 295.9862 & 11.387 & 29.876 & 0.809 \\
\hline $07 / 27 / 00$ & 12:43:40 & 296.2362 & 11.394 & 29.876 & 0.802 \\
\hline $07 / 27 / 00$ & 12:43:55 & 296.4862 & 11.404 & 29.876 & 0.792 \\
\hline $07 / 27 / 00$ & $12: 44: 10$ & 296.7362 & 11.413 & 29.876 & 0.783 \\
\hline $07 / 27 / 00$ & $12: 44: 25$ & 296.9862 & 11.42 & 29.876 & 0.776 \\
\hline $07 / 27 / 00$ & 12:44:40 & 297.2362 & 11.427 & 29.876 & 0.769 \\
\hline $07 / 27 / 00$ & 12:44:55 & 297.4862 & 11.434 & 29.876 & 0.762 \\
\hline $07 / 27 / 00$ & $12: 45: 10$ & 297.7362 & 11.441 & 29.876 & 0.755 \\
\hline $07 / 27 / 00$ & $12: 45: 25$ & 297.9862 & 11.45 & 29.874 & 0.746 \\
\hline $07 / 27 / 00$ & 12:45:40 & 298.2362 & 11.457 & 29.876 & 0.739 \\
\hline $07 / 27 / 00$ & $12: 45: 55$ & 298.4862 & 11.464 & 29.878 & 0.732 \\
\hline $07 / 27 / 00$ & $12: 46: 10$ & 298.7362 & 11.472 & 29.874 & 0.724 \\
\hline $07 / 27 / 00$ & $12: 46: 25$ & 298.9862 & 11.479 & 29.874 & 0.717 \\
\hline $07 / 27 / 00$ & $12: 46: 40$ & 299.2362 & 11.485 & 29.874 & 0.711 \\
\hline $07 / 27 / 00$ & $12: 46: 55$ & 299.4862 & 11.493 & 29.874 & 0.703 \\
\hline $07 / 27 / 00$ & $12: 47: 10$ & 299.7362 & 11.499 & 29.874 & 0.697 \\
\hline $07 / 27 / 00$ & $12: 47: 25$ & 299.9862 & 11.505 & 29.874 & 0.691 \\
\hline $07 / 27 / 00$ & $12: 47: 40$ & 300.2362 & 11.51 & 29.874 & 0.686 \\
\hline $07 / 27 / 00$ & 12:47:55 & 300.4862 & 11.516 & 29.874 & 0.68 \\
\hline $07 / 27 / 00$ & 12:48:10 & 300.7362 & 11.522 & 29.872 & 0.674 \\
\hline $07 / 27 / 00$ & 12:48:25 & 300.9862 & 11.528 & 29.874 & 0.668 \\
\hline $07 / 27 / 00$ & $12: 48: 40$ & 301.2362 & 11.535 & 29.874 & 0.661 \\
\hline
\end{tabular}




\begin{tabular}{|c|c|c|c|c|c|}
\hline \multicolumn{6}{|c|}{ Appendix B (continued) } \\
\hline $07 / 27 / 00$ & $12: 48: 55$ & 301.4862 & 11.539 & 29.874 & 0.657 \\
\hline $07 / 27 / 00$ & $12: 49: 10$ & 301.7362 & 11.545 & 29.872 & 0.651 \\
\hline $07 / 27 / 00$ & $12: 49: 25$ & 301.9862 & 11.551 & 29.874 & 0.645 \\
\hline $07 / 27 / 00$ & $12: 49: 40$ & 302.2362 & 11.555 & 29.874 & 0.641 \\
\hline 07/27/00 & 12:49:55 & 302.4862 & 11.559 & 29.872 & 0.637 \\
\hline $07 / 27 / 00$ & $12: 50: 10$ & 302.7362 & 11.565 & 29.874 & 0.631 \\
\hline $07 / 27 / 00$ & $12: 50: 25$ & 302.9862 & 11.57 & 29.874 & 0.626 \\
\hline 07/27/00 & $12: 50: 40$ & 303.2362 & 11.575 & 29.874 & 0.621 \\
\hline $07 / 27 / 00$ & $12: 50: 55$ & 303.4862 & 11.58 & 29.872 & 0.616 \\
\hline $07 / 27 / 00$ & $12: 51: 10$ & 303.7362 & 11.584 & 29.872 & 0.612 \\
\hline $07 / 27 / 00$ & $12: 51: 25$ & 303.9862 & 11.585 & 29.886 & 0.611 \\
\hline $07 / 27 / 00$ & $12: 51: 40$ & 304.2362 & 11.59 & 29.886 & 0.606 \\
\hline 07/27/00 & $12: 51: 55$ & 304.4862 & 11.597 & 29.876 & 0.599 \\
\hline 07/27/00 & 12:52:10 & 304.7362 & 11.601 & 29.874 & 0.595 \\
\hline $07 / 27 / 00$ & 12:52:25 & 304.9862 & 11.606 & 29.872 & 0.59 \\
\hline $07 / 27 / 00$ & $12: 52: 40$ & 305.2362 & 11.61 & 29.872 & 0.586 \\
\hline 07/27/00 & 12:52:55 & 305.4862 & 11.613 & 29.872 & 0.583 \\
\hline $07 / 27 / 00$ & $12: 53: 10$ & 305.7362 & 11.619 & 29.872 & 0.577 \\
\hline $07 / 27 / 00$ & 12:53:25 & 305.9862 & 11.624 & 29.872 & 0.572 \\
\hline 07/27/00 & $12: 53: 40$ & 306.2362 & 11.627 & 29.872 & 0.569 \\
\hline $07 / 27 / 00$ & 12:53:55 & 306.4862 & 11.631 & 29.87 & 0.565 \\
\hline $07 / 27 / 00$ & $12: 54: 10$ & 306.7362 & 11.634 & 29.872 & 0.562 \\
\hline $07 / 27 / 00$ & $12: 54: 25$ & 306.9862 & 11.639 & 29.872 & 0.557 \\
\hline $07 / 27 / 00$ & $12: 54: 40$ & 307.2362 & 11.643 & 29.872 & 0.553 \\
\hline $07 / 27 / 00$ & $12: 54: 55$ & 307.4862 & 11.644 & 29.874 & 0.552 \\
\hline 07/27/00 & $12: 55: 10$ & 307.7362 & 11.649 & 29.872 & 0.547 \\
\hline $07 / 27 / 00$ & $12: 55: 25$ & 307.9862 & 11.653 & 29.872 & 0.543 \\
\hline $07 / 27 / 00$ & $12: 55: 40$ & 308.2362 & 11.656 & 29.872 & 0.54 \\
\hline $07 / 27 / 00$ & 12:55:55 & 308.4862 & 11.662 & 29.87 & 0.534 \\
\hline $07 / 27 / 00$ & $12: 56: 10$ & 308.7362 & 11.666 & 29.874 & 0.53 \\
\hline $07 / 27 / 00$ & $12: 56: 25$ & 308.9862 & 11.668 & 29.872 & 0.528 \\
\hline $07 / 27 / 00$ & $12: 56: 40$ & 309.2362 & 11.672 & 29.87 & 0.524 \\
\hline $07 / 27 / 00$ & $12: 56: 55$ & 309.4862 & 11.675 & 29.872 & 0.521 \\
\hline $07 / 27 / 00$ & $12: 57: 10$ & 309.7362 & 11.678 & 29.87 & 0.518 \\
\hline $07 / 27 / 00$ & $12: 57: 25$ & 309.9862 & 11.682 & 29.868 & 0.514 \\
\hline $07 / 27 / 00$ & 12:57:40 & 310.2362 & 11.685 & 29.87 & 0.511 \\
\hline $07 / 27 / 00$ & $12: 57: 55$ & 310.4862 & 11.688 & 29.87 & 0.508 \\
\hline 07/27/00 & $12: 58: 10$ & 310.7362 & 11.691 & 29.87 & 0.505 \\
\hline 07/27/00 & $12: 58: 25$ & 310.9862 & 11.693 & 29.87 & 0.503 \\
\hline $07 / 27 / 00$ & $12: 58: 40$ & 311.2362 & 11.696 & 29.87 & 0.5 \\
\hline $07 / 27 / 00$ & $12: 58: 55$ & 311.4862 & 11.701 & 29.87 & 0.495 \\
\hline $07 / 27 / 00$ & $12: 59: 10$ & 311.7362 & 11.704 & 29.87 & 0.492 \\
\hline
\end{tabular}




\section{Appendix B (continued)}

This page intentionally left blank 


\section{Appendix C - Time-drawdown data for SWP-100D}

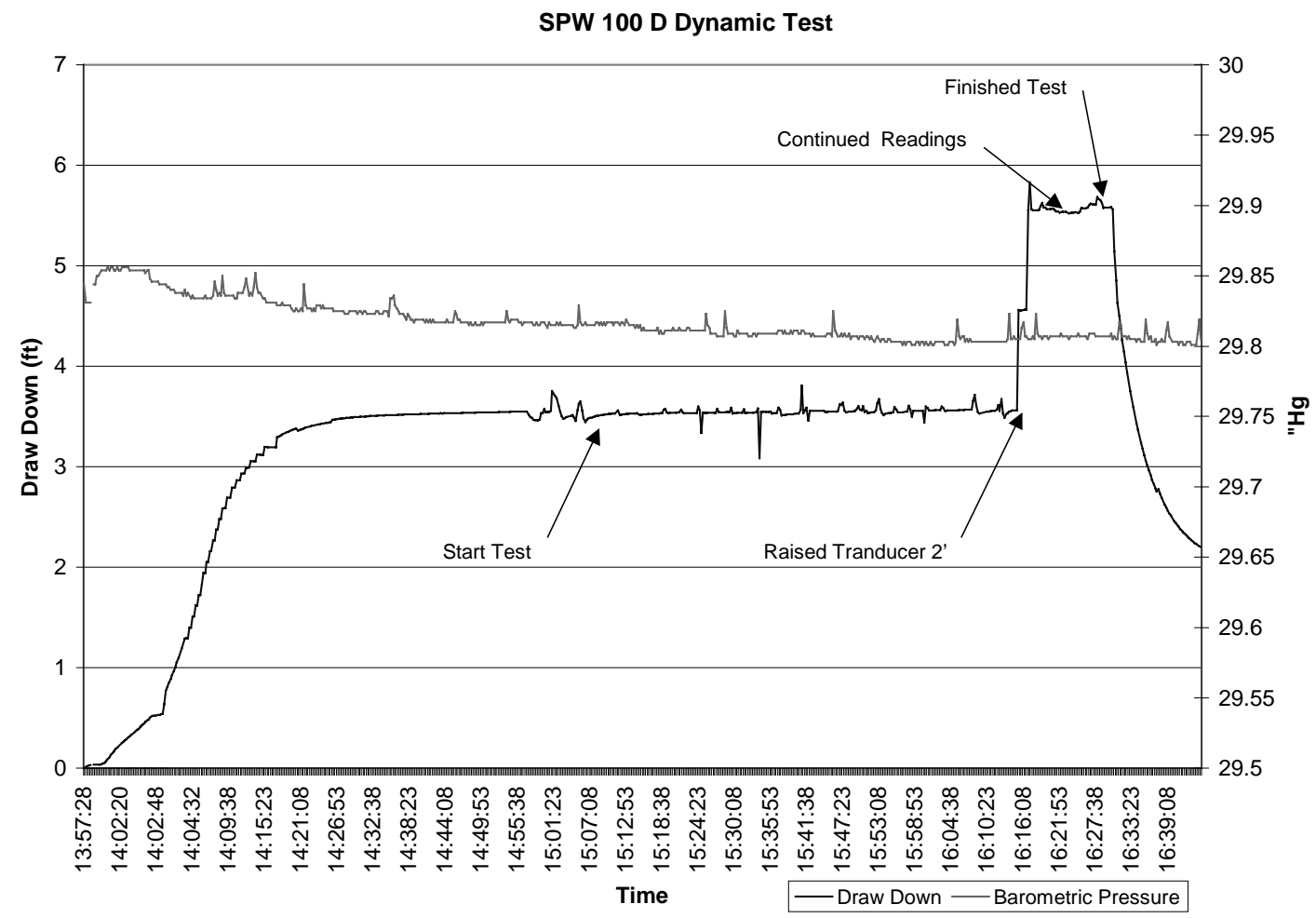

Back Ground

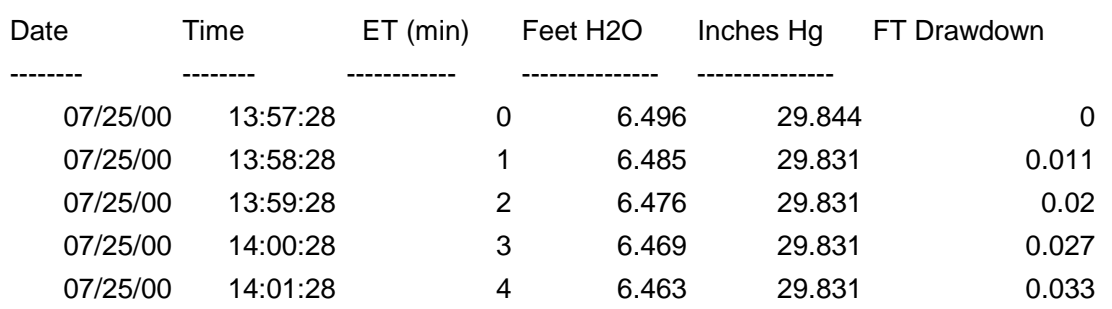

Pumping

$\begin{array}{lrrrrr}07 / 25 / 00 & 14: 02: 09 & 0 & 6.46 & 29.844 & 0.036 \\ 07 / 25 / 00 & 14: 02: 09 & 0.0112 & 6.46 & 29.844 & 0.036 \\ 07 / 25 / 00 & 14: 02: 10 & 0.0223 & 6.46 & 29.85 & 0.036 \\ 07 / 25 / 00 & 14: 02: 11 & 0.0335 & 6.459 & 29.85 & 0.037 \\ 07 / 25 / 00 & 14: 02: 11 & 0.0447 & 6.459 & 29.852 & 0.037 \\ 07 / 25 / 00 & 14: 02: 12 & 0.0558 & 6.456 & 29.854 & 0.04 \\ 07 / 25 / 00 & 14: 02: 13 & 0.067 & 6.449 & 29.854 & 0.047 \\ 07 / 25 / 00 & 14: 02: 13 & 0.0782 & 6.439 & 29.854 & 0.057 \\ 07 / 25 / 00 & 14: 02: 14 & 0.0893 & 6.425 & 29.854 & 0.071 \\ 07 / 25 / 00 & 14: 02: 15 & 0.1005 & 6.406 & 29.856 & 0.09 \\ 07 / 25 / 00 & 14: 02: 15 & 0.1117 & 6.387 & 29.854 & 0.109 \\ 07 / 25 / 00 & 14: 02: 16 & 0.1228 & 6.364 & 29.856 & 0.132 \\ 07 / 25 / 00 & 14: 02: 17 & 0.134 & 6.344 & 29.856 & 0.152 \\ 07 / 25 / 00 & 14: 02: 17 & 0.1452 & 6.324 & 29.854 & 0.172\end{array}$




\section{Appendix C (continued)}

\begin{tabular}{|c|c|c|c|c|c|}
\hline $07 / 25 / 00$ & 14:02:18 & 0.1563 & 6.306 & 29.854 & 0.19 \\
\hline $07 / 25 / 00$ & 14:02:19 & 0.1675 & 6.289 & 29.856 & 0.207 \\
\hline $07 / 25 / 00$ & $14: 02: 19$ & 0.1787 & 6.275 & 29.854 & 0.221 \\
\hline $07 / 25 / 00$ & 14:02:20 & 0.1898 & 6.26 & 29.854 & 0.236 \\
\hline $07 / 25 / 00$ & $14: 02: 21$ & 0.201 & 6.245 & 29.856 & 0.251 \\
\hline $07 / 25 / 00$ & 14:02:21 & 0.2122 & 6.232 & 29.856 & 0.264 \\
\hline $07 / 25 / 00$ & 14:02:22 & 0.2233 & 6.219 & 29.856 & 0.277 \\
\hline $07 / 25 / 00$ & $14: 02: 23$ & 0.235 & 6.204 & 29.856 & 0.292 \\
\hline $07 / 25 / 00$ & $14: 02: 23$ & 0.2475 & 6.191 & 29.856 & 0.305 \\
\hline $07 / 25 / 00$ & $14: 02: 24$ & 0.2607 & 6.18 & 29.854 & 0.316 \\
\hline $07 / 25 / 00$ & $14: 02: 25$ & 0.2747 & 6.164 & 29.854 & 0.332 \\
\hline $07 / 25 / 00$ & $14: 02: 26$ & 0.2895 & 6.152 & 29.854 & 0.344 \\
\hline $07 / 25 / 00$ & 14:02:27 & 0.3052 & 6.14 & 29.854 & 0.356 \\
\hline $07 / 25 / 00$ & 14:02:28 & 0.3218 & 6.125 & 29.854 & 0.371 \\
\hline $07 / 25 / 00$ & 14:02:29 & 0.3395 & 6.111 & 29.854 & 0.385 \\
\hline $07 / 25 / 00$ & 14:02:30 & 0.3582 & 6.096 & 29.854 & 0.4 \\
\hline $07 / 25 / 00$ & 14:02:31 & 0.378 & 6.081 & 29.854 & 0.415 \\
\hline $07 / 25 / 00$ & 14:02:32 & 0.399 & 6.066 & 29.854 & 0.43 \\
\hline $07 / 25 / 00$ & 14:02:34 & 0.4212 & 6.052 & 29.854 & 0.444 \\
\hline $07 / 25 / 00$ & 14:02:35 & 0.4447 & 6.034 & 29.852 & 0.462 \\
\hline $07 / 25 / 00$ & $14: 02: 37$ & 0.4695 & 6.019 & 29.854 & 0.477 \\
\hline $07 / 25 / 00$ & $14: 02: 38$ & 0.4958 & 6.011 & 29.854 & 0.485 \\
\hline $07 / 25 / 00$ & $14: 02: 40$ & 0.5238 & 5.99 & 29.848 & 0.506 \\
\hline $07 / 25 / 00$ & 14:02:42 & 0.5535 & 5.978 & 29.846 & 0.518 \\
\hline $07 / 25 / 00$ & 14:02:44 & 0.5848 & 5.974 & 29.846 & 0.522 \\
\hline $07 / 25 / 00$ & 14:02:46 & 0.618 & 5.971 & 29.846 & 0.525 \\
\hline $07 / 25 / 00$ & 14:02:48 & 0.6532 & 5.97 & 29.846 & 0.526 \\
\hline $07 / 25 / 00$ & $14: 02: 50$ & 0.6905 & 5.964 & 29.846 & 0.532 \\
\hline $07 / 25 / 00$ & 14:02:52 & 0.73 & 5.963 & 29.844 & 0.533 \\
\hline $07 / 25 / 00$ & $14: 02: 55$ & 0.7718 & 5.96 & 29.844 & 0.536 \\
\hline $07 / 25 / 00$ & 14:02:57 & 0.8162 & 5.955 & 29.844 & 0.541 \\
\hline $07 / 25 / 00$ & 14:03:00 & 0.8632 & 5.856 & 29.844 & 0.64 \\
\hline $07 / 25 / 00$ & 14:03:03 & 0.913 & 5.724 & 29.844 & 0.772 \\
\hline $07 / 25 / 00$ & 14:03:06 & 0.9657 & 5.685 & 29.842 & 0.811 \\
\hline $07 / 25 / 00$ & 14:03:10 & 1.0215 & 5.646 & 29.842 & 0.85 \\
\hline $07 / 25 / 00$ & 14:03:13 & 1.0807 & 5.608 & 29.84 & 0.888 \\
\hline $07 / 25 / 00$ & 14:03:17 & 1.1433 & 5.568 & 29.84 & 0.928 \\
\hline $07 / 25 / 00$ & 14:03:21 & 1.2097 & 5.528 & 29.84 & 0.968 \\
\hline $07 / 25 / 00$ & $14: 03: 25$ & 1.28 & 5.486 & 29.838 & 1.01 \\
\hline $07 / 25 / 00$ & 14:03:30 & 1.3545 & 5.443 & 29.838 & 1.053 \\
\hline $07 / 25 / 00$ & 14:03:35 & 1.4335 & 5.398 & 29.838 & 1.098 \\
\hline $07 / 25 / 00$ & 14:03:40 & 1.5172 & 5.352 & 29.838 & 1.144 \\
\hline $07 / 25 / 00$ & 14:03:45 & 1.6057 & 5.306 & 29.838 & 1.19 \\
\hline $07 / 25 / 00$ & 14:03:50 & 1.6995 & 5.256 & 29.836 & 1.24 \\
\hline $07 / 25 / 00$ & 14:03:56 & 1.7988 & 5.206 & 29.84 & 1.29 \\
\hline $07 / 25 / 00$ & $14: 04: 03$ & 1.9042 & 5.201 & 29.836 & 1.295 \\
\hline $07 / 25 / 00$ & $14: 04: 09$ & 2.0157 & 5.2 & 29.838 & 1.296 \\
\hline $07 / 25 / 00$ & $14: 04: 17$ & 2.1338 & 5.095 & 29.836 & 1.401 \\
\hline $07 / 25 / 00$ & $14: 04: 24$ & 2.259 & 5.095 & 29.834 & 1.401 \\
\hline $07 / 25 / 00$ & 14:04:32 & 2.3915 & 4.987 & 29.836 & 1.509 \\
\hline $07 / 25 / 00$ & $14: 04: 40$ & 2.532 & 4.985 & 29.834 & 1.511 \\
\hline
\end{tabular}




\begin{tabular}{|c|c|c|c|c|c|}
\hline \multicolumn{5}{|c|}{ Appendix C (continued) } & \multirow[b]{2}{*}{1.617} \\
\hline 07/25/00 & $14: 04: 49$ & 2.6808 & 4.879 & 29.834 & \\
\hline $07 / 25 / 00$ & $14: 04: 59$ & 2.8383 & 4.877 & 29.834 & 1.619 \\
\hline $07 / 25 / 00$ & 14:05:09 & 3.0052 & 4.771 & 29.834 & 1.725 \\
\hline $07 / 25 / 00$ & $14: 05: 19$ & 3.182 & 4.771 & 29.834 & 1.725 \\
\hline $07 / 25 / 00$ & $14: 05: 31$ & 3.3693 & 4.663 & 29.834 & 1.833 \\
\hline $07 / 25 / 00$ & $14: 05: 43$ & 3.5677 & 4.555 & 29.834 & 1.941 \\
\hline 07/25/00 & $14: 05: 55$ & 3.7778 & 4.554 & 29.836 & 1.942 \\
\hline $07 / 25 / 00$ & 14:06:09 & 4.0005 & 4.446 & 29.834 & 2.05 \\
\hline $07 / 25 / 00$ & $14: 06: 23$ & 4.2363 & 4.444 & 29.834 & 2.052 \\
\hline $07 / 25 / 00$ & 14:06:38 & 4.4862 & 4.336 & 29.834 & 2.16 \\
\hline $07 / 25 / 00$ & $14: 06: 53$ & 4.7362 & 4.335 & 29.834 & 2.161 \\
\hline $07 / 25 / 00$ & 14:07:08 & 4.9862 & 4.229 & 29.836 & 2.267 \\
\hline $07 / 25 / 00$ & $14: 07: 23$ & 5.2362 & 4.227 & 29.846 & 2.269 \\
\hline $07 / 25 / 00$ & $14: 07: 38$ & 5.4862 & 4.119 & 29.84 & 2.377 \\
\hline 07/25/00 & $14: 07: 53$ & 5.7362 & 4.119 & 29.836 & 2.377 \\
\hline $07 / 25 / 00$ & 14:08:08 & 5.9862 & 4.016 & 29.838 & 2.48 \\
\hline 07/25/00 & 14:08:23 & 6.2362 & 4.014 & 29.836 & 2.482 \\
\hline $07 / 25 / 00$ & 14:08:38 & 6.4862 & 3.911 & 29.85 & 2.585 \\
\hline $07 / 25 / 00$ & 14:08:53 & 6.7362 & 3.909 & 29.838 & 2.587 \\
\hline 07/25/00 & 14:09:08 & 6.9862 & 3.908 & 29.836 & 2.588 \\
\hline 07/25/00 & 14:09:23 & 7.2362 & 3.806 & 29.836 & 2.69 \\
\hline $07 / 25 / 00$ & 14:09:38 & 7.4862 & 3.803 & 29.836 & 2.693 \\
\hline 07/25/00 & $14: 09: 53$ & 7.7362 & 3.803 & 29.836 & 2.693 \\
\hline $07 / 25 / 00$ & $14: 10: 08$ & 7.9862 & 3.702 & 29.836 & 2.794 \\
\hline $07 / 25 / 00$ & $14: 10: 23$ & 8.2362 & 3.702 & 29.836 & 2.794 \\
\hline 07/25/00 & $14: 10: 38$ & 8.4862 & 3.702 & 29.834 & 2.794 \\
\hline $07 / 25 / 00$ & $14: 10: 53$ & 8.7362 & 3.629 & 29.834 & 2.867 \\
\hline $07 / 25 / 00$ & 14:11:08 & 8.9862 & 3.629 & 29.838 & 2.867 \\
\hline $07 / 25 / 00$ & $14: 11: 23$ & 9.2362 & 3.629 & 29.838 & 2.867 \\
\hline 07/25/00 & $14: 11: 38$ & 9.4862 & 3.564 & 29.838 & 2.932 \\
\hline 07/25/00 & $14: 11: 53$ & 9.7362 & 3.564 & 29.838 & 2.932 \\
\hline $07 / 25 / 00$ & $14: 12: 08$ & 9.9862 & 3.562 & 29.842 & 2.934 \\
\hline $07 / 25 / 00$ & $14: 12: 23$ & 10.2362 & 3.505 & 29.848 & 2.991 \\
\hline 07/25/00 & $14: 12: 38$ & 10.4862 & 3.508 & 29.842 & 2.988 \\
\hline $07 / 25 / 00$ & $14: 12: 53$ & 10.7362 & 3.505 & 29.836 & 2.991 \\
\hline $07 / 25 / 00$ & $14: 13: 08$ & 10.9862 & 3.442 & 29.838 & 3.054 \\
\hline $07 / 25 / 00$ & $14: 13: 23$ & 11.2362 & 3.442 & 29.836 & 3.054 \\
\hline 07/25/00 & $14: 13: 38$ & 11.4862 & 3.442 & 29.842 & 3.054 \\
\hline $07 / 25 / 00$ & $14: 13: 53$ & 11.7362 & 3.446 & 29.852 & 3.05 \\
\hline 07/25/00 & $14: 14: 08$ & 11.9862 & 3.375 & 29.842 & 3.121 \\
\hline 07/25/00 & $14: 14: 23$ & 12.2362 & 3.375 & 29.838 & 3.121 \\
\hline $07 / 25 / 00$ & $14: 14: 38$ & 12.4862 & 3.375 & 29.838 & 3.121 \\
\hline $07 / 25 / 00$ & $14: 14: 53$ & 12.7362 & 3.377 & 29.836 & 3.119 \\
\hline 07/25/00 & $14: 15: 08$ & 12.9862 & 3.377 & 29.834 & 3.119 \\
\hline 07/25/00 & $14: 15: 23$ & 13.2362 & 3.299 & 29.834 & 3.197 \\
\hline $07 / 25 / 00$ & $14: 15: 38$ & 13.4862 & 3.299 & 29.831 & 3.197 \\
\hline $07 / 25 / 00$ & $14: 15: 53$ & 13.7362 & 3.301 & 29.831 & 3.195 \\
\hline $07 / 25 / 00$ & $14: 16: 08$ & 13.9862 & 3.301 & 29.831 & 3.195 \\
\hline $07 / 25 / 00$ & $14: 16: 23$ & 14.2362 & 3.301 & 29.831 & 3.195 \\
\hline $07 / 25 / 00$ & $14: 16: 38$ & 14.4862 & 3.301 & 29.831 & 3.195 \\
\hline $07 / 25 / 00$ & $14: 16: 53$ & 14.7362 & 3.302 & 29.831 & 3.194 \\
\hline
\end{tabular}




\section{Appendix C (continued)}

\begin{tabular}{|c|c|c|c|c|c|}
\hline $07 / 25 / 00$ & 14:17:08 & 14.9862 & 3.302 & 29.831 & 3.194 \\
\hline $07 / 25 / 00$ & $14: 17: 23$ & 15.2362 & 3.201 & 29.829 & 3.295 \\
\hline $07 / 25 / 00$ & $14: 17: 38$ & 15.4862 & 3.193 & 29.829 & 3.303 \\
\hline $07 / 25 / 00$ & $14: 17: 53$ & 15.7362 & 3.184 & 29.829 & 3.312 \\
\hline $07 / 25 / 00$ & $14: 18: 08$ & 15.9862 & 3.174 & 29.831 & 3.322 \\
\hline $07 / 25 / 00$ & $14: 18: 23$ & 16.2362 & 3.167 & 29.829 & 3.329 \\
\hline $07 / 25 / 00$ & $14: 18: 38$ & 16.4862 & 3.158 & 29.829 & 3.338 \\
\hline $07 / 25 / 00$ & $14: 18: 53$ & 16.7362 & 3.151 & 29.829 & 3.345 \\
\hline $07 / 25 / 00$ & $14: 19: 08$ & 16.9862 & 3.145 & 29.829 & 3.351 \\
\hline $07 / 25 / 00$ & $14: 19: 23$ & 17.2362 & 3.14 & 29.829 & 3.356 \\
\hline $07 / 25 / 00$ & $14: 19: 38$ & 17.4862 & 3.131 & 29.827 & 3.365 \\
\hline $07 / 25 / 00$ & $14: 19: 53$ & 17.7362 & 3.125 & 29.825 & 3.371 \\
\hline $07 / 25 / 00$ & $14: 20: 08$ & 17.9862 & 3.119 & 29.825 & 3.377 \\
\hline $07 / 25 / 00$ & $14: 20: 23$ & 18.2362 & 3.117 & 29.827 & 3.379 \\
\hline $07 / 25 / 00$ & $14: 20: 38$ & 18.4862 & 3.137 & 29.825 & 3.359 \\
\hline $07 / 25 / 00$ & $14: 20: 53$ & 18.7362 & 3.132 & 29.825 & 3.364 \\
\hline $07 / 25 / 00$ & $14: 21: 08$ & 18.9862 & 3.125 & 29.827 & 3.371 \\
\hline $07 / 25 / 00$ & $14: 21: 23$ & 19.2362 & 3.118 & 29.825 & 3.378 \\
\hline $07 / 25 / 00$ & $14: 21: 38$ & 19.4862 & 3.115 & 29.844 & 3.381 \\
\hline $07 / 25 / 00$ & $14: 21: 53$ & 19.7362 & 3.106 & 29.829 & 3.39 \\
\hline $07 / 25 / 00$ & $14: 22: 08$ & 19.9862 & 3.102 & 29.827 & 3.394 \\
\hline $07 / 25 / 00$ & $14: 22: 23$ & 20.2362 & 3.098 & 29.827 & 3.398 \\
\hline $07 / 25 / 00$ & $14: 22: 38$ & 20.4862 & 3.092 & 29.827 & 3.404 \\
\hline $07 / 25 / 00$ & $14: 22: 53$ & 20.7362 & 3.088 & 29.825 & 3.408 \\
\hline $07 / 25 / 00$ & $14: 23: 08$ & 20.9862 & 3.086 & 29.827 & 3.41 \\
\hline $07 / 25 / 00$ & $14: 23: 23$ & 21.2362 & 3.082 & 29.825 & 3.414 \\
\hline $07 / 25 / 00$ & $14: 23: 38$ & 21.4862 & 3.078 & 29.829 & 3.418 \\
\hline $07 / 25 / 00$ & $14: 23: 53$ & 21.7362 & 3.075 & 29.829 & 3.421 \\
\hline $07 / 25 / 00$ & $14: 24: 08$ & 21.9862 & 3.072 & 29.829 & 3.424 \\
\hline 07/25/00 & $14: 24: 23$ & 22.2362 & 3.069 & 29.827 & 3.427 \\
\hline $07 / 25 / 00$ & $14: 24: 38$ & 22.4862 & 3.066 & 29.829 & 3.43 \\
\hline $07 / 25 / 00$ & $14: 24: 53$ & 22.7362 & 3.063 & 29.827 & 3.433 \\
\hline $07 / 25 / 00$ & $14: 25: 08$ & 22.9862 & 3.06 & 29.827 & 3.436 \\
\hline $07 / 25 / 00$ & $14: 25: 23$ & 23.2362 & 3.058 & 29.827 & 3.438 \\
\hline $07 / 25 / 00$ & $14: 25: 38$ & 23.4862 & 3.053 & 29.827 & 3.443 \\
\hline $07 / 25 / 00$ & $14: 25: 53$ & 23.7362 & 3.05 & 29.827 & 3.446 \\
\hline $07 / 25 / 00$ & $14: 26: 08$ & 23.9862 & 3.027 & 29.827 & 3.469 \\
\hline $07 / 25 / 00$ & $14: 26: 23$ & 24.2362 & 3.026 & 29.825 & 3.47 \\
\hline $07 / 25 / 00$ & $14: 26: 38$ & 24.4862 & 3.023 & 29.825 & 3.473 \\
\hline $07 / 25 / 00$ & $14: 26: 53$ & 24.7362 & 3.02 & 29.825 & 3.476 \\
\hline $07 / 25 / 00$ & $14: 27: 08$ & 24.9862 & 3.019 & 29.825 & 3.477 \\
\hline 07/25/00 & $14: 27: 23$ & 25.2362 & 3.017 & 29.825 & 3.479 \\
\hline $07 / 25 / 00$ & $14: 27: 38$ & 25.4862 & 3.016 & 29.825 & 3.48 \\
\hline $07 / 25 / 00$ & $14: 27: 53$ & 25.7362 & 3.014 & 29.825 & 3.482 \\
\hline $07 / 25 / 00$ & $14: 28: 08$ & 25.9862 & 3.012 & 29.823 & 3.484 \\
\hline $07 / 25 / 00$ & $14: 28: 23$ & 26.2362 & 3.01 & 29.823 & 3.486 \\
\hline $07 / 25 / 00$ & $14: 28: 38$ & 26.4862 & 3.007 & 29.823 & 3.489 \\
\hline $07 / 25 / 00$ & $14: 28: 53$ & 26.7362 & 3.006 & 29.825 & 3.49 \\
\hline $07 / 25 / 00$ & $14: 29: 08$ & 26.9862 & 3.004 & 29.825 & 3.492 \\
\hline $07 / 25 / 00$ & $14: 29: 23$ & 27.2362 & 3.004 & 29.825 & 3.492 \\
\hline $07 / 25 / 00$ & $14: 29: 38$ & 27.4862 & 3.001 & 29.825 & 3.495 \\
\hline
\end{tabular}


Appendix C (continued)

\begin{tabular}{|c|c|c|c|c|c|}
\hline $07 / 25 / 00$ & $14: 29: 53$ & 27.7362 & 3.001 & 29.825 & 3.495 \\
\hline 07/25/00 & 14:30:08 & 27.9862 & 3 & 29.823 & 3.496 \\
\hline 07/25/00 & $14: 30: 23$ & 28.2362 & 2.999 & 29.825 & 3.497 \\
\hline $07 / 25 / 00$ & 14:30:38 & 28.4862 & 2.997 & 29.825 & 3.499 \\
\hline 07/25/00 & $14: 30: 53$ & 28.7362 & 2.996 & 29.823 & 3.5 \\
\hline 07/25/00 & 14:31:08 & 28.9862 & 2.996 & 29.825 & 3.5 \\
\hline 07/25/00 & $14: 31: 23$ & 29.2362 & 2.996 & 29.825 & 3.5 \\
\hline 07/25/00 & 14:31:38 & 29.4862 & 2.994 & 29.823 & 3.502 \\
\hline 07/25/00 & $14: 31: 53$ & 29.7362 & 2.993 & 29.823 & 3.503 \\
\hline $07 / 25 / 00$ & 14:32:08 & 29.9862 & 2.991 & 29.823 & 3.505 \\
\hline 07/25/00 & 14:32:23 & 30.2362 & 2.991 & 29.823 & 3.505 \\
\hline 07/25/00 & 14:32:38 & 30.4862 & 2.99 & 29.823 & 3.506 \\
\hline 07/25/00 & 14:32:53 & 30.7362 & 2.99 & 29.823 & 3.506 \\
\hline 07/25/00 & 14:33:08 & 30.9862 & 2.989 & 29.825 & 3.507 \\
\hline 07/25/00 & $14: 33: 23$ & 31.2362 & 2.987 & 29.825 & 3.509 \\
\hline $07 / 25 / 00$ & 14:33:38 & 31.4862 & 2.987 & 29.823 & 3.509 \\
\hline 07/25/00 & 14:33:53 & 31.7362 & 2.986 & 29.823 & 3.51 \\
\hline 07/25/00 & 14:34:08 & 31.9862 & 2.984 & 29.823 & 3.512 \\
\hline 07/25/00 & $14: 34: 23$ & 32.2362 & 2.984 & 29.825 & 3.512 \\
\hline 07/25/00 & 14:34:38 & 32.4862 & 2.983 & 29.825 & 3.513 \\
\hline 07/25/00 & $14: 34: 53$ & 32.7362 & 2.981 & 29.825 & 3.515 \\
\hline $07 / 25 / 00$ & $14: 35: 08$ & 32.9862 & 2.983 & 29.821 & 3.513 \\
\hline 07/25/00 & $14: 35: 23$ & 33.2362 & 2.981 & 29.834 & 3.515 \\
\hline 07/25/00 & 14:35:38 & 33.4862 & 2.98 & 29.834 & 3.516 \\
\hline 07/25/00 & $14: 35: 53$ & 33.7362 & 2.98 & 29.836 & 3.516 \\
\hline 07/25/00 & $14: 36: 08$ & 33.9862 & 2.98 & 29.829 & 3.516 \\
\hline 07/25/00 & $14: 36: 23$ & 34.2362 & 2.98 & 29.827 & 3.516 \\
\hline $07 / 25 / 00$ & 14:36:38 & 34.4862 & 2.978 & 29.825 & 3.518 \\
\hline 07/25/00 & 14:36:53 & 34.7362 & 2.978 & 29.823 & 3.518 \\
\hline 07/25/00 & 14:37:08 & 34.9862 & 2.978 & 29.823 & 3.518 \\
\hline 07/25/00 & 14:37:23 & 35.2362 & 2.977 & 29.823 & 3.519 \\
\hline 07/25/00 & 14:37:38 & 35.4862 & 2.976 & 29.821 & 3.52 \\
\hline 07/25/00 & $14: 37: 53$ & 35.7362 & 2.976 & 29.819 & 3.52 \\
\hline $07 / 25 / 00$ & 14:38:08 & 35.9862 & 2.976 & 29.823 & 3.52 \\
\hline 07/25/00 & $14: 38: 23$ & 36.2362 & 2.976 & 29.821 & 3.52 \\
\hline 07/25/00 & 14:38:38 & 36.4862 & 2.976 & 29.819 & 3.52 \\
\hline 07/25/00 & $14: 38: 53$ & 36.7362 & 2.974 & 29.819 & 3.522 \\
\hline 07/25/00 & 14:39:08 & 36.9862 & 2.976 & 29.817 & 3.52 \\
\hline 07/25/00 & 14:39:23 & 37.2362 & 2.974 & 29.819 & 3.522 \\
\hline $07 / 25 / 00$ & 14:39:38 & 37.4862 & 2.973 & 29.819 & 3.523 \\
\hline 07/25/00 & 14:39:53 & 37.7362 & 2.973 & 29.819 & 3.523 \\
\hline 07/25/00 & 14:40:08 & 37.9862 & 2.971 & 29.819 & 3.525 \\
\hline 07/25/00 & $14: 40: 23$ & 38.2362 & 2.971 & 29.819 & 3.525 \\
\hline 07/25/00 & 14:40:38 & 38.4862 & 2.971 & 29.819 & 3.525 \\
\hline 07/25/00 & $14: 40: 53$ & 38.7362 & 2.971 & 29.817 & 3.525 \\
\hline $07 / 25 / 00$ & $14: 41: 08$ & 38.9862 & 2.97 & 29.819 & 3.526 \\
\hline 07/25/00 & $14: 41: 23$ & 39.2362 & 2.97 & 29.817 & 3.526 \\
\hline 07/25/00 & 14:41:38 & 39.4862 & 2.968 & 29.819 & 3.528 \\
\hline 07/25/00 & $14: 41: 53$ & 39.7362 & 2.968 & 29.817 & 3.528 \\
\hline 07/25/00 & 14:42:08 & 39.9862 & 2.968 & 29.819 & 3.528 \\
\hline 07/25/00 & 14:42:23 & 40.2362 & 2.968 & 29.817 & 3.528 \\
\hline
\end{tabular}




\section{Appendix C (continued)}

\begin{tabular}{|c|c|c|c|c|c|}
\hline $07 / 25 / 00$ & 14:42:38 & 40.4862 & 2.965 & 29.817 & 3.531 \\
\hline $07 / 25 / 00$ & $14: 42: 53$ & 40.7362 & 2.965 & 29.817 & 3.531 \\
\hline $07 / 25 / 00$ & 14:43:08 & 40.9862 & 2.965 & 29.817 & 3.531 \\
\hline $07 / 25 / 00$ & 14:43:23 & 41.2362 & 2.964 & 29.817 & 3.532 \\
\hline $07 / 25 / 00$ & 14:43:38 & 41.4862 & 2.965 & 29.817 & 3.531 \\
\hline $07 / 25 / 00$ & $14: 43: 53$ & 41.7362 & 2.964 & 29.817 & 3.532 \\
\hline $07 / 25 / 00$ & $14: 44: 08$ & 41.9862 & 2.964 & 29.817 & 3.532 \\
\hline $07 / 25 / 00$ & $14: 44: 23$ & 42.2362 & 2.964 & 29.819 & 3.532 \\
\hline $07 / 25 / 00$ & $14: 44: 38$ & 42.4862 & 2.964 & 29.817 & 3.532 \\
\hline $07 / 25 / 00$ & $14: 44: 53$ & 42.7362 & 2.963 & 29.817 & 3.533 \\
\hline 07/25/00 & 14:45:08 & 42.9862 & 2.963 & 29.817 & 3.533 \\
\hline $07 / 25 / 00$ & $14: 45: 23$ & 43.2362 & 2.963 & 29.817 & 3.533 \\
\hline $07 / 25 / 00$ & $14: 45: 38$ & 43.4862 & 2.961 & 29.825 & 3.535 \\
\hline $07 / 25 / 00$ & $14: 45: 53$ & 43.7362 & 2.961 & 29.823 & 3.535 \\
\hline $07 / 25 / 00$ & $14: 46: 08$ & 43.9862 & 2.961 & 29.819 & 3.535 \\
\hline $07 / 25 / 00$ & $14: 46: 23$ & 44.2362 & 2.96 & 29.819 & 3.536 \\
\hline $07 / 25 / 00$ & $14: 46: 38$ & 44.4862 & 2.96 & 29.817 & 3.536 \\
\hline $07 / 25 / 00$ & $14: 46: 53$ & 44.7362 & 2.958 & 29.817 & 3.538 \\
\hline $07 / 25 / 00$ & $14: 47: 08$ & 44.9862 & 2.958 & 29.817 & 3.538 \\
\hline $07 / 25 / 00$ & $14: 47: 23$ & 45.2362 & 2.958 & 29.817 & 3.538 \\
\hline $07 / 25 / 00$ & $14: 47: 38$ & 45.4862 & 2.958 & 29.817 & 3.538 \\
\hline $07 / 25 / 00$ & $14: 47: 53$ & 45.7362 & 2.958 & 29.815 & 3.538 \\
\hline $07 / 25 / 00$ & 14:48:08 & 45.9862 & 2.957 & 29.817 & 3.539 \\
\hline $07 / 25 / 00$ & $14: 48: 23$ & 46.2362 & 2.957 & 29.815 & 3.539 \\
\hline $07 / 25 / 00$ & $14: 48: 38$ & 46.4862 & 2.957 & 29.815 & 3.539 \\
\hline $07 / 25 / 00$ & $14: 48: 53$ & 46.7362 & 2.957 & 29.817 & 3.539 \\
\hline $07 / 25 / 00$ & $14: 49: 08$ & 46.9862 & 2.955 & 29.815 & 3.541 \\
\hline $07 / 25 / 00$ & $14: 49: 23$ & 47.2362 & 2.955 & 29.815 & 3.541 \\
\hline $07 / 25 / 00$ & $14: 49: 38$ & 47.4862 & 2.955 & 29.815 & 3.541 \\
\hline $07 / 25 / 00$ & $14: 49: 53$ & 47.7362 & 2.955 & 29.817 & 3.541 \\
\hline $07 / 25 / 00$ & $14: 50: 08$ & 47.9862 & 2.954 & 29.815 & 3.542 \\
\hline $07 / 25 / 00$ & $14: 50: 23$ & 48.2362 & 2.955 & 29.817 & 3.541 \\
\hline $07 / 25 / 00$ & $14: 50: 38$ & 48.4862 & 2.954 & 29.817 & 3.542 \\
\hline $07 / 25 / 00$ & $14: 50: 53$ & 48.7362 & 2.954 & 29.817 & 3.542 \\
\hline $07 / 25 / 00$ & 14:51:08 & 48.9862 & 2.954 & 29.817 & 3.542 \\
\hline $07 / 25 / 00$ & $14: 51: 23$ & 49.2362 & 2.953 & 29.817 & 3.543 \\
\hline $07 / 25 / 00$ & $14: 51: 38$ & 49.4862 & 2.953 & 29.817 & 3.543 \\
\hline $07 / 25 / 00$ & $14: 51: 53$ & 49.7362 & 2.953 & 29.817 & 3.543 \\
\hline $07 / 25 / 00$ & 14:52:08 & 49.9862 & 2.953 & 29.817 & 3.543 \\
\hline $07 / 25 / 00$ & $14: 52: 23$ & 50.2362 & 2.953 & 29.817 & 3.543 \\
\hline $07 / 25 / 00$ & 14:52:38 & 50.4862 & 2.951 & 29.817 & 3.545 \\
\hline $07 / 25 / 00$ & 14:52:53 & 50.7362 & 2.951 & 29.817 & 3.545 \\
\hline $07 / 25 / 00$ & 14:53:08 & 50.9862 & 2.951 & 29.817 & 3.545 \\
\hline $07 / 25 / 00$ & $14: 53: 23$ & 51.2362 & 2.95 & 29.817 & 3.546 \\
\hline $07 / 25 / 00$ & 14:53:38 & 51.4862 & 2.95 & 29.817 & 3.546 \\
\hline $07 / 25 / 00$ & $14: 53: 53$ & 51.7362 & 2.95 & 29.825 & 3.546 \\
\hline $07 / 25 / 00$ & 14:54:08 & 51.9862 & 2.95 & 29.819 & 3.546 \\
\hline 07/25/00 & $14: 54: 23$ & 52.2362 & 2.95 & 29.817 & 3.546 \\
\hline $07 / 25 / 00$ & $14: 54: 38$ & 52.4862 & 2.948 & 29.819 & 3.548 \\
\hline $07 / 25 / 00$ & $14: 54: 53$ & 52.7362 & 2.948 & 29.819 & 3.548 \\
\hline $07 / 25 / 00$ & $14: 55: 08$ & 52.9862 & 2.948 & 29.819 & 3.548 \\
\hline
\end{tabular}




\section{Appendix C (continued)}

\begin{tabular}{|c|c|c|c|c|c|}
\hline $07 / 25 / 00$ & $14: 55: 23$ & 53.2362 & 2.948 & 29.819 & 3.548 \\
\hline $07 / 25 / 00$ & $14: 55: 38$ & 53.4862 & 2.948 & 29.819 & 3.548 \\
\hline $07 / 25 / 00$ & $14: 55: 53$ & 53.7362 & 2.947 & 29.817 & 3.549 \\
\hline 07/25/00 & 14:56:08 & 53.9862 & 2.947 & 29.817 & 3.549 \\
\hline $07 / 25 / 00$ & $14: 56: 23$ & 54.2362 & 2.945 & 29.815 & 3.551 \\
\hline $07 / 25 / 00$ & $14: 56: 38$ & 54.4862 & 2.945 & 29.817 & 3.551 \\
\hline $07 / 25 / 00$ & $14: 56: 53$ & 54.7362 & 2.945 & 29.817 & 3.551 \\
\hline $07 / 25 / 00$ & 14:57:08 & 54.9862 & 2.945 & 29.817 & 3.551 \\
\hline $07 / 25 / 00$ & $14: 57: 23$ & 55.2362 & 2.977 & 29.817 & 3.519 \\
\hline $07 / 25 / 00$ & 14:57:38 & 55.4862 & 3.003 & 29.817 & 3.493 \\
\hline $07 / 25 / 00$ & $14: 57: 53$ & 55.7362 & 3.009 & 29.817 & 3.487 \\
\hline $07 / 25 / 00$ & 14:58:08 & 55.9862 & 3.026 & 29.815 & 3.47 \\
\hline $07 / 25 / 00$ & 14:58:23 & 56.2362 & 3.032 & 29.815 & 3.464 \\
\hline $07 / 25 / 00$ & 14:58:38 & 56.4862 & 3.032 & 29.817 & 3.464 \\
\hline $07 / 25 / 00$ & $14: 58: 53$ & 56.7362 & 3.036 & 29.815 & 3.46 \\
\hline $07 / 25 / 00$ & 14:59:08 & 56.9862 & 3.029 & 29.815 & 3.467 \\
\hline $07 / 25 / 00$ & $14: 59: 23$ & 57.2362 & 2.961 & 29.817 & 3.535 \\
\hline $07 / 25 / 00$ & 14:59:38 & 57.4862 & 2.96 & 29.817 & 3.536 \\
\hline $07 / 25 / 00$ & $14: 59: 53$ & 57.7362 & 2.921 & 29.817 & 3.575 \\
\hline $07 / 25 / 00$ & 15:00:08 & 57.9862 & 2.953 & 29.815 & 3.543 \\
\hline $07 / 25 / 00$ & $15: 00: 23$ & 58.2362 & 2.951 & 29.813 & 3.545 \\
\hline $07 / 25 / 00$ & 15:00:38 & 58.4862 & 2.951 & 29.817 & 3.545 \\
\hline $07 / 25 / 00$ & 15:00:53 & 58.7362 & 2.938 & 29.815 & 3.558 \\
\hline $07 / 25 / 00$ & 15:01:08 & 58.9862 & 2.74 & 29.815 & 3.756 \\
\hline $07 / 25 / 00$ & 15:01:23 & 59.2362 & 2.77 & 29.815 & 3.726 \\
\hline $07 / 25 / 00$ & 15:01:38 & 59.4862 & 2.796 & 29.815 & 3.7 \\
\hline $07 / 25 / 00$ & 15:01:53 & 59.7362 & 2.814 & 29.817 & 3.682 \\
\hline $07 / 25 / 00$ & 15:02:08 & 59.9862 & 2.888 & 29.815 & 3.608 \\
\hline $07 / 25 / 00$ & $15: 02: 23$ & 60.2362 & 2.945 & 29.817 & 3.551 \\
\hline $07 / 25 / 00$ & 15:02:38 & 60.4862 & 2.994 & 29.815 & 3.502 \\
\hline $07 / 25 / 00$ & 15:02:53 & 60.7362 & 3.016 & 29.815 & 3.48 \\
\hline $07 / 25 / 00$ & 15:03:08 & 60.9862 & 3.012 & 29.815 & 3.484 \\
\hline $07 / 25 / 00$ & $15: 03: 23$ & 61.2362 & 3.003 & 29.815 & 3.493 \\
\hline $07 / 25 / 00$ & 15:03:38 & 61.4862 & 2.997 & 29.815 & 3.499 \\
\hline $07 / 25 / 00$ & 15:03:53 & 61.7362 & 2.993 & 29.815 & 3.503 \\
\hline $07 / 25 / 00$ & 15:04:08 & 61.9862 & 2.989 & 29.815 & 3.507 \\
\hline $07 / 25 / 00$ & $15: 04: 23$ & 62.2362 & 2.981 & 29.815 & 3.515 \\
\hline $07 / 25 / 00$ & 15:04:38 & 62.4862 & 3.007 & 29.813 & 3.489 \\
\hline $07 / 25 / 00$ & $15: 04: 53$ & 62.7362 & 3.04 & 29.813 & 3.456 \\
\hline $07 / 25 / 00$ & 15:05:08 & 62.9862 & 2.932 & 29.815 & 3.564 \\
\hline $07 / 25 / 00$ & $15: 05: 23$ & 63.2362 & 2.868 & 29.829 & 3.628 \\
\hline $07 / 25 / 00$ & 15:05:38 & 63.4862 & 2.845 & 29.817 & 3.651 \\
\hline $07 / 25 / 00$ & 15:05:53 & 63.7362 & 2.942 & 29.815 & 3.554 \\
\hline $07 / 25 / 00$ & 15:06:08 & 63.9862 & 3.012 & 29.815 & 3.484 \\
\hline $07 / 25 / 00$ & $15: 06: 23$ & 64.2362 & 3.05 & 29.817 & 3.446 \\
\hline $07 / 25 / 00$ & $15: 06: 38$ & 64.4862 & 3.026 & 29.815 & 3.47 \\
\hline $07 / 25 / 00$ & $15: 06: 53$ & 64.7362 & 3.017 & 29.815 & 3.479 \\
\hline $07 / 25 / 00$ & 15:07:08 & 64.9862 & 3.01 & 29.815 & 3.486 \\
\hline $07 / 25 / 00$ & $15: 07: 23$ & 65.2362 & 3.006 & 29.815 & 3.49 \\
\hline $07 / 25 / 00$ & 15:07:38 & 65.4862 & 3 & 29.815 & 3.496 \\
\hline $07 / 25 / 00$ & 15:07:53 & 65.7362 & 2.996 & 29.815 & 3.5 \\
\hline
\end{tabular}




\begin{tabular}{|c|c|c|c|c|c|}
\hline \multicolumn{5}{|c|}{ Appendix C (continued) } & \multirow[b]{2}{*}{3.505} \\
\hline 07/25/00 & 15:08:08 & 65.9862 & 2.991 & 29.817 & \\
\hline 07/25/00 & $15: 08: 23$ & 66.2362 & 2.989 & 29.817 & 3.507 \\
\hline $07 / 25 / 00$ & 15:08:38 & 66.4862 & 2.986 & 29.815 & 3.51 \\
\hline 07/25/00 & $15: 08: 53$ & 66.7362 & 2.983 & 29.817 & 3.513 \\
\hline 07/25/00 & 15:09:08 & 66.9862 & 2.98 & 29.815 & 3.516 \\
\hline 07/25/00 & $15: 09: 23$ & 67.2362 & 2.977 & 29.817 & 3.519 \\
\hline $07 / 25 / 00$ & $15: 09: 38$ & 67.4862 & 2.976 & 29.815 & 3.52 \\
\hline 07/25/00 & $15: 09: 53$ & 67.7362 & 2.973 & 29.817 & 3.523 \\
\hline 07/25/00 & $15: 10: 08$ & 67.9862 & 2.971 & 29.817 & 3.525 \\
\hline $07 / 25 / 00$ & $15: 10: 23$ & 68.2362 & 2.97 & 29.817 & 3.526 \\
\hline 07/25/00 & $15: 10: 38$ & 68.4862 & 2.967 & 29.817 & 3.529 \\
\hline 07/25/00 & $15: 10: 53$ & 68.7362 & 2.965 & 29.815 & 3.531 \\
\hline $07 / 25 / 00$ & $15: 11: 08$ & 68.9862 & 2.965 & 29.817 & 3.531 \\
\hline 07/25/00 & $15: 11: 23$ & 69.2362 & 2.948 & 29.817 & 3.548 \\
\hline $07 / 25 / 00$ & 15:11:38 & 69.4862 & 2.937 & 29.817 & 3.559 \\
\hline 07/25/00 & $15: 11: 53$ & 69.7362 & 2.98 & 29.815 & 3.516 \\
\hline 07/25/00 & 15:12:08 & 69.9862 & 2.977 & 29.817 & 3.519 \\
\hline 07/25/00 & $15: 12: 23$ & 70.2362 & 2.976 & 29.817 & 3.52 \\
\hline 07/25/00 & $15: 12: 38$ & 70.4862 & 2.973 & 29.815 & 3.523 \\
\hline 07/25/00 & $15: 12: 53$ & 70.7362 & 2.971 & 29.819 & 3.525 \\
\hline 07/25/00 & $15: 13: 08$ & 70.9862 & 2.97 & 29.817 & 3.526 \\
\hline 07/25/00 & $15: 13: 23$ & 71.2362 & 2.968 & 29.815 & 3.528 \\
\hline 07/25/00 & 15:13:38 & 71.4862 & 2.968 & 29.815 & 3.528 \\
\hline 07/25/00 & $15: 13: 53$ & 71.7362 & 2.967 & 29.815 & 3.529 \\
\hline $07 / 25 / 00$ & $15: 14: 08$ & 71.9862 & 2.965 & 29.815 & 3.531 \\
\hline 07/25/00 & $15: 14: 23$ & 72.2362 & 2.965 & 29.813 & 3.531 \\
\hline 07/25/00 & 15:14:38 & 72.4862 & 2.964 & 29.815 & 3.532 \\
\hline 07/25/00 & $15: 14: 53$ & 72.7362 & 2.976 & 29.813 & 3.52 \\
\hline 07/25/00 & $15: 15: 08$ & 72.9862 & 2.978 & 29.815 & 3.518 \\
\hline 07/25/00 & $15: 15: 23$ & 73.2362 & 2.977 & 29.811 & 3.519 \\
\hline 07/25/00 & 15:15:38 & 73.4862 & 2.976 & 29.813 & 3.52 \\
\hline 07/25/00 & $15: 15: 53$ & 73.7362 & 2.974 & 29.811 & 3.522 \\
\hline 07/25/00 & $15: 16: 08$ & 73.9862 & 2.973 & 29.811 & 3.523 \\
\hline 07/25/00 & $15: 16: 23$ & 74.2362 & 2.971 & 29.811 & 3.525 \\
\hline 07/25/00 & $15: 16: 38$ & 74.4862 & 2.971 & 29.811 & 3.525 \\
\hline 07/25/00 & $15: 16: 53$ & 74.7362 & 2.971 & 29.811 & 3.525 \\
\hline $07 / 25 / 00$ & $15: 17: 08$ & 74.9862 & 2.97 & 29.811 & 3.526 \\
\hline 07/25/00 & $15: 17: 23$ & 75.2362 & 2.968 & 29.811 & 3.528 \\
\hline 07/25/00 & $15: 17: 38$ & 75.4862 & 2.967 & 29.811 & 3.529 \\
\hline 07/25/00 & 15:17:53 & 75.7362 & 2.967 & 29.813 & 3.529 \\
\hline 07/25/00 & 15:18:08 & 75.9862 & 2.965 & 29.809 & 3.531 \\
\hline 07/25/00 & $15: 18: 23$ & 76.2362 & 2.964 & 29.811 & 3.532 \\
\hline 07/25/00 & 15:18:38 & 76.4862 & 2.964 & 29.809 & 3.532 \\
\hline 07/25/00 & 15:18:53 & 76.7362 & 2.927 & 29.809 & 3.569 \\
\hline 07/25/00 & 15:19:08 & 76.9862 & 2.918 & 29.811 & 3.578 \\
\hline 07/25/00 & $15: 19: 23$ & 77.2362 & 2.955 & 29.811 & 3.541 \\
\hline 07/25/00 & $15: 19: 38$ & 77.4862 & 2.96 & 29.811 & 3.536 \\
\hline 07/25/00 & $15: 19: 53$ & 77.7362 & 2.958 & 29.811 & 3.538 \\
\hline $07 / 25 / 00$ & $15: 20: 08$ & 77.9862 & 2.958 & 29.811 & 3.538 \\
\hline 07/25/00 & 15:20:23 & 78.2362 & 2.958 & 29.813 & 3.538 \\
\hline 07/25/00 & $15: 20: 38$ & 78.4862 & 2.957 & 29.811 & 3.539 \\
\hline
\end{tabular}


Appendix C (continued)

\begin{tabular}{|c|c|c|c|c|c|}
\hline $07 / 25 / 00$ & $15: 20: 53$ & 78.7362 & 2.957 & 29.811 & 3.539 \\
\hline $07 / 25 / 00$ & $15: 21: 08$ & 78.9862 & 2.955 & 29.811 & 3.541 \\
\hline $07 / 25 / 00$ & $15: 21: 23$ & 79.2362 & 2.955 & 29.813 & 3.541 \\
\hline $07 / 25 / 00$ & $15: 21: 38$ & 79.4862 & 2.93 & 29.811 & 3.566 \\
\hline $07 / 25 / 00$ & $15: 21: 53$ & 79.7362 & 2.955 & 29.811 & 3.541 \\
\hline $07 / 25 / 00$ & $15: 22: 08$ & 79.9862 & 2.964 & 29.811 & 3.532 \\
\hline $07 / 25 / 00$ & $15: 22: 23$ & 80.2362 & 2.964 & 29.811 & 3.532 \\
\hline $07 / 25 / 00$ & $15: 22: 38$ & 80.4862 & 2.963 & 29.813 & 3.533 \\
\hline $07 / 25 / 00$ & $15: 22: 53$ & 80.7362 & 2.963 & 29.813 & 3.533 \\
\hline $07 / 25 / 00$ & $15: 23: 08$ & 80.9862 & 2.963 & 29.811 & 3.533 \\
\hline $07 / 25 / 00$ & $15: 23: 23$ & 81.2362 & 2.963 & 29.811 & 3.533 \\
\hline $07 / 25 / 00$ & $15: 23: 38$ & 81.4862 & 2.963 & 29.811 & 3.533 \\
\hline $07 / 25 / 00$ & $15: 23: 53$ & 81.7362 & 2.963 & 29.811 & 3.533 \\
\hline $07 / 25 / 00$ & $15: 24: 08$ & 81.9862 & 2.963 & 29.811 & 3.533 \\
\hline $07 / 25 / 00$ & $15: 24: 23$ & 82.2362 & 2.896 & 29.811 & 3.6 \\
\hline $07 / 25 / 00$ & $15: 24: 38$ & 82.4862 & 2.937 & 29.811 & 3.559 \\
\hline $07 / 25 / 00$ & $15: 24: 53$ & 82.7362 & 3.158 & 29.811 & 3.338 \\
\hline $07 / 25 / 00$ & $15: 25: 08$ & 82.9862 & 2.958 & 29.811 & 3.538 \\
\hline $07 / 25 / 00$ & $15: 25: 23$ & 83.2362 & 2.958 & 29.811 & 3.538 \\
\hline $07 / 25 / 00$ & $15: 25: 38$ & 83.4862 & 2.963 & 29.823 & 3.533 \\
\hline $07 / 25 / 00$ & $15: 25: 53$ & 83.7362 & 2.958 & 29.815 & 3.538 \\
\hline $07 / 25 / 00$ & $15: 26: 08$ & 83.9862 & 2.958 & 29.813 & 3.538 \\
\hline $07 / 25 / 00$ & $15: 26: 23$ & 84.2362 & 2.958 & 29.809 & 3.538 \\
\hline $07 / 25 / 00$ & $15: 26: 38$ & 84.4862 & 2.957 & 29.809 & 3.539 \\
\hline $07 / 25 / 00$ & $15: 26: 53$ & 84.7362 & 2.958 & 29.809 & 3.538 \\
\hline $07 / 25 / 00$ & $15: 27: 08$ & 84.9862 & 2.963 & 29.809 & 3.533 \\
\hline $07 / 25 / 00$ & $15: 27: 23$ & 85.2362 & 2.919 & 29.807 & 3.577 \\
\hline $07 / 25 / 00$ & $15: 27: 38$ & 85.4862 & 2.951 & 29.807 & 3.545 \\
\hline $07 / 25 / 00$ & $15: 27: 53$ & 85.7362 & 2.953 & 29.807 & 3.543 \\
\hline $07 / 25 / 00$ & $15: 28: 08$ & 85.9862 & 2.953 & 29.807 & 3.543 \\
\hline $07 / 25 / 00$ & $15: 28: 23$ & 86.2362 & 2.953 & 29.807 & 3.543 \\
\hline $07 / 25 / 00$ & $15: 28: 38$ & 86.4862 & 2.955 & 29.825 & 3.541 \\
\hline $07 / 25 / 00$ & $15: 28: 53$ & 86.7362 & 2.951 & 29.813 & 3.545 \\
\hline $07 / 25 / 00$ & $15: 29: 08$ & 86.9862 & 2.951 & 29.809 & 3.545 \\
\hline $07 / 25 / 00$ & $15: 29: 23$ & 87.2362 & 2.954 & 29.809 & 3.542 \\
\hline $07 / 25 / 00$ & $15: 29: 38$ & 87.4862 & 2.909 & 29.809 & 3.587 \\
\hline $07 / 25 / 00$ & $15: 29: 53$ & 87.7362 & 2.965 & 29.809 & 3.531 \\
\hline $07 / 25 / 00$ & $15: 30: 08$ & 87.9862 & 2.963 & 29.807 & 3.533 \\
\hline $07 / 25 / 00$ & $15: 30: 23$ & 88.2362 & 2.963 & 29.807 & 3.533 \\
\hline $07 / 25 / 00$ & $15: 30: 38$ & 88.4862 & 2.958 & 29.811 & 3.538 \\
\hline $07 / 25 / 00$ & $15: 30: 53$ & 88.7362 & 2.957 & 29.809 & 3.539 \\
\hline $07 / 25 / 00$ & 15:31:08 & 88.9862 & 2.958 & 29.809 & 3.538 \\
\hline $07 / 25 / 00$ & $15: 31: 23$ & 89.2362 & 2.958 & 29.809 & 3.538 \\
\hline $07 / 25 / 00$ & $15: 31: 38$ & 89.4862 & 2.924 & 29.809 & 3.572 \\
\hline $07 / 25 / 00$ & $15: 31: 53$ & 89.7362 & 2.961 & 29.809 & 3.535 \\
\hline $07 / 25 / 00$ & $15: 32: 08$ & 89.9862 & 2.958 & 29.809 & 3.538 \\
\hline $07 / 25 / 00$ & $15: 32: 23$ & 90.2362 & 2.958 & 29.807 & 3.538 \\
\hline $07 / 25 / 00$ & $15: 32: 38$ & 90.4862 & 2.957 & 29.807 & 3.539 \\
\hline $07 / 25 / 00$ & 15:32:53 & 90.7362 & 2.957 & 29.807 & 3.539 \\
\hline $07 / 25 / 00$ & $15: 33: 08$ & 90.9862 & 2.957 & 29.809 & 3.539 \\
\hline $07 / 25 / 00$ & $15: 33: 23$ & 91.2362 & 2.955 & 29.809 & 3.541 \\
\hline
\end{tabular}




\begin{tabular}{|c|c|c|c|c|c|}
\hline \multicolumn{5}{|c|}{ Appendix $\mathbf{C}$ (continued) } & \multirow[b]{2}{*}{3.545} \\
\hline 07/25/00 & 15:33:38 & 91.4862 & 2.951 & 29.807 & \\
\hline $07 / 25 / 00$ & $15: 33: 53$ & 91.7362 & 2.915 & 29.809 & 3.581 \\
\hline 07/25/00 & $15: 34: 08$ & 91.9862 & 3.41 & 29.809 & 3.086 \\
\hline 07/25/00 & $15: 34: 23$ & 92.2362 & 2.951 & 29.809 & 3.545 \\
\hline $07 / 25 / 00$ & $15: 34: 38$ & 92.4862 & 2.95 & 29.809 & 3.546 \\
\hline 07/25/00 & $15: 34: 53$ & 92.7362 & 2.948 & 29.809 & 3.548 \\
\hline $07 / 25 / 00$ & $15: 35: 08$ & 92.9862 & 2.95 & 29.809 & 3.546 \\
\hline 07/25/00 & $15: 35: 23$ & 93.2362 & 2.951 & 29.809 & 3.545 \\
\hline 07/25/00 & $15: 35: 38$ & 93.4862 & 2.95 & 29.809 & 3.546 \\
\hline $07 / 25 / 00$ & $15: 35: 53$ & 93.7362 & 2.948 & 29.809 & 3.548 \\
\hline 07/25/00 & $15: 36: 08$ & 93.9862 & 2.965 & 29.809 & 3.531 \\
\hline $07 / 25 / 00$ & $15: 36: 23$ & 94.2362 & 2.963 & 29.809 & 3.533 \\
\hline $07 / 25 / 00$ & $15: 36: 38$ & 94.4862 & 2.963 & 29.809 & 3.533 \\
\hline 07/25/00 & $15: 36: 53$ & 94.7362 & 2.963 & 29.809 & 3.533 \\
\hline $07 / 25 / 00$ & 15:37:08 & 94.9862 & 2.908 & 29.811 & 3.588 \\
\hline $07 / 25 / 00$ & $15: 37: 23$ & 95.2362 & 2.927 & 29.811 & 3.569 \\
\hline $07 / 25 / 00$ & $15: 37: 38$ & 95.4862 & 2.987 & 29.811 & 3.509 \\
\hline $07 / 25 / 00$ & $15: 37: 53$ & 95.7362 & 2.981 & 29.809 & 3.515 \\
\hline $07 / 25 / 00$ & 15:38:08 & 95.9862 & 2.98 & 29.811 & 3.516 \\
\hline $07 / 25 / 00$ & $15: 38: 23$ & 96.2362 & 2.977 & 29.809 & 3.519 \\
\hline $07 / 25 / 00$ & $15: 38: 38$ & 96.4862 & 2.976 & 29.809 & 3.52 \\
\hline $07 / 25 / 00$ & $15: 38: 53$ & 96.7362 & 2.974 & 29.809 & 3.522 \\
\hline $07 / 25 / 00$ & $15: 39: 08$ & 96.9862 & 2.973 & 29.811 & 3.523 \\
\hline $07 / 25 / 00$ & $15: 39: 23$ & 97.2362 & 2.973 & 29.809 & 3.523 \\
\hline $07 / 25 / 00$ & 15:39:38 & 97.4862 & 2.971 & 29.811 & 3.525 \\
\hline $07 / 25 / 00$ & $15: 39: 53$ & 97.7362 & 2.968 & 29.811 & 3.528 \\
\hline $07 / 25 / 00$ & $15: 40: 08$ & 97.9862 & 2.967 & 29.811 & 3.529 \\
\hline 07/25/00 & $15: 40: 23$ & 98.2362 & 2.967 & 29.809 & 3.529 \\
\hline $07 / 25 / 00$ & $15: 40: 38$ & 98.4862 & 2.931 & 29.809 & 3.565 \\
\hline $07 / 25 / 00$ & $15: 40: 53$ & 98.7362 & 2.691 & 29.811 & 3.805 \\
\hline 07/25/00 & $15: 41: 08$ & 98.9862 & 2.963 & 29.811 & 3.533 \\
\hline $07 / 25 / 00$ & $15: 41: 23$ & 99.2362 & 2.932 & 29.809 & 3.564 \\
\hline $07 / 25 / 00$ & $15: 41: 38$ & 99.4862 & 2.909 & 29.809 & 3.587 \\
\hline $07 / 25 / 00$ & $15: 41: 53$ & 99.7362 & 3.037 & 29.809 & 3.459 \\
\hline $07 / 25 / 00$ & $15: 42: 08$ & 99.9862 & 2.942 & 29.809 & 3.554 \\
\hline $07 / 25 / 00$ & $15: 42: 23$ & 100.2362 & 2.941 & 29.807 & 3.555 \\
\hline $07 / 25 / 00$ & $15: 42: 38$ & 100.4862 & 2.941 & 29.809 & 3.555 \\
\hline $07 / 25 / 00$ & $15: 42: 53$ & 100.7362 & 2.941 & 29.807 & 3.555 \\
\hline 07/25/00 & $15: 43: 08$ & 100.9862 & 2.941 & 29.807 & 3.555 \\
\hline $07 / 25 / 00$ & $15: 43: 23$ & 101.2362 & 2.94 & 29.807 & 3.556 \\
\hline $07 / 25 / 00$ & $15: 43: 38$ & 101.4862 & 2.94 & 29.807 & 3.556 \\
\hline $07 / 25 / 00$ & $15: 43: 53$ & 101.7362 & 2.94 & 29.807 & 3.556 \\
\hline $07 / 25 / 00$ & $15: 44: 08$ & 101.9862 & 2.94 & 29.807 & 3.556 \\
\hline $07 / 25 / 00$ & $15: 44: 23$ & 102.2362 & 2.94 & 29.807 & 3.556 \\
\hline $07 / 25 / 00$ & $15: 44: 38$ & 102.4862 & 2.95 & 29.807 & 3.546 \\
\hline $07 / 25 / 00$ & $15: 44: 53$ & 102.7362 & 2.95 & 29.809 & 3.546 \\
\hline $07 / 25 / 00$ & $15: 45: 08$ & 102.9862 & 2.948 & 29.809 & 3.548 \\
\hline $07 / 25 / 00$ & $15: 45: 23$ & 103.2362 & 2.947 & 29.809 & 3.549 \\
\hline $07 / 25 / 00$ & $15: 45: 38$ & 103.4862 & 2.947 & 29.807 & 3.549 \\
\hline $07 / 25 / 00$ & $15: 45: 53$ & 103.7362 & 2.948 & 29.825 & 3.548 \\
\hline $07 / 25 / 00$ & 15:46:08 & 103.9862 & 2.945 & 29.811 & 3.551 \\
\hline
\end{tabular}


Appendix C (continued)

\begin{tabular}{|c|c|c|c|c|c|}
\hline $07 / 25 / 00$ & $15: 46: 23$ & 104.2362 & 2.945 & 29.809 & 3.551 \\
\hline $07 / 25 / 00$ & $15: 46: 38$ & 104.4862 & 2.944 & 29.807 & 3.552 \\
\hline $07 / 25 / 00$ & $15: 46: 53$ & 104.7362 & 2.876 & 29.807 & 3.62 \\
\hline $07 / 25 / 00$ & $15: 47: 08$ & 104.9862 & 2.881 & 29.809 & 3.615 \\
\hline $07 / 25 / 00$ & $15: 47: 23$ & 105.2362 & 2.859 & 29.807 & 3.637 \\
\hline $07 / 25 / 00$ & $15: 47: 38$ & 105.4862 & 2.937 & 29.809 & 3.559 \\
\hline $07 / 25 / 00$ & $15: 47: 53$ & 105.7362 & 2.953 & 29.807 & 3.543 \\
\hline $07 / 25 / 00$ & $15: 48: 08$ & 105.9862 & 2.95 & 29.807 & 3.546 \\
\hline $07 / 25 / 00$ & $15: 48: 23$ & 106.2362 & 2.948 & 29.809 & 3.548 \\
\hline $07 / 25 / 00$ & $15: 48: 38$ & 106.4862 & 2.947 & 29.805 & 3.549 \\
\hline $07 / 25 / 00$ & $15: 48: 53$ & 106.7362 & 2.945 & 29.807 & 3.551 \\
\hline $07 / 25 / 00$ & $15: 49: 08$ & 106.9862 & 2.944 & 29.807 & 3.552 \\
\hline $07 / 25 / 00$ & $15: 49: 23$ & 107.2362 & 2.942 & 29.807 & 3.554 \\
\hline $07 / 25 / 00$ & $15: 49: 38$ & 107.4862 & 2.921 & 29.807 & 3.575 \\
\hline $07 / 25 / 00$ & $15: 49: 53$ & 107.7362 & 2.894 & 29.805 & 3.602 \\
\hline $07 / 25 / 00$ & $15: 50: 08$ & 107.9862 & 2.93 & 29.807 & 3.566 \\
\hline $07 / 25 / 00$ & $15: 50: 23$ & 108.2362 & 2.935 & 29.807 & 3.561 \\
\hline $07 / 25 / 00$ & $15: 50: 38$ & 108.4862 & 2.895 & 29.807 & 3.601 \\
\hline $07 / 25 / 00$ & $15: 50: 53$ & 108.7362 & 2.95 & 29.805 & 3.546 \\
\hline $07 / 25 / 00$ & $15: 51: 08$ & 108.9862 & 2.942 & 29.807 & 3.554 \\
\hline $07 / 25 / 00$ & $15: 51: 23$ & 109.2362 & 2.961 & 29.807 & 3.535 \\
\hline $07 / 25 / 00$ & $15: 51: 38$ & 109.4862 & 2.958 & 29.805 & 3.538 \\
\hline $07 / 25 / 00$ & $15: 51: 53$ & 109.7362 & 2.955 & 29.807 & 3.541 \\
\hline $07 / 25 / 00$ & 15:52:08 & 109.9862 & 2.953 & 29.805 & 3.543 \\
\hline $07 / 25 / 00$ & $15: 52: 23$ & 110.2362 & 2.951 & 29.803 & 3.545 \\
\hline $07 / 25 / 00$ & $15: 52: 38$ & 110.4862 & 2.937 & 29.805 & 3.559 \\
\hline $07 / 25 / 00$ & $15: 52: 53$ & 110.7362 & 2.86 & 29.807 & 3.636 \\
\hline $07 / 25 / 00$ & $15: 53: 08$ & 110.9862 & 2.822 & 29.805 & 3.674 \\
\hline $07 / 25 / 00$ & $15: 53: 23$ & 111.2362 & 2.918 & 29.803 & 3.578 \\
\hline $07 / 25 / 00$ & $15: 53: 38$ & 111.4862 & 2.964 & 29.805 & 3.532 \\
\hline $07 / 25 / 00$ & 15:53:53 & 111.7362 & 2.98 & 29.805 & 3.516 \\
\hline $07 / 25 / 00$ & $15: 54: 08$ & 111.9862 & 2.971 & 29.803 & 3.525 \\
\hline $07 / 25 / 00$ & $15: 54: 23$ & 112.2362 & 2.968 & 29.803 & 3.528 \\
\hline $07 / 25 / 00$ & $15: 54: 38$ & 112.4862 & 2.964 & 29.805 & 3.532 \\
\hline $07 / 25 / 00$ & $15: 54: 53$ & 112.7362 & 2.961 & 29.805 & 3.535 \\
\hline $07 / 25 / 00$ & 15:55:08 & 112.9862 & 2.958 & 29.803 & 3.538 \\
\hline $07 / 25 / 00$ & $15: 55: 23$ & 113.2362 & 2.955 & 29.803 & 3.541 \\
\hline $07 / 25 / 00$ & $15: 55: 38$ & 113.4862 & 2.902 & 29.803 & 3.594 \\
\hline $07 / 25 / 00$ & $15: 55: 53$ & 113.7362 & 2.927 & 29.803 & 3.569 \\
\hline $07 / 25 / 00$ & $15: 56: 08$ & 113.9862 & 2.954 & 29.803 & 3.542 \\
\hline $07 / 25 / 00$ & $15: 56: 23$ & 114.2362 & 2.955 & 29.803 & 3.541 \\
\hline $07 / 25 / 00$ & 15:56:38 & 114.4862 & 2.954 & 29.803 & 3.542 \\
\hline $07 / 25 / 00$ & $15: 56: 53$ & 114.7362 & 2.953 & 29.801 & 3.543 \\
\hline $07 / 25 / 00$ & 15:57:08 & 114.9862 & 2.95 & 29.803 & 3.546 \\
\hline $07 / 25 / 00$ & $15: 57: 23$ & 115.2362 & 2.948 & 29.801 & 3.548 \\
\hline $07 / 25 / 00$ & $15: 57: 38$ & 115.4862 & 2.948 & 29.803 & 3.548 \\
\hline $07 / 25 / 00$ & $15: 57: 53$ & 115.7362 & 2.888 & 29.803 & 3.608 \\
\hline $07 / 25 / 00$ & 15:58:08 & 115.9862 & 2.928 & 29.803 & 3.568 \\
\hline $07 / 25 / 00$ & $15: 58: 23$ & 116.2362 & 2.996 & 29.801 & 3.5 \\
\hline $07 / 25 / 00$ & $15: 58: 38$ & 116.4862 & 2.942 & 29.801 & 3.554 \\
\hline $07 / 25 / 00$ & $15: 58: 53$ & 116.7362 & 2.942 & 29.801 & 3.554 \\
\hline
\end{tabular}




\begin{tabular}{|c|c|c|c|c|c|}
\hline \multicolumn{5}{|c|}{ Appendix C (continued) } & \multirow[b]{2}{*}{3.555} \\
\hline $07 / 25 / 00$ & 15:59:08 & 116.9862 & 2.941 & 29.803 & \\
\hline $07 / 25 / 00$ & $15: 59: 23$ & 117.2362 & 2.941 & 29.803 & 3.555 \\
\hline 07/25/00 & $15: 59: 38$ & 117.4862 & 2.94 & 29.801 & 3.556 \\
\hline $07 / 25 / 00$ & $15: 59: 53$ & 117.7362 & 2.94 & 29.803 & 3.556 \\
\hline 07/25/00 & 16:00:08 & 117.9862 & 2.94 & 29.803 & 3.556 \\
\hline 07/25/00 & $16: 00: 23$ & 118.2362 & 3.055 & 29.801 & 3.441 \\
\hline $07 / 25 / 00$ & $16: 00: 38$ & 118.4862 & 2.896 & 29.803 & 3.6 \\
\hline 07/25/00 & $16: 00: 53$ & 118.7362 & 2.912 & 29.801 & 3.584 \\
\hline 07/25/00 & 16:01:08 & 118.9862 & 2.935 & 29.801 & 3.561 \\
\hline 07/25/00 & $16: 01: 23$ & 119.2362 & 2.935 & 29.803 & 3.561 \\
\hline 07/25/00 & $16: 01: 38$ & 119.4862 & 2.934 & 29.801 & 3.562 \\
\hline 07/25/00 & $16: 01: 53$ & 119.7362 & 2.934 & 29.801 & 3.562 \\
\hline $07 / 25 / 00$ & $16: 02: 08$ & 119.9862 & 2.932 & 29.803 & 3.564 \\
\hline 07/25/00 & $16: 02: 23$ & 120.2362 & 2.932 & 29.803 & 3.564 \\
\hline 07/25/00 & $16: 02: 38$ & 120.4862 & 2.931 & 29.803 & 3.565 \\
\hline $07 / 25 / 00$ & $16: 02: 53$ & 120.7362 & 2.931 & 29.803 & 3.565 \\
\hline 07/25/00 & 16:03:08 & 120.9862 & 2.896 & 29.803 & 3.6 \\
\hline $07 / 25 / 00$ & $16: 03: 23$ & 121.2362 & 2.912 & 29.803 & 3.584 \\
\hline $07 / 25 / 00$ & $16: 03: 38$ & 121.4862 & 2.94 & 29.801 & 3.556 \\
\hline 07/25/00 & $16: 03: 53$ & 121.7362 & 2.938 & 29.801 & 3.558 \\
\hline $07 / 25 / 00$ & $16: 04: 08$ & 121.9862 & 2.937 & 29.801 & 3.559 \\
\hline 07/25/00 & $16: 04: 23$ & 122.2362 & 2.937 & 29.803 & 3.559 \\
\hline 07/25/00 & $16: 04: 38$ & 122.4862 & 2.935 & 29.803 & 3.561 \\
\hline $07 / 25 / 00$ & $16: 04: 53$ & 122.7362 & 2.935 & 29.803 & 3.561 \\
\hline 07/25/00 & $16: 05: 08$ & 122.9862 & 2.935 & 29.801 & 3.561 \\
\hline $07 / 25 / 00$ & $16: 05: 23$ & 123.2362 & 2.934 & 29.803 & 3.562 \\
\hline $07 / 25 / 00$ & $16: 05: 38$ & 123.4862 & 2.937 & 29.819 & 3.559 \\
\hline 07/25/00 & $16: 05: 53$ & 123.7362 & 2.932 & 29.807 & 3.564 \\
\hline 07/25/00 & $16: 06: 08$ & 123.9862 & 2.931 & 29.805 & 3.565 \\
\hline $07 / 25 / 00$ & $16: 06: 23$ & 124.2362 & 2.931 & 29.807 & 3.565 \\
\hline 07/25/00 & $16: 06: 38$ & 124.4862 & 2.931 & 29.807 & 3.565 \\
\hline 07/25/00 & $16: 06: 53$ & 124.7362 & 2.93 & 29.803 & 3.566 \\
\hline 07/25/00 & $16: 07: 08$ & 124.9862 & 2.928 & 29.805 & 3.568 \\
\hline 07/25/00 & $16: 07: 23$ & 125.2362 & 2.927 & 29.803 & 3.569 \\
\hline $07 / 25 / 00$ & $16: 07: 38$ & 125.4862 & 2.927 & 29.805 & 3.569 \\
\hline $07 / 25 / 00$ & $16: 07: 53$ & 125.7362 & 2.928 & 29.803 & 3.568 \\
\hline $07 / 25 / 00$ & 16:08:08 & 125.9862 & 2.839 & 29.803 & 3.657 \\
\hline $07 / 25 / 00$ & $16: 08: 23$ & 126.2362 & 2.783 & 29.803 & 3.713 \\
\hline $07 / 25 / 00$ & $16: 08: 38$ & 126.4862 & 2.886 & 29.803 & 3.61 \\
\hline $07 / 25 / 00$ & $16: 08: 53$ & 126.7362 & 2.951 & 29.803 & 3.545 \\
\hline 07/25/00 & 16:09:08 & 126.9862 & 2.967 & 29.803 & 3.529 \\
\hline 07/25/00 & 16:09:23 & 127.2362 & 2.961 & 29.803 & 3.535 \\
\hline 07/25/00 & $16: 09: 38$ & 127.4862 & 2.957 & 29.803 & 3.539 \\
\hline 07/25/00 & $16: 09: 53$ & 127.7362 & 2.953 & 29.803 & 3.543 \\
\hline 07/25/00 & $16: 10: 08$ & 127.9862 & 2.95 & 29.803 & 3.546 \\
\hline $07 / 25 / 00$ & $16: 10: 23$ & 128.2362 & 2.948 & 29.803 & 3.548 \\
\hline 07/25/00 & $16: 10: 38$ & 128.4862 & 2.947 & 29.803 & 3.549 \\
\hline 07/25/00 & $16: 10: 53$ & 128.7362 & 2.944 & 29.803 & 3.552 \\
\hline 07/25/00 & $16: 11: 08$ & 128.9862 & 2.942 & 29.803 & 3.554 \\
\hline $07 / 25 / 00$ & $16: 11: 23$ & 129.2362 & 2.94 & 29.803 & 3.556 \\
\hline 07/25/00 & $16: 11: 38$ & 129.4862 & 2.938 & 29.803 & 3.558 \\
\hline
\end{tabular}




\section{Appendix C (continued)}

\begin{tabular}{|c|c|c|c|c|c|}
\hline $07 / 25 / 00$ & $16: 11: 53$ & 129.7362 & 2.93 & 29.803 & 3.566 \\
\hline $07 / 25 / 00$ & 16:12:08 & 129.9862 & 2.879 & 29.803 & 3.617 \\
\hline $07 / 25 / 00$ & $16: 12: 23$ & 130.2362 & 2.938 & 29.803 & 3.558 \\
\hline 07/25/00 & $16: 12: 38$ & 130.4862 & 2.823 & 29.803 & 3.673 \\
\hline $07 / 25 / 00$ & $16: 12: 53$ & 130.7362 & 2.965 & 29.803 & 3.531 \\
\hline $07 / 25 / 00$ & $16: 13: 08$ & 130.9862 & 3.01 & 29.803 & 3.486 \\
\hline $07 / 25 / 00$ & $16: 13: 23$ & 131.2362 & 2.976 & 29.803 & 3.52 \\
\hline $07 / 25 / 00$ & $16: 13: 38$ & 131.4862 & 2.963 & 29.805 & 3.533 \\
\hline $07 / 25 / 00$ & $16: 13: 53$ & 131.7362 & 2.948 & 29.823 & 3.548 \\
\hline $07 / 25 / 00$ & $16: 14: 08$ & 131.9862 & 2.944 & 29.805 & 3.552 \\
\hline $07 / 25 / 00$ & $16: 14: 23$ & 132.2362 & 2.937 & 29.805 & 3.559 \\
\hline $07 / 25 / 00$ & $16: 14: 38$ & 132.4862 & 2.937 & 29.807 & 3.559 \\
\hline $07 / 25 / 00$ & $16: 14: 53$ & 132.7362 & 2.934 & 29.805 & 3.562 \\
\hline $07 / 25 / 00$ & $16: 15: 08$ & 132.9862 & 2.934 & 29.805 & 3.562 \\
\hline $07 / 25 / 00$ & $16: 15: 23$ & 133.2362 & 1.936 & 29.805 & 4.56 \\
\hline $07 / 25 / 00$ & $16: 15: 38$ & 133.4862 & 1.939 & 29.805 & 4.557 \\
\hline $07 / 25 / 00$ & $16: 15: 53$ & 133.7362 & 1.939 & 29.813 & 4.557 \\
\hline $07 / 25 / 00$ & $16: 16: 08$ & 133.9862 & 1.936 & 29.817 & 4.56 \\
\hline $07 / 25 / 00$ & $16: 16: 23$ & 134.2362 & 1.934 & 29.807 & 4.562 \\
\hline $07 / 25 / 00$ & $16: 16: 38$ & 134.4862 & 1.934 & 29.807 & 4.562 \\
\hline $07 / 25 / 00$ & $16: 16: 53$ & 134.7362 & 0.943 & 29.805 & 5.553 \\
\hline $07 / 25 / 00$ & $16: 17: 08$ & 134.9862 & 0.666 & 29.805 & 5.83 \\
\hline $07 / 25 / 00$ & $16: 17: 23$ & 135.2362 & 0.933 & 29.805 & 5.563 \\
\hline $07 / 25 / 00$ & $16: 17: 38$ & 135.4862 & 0.942 & 29.807 & 5.554 \\
\hline $07 / 25 / 00$ & $16: 17: 53$ & 135.7362 & 0.943 & 29.805 & 5.553 \\
\hline $07 / 25 / 00$ & $16: 18: 08$ & 135.9862 & 0.945 & 29.823 & 5.551 \\
\hline $07 / 25 / 00$ & $16: 18: 23$ & 136.2362 & 0.942 & 29.809 & 5.554 \\
\hline $07 / 25 / 00$ & $16: 18: 38$ & 136.4862 & 0.941 & 29.807 & 5.555 \\
\hline $07 / 25 / 00$ & $16: 18: 53$ & 136.7362 & 0.902 & 29.807 & 5.594 \\
\hline $07 / 25 / 00$ & $16: 19: 08$ & 136.9862 & 0.869 & 29.805 & 5.627 \\
\hline $07 / 25 / 00$ & $16: 19: 23$ & 137.2362 & 0.915 & 29.807 & 5.581 \\
\hline $07 / 25 / 00$ & $16: 19: 38$ & 137.4862 & 0.915 & 29.807 & 5.581 \\
\hline $07 / 25 / 00$ & $16: 19: 53$ & 137.7362 & 0.931 & 29.807 & 5.565 \\
\hline $07 / 25 / 00$ & $16: 20: 08$ & 137.9862 & 0.931 & 29.805 & 5.565 \\
\hline 07/25/00 & $16: 20: 23$ & 138.2362 & 0.931 & 29.807 & 5.565 \\
\hline $07 / 25 / 00$ & $16: 20: 38$ & 138.4862 & 0.929 & 29.807 & 5.567 \\
\hline $07 / 25 / 00$ & $16: 20: 53$ & 138.7362 & 0.929 & 29.807 & 5.567 \\
\hline $07 / 25 / 00$ & $16: 21: 08$ & 138.9862 & 0.946 & 29.807 & 5.55 \\
\hline $07 / 25 / 00$ & $16: 21: 23$ & 139.2362 & 0.956 & 29.807 & 5.54 \\
\hline $07 / 25 / 00$ & $16: 21: 38$ & 139.4862 & 0.956 & 29.807 & 5.54 \\
\hline $07 / 25 / 00$ & $16: 21: 53$ & 139.7362 & 0.966 & 29.807 & 5.53 \\
\hline $07 / 25 / 00$ & 16:22:08 & 139.9862 & 0.962 & 29.809 & 5.534 \\
\hline $07 / 25 / 00$ & $16: 22: 23$ & 140.2362 & 0.961 & 29.807 & 5.535 \\
\hline $07 / 25 / 00$ & $16: 22: 38$ & 140.4862 & 0.958 & 29.807 & 5.538 \\
\hline $07 / 25 / 00$ & $16: 22: 53$ & 140.7362 & 0.959 & 29.807 & 5.537 \\
\hline $07 / 25 / 00$ & $16: 23: 08$ & 140.9862 & 0.965 & 29.807 & 5.531 \\
\hline $07 / 25 / 00$ & $16: 23: 23$ & 141.2362 & 0.975 & 29.805 & 5.521 \\
\hline $07 / 25 / 00$ & $16: 23: 38$ & 141.4862 & 0.971 & 29.807 & 5.525 \\
\hline $07 / 25 / 00$ & $16: 23: 53$ & 141.7362 & 0.968 & 29.807 & 5.528 \\
\hline $07 / 25 / 00$ & $16: 24: 08$ & 141.9862 & 0.965 & 29.807 & 5.531 \\
\hline $07 / 25 / 00$ & $16: 24: 23$ & 142.2362 & 0.962 & 29.807 & 5.534 \\
\hline
\end{tabular}




\begin{tabular}{|c|c|c|c|c|c|}
\hline \multicolumn{5}{|c|}{ Appendix C (continued) } & \multirow[b]{2}{*}{5.53} \\
\hline 07/25/00 & $16: 24: 38$ & 142.4862 & 0.966 & 29.807 & \\
\hline 07/25/00 & $16: 24: 53$ & 142.7362 & 0.971 & 29.809 & 5.525 \\
\hline $07 / 25 / 00$ & $16: 25: 08$ & 142.9862 & 0.958 & 29.807 & 5.538 \\
\hline 07/25/00 & $16: 25: 23$ & 143.2362 & 0.922 & 29.807 & 5.574 \\
\hline 07/25/00 & $16: 25: 38$ & 143.4862 & 0.923 & 29.807 & 5.573 \\
\hline 07/25/00 & $16: 25: 53$ & 143.7362 & 0.925 & 29.807 & 5.571 \\
\hline 07/25/00 & $16: 26: 08$ & 143.9862 & 0.923 & 29.807 & 5.573 \\
\hline 07/25/00 & $16: 26: 23$ & 144.2362 & 0.919 & 29.805 & 5.577 \\
\hline 07/25/00 & $16: 26: 38$ & 144.4862 & 0.89 & 29.809 & 5.606 \\
\hline 07/25/00 & $16: 26: 53$ & 144.7362 & 0.88 & 29.809 & 5.616 \\
\hline 07/25/00 & $16: 27: 08$ & 144.9862 & 0.885 & 29.809 & 5.611 \\
\hline 07/25/00 & $16: 27: 23$ & 145.2362 & 0.886 & 29.809 & 5.61 \\
\hline 07/25/00 & $16: 27: 38$ & 145.4862 & 0.889 & 29.807 & 5.607 \\
\hline 07/25/00 & $16: 27: 53$ & 145.7362 & 0.814 & 29.807 & 5.682 \\
\hline $07 / 25 / 00$ & $16: 28: 08$ & 145.9862 & 0.83 & 29.807 & 5.666 \\
\hline 07/25/00 & $16: 28: 23$ & 146.2362 & 0.843 & 29.807 & 5.653 \\
\hline 07/25/00 & $16: 28: 38$ & 146.4862 & 0.869 & 29.807 & 5.627 \\
\hline 07/25/00 & $16: 28: 53$ & 146.7362 & 0.922 & 29.807 & 5.574 \\
\hline 07/25/00 & $16: 29: 08$ & 146.9862 & 0.919 & 29.807 & 5.577 \\
\hline 07/25/00 & $16: 29: 23$ & 147.2362 & 0.916 & 29.807 & 5.58 \\
\hline 07/25/00 & $16: 29: 38$ & 147.4862 & 0.916 & 29.807 & 5.58 \\
\hline 07/25/00 & $16: 29: 53$ & 147.7362 & 0.916 & 29.807 & 5.58 \\
\hline 07/25/00 & $16: 30: 08$ & 147.9862 & 0.913 & 29.809 & 5.583 \\
\hline 07/25/00 & $16: 30: 23$ & 148.2362 & 0.936 & 29.805 & 5.56 \\
\hline 07/25/00 & $16: 30: 38$ & 148.4862 & 1.352 & 29.807 & 5.144 \\
\hline 07/25/00 & $16: 30: 53$ & 148.7362 & 1.644 & 29.805 & 4.852 \\
\hline 07/25/00 & 16:31:08 & 148.9862 & 1.865 & 29.805 & 4.631 \\
\hline 07/25/00 & $16: 31: 23$ & 149.2362 & 1.999 & 29.817 & 4.497 \\
\hline 07/25/00 & 16:31:38 & 149.4862 & 2.121 & 29.815 & 4.375 \\
\hline 07/25/00 & $16: 31: 53$ & 149.7362 & 2.238 & 29.807 & 4.258 \\
\hline 07/25/00 & 16:32:08 & 149.9862 & 2.35 & 29.807 & 4.146 \\
\hline 07/25/00 & 16:32:23 & 150.2362 & 2.456 & 29.807 & 4.04 \\
\hline 07/25/00 & 16:32:38 & 150.4862 & 2.556 & 29.805 & 3.94 \\
\hline 07/25/00 & 16:32:53 & 150.7362 & 2.649 & 29.807 & 3.847 \\
\hline 07/25/00 & 16:33:08 & 150.9862 & 2.74 & 29.805 & 3.756 \\
\hline 07/25/00 & $16: 33: 23$ & 151.2362 & 2.825 & 29.805 & 3.671 \\
\hline 07/25/00 & $16: 33: 38$ & 151.4862 & 2.905 & 29.805 & 3.591 \\
\hline 07/25/00 & $16: 33: 53$ & 151.7362 & 2.983 & 29.805 & 3.513 \\
\hline 07/25/00 & 16:34:08 & 151.9862 & 3.058 & 29.803 & 3.438 \\
\hline 07/25/00 & $16: 34: 23$ & 152.2362 & 3.128 & 29.805 & 3.368 \\
\hline 07/25/00 & $16: 34: 38$ & 152.4862 & 3.194 & 29.805 & 3.302 \\
\hline 07/25/00 & $16: 34: 53$ & 152.7362 & 3.259 & 29.803 & 3.237 \\
\hline 07/25/00 & 16:35:08 & 152.9862 & 3.318 & 29.803 & 3.178 \\
\hline 07/25/00 & $16: 35: 23$ & 153.2362 & 3.378 & 29.803 & 3.118 \\
\hline 07/25/00 & 16:35:38 & 153.4862 & 3.434 & 29.819 & 3.062 \\
\hline 07/25/00 & $16: 35: 53$ & 153.7362 & 3.485 & 29.809 & 3.011 \\
\hline 07/25/00 & $16: 36: 08$ & 153.9862 & 3.532 & 29.805 & 2.964 \\
\hline 07/25/00 & $16: 36: 23$ & 154.2362 & 3.581 & 29.807 & 2.915 \\
\hline 07/25/00 & $16: 36: 38$ & 154.4862 & 3.626 & 29.803 & 2.87 \\
\hline 07/25/00 & 16:36:53 & 154.7362 & 3.669 & 29.803 & 2.827 \\
\hline 07/25/00 & $16: 37: 08$ & 154.9862 & 3.709 & 29.805 & 2.787 \\
\hline
\end{tabular}




\section{Appendix C (continued)}

$\begin{array}{llllll}\text { 07/25/00 } & 16: 37: 23 & 155.2362 & 3.745 & 29.801 & 2.751 \\ 07 / 25 / 00 & 16: 37: 38 & 155.4862 & 3.719 & 29.803 & 2.777 \\ 07 / 25 / 00 & 16: 37: 53 & 155.7362 & 3.765 & 29.803 & 2.731 \\ 07 / 25 / 00 & 16: 38: 08 & 155.9862 & 3.804 & 29.805 & 2.692 \\ 07 / 25 / 00 & 16: 38: 23 & 156.2362 & 3.839 & 29.803 & 2.657 \\ 07 / 25 / 00 & 16: 38: 38 & 156.4862 & 3.872 & 29.803 & 2.624 \\ 07 / 25 / 00 & 16: 38: 53 & 156.7362 & 3.901 & 29.811 & 2.595 \\ 07 / 25 / 00 & 16: 39: 08 & 156.9862 & 3.934 & 29.817 & 2.562 \\ 07 / 25 / 00 & 16: 39: 23 & 157.2362 & 3.96 & 29.807 & 2.536 \\ 07 / 25 / 00 & 16: 39: 38 & 157.4862 & 3.984 & 29.805 & 2.512 \\ 07 / 25 / 00 & 16: 39: 53 & 157.7362 & 4.01 & 29.803 & 2.486 \\ 07 / 25 / 00 & 16: 40: 08 & 157.9862 & 4.034 & 29.803 & 2.462 \\ 07 / 25 / 00 & 16: 40: 23 & 158.2362 & 4.056 & 29.803 & 2.44 \\ 07 / 25 / 00 & 16: 40: 38 & 158.4862 & 4.077 & 29.803 & 2.419 \\ 07 / 25 / 00 & 16: 40: 53 & 158.7362 & 4.096 & 29.801 & 2.4 \\ 07 / 25 / 00 & 16: 41: 08 & 158.9862 & 4.116 & 29.803 & 2.38 \\ 07 / 25 / 00 & 16: 41: 23 & 159.2362 & 4.135 & 29.803 & 2.361 \\ 07 / 25 / 00 & 16: 41: 38 & 159.4862 & 4.152 & 29.801 & 2.344 \\ 07 / 25 / 00 & 16: 41: 53 & 159.7362 & 4.168 & 29.801 & 2.328 \\ 07 / 25 / 00 & 16: 42: 08 & 159.9862 & 4.184 & 29.803 & 2.312 \\ 07 / 25 / 00 & 16: 42: 23 & 160.2362 & 4.2 & 29.803 & 2.296 \\ 07 / 25 / 00 & 16: 42: 38 & 160.4862 & 4.213 & 29.803 & 2.283 \\ 07 / 25 / 00 & 16: 42: 53 & 160.7362 & 4.227 & 29.801 & 2.269 \\ 07 / 25 / 00 & 16: 43: 08 & 160.9862 & 4.239 & 29.801 & 2.257 \\ 07 / 25 / 00 & 16: 43: 23 & 161.2362 & 4.254 & 29.801 & 2.242 \\ 07 / 25 / 00 & 16: 43: 38 & 161.4862 & 4.263 & 29.801 & 2.233 \\ 07 / 25 / 00 & 16: 43: 53 & 161.7362 & 4.273 & 29.809 & 2.223 \\ 07 / 25 / 00 & 16: 44: 08 & 161.9862 & 4.288 & 29.819 & 2.208 \\ 07 / 25 / 00 & 16: 44: 23 & 162.2362 & 4.295 & 29.805 & 2.201\end{array}$




\section{Appendix C (continued)}

This page intentionally left blank 